\title{
Experimental Facilities Division Progress Report 1997-98
}

\section{ANL/APS/TB-34 \\ Table of Contents (All links are pdf files)}

$\underline{\text { Acronyms }}$

1 INTRODUCTION

1.1 Background

1.2 Mission of the APS Experimental Facilities Division

1.3 APS User Sector Layout

1.4 XFD Organization

1.5 User Operations

1.6 APS User Administration and Technical Support

1.7 R\&D in Support of User Operations and Scientific Research

1.8 SRI-CAT

1.9 Collaborative Work

1.10 Long-Term R\&D Plans

\section{XFD OPERATIONS}

2.1 Introduction

2.2 Installation and Commissioning Status

2.3 Operations Experience

2.4 Reliability Analysis

2.5 Maintenance

2.6 Beamline Operations

2.6.1 Introduction

2.6.2 Insertion Devices

2.6.3 Front Ends

2.6.4 User Beamlines

2.6.5 User Interfaces

2.6.6 Sector 5 Front-End Problem

2.7 Interlock Systems and Instrumentation

2.7.1 The APS Personnel Safety System

2.7.2 The Equipment Protection System

2.7.3 Instrumentation

2.7.4 Controls

2.8 Experiment Floor Operations 
2.8.1 Shielding Validation of the Experiment Stations

2.8.2 Measurement of Radiation Dose Received by IDs

2.8.3 Measurement of Bremsstrahlung Absorbed Dose in Tissue Phantoms

2.8.4 Photoneutron Dose Measurements in the First Optics Enclosures

\section{USER ADMINISTRATIVE SUPPORT}

\subsection{APS Users}

3.1.1 Collaborative Access Teams

3.1.2 Independent Investigators/Collaborators

3.1.3 User Community Description

3.2 User Support

3.2.1 User Communications

3.2.2 User Registration, Orientation, and Badging

3.2.3 User Data Management

3.2.4 Support of User Advisory Groups

3.2.5 Conference and Workshop Organization and Support

3.2.6 User Agreements

3.2.7 User Accounts

3.2.8 User Policies and Procedures

3.2.9 Beamline Design Reviews

3.2.10 Technical Policy Support

3.3 User Safety

3.3.1 Experiment Safety Review

3.3.2 Independent CAT Safety Assessments

3.3.3 Safety Actions, Reviews, and Audits

\section{USER TECHNICAL SUPPORT}

4.1 X-ray Optics Fabrication and Metrology

4.1.1 X-ray Optics Metrology Laboratory

4.1.2 Deposition Laboratory

4.1.3 Fabrication Laboratory

4.1.4 X-ray Characterization Laboratory

4.1.5 X-ray Mirror Design and Characterization

4.2 Beamline Controls and Data Acquisition

4.2.1 Improvements in Scan Software

4.2.2 Improvements in Data-Storage and Display Software

4.2.3 Support for Message-Based Devices

4.2.4 Support for Remote Beamline Operation

4.2.5 Other Highlights

4.3 References

\section{R\&D IN SUPPORT OF OPERATIONS}




\section{$\underline{5.1 \text { Radiation Source Performance }}$}

5.1.1 Undulator A Performance

5.1.2 Elliptical Multipole Wiggler Performance

5.1.3 Circularly Polarized Undulator (CPU)

5.1.4 Simultaneous Operation of Two Undulators

5.1.5 5-mm Chamber and Undulator Measurements at 8.5-mm Gap

5.1.6 Storage Ring Installation - Phase-2

5.1.7 Collaborations

5.2 Beamline Engineering

5.2.1 Introduction

5.2.2 Brazing Capabilities for Beamline Components

5.2.3 Beamline and Front-End Design for New Sectors and User Support

5.2.4 Laser Doppler Angular Encoder with Sub-Nanoradian Sensitivity

$\underline{5.3 \mathrm{X} \text {-ray Optics Development }}$

5.3.1 Introduction

5.3.2 Cryogenically Cooled Silicon Monochromators

5.3.3 Diamond Monochromators

5.3.4 High-Heat-Load Optics for the Future (or Enhanced Storage Ring

Operation)

5.3.5 Other X-ray Optics Related Activities

$\underline{5.4 \text { New Instruments and Techniques }}$

5.4.1 Introduction

5.4.2 Sector 1

5.4.3 Sector 2

5.4.4 Sector 3

5.4.5 Sector 4

5.5 References

6 MAJOR PLANS FOR THE FUTURE

6.1 Summary of Major Plans for the Future

6.2 Phase-2 Initiative

6.2.1 Background

6.2.2 Beamline Plan in the APS Phase-2 Initiative

6.3 FEL Project

6.3.1 FEL Computer Code

6.3.2 The FEL Line

6.3.3 Characteristics of the FEL Undulator

6.3.4 The Optical Diagnostics

6.4 Center for Combinatorial Materials Science and Technology

6.5 Structural Genomics Project 
ANL/APS/TB-30 Table of Contents

6.6 References

7 APPENDICES

Appendix 1: 1997-1998 Publications by XFD Staff

Appendix 2: 1997-1998 Invited Presentations by XFD Staff

Appendix 3: SRI-CAT Staff and Members

Appendix 4: Review Status of APS Collaborative Access Teams

Appendix 5: User Agreement and Proprietary Account Status (8/25/98)

Appendix 6: XFD Safety Report for FY 1998 


\section{Acronyms}

\begin{tabular}{|c|c|}
\hline AFM & Atomic Force Microscope \\
\hline ALS EPU & Advanced Light Source Elliptically Polarized Undulator \\
\hline ANL & Argonne National Laboratory \\
\hline APD & Avalanche Photodiode \\
\hline APS & Advanced Photon Source \\
\hline APS RES & Advanced Photon Source - Research Initiative \\
\hline APSUO & Advanced Photon Source Users Organization \\
\hline ASD & Accelerator Systems Division \\
\hline BCM & Bent-Crystal Monochromator \\
\hline BCTF & Beamline Component Test Facility \\
\hline BESSY & Berliner Elektronenspeicherring-Gesellschaft fur Synchrotronstrahlung \\
\hline BM & Bending Magnet \\
\hline BNL & Brookhaven National Laboratory \\
\hline BPM & Beam Position Monitor \\
\hline CAT & Collaborative Access Team \\
\hline CBT & Computer-Based Training \\
\hline CCD & Charge Coupled Device \\
\hline CCST & Coordination Council for Science and Technology \\
\hline CHESS & Cornell High Energy Synchrotron Source \\
\hline $\mathrm{CM}$ & Configuration Management \\
\hline CMS & Chemical Management System \\
\hline CMB & Center for Mechanistic Biology and Biotechnology at ANL \\
\hline CPU & Circularly Polarized Undulator \\
\hline CT & Compact Tension \\
\hline CVD & Chemical Vapor Deposition \\
\hline DCM & Double-Crystal Monochromator \\
\hline DEC & Digital Equipment Corporation \\
\hline DESY & Deutsches Elektronen-Synchrotron \\
\hline DI & Deionized Water \\
\hline DMM & Double-Multilayer Monochromator \\
\hline DOE & Department of Energy \\
\hline DOT & Department of Transportation \\
\hline DX & Design Exchange \\
\hline DXRL & Deep X-ray Lithography \\
\hline EMW & Elliptical Multipole Wiggler \\
\hline EPICS & Experimental Physics and Industrial Control System \\
\hline EPS & Equipment Protection System \\
\hline EQO & ESH/QA Oversight Group \\
\hline $\mathrm{ER}$ & Environmental Research \\
\hline ESAF & Experiment Safety Approval Form \\
\hline $\mathrm{ES} \& \mathrm{H}$ & Environmental, Safety and \\
\hline (or ESH) & Health . \\
\hline ESH-TR & ESH Division's Training Section \\
\hline ESRF & European Synchrotron Radiation Facility \\
\hline ETS & Equipment Tracking System \\
\hline
\end{tabular}




\begin{tabular}{|c|c|}
\hline FDR & Final Design Report \\
\hline FE & Front End \\
\hline FEA & Finite Element Analysis \\
\hline FEEPS & Front-End Equipment Protection System \\
\hline FEL & Free-Electron Laser \\
\hline FOE & First Optics Enclosure \\
\hline FV & Fast Valve \\
\hline FWHM & Full Width Half Maximum \\
\hline GERT & General Employee Radiation Training \\
\hline HazMats & Hazardous Materials \\
\hline $\mathrm{HDF}$ & Hierarchical Data Format \\
\hline ID & Insertion Device \\
\hline II & Independent Investigator \\
\hline IIP & Individual Investigator Program \\
\hline IOC & Input/Output Controller \\
\hline IR & Infra-Red \\
\hline ISI & Interlock Systems and Instrumentation \\
\hline ISM & Integrated Safety Management \\
\hline JHQ & Job Hazard Questionnaire \\
\hline LDAE & Laser Doppler Angular Encoder \\
\hline LDDM & Laser Doppler Displacement Meter \\
\hline LDRD & Laboratory Directed Research \& Development \\
\hline $\mathrm{LN}_{2}$ & Liquid Nitrogen \\
\hline LOM & Laboratory/Office Module \\
\hline LTP & Long Trace Profiler \\
\hline LSO & Laser Safety Officer \\
\hline MCD & Magnetic Circular Dichroism \\
\hline MCS & Magnetic Compton Scattering \\
\hline MEMS & Micro-Electromechanical Systems \\
\hline MIT & Massachusetts Institute of Technology \\
\hline MSD & Materials Science Division at ANL \\
\hline OUI & Operator/User Interface \\
\hline PDR & Preliminary Design Report \\
\hline PEB & Program Evaluation Board \\
\hline PHOENIX & Phonon Excitation by Nuclear Inelastic Absorption of X-rays \\
\hline PLC & Programmable Logic Controller \\
\hline PMMA & Polymethylmethacrylate \\
\hline PS1 & Photon Shutter 1 \\
\hline PS2 & Photon Shutter 2 \\
\hline PSD & Power Spectral Density Function \\
\hline PSS & Personnel Safety System \\
\hline PZT & Piezoelectric Transducer \\
\hline QA & Quality Assurance \\
\hline
\end{tabular}




\begin{tabular}{|c|c|}
\hline $\mathrm{RBC}$ & Red Blood Cells \\
\hline $\mathrm{R} \& \mathrm{D}$ & Research \& Development \\
\hline RE & Rare Earth \\
\hline RF & Radio Frequency \\
\hline RGA & Residual Gas Analyzer \\
\hline rms & Root Mean Square \\
\hline SASE & Self-Amplified Spontaneous Emission \\
\hline SGM & Spherical Grating Monochromator \\
\hline SI & Strategic Initiative \\
\hline SPring & Super Photon Ring - 8 GeV (Japan) \\
\hline SRI & Synchrotron Radiation Instrumentation \\
\hline SXM & Scanning X-ray Microscope \\
\hline TEY & Total Electron Yield \\
\hline TLD & Thermoluminescent Dosimeter \\
\hline TMS & Training Management System \\
\hline TTL & Transitor-Transitor Logic \\
\hline TTU & Topo Test Unit \\
\hline UA2 & Undulator A (sector 8) \\
\hline UHV & Ultrahigh Vacuum \\
\hline UTIG & User Technical Interface Group \\
\hline VDOS & Vibrational Density of States \\
\hline VUV & Vacuum Ultraviolet \\
\hline XBPM & X-ray Beam Position Monitor \\
\hline XFCS & X-ray Fluorescence Correlation Spectroscopy \\
\hline XFD & Experimental Facilities Division \\
\hline $\mathrm{XFT}$ & X-ray Fluorescence Tomography \\
\hline XFM & X-ray Fluorescence Microscopy \\
\hline XIFS & X-ray Intensity Fluctuation Spectroscopy \\
\hline XMD & X-ray Microdiffraction \\
\hline XMDC & X-ray Magnetic Circular Dichroism \\
\hline XMSAS & X-ray Microbeam Small-Angle Scattering \\
\hline XPS & X-ray Polarization and Spectroscopy \\
\hline
\end{tabular}




\subsection{Background}

This Progress Report summarizes the activities of the APS Experimental Facilities Division (XFD) over the period 1997-98. The XFD personnel focused on supporting the Advanced Photon Source (APS) users from day-to-day operations support to longterm research and development (R\&D) needs. The XFD personnel would like to proudly share their major accomplishments with the readers of this report.

Over the past eighteen months, many new beamlines have begun performing scientific research, and the user presence at the APS has grown continually. At Argonne National Laboratory (ANL), the APS has become the centerpiece of user programs, and many programmatic divisions at Argonne have started to derive the benefit of this major research resource.

Thirty-five of the forty storage ring sectors include both insertion device and bending magnet sources. Of these 35 sectors, the Accelerator Systems Division (ASD) uses radiation from one undulator (35-ID) and one bending magnet source (35-BM) to perform storage ring diagnostics. The remaining 34 sectors are for the $\mathrm{R} \& \mathrm{D}$ work by the APS users.

In the first phase of the APS (Phase-1), 20 of these 34 sectors have been instrumented behind the shield wall to deliver insertion device and bending magnet radiation to the APS users. As of August 1998, the users have built beamlines in 19 of the 20 sectors as planned, and XFD personnel have installed and commissioned insertion devices and beamline front ends to provide radiation to all the APS user beamlines. In addition, Personnel Safety Systems (PSS) have been designed to meet user needs. These PSS have been installed, validated, commissioned, and operated in over 80 experiment stations on these beamlines by XFD personnel in time to meet users' objectives. The majority of these beamlines have taken advantage of the 'standard and modular' beamline components designed, constructed, and tested by XFD.

The continued R\&D support, advice, and guidance provided to the APS Collaborative Access Teams (CATs) by the XFD personnel has contributed to realizing early research at the majority of user beamlines. The Synchrotron Radiation Instrumentation (SRI) CAT, made up primarily of XFD personnel, has continued to make a major impact on the development of new instruments and techniques, as well as on new areas of science, using beamlines in three sectors (1, 2, and 3). The 'Scientific Members' of SRI-CAT have participated extensively with the XFD staff in performing frontier scientific research.

SRI-CAT has begun construction of a beamline in sector 4 that will be dedicated to the development of instruments and techniques to explore the frontiers of science using polarized X-rays. In addition, XFD is now getting ready for newer CATs planning to build beamlines in sectors beyond 21 .

This report summarizes many of the primary activities and accomplishments of the XFD personnel in supporting APS users. 


\subsection{Mission of the APS Experimental Facilities Division}

The mission of the XFD is unchanged and is consistent with the vision of the APS to function as a reliable and preeminent source of synchrotron radiation for APS users.

XFD believes that we can best serve the APS user community by investing in three important goals: reliable and successful operation, high-quality user technical and administrative support, and innovative R\&D in support of user operations and scientific research. These goals enable us to go beyond the traditional role of Department of Energy (DOE) user facilities to create an intelligent partnership with our users.

We commit ourselves to an organization that shares the following principles:

- Understanding our users' operational goals and striving to exceed their needs

- Providing seamless support to our users in all areas

- Creating a rewarding, enriching, and collaborative $R \& D$ environment for our staff and the users to facilitate the long-term success of the APS as the premier user facility in the world

- Expanding our worldwide leadership role in the synchrotron radiation community
- Assuring the safety of APS users, visitors, and APS/XFD personnel, and the protection of the environment

- Approaching our daily work with enthusiasm, a dedication to users and a sense of humor

\subsection{APS User Sector Layout}

In Figure 1.1, the most current layout of the APS experiment hall floor and allocation of sectors to various CATs is shown. Each sector consists of two sources for beamlines. In all, 18 sectors have undulators, one sector (BioCARS) has a wiggler, and the last (BESSRC) has an elliptical multipole wiggler for the production of circularly polarized radiation. Except for the newly formed UNI-CAT-2 (sector 34), the beamlines constructed in all other sectors have received radiation. The two new sectors recently assigned are sector 4 to SRICAT and sector 32 to COM-CAT, in which beamline construction is now beginning. The major scientific disciplines of each of the sectors are identified in Figure 1.1. They are (a) condensed matter physics, chemical science, and materials science, (b) health science (biology), (c) environmental science, geoscience, soil science, and agricultural science, and (d) synchrotron radiation instrumentation and techniques. It is in this last area that XFD has a major commitment. 


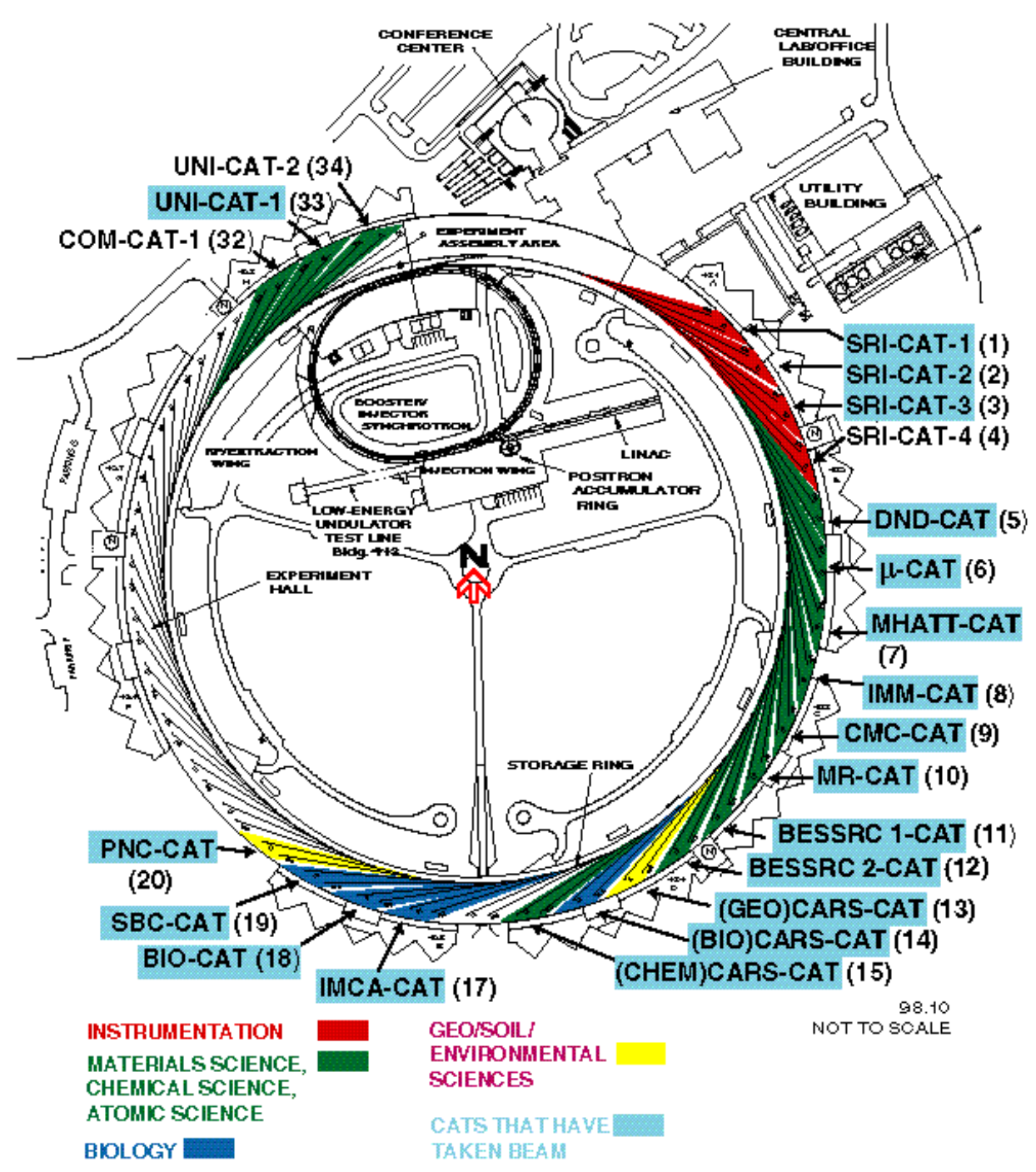

Fig. 1.1 APS Collaborative Access Teams by sector and discipline.

\subsection{XFD Organization}

The XFD organization has three functional areas as shown in Fig 1.2. The XFD organization structure, shown in Fig 1.3, defines various groups by specialization. This structure folds into functional organization and guarantees excellent communication and interaction across the boundaries of the groups to meet both the groups' and XFD's objectives.

\subsection{User Operations}

This functional unit supports user operations at the APS and consists of the Beamline Operations Group, the Safety Interlocks and Instrumentation Group, and, recently, the 


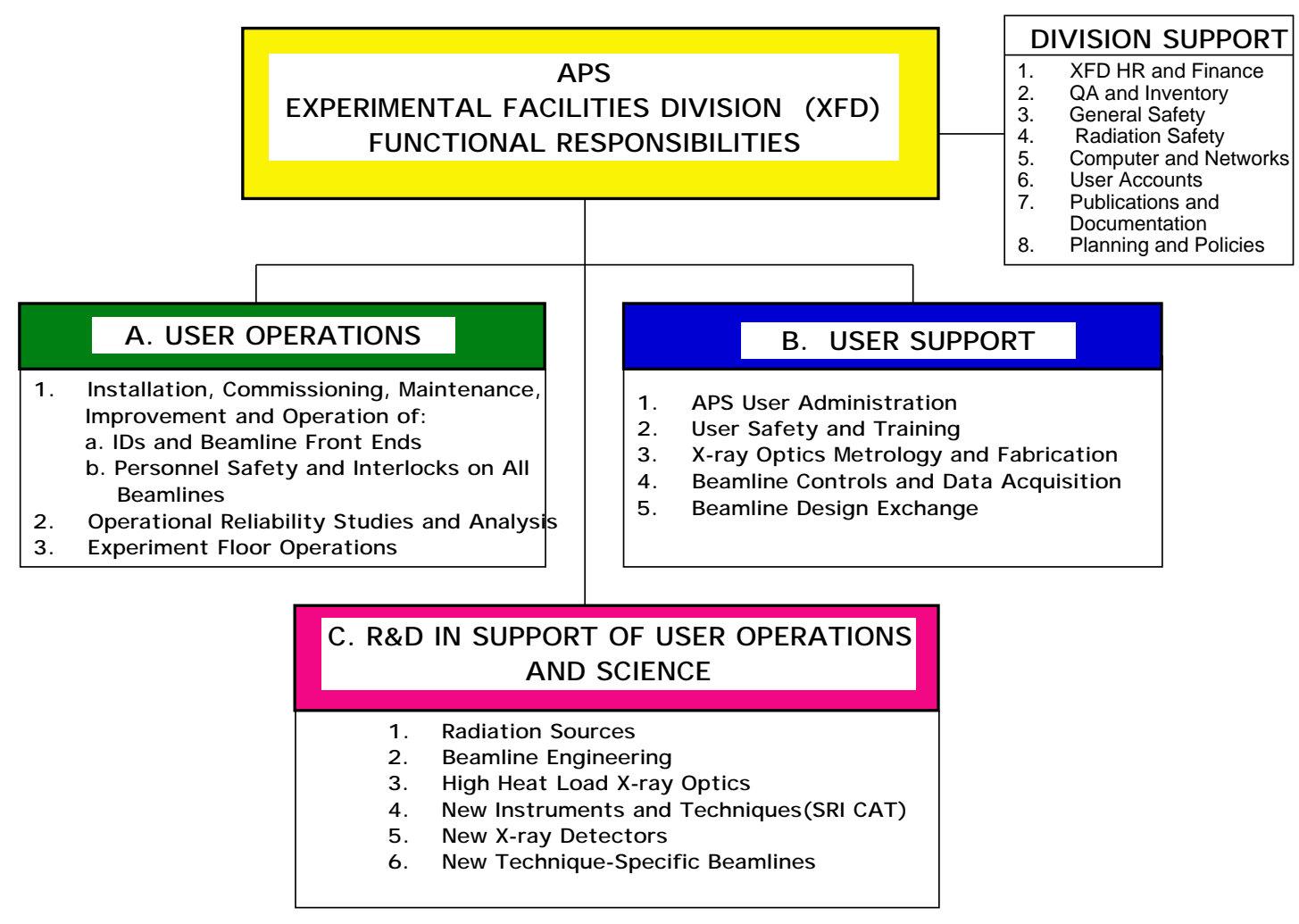

Fig. 1.2 XFD is organized into three functional areas: (A) User Operations, (B) User Support, and (C) R\&D in Support of User Operations and Science.

Experiment Floor Operations Group. The groups use the expertise of the XFD radiation physicist and a project engineer in all user beamline design, procurement, and commissioning activities. During the past 18 months, these groups have performed installation, commissioning, and routine maintenance of all insertion devices, beamline front ends, experiment enclosures, PSS, and Equipment Protection Systems (EPS), in addition to reliability studies and analysis leading to design changes and upgrades of components. Their main objective is to assure the highest level of reliability of operations of the radiation sources, beamline components, and PSS. There has been less than $0.7 \%$ of downtime in user beam time over a 12 month period (April 97 - March 1998) associated with
XFD operations. This has supported the highest level of productivity from the APS users. Continued operational improvements are planned to decrease the downtime even further.

The number of experiment stations has nearly tripled during the past 18 months. The XFD support to the CATs on these experiment enclosures starts with their design, management of procurement contracts, installation supervision (both technical and safety) of the contractor workers, installation of the PSS, commissioning, shielding verification (and revalidation) using Argonne health physics technicians, and finally operations. At the present time over 80 experiment stations are operational at the APS. 


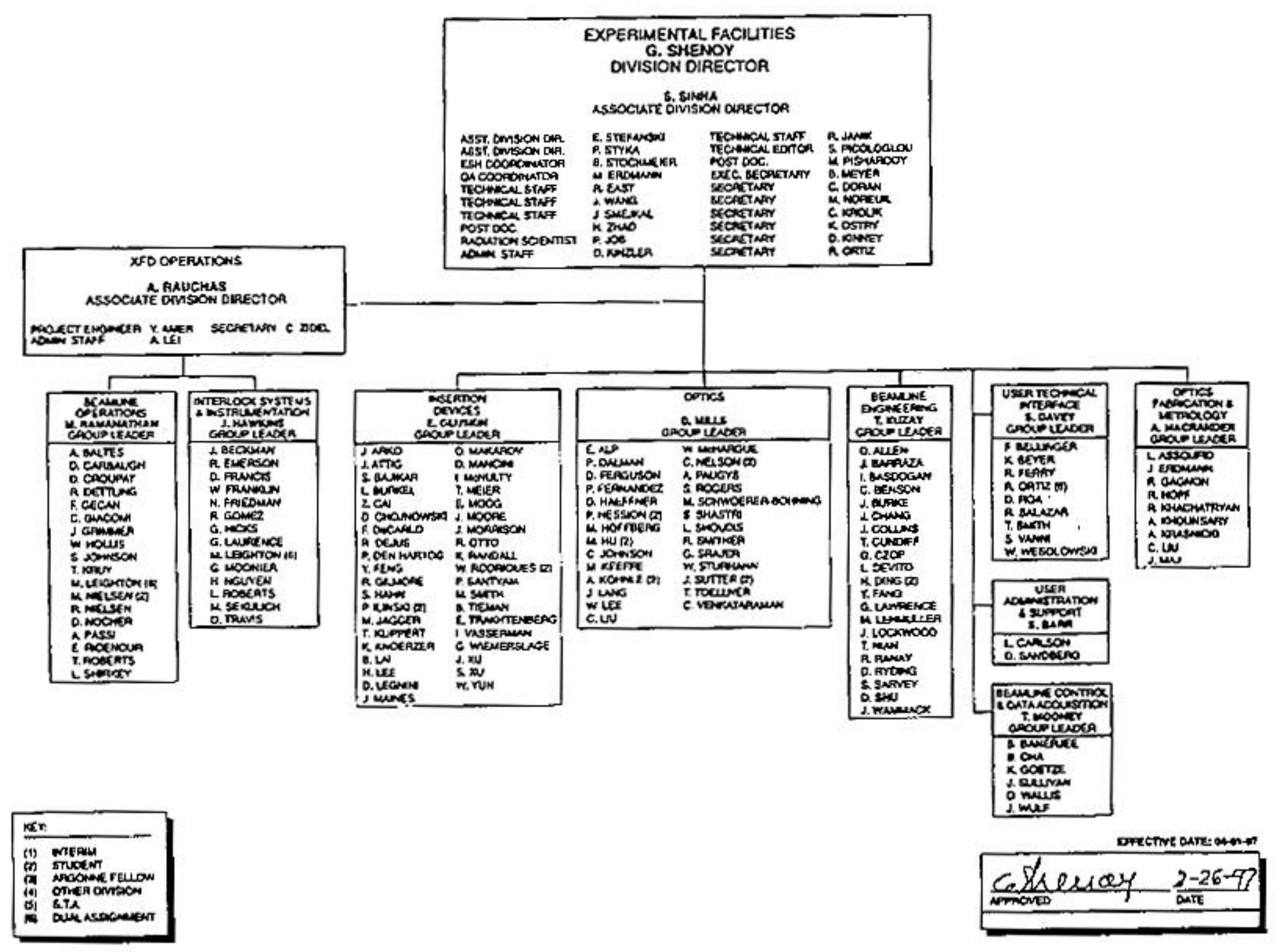

Fig. 1.3 Experimental Facilities Division oryunization chart 
In terms of reorganization, the Floor Operations Group consisting of floor coordinators was formed during this year and reports to the Associate Division Director for XFD Operations. The Floor Operations Group meets day-to-day user needs, provides oversight on user safety, and maintains the authority to suspend any of the user activities if it is felt that unsafe conditions may exist. The floor coordinators are the first point of contact on all topics of interest to the APS users on the experiment hall floor.

\subsection{APS User Administration and Technical Support}

This activity is supported by many groups: User Administration and Support, User Technical Interface, X-ray Optics Metrology and Fabrication, Beamline Controls and Data Acquisition, and the staff involved in the operation of the Design Exchange. These groups continue to provide support to the APS users in all areas in a seamless fashion. Some of their principal activities include:

- Technical support in the design of instruments, beamlines and experiments

- Administrative support, user orientation, safety training, etc.

- Development of user policies and procedures

- Development and maintenance of user databases to support user needs, user access to the APS, user demographics, safety training records, experiment safety approval records, publication records, scientific program review records, independent investigator proposal activities, etc.

- Beamline controls and data acquisition support to most of the APS CATs, and support of the capability for remote operation of experiments at the APS beamlines from user home institutions

- Design and fabrication of a variety of crystal, mirror, and multilayer optics for APS CAT beamlines and evaluation of their performance

- Management of cost accounts for over 100 APS user institutions with dollar amounts ranging from a few thousand to many millions including the costs of stock-room items as well as major beamline components

\subsection{R\&D in Support of User Operations and Scientific Research}

$R \& D$ in support of user operations is an important function primarily provided by the Insertion Device Group, Beamline Engineering Group, and X-ray Optics Group. These groups support the XFD Operations Groups in meeting their objectives by providing expert guidance and redesign assistance as needed. This arrangement has worked well during the past year.

A second responsibility is to perform $R \& D$ to address unique issues for the present and the future needs of the users, such as 
superior beam stability, mechanical and optical components for 300-mA operation, support of user experiments during 'top-up' mode, special operating modes to meet the needs of all users, and unique radiation sources and beamline requirements.

A third functional responsibility is to provide the highest quality leadership in synchrotron radiation instrumentation and technique development in order to support and enhance the APS users' scientific research. Many of these activities, having both short- and long-term scientific benefit to the user community, are performed through SRI-CAT.

The accomplishments of these groups over the past 18 months have been extensive as measured from the quality and number of publications and invited presentations. (See Appendix 1 for publications and Appendix 2 for invited presentations.)

\subsection{SRI-CAT}

New directions in science result from scientific revolution. In the past, scientific revolutions-big or small, global or local-have been driven by either new concepts or new tools. The staff involved in SRI-CAT firmly believe that tool-driven revolution adds much to the progress of science. In his book Imagined Worlds, the author Freeman Dyson ${ }^{1}$ points out that the effect of a concept-driven revolution is to explain old things in new ways. The effect

1 Freeman Dyson, Imagined Worlds, Harvard University Press, Cambridge, Massachusetts, 1997. of a tool-driven revolution is to discover new things that have to be explained. During the past year, SRI-CAT has been involved in a host of tool-driven discoveries in synchrotron-based science. Examples include microprobe tools leading to quantitative studies in agricultural and environmental sciences, understanding of diagnostics and treatment of cancer, the role of residual stress in relationship to failure in materials, and the ability to measure element-specific dynamics of atoms in complex fluids; high-energy-resolution tools leading to the science of phonons in thin films and amorphous systems; $\mathrm{x}$-ray polarization tools leading to tomographic images of magnetic domains and understanding of magnetic surface roughness so very important in modern data storage technologies; deep $\mathrm{x}$-ray lithography, which would lead to the development of new microscopic tools for physical measurements; high-energy x-ray scattering to probe glasses and liquids; soft and hard $\mathrm{x}$-ray tomographic imaging tools to explore their application to modern technology, such as integrated circuits, and to ancient artifacts, such as dinosaur teeth; refining the broad spectrum of absorption and scattering tools to open the new field of $\mathrm{x}$-ray archaeometallurgy; inelastic and Raman x-ray scattering tools to understand collective phonon and electron behavior of unique condensed systems; coherence-based tools in the soft and hard $\mathrm{x}$-ray energies to provide the new capability to study fluctuations in condensed matter; and the list goes on.

These tool-driven activities will remain the main focus of SRI-CAT for years to come and will complement the traditional responsibility of supporting the user community by providing basic resources in 
the development of new optics, beamline components, new techniques, etc.

The accomplishments and future plans of SRI-CAT are discussed in more detail elsewhere in this document. (See Appendix 3 for a list of current SRI-CAT members.)

\subsection{Collaborative Work}

During the past year the major collaborative activities of the XFD staff have been:

- Design and construction of all undulator vacuum chambers for the new BESSY II storage ring in Berlin

- Design and construction of the vacuum chamber for the freeelectron laser (FEL) project TESLA at HASYLAB

- Performance evaluation of the TESLA FEL undulator system

- Construction of critical high-heatload components for the SPring-8 undulator beamline front ends

- Design support to the beamline group at the synchrotron radiation facility in Taiwan

- Design and delivery of an 8-mmaperture undulator vacuum chamber to the European Synchrotron Radiation Facility (ESRF)

- Beamline design support to COM$\mathrm{CAT}$, and assistance in managing
State of Illinois funds for the beamlines

- Tuning of an undulator to meet unique technical specifications for the operation of a 5-micron selfamplified spontaneous emission (SASE) FEL at Brookhaven National Laboratory (BNL)

This work is in addition to innumerable hours of technical support and advice provided to all APS users and the CATs. These efforts point to the high regard of the world-wide synchrotron radiation community for work performed by XFD staff.

\subsection{Long-Term R\&D Plans}

The staff in XFD actively participates in long-term R\&D activities. These activities are supported by Laboratory Directed Research and Development (LDRD) funds distributed by the Argonne National Laboratory Director through a laboratorywide competitive process. These funds are provided for the following four categories of initiatives:

1. Strategic Initiatives of the Laboratory (SI)

2. Research Initiatives of the APS (APS-RES)

3. Programs approved by the Coordination Council for Science and Technology to encourage interactions between various Argonne Divisions (CCST) 
4. Individual Investigator program to promote unique ideas from individuals (IIP)

The following is a list of LDRD programs from the XFD staff funded during FY 1998:

1. Development of a long undulator line for a new generation of synchrotron radiation sources (SI)

2. Radiation damage to Nd-Fe-B permanent magnets due to very high radiation doses $(\mathrm{SI})$

3. Investigation of a SASE process in a 5-micron FEL (SI)

4. Development of X-ray intensity fluctuation spectroscopy (XIFS) for study of atomic-scale equilibrium dynamics (SI with the Materials Science Division - MSD)

5. Anomalous inelastic x-ray scattering with meV resolution (SI)
6. Chemical vapor deposition (CVD) diamond imaging detector (SI)

7. Nanometer-resolution x-ray zone plates (SI)

8. High-speed shutter for temporal modification of the APS x-ray beam (SI)

9. Development of micromachining technique based on deep x-ray lithography (DXRL) (APS-RES)

10. Low work function coatings and LIGA-type fabrication (CCST with MSD)

11. Short-focal-length crystal lens for use in medical imaging (IIP)

12. Compact laser Doppler linear encoder with near-angstrom resolution (IIP) 


\subsection{Introduction}

The XFD Operations Organization is responsible for assuring that the APS effectively meets the operational needs of the user community and for assuring that XFD and user activities conform to the applicable requirements. In support of the APS user activities, the XFD Operations Organization also gathers specific facility operating requirements, integrates the requests, determines the operating modes that are needed to meet the requirements, and works with the Accelerator Systems Division (ASD) to satisfy the requirements. The Operations Organization is under the direction of an Associate Division Director for Operations and includes three major groups: the Beamline Operations Group, the Interlock Systems and Instrumentation Group, and the Experiment Floor Operations Group, as well as support staff to aid the group activities. The latter group was organized recently and consists of the floor coordinators, who were transferred to Operations from the User Technical Interface Group. The activities of these groups are described in more detail in the following sections.

\subsection{Installation and Commissioning Status}

The APS storage ring design incorporates a magnetic lattice with 35 5-meter-long straight sections available for installation of insertion devices (IDs). The design also incorporates the necessary beam ports for extracting radiation from 35 of the 80 storage ring bending magnets (BMs). With each sector containing an ID beamline and a BM beamline, the APS can accommodate a total of 70 beamlines. The funding for the
APS Project included the funds to construct 20 sectors worth of front ends (FEs) and IDs available for user research and an additional sector for particle beam diagnostics studies by the APS facility. All of these FEs and IDs have been installed, and most have been commissioned and are providing beam to the user beamlines. Two additional sectors have received funding, and work is underway to fabricate the necessary IDs and FE components. Installation of these components is planned to begin in 1999. The remaining 12 IDs and 24 FEs will be built and installed as future funding becomes available.

User beamline installation continues, although the installation schedule is primarily governed by the user funding availability. The APS personnel are responsible for managing the installation contracts of the experiment stations and beamline utilities. As of July 1998, 101 experiment stations have been completed on 31 beamlines. Of these 31 beamlines, all have had x-rays delivered to at least the first optics enclosure (FOE). The dates for the start of commissioning, for each of these beamlines, are shown in Table 2.1. Another seven stations are either under construction or are planned for the near future. The current beamline status is shown in Fig. 2.1.

\subsection{Operations Experience}

It is now three and one-half years since the storage ring began commissioning on February 20, 1995. The facility has developed and improved substantially over that period. In calendar year 1996, about 2000 hours of time were scheduled for user operations. Last year, this number was 
Table 2.1. Dates of First Commissioning of APS Beamlines

\begin{tabular}{|c|c|}
\hline Beamline & Date of First Beam \\
\hline 1-BM & $3 / 26 / 95$ \\
\hline 1-ID & $8 / 9 / 95$ \\
\hline 2-BM & $6 / 24 / 96$ \\
\hline 2-ID & $3 / 26 / 96$ \\
\hline $3-I D$ & $1 / 24 / 96$ \\
\hline 5-BM & $3 / 27 / 96$ \\
\hline 5-ID & $5 / 2296$ \\
\hline 6-ID & 2/3/98 \\
\hline 7-BM & $11 / 11 / 97$ \\
\hline $7-I D$ & $8 / 16 / 96$ \\
\hline 8-ID & $8 / 17 / 96$ \\
\hline 9-BM & $3 / 31 / 98$ \\
\hline 9-ID & $3 / 31 / 98$ \\
\hline 10-ID & $8 / 8 / 96$ \\
\hline 11-ID & $1 / 14 / 97$ \\
\hline 12-BM & $3 / 26 / 96$ \\
\hline 12-ID & $5 / 20 / 96$ \\
\hline 13-BM & $9 / 17 / 96$ \\
\hline 13-ID & $9 / 27 / 96$ \\
\hline 14-BM & $4 / 21 / 97$ \\
\hline 14-ID & $4 / 22 / 97$ \\
\hline 15-ID & $6 / 16 / 98$ \\
\hline 17-BM & $10 / 14 / 96$ \\
\hline 17-ID & $7 / 5 / 96$ \\
\hline 18-ID & $6 / 12 / 97$ \\
\hline 19-BM & $625 / 96$ \\
\hline 19-ID & $3 / 26 / 96$ \\
\hline 20-BM & $5 / 26 / 98$ \\
\hline 20-ID & $12 / 18 / 96$ \\
\hline 33-BM & $6 / 30 / 98$ \\
\hline 33-ID & 7/3/96 \\
\hline 35-ID & $3 / 6 / 97$ \\
\hline
\end{tabular}

3500 hours. This year, the facility will provide 4500 hours of user time, and the plan is to provide 5000 hours in 1999. Troublesome systems have been identified and major faults corrected. This has led to a significant increase in the availability of the beam and has also led to a significant decrease in the number of faults that caused the stored beam to be dumped. The improvements have involved significant effort from personnel of both ASD and XFD. Reliability issues relative to XFD components are discussed in the next section. Unplanned beam dumps not only decrease the available beam time due to the subsequent refill but also cause additional loss of time as optics and other experimental equipment recover from the thermal cycle. This effect varies from beamline to beamline and cannot be accounted for in the operating statistics. The user run statistics from the start of detailed record keeping in June 1996 to the present are shown in Table 2.2.

Since the facility has little control over the installation of user beamline components, the schedule for user beamline commissioning and operation is governed by each user organization. However, XFD Operations monitors the amount of beam usage by tracking the amount of time that the FE shutters are open. This parameter has increased significantly over the last 18 months and is shown in Fig. 2.2 as the sum of shutter open hours per available hour of user time. This number substantiates the ever-increasing number of experiment safety reviews that are being submitted by the user groups.

The operating schedule continues to be optimized as storage ring, ID, FE and user needs are better defined. As the understanding of storage ring performance increases, the time allocated to accelerator studies has decreased from $30 \%$ to $15 \%$ of the operating time. Since the current installation work for the FEs has been completed, longer shutdowns are not scheduled as frequently, and user runs are considerably longer, lasting as long as 8-9 weeks. However, to facilitate repairs during long user runs, a total of seven shifts are set 


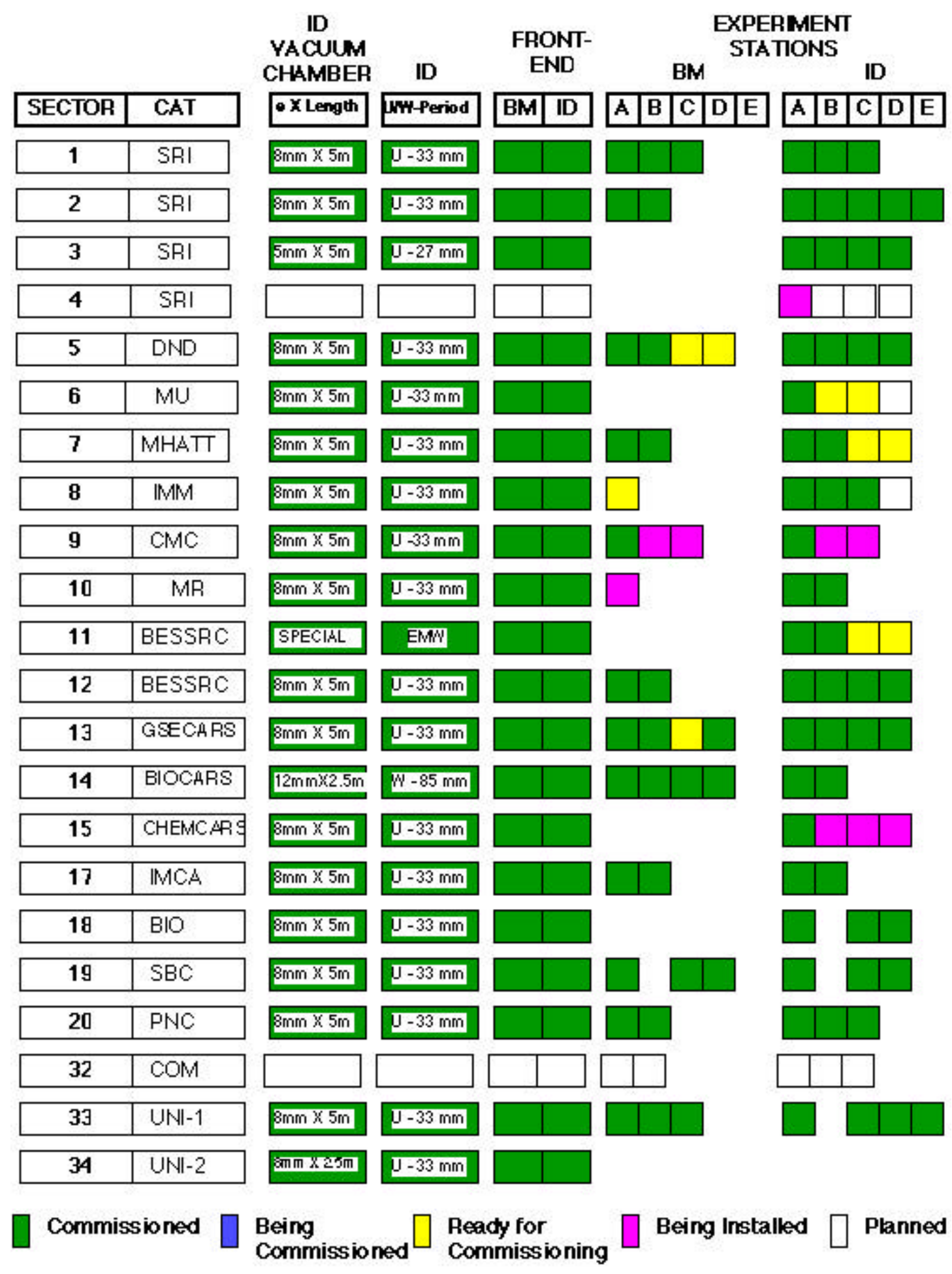

Fig. 2.1 APS CAT Beamline Status as of July 1998. 
Table 2.2. User run statistics

\begin{tabular}{|c|c|c|c|c|c|}
\hline User Run & $\begin{array}{l}\text { Run start / } \\
\text { end dates }\end{array}$ & Scheduled hours & Available hours & Availability \% & Faults per day \\
\hline $96-4$ & $\begin{array}{c}6 / 22 / 96- \\
7 / 7 / 96\end{array}$ & 300.0 & 239.8 & $79.9 \%$ & NA \\
\hline $96-5$ & $\begin{array}{l}8 / 5 / 96- \\
8 / 19 / 96\end{array}$ & 324.0 & 201.9 & $62.3 \%$ & 8.3 \\
\hline $96-6$ & $\begin{array}{l}9 / 17 / 96- \\
10 / 20 / 96\end{array}$ & 596.0 & 456.0 & $76.5 \%$ & 7.4 \\
\hline $96-7$ & $\begin{array}{c}12 / 10 / 96- \\
12 / 23 / 96\end{array}$ & 268.0 & 183.0 & $68.3 \%$ & 3.4 \\
\hline 1996 & & 1488.0 & 1080.7 & $72.6 \%$ & \\
\hline 97-1 & $\begin{array}{l}1 / 7 / 97- \\
1 / 25 / 97\end{array}$ & 376.0 & 264.8 & $70.4 \%$ & 5.5 \\
\hline $97-2$ & $\begin{array}{c}2 / 25 / 97- \\
3 / 9 / 97\end{array}$ & 296.0 & 206.0 & $69.6 \%$ & 5.7 \\
\hline $97-3$ & $\begin{array}{c}4 / 8 / 97- \\
5 / 3 / 97\end{array}$ & 480.0 & 290.0 & $60.4 \%$ & 6.8 \\
\hline $97-4$ & $\begin{array}{c}5 / 28 / 97- \\
6 / 15 / 97\end{array}$ & 384.0 & 327.3 & $85.2 \%$ & 1.9 \\
\hline $97-5$ & $\begin{array}{c}7 / 15 / 97- \\
8 / 3 / 97\end{array}$ & 408.0 & 318.5 & $78.1 \%$ & 4.4 \\
\hline $97-6$ & $\begin{array}{c}8 / 19 / 97- \\
9 / 29 / 97\end{array}$ & 864.0 & 752.9 & $87.1 \%$ & 1.4 \\
\hline $97-7$ & $\begin{array}{c}10 / 20 / 97- \\
11 / 24 / 97\end{array}$ & 744.0 & 699.4 & $94.0 \%$ & 0.58 \\
\hline 1997 & & 3552.0 & 2858.9 & $80.5 \%$ & \\
\hline 98-1 & $\begin{array}{l}1 / 12 / 98- \\
2 / 16 / 98\end{array}$ & 712.0 & 655.4 & $92.0 \%$ & 0.79 \\
\hline $98-2$ & $\begin{array}{l}3 / 9 / 98- \\
4 / 12 / 98\end{array}$ & 736.0 & 655.7 & $89.1 \%$ & 0.99 \\
\hline $98-3$ & $\begin{array}{c}5 / 18 / 98- \\
7 / 12 / 98\end{array}$ & 1168.0 & 1100.2 & $94.2 \%$ & 0.62 \\
\hline 1998 & & 2616.0 & 2411.2 & $92.2 \%$ & \\
\hline
\end{tabular}




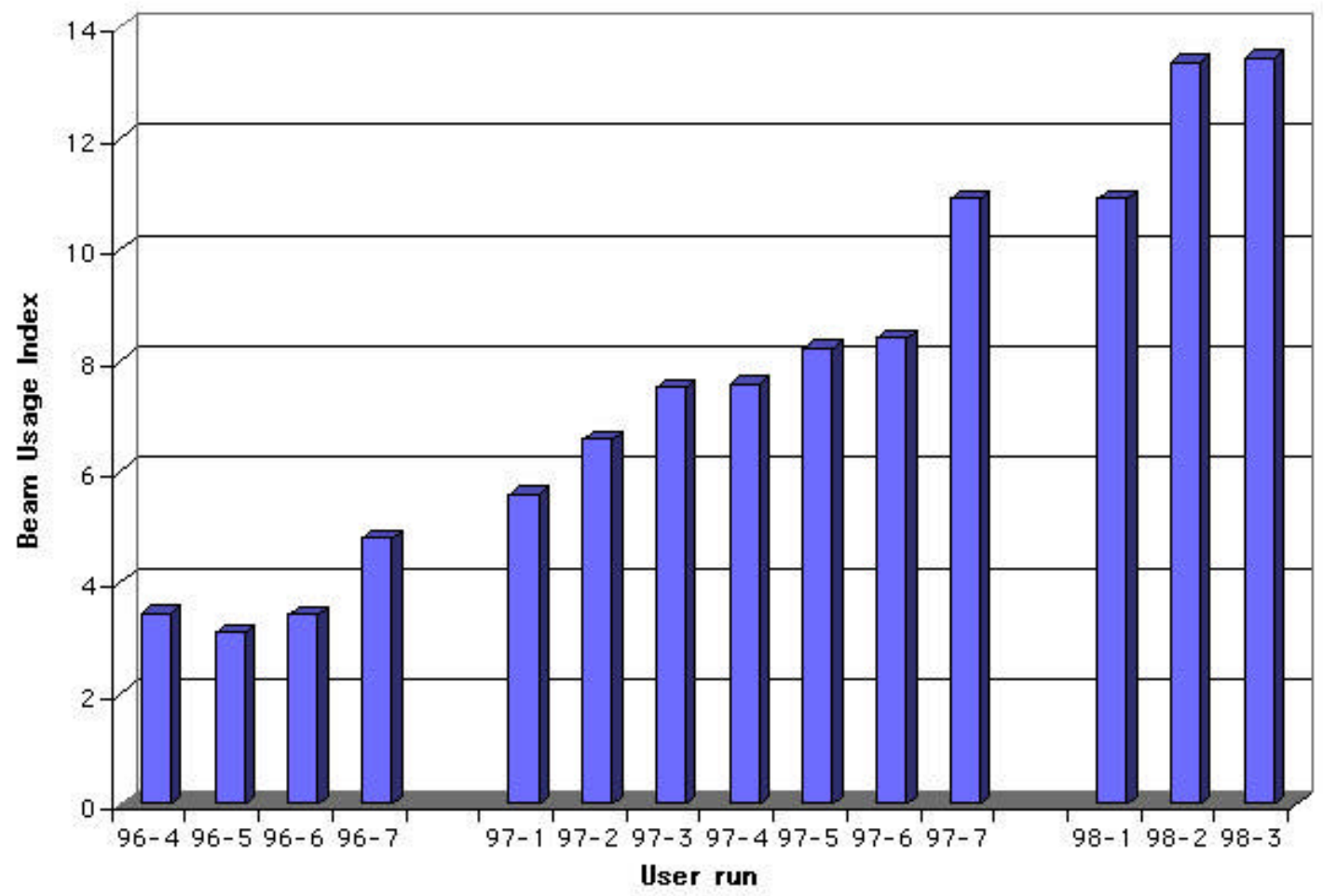

Fig. 2.2 Beamline shutter use plotted as as the sum of shutter open hours per available hours of user time.

aside during each two-week period for maintenance, repair and/or accelerator studies: six continuous shifts every two weeks and a single shift during the intervening week. The available "free" time will decrease even further next year as 5000 hours are provided for users. Since two additional FEs are also scheduled for installation next year, precise scheduling will be required to accomplish the installation tasks within the allotted times without impacting the user runs.

\subsection{Reliability Analysis}

Statistical data gathering for the Advanced Photon Source beamlines is an on-going effort aimed at monitoring the reliability of the operational systems (IDs and FEs) down to the components level. This effort is targeted at achieving the following goals:

- Increase availability of beam time to the user community

- Minimize failures and prevent their recurrence

- Predict failures before occurrence

Failures or malfunctions of any equipment that is required to directly support or operate the beamlines or that is related to personnel or equipment safety must be reported and tracked to resolution. Failures are diagnosed and followed up by the cognizant 
individuals and tracked/analyzed by the quality assurance (QA) reliability engineers.

All system/component performance data from the Experimental Physics and Industrial Control System (EPICS) is constantly logged and monitored. This facilitates data gathering and analysis of specific trends and provides the flexibility for advance warning on failures. Problems can then be dealt with in a pro-active manner.

The life history of each critical component is maintained in the Equipment Tracking System (ETS). This is a database system written in ORACLE designed to archive key information on beamline critical components.

The ETS can keep a complete history of each individual component from incoming inspection to failure and/or removal from service. In addition, users can be notified of maintenance and calibration requirements of components when applicable. Hard copy reports are also available of all data to make analysis much easier and more useful.

This database has been adapted for use on the APS Web page. The main benefit of this exercise is to make the data more accessible for users. On the Web, a user/operator is able to obtain a complete FE or ID component list on-screen simply by clicking on the appropriate sector prompt. The user could then click on a specific component in the list, and a pop-up window will appear with more detailed information on the component of interest.

Another benefit of adapting the ETS to the Web is the ability to connect the ETS database fields with current XFD Operations
Web sites. XFD Operations is currently employing a failure reporting system called Trouble Reports on the Web. Trouble Reports are generated when any problem arises during normal operations. Data gathered from the trouble reports and the repair $\operatorname{logs}$ are analyzed for failure correlations and trends.

In order to pro-actively maintain the beamline components, the ETS also has the capability of generating maintenance schedules for performing preventive maintenance during beam shutdown periods.

To date, 43 FEs and 21 ID systems are in the operational stage. Failure or malfunction data have been collected on these systems by XFD personnel through the construction, installation, and operation phases of the APS FEs and IDs. The data have been organized into two main groups: 1) critical component rejections during incoming inspection, and 2) failures after component installation and operation. Rejections during incoming inspection are documented using the ANL nonconformance reports and are also entered into the XFD ETS database. Component anomalies after installation and operation are logged into the XFD Operations Trouble Reports, which are Web based.

Operational data on the APS beamlines gathered to date have been analyzed. The number of failures documented thus far are shown in Fig 2.3. For the operational analysis, only components that failed and were either removed from service or repaired and reinstalled were included. Figure 2.3 shows both the number of rejections at incoming inspection to date as well as the failures during operations for each quarter starting with the second quarter of 1996. Rejections at incoming inspection 


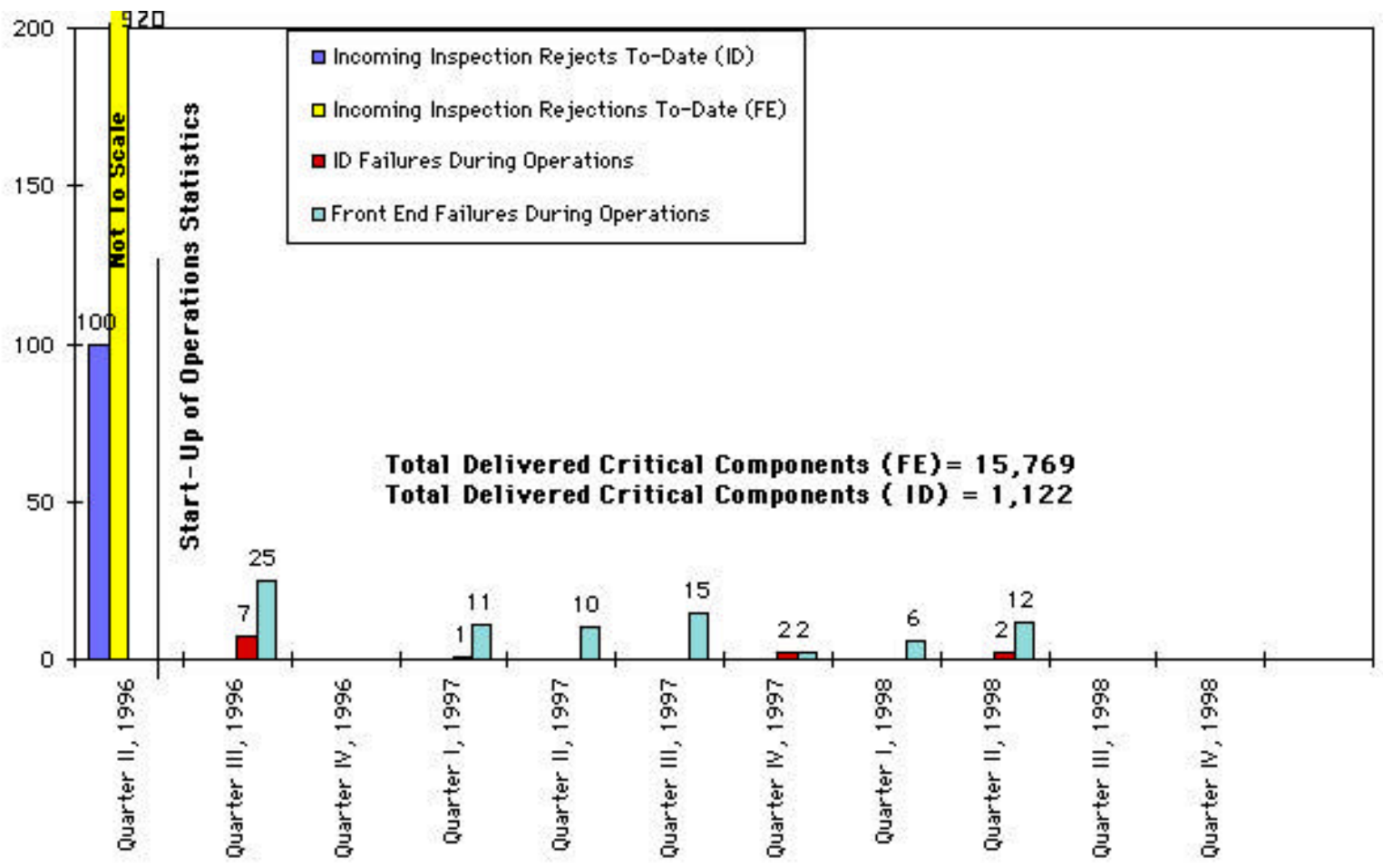

Fig. 2.3 Statistical data on component performance.

do not appear after Quarter II 1996, because the majority of critical components for the FEs and IDs were already inspected at that time. A manufacturing problem with the FE pressure transmitters led to rejection of 380 units accounting for $41 \%$ of the total rejections.

Failures after installation and operation fall into three main categories: mechanical, electrical, and vacuum. Electrical and mechanical failures are the most prevalent, occurring in cases such as modules for the PSS and EPS, encoders, controllers, and pressure transmitters. To a lesser extent, vacuum leaks have been recognized on $\mathrm{FE}$ equipment as well.

Analysis of the statistical data gathered for the period covered by this report indicates the following:
1. The XFD Operations contribution to $\mathrm{x}$-ray beam downtime during the 12month operational cycles (from Quarter II 1997 to Quarter II 1998) is depicted in Fig. 2.4. With a total of 5496 hours of user beam time, the XFD operations systems contributed to 37.4 hours of downtime representing $0.68 \%$ of the total scheduled user beam time.

2. A graphical representation of the systems contributing to the XFD operational downtime and the distribution is shown in Fig. 2.5. Further breakdown of the root cause for failures and trends occurring in each system indicates the following: 


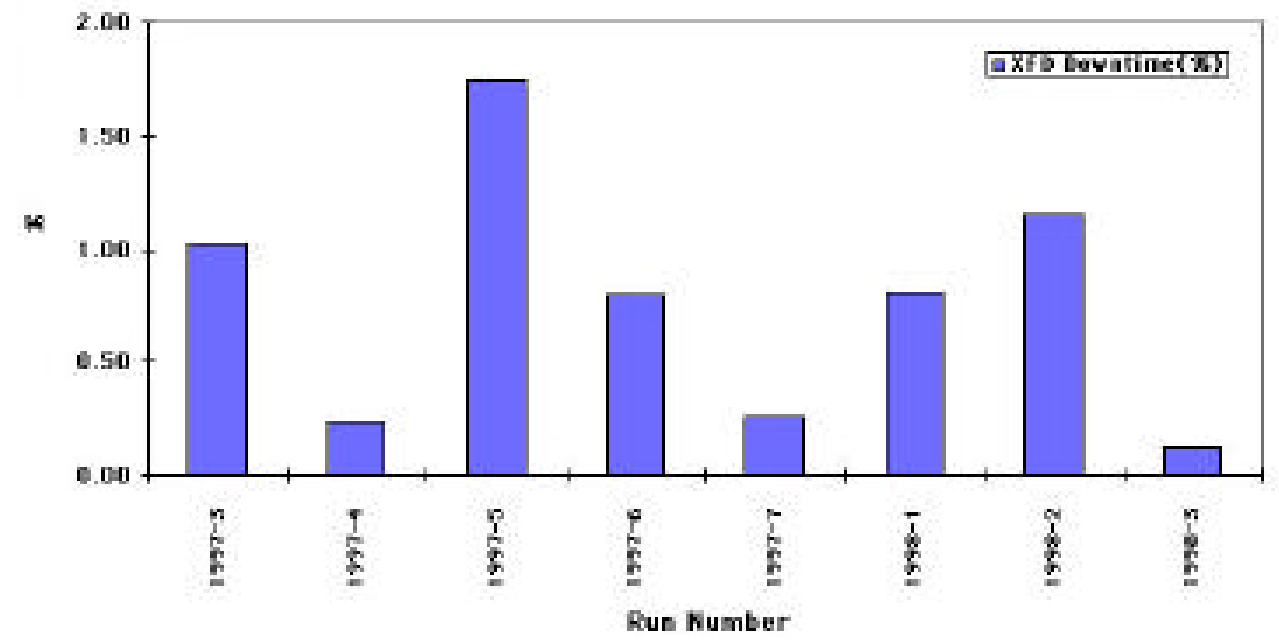

Fig. 2.4 Percentage of XFD downtime compared to scheduled beam running time.

- The PSS system failures can be broken down into two main categories: 1) photon shutters and 2) enclosure doors. Over $80 \%$ of the FE shutter failures involved only the second photon shutter (PS2). The one signature symptom of the shutter problem is a slow opening actuator. An ongoing effort is in place to identify the root cause of the problem. Corrective action will be implemented based on these results. Most station-door-related PSS failures were sluggish door operation, usually due to pneumatics problems. A program is now in place to provide semiannual preventive maintenance to all beamline enclosure doors.

- Half of the ID system failures reported were for motor-related problems, primarily for stalling of IDs prior to upgrading the stepper motor drivers from 8- to 12-amp units. Another quarter of the failures were due to ID control hardware problems. Reset errors from the linear encoders contributed to this. Encoders that exhibited this problem have been readjusted and or modified to prevent recurrence. The remaining problems related to the ID system were for access security/gateway and software problems, which are nonrecurring in both of these areas.

- Almost half of FE system failures reported during the period covered by this report are related to the cooling water system, with transmitter problems being the major cause. Vacuum-related problems were the second source of failure for the FE system, either from actual vacuum leaks or from very high beam-induced outgassing, which 


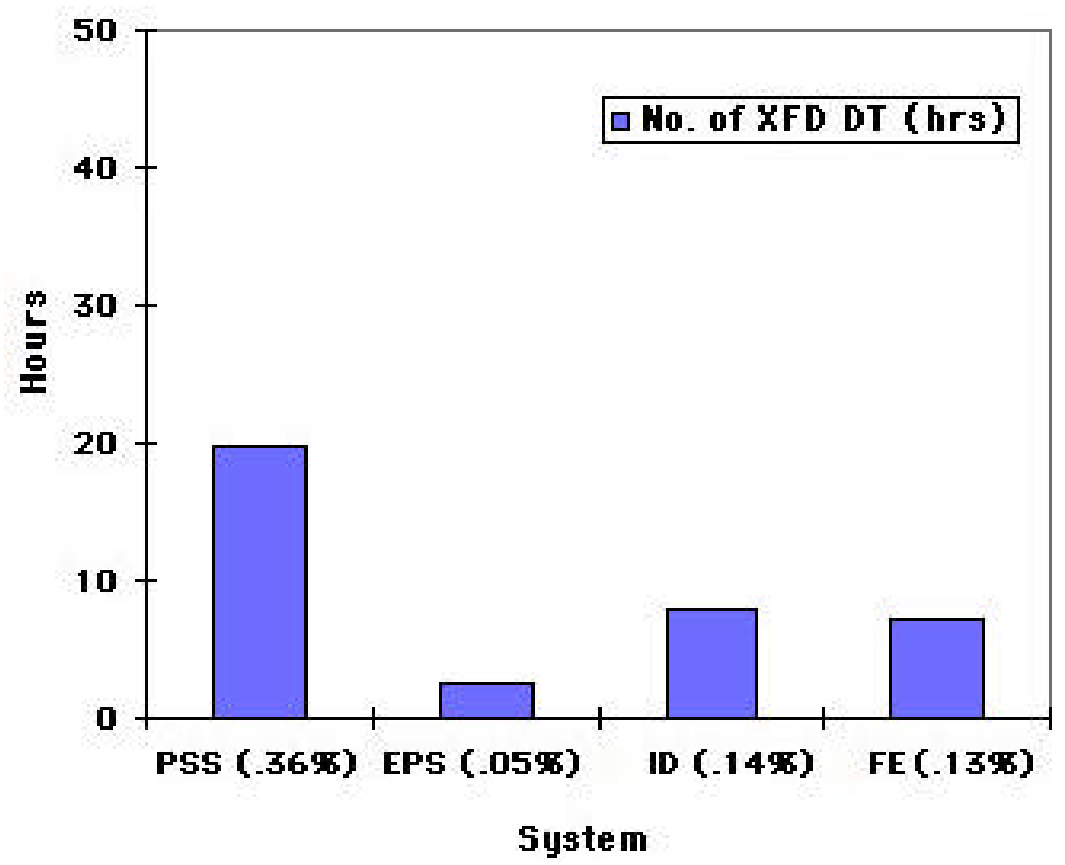

Fig. 2.5 System/component failures impacting beamline operations in delivering 5496 hours of user beam time.

caused high pressure levels that tripped setpoint-actuated interlocks. The most significant failure occurred in March 1998, when a ratchet-wall collimator developed a major leak taking an entire beamline off-line (see sections 2.6.6 and 5.2.2).

- Failures reported for the EPS system are usually due to problems, such as vacuum or water systems, that cause an EPS trip. Since the APS became operational in December of 1996, the FE EPS system has never failed as an operational system.

\subsection{Maintenance}

The maintenance program of the XFD operational systems consists of three parts:

1) Preventive maintenance includes tasks performed on a scheduled periodic basis to prevent failures while equipment is in use. This is accomplished in part by constantly monitoring equipment performance via the EPICS data logs. Maintenance schedules of this nature are driven mainly by beam run-time schedules due to the fact that much of the work can only be performed during shutdown periods. In addition, as shutdown time decreases, it becomes 
critical to optimize the amount of work performed. Undulator maintenance is a prime example of this.

2) Reliability-centered maintenance includes a scheduled maintenance program that increases the availability of an item of equipment by identifying failures or potential failures before they degrade equipment effectiveness. Examples of this type of maintenance are the semi-annual PSS validation and door maintenance program.

3) Emergency maintenance includes accidental failures for which a good inventory of spare parts is maintained.

\subsection{Beamline Operations}

\subsubsection{Introduction}

The Beamline Operations Group is responsible for reliable operation of all FEs and IDs. In this effort, the Group regularly uses the expertise of personnel in the Insertion Devices Group and the Beamline Engineering Group in XFD. The Beamline Operations Group is also responsible for providing technical support to users. All the Phase-1 40 FEs and 20 IDs were installed. In addition, the ASD diagnostic beamline, sector 35, was commissioned with a special undulator and a different type of FE. During the past year, of the original 20 IDs installed, 19 provided radiation to the beamlines on the experiment hall floor. Also of the 20 bending magnet beamlines, 12 provided radiation to the beamlines on the experiment hall floor. This past year was mainly devoted to maintenance and upgrades of existing FEs and IDs.

\subsubsection{Insertion Devices}

Currently, a total of 22 IDs are installed in 21 sectors of the storage ring. Most devices are $3.3-\mathrm{cm}$-period undulators, 2.4 meters long. Sector 2 has two devices installed, one $3.3-\mathrm{cm}$-period undulator and one $5.5-\mathrm{cm}$ period undulator. Sector 3 has a $2.7-\mathrm{cm}$ period undulator. Sector 11 has an elliptical multipole wiggler (EMW). Sector 14 has an 8.5-cm-period wiggler, and sector 35 has a 1.8-cm-period 4.5-meter-long undulator.

The ID vacuum chamber in sector 3 was replaced with a small vertical aperture chamber with a maximum vertical opening of $5 \mathrm{~mm}$ and an external vertical size of 8 $\mathrm{mm}$. This change has enabled the $2.7-\mathrm{cm}-$ period undulator to reach a gap of $8.5 \mathrm{~mm}$, which corresponds to a first harmonic energy of $5 \mathrm{keV}$, enhancing the capability of the sector 3 beamline.

All ID vacuum chambers have a transition chamber at either end, which allows the conversion from the standard 40-mmaperture storage ring chamber to the $8-\mathrm{mm}$ ID chamber. Internally, these transition chambers are made of copper and are water cooled as they are exposed to a significant heat load during normal operation due to radio frequency (RF) heating. Recently on some sectors there was a significant pressure drop across these cooling lines. The technique developed to clean FE components was adapted to clean these absorber cooling lines. In addition the cooling circuits were reconfigured to be part of lower flow circuits. 
Upgrades to the IDs were undertaken to enhance their capabilities and to increase their reliability. All IDs, with the exception of the EMW in sector 11, were retrofitted with new machine protection system switches and actuators. This arrangement allows the switches to trip at a gap of $50 \mathrm{~mm}$ and stay tripped at any gap larger than $50 \mathrm{~mm}$. The upgrade provides confirmation that the gaps are effectively "open" without having to fully open the devices to $200 \mathrm{~mm}$. This capability has effectively reduced the storage ring refill times by nearly $50 \%$. During the past year, many of the devices began stalling at moderate speeds. To overcome this problem, the stepping motor drivers were upgraded from 8-amp to 12amp capacity. More than half the sectors are currently equipped with the new stepping motor drivers. The higher current drivers allow the existing ID motors to produce more torque, resulting in more reliable ID operation, faster acceleration, and higher opening and closing speeds. This is a major benefit to users who are "scanning" an ID, moving the gap to a new energy, and taking data every few seconds. Linear potentiometers were installed on all devices as a redundant method of gap measurement. They can be used as input for the EPS. They are also used as inputs for a gap monitor system in sectors with multiple IDs, such as sector 2, where two devices are installed and the FE components cannot handle the power of both devices simultaneously at fully closed gaps.

Extensive modifications were made to the ID control software for more reliable operation, user friendliness, and easier revision when new features are added. A beamline commissioning limit was established, allowing users to specify a minimum gap for ID operation for protection of their own beamline and experimental components. This software limit is independent of limits established for protection of the ID, ID vacuum chamber, FE components, and experiment stations. A "deadband" was added to the control program to allow users to specify a tolerance on the desired gap. This is especially useful for scanning of the ID in small steps over a certain energy range. Time is not wasted in extremely precise positioning of the ID if lower precision is acceptable for the experimental demands of the user. The gapto-energy conversion in the ID control software was enhanced. New code is used to correct the energy calculation to correspond more closely to the x-ray energy. The finite beam size is taken into consideration in calculating the energy for any specific harmonic and gap. Users were given the option of specifying the required harmonic (maximum $7^{\text {th }}$ order) for energy readback and control values.

\subsubsection{Front Ends}

Currently there are 41 FEs installed, of these, 31 have provided radiation to the experiment hall floor and 20 (including sector 35-ID) are for ID beamlines. During the course of the last few years of operation, certain inherent problems have surfaced. In addition to resolving these problems, upgrades and modifications have been performed.

The EPICS interface to the FE controls was enhanced during the past year. Control of the all the devices is now possible via EPICS. At the present time, floor coordinators and operators can reset faults and open and close vacuum valves remotely from their workstations. Access security was implemented to avoid unwarranted operation of devices. In addition the FE system 
manager can control all the vacuum pumps. Residual gas analyzers (RGAs) in all the FEs have been interfaced to EPICS, allowing for remote control and constant monitoring of the RGAs, which is useful for diagnosing vacuum problems. Alarm handlers have been implemented in EPICS for all FE systems. Advance warnings (in the form of e-mail to pagers) warn of potential faults, so preventive action can be taken to rectify the problem.

In order to meet the CATs' needs, seven of the ID FEs and one BM FE have been retrofitted with differential pumps for windowless operation of the beamlines. Windowless operation allows the beamline to utilize lower energy $\mathrm{x}$-rays and reduce scattering. Also reduction of flux/brilliance due to $\mathrm{x}$-ray absorption in window assemblies (containing a set of filters) is avoided. All these windowless beamlines have an RGA just downstream of the differential pump. Work on interlocking and alarming on RGA signals is underway.

Problems have occurred in recent months on the FE vacuum systems. The problems were identified to be one of two types. The vacuum fast valve has sprung vacuum leaks in several FEs. The vacuum leak occurs at the wire seal located in the ring surrounding the conflat flange. Similar problems have been reported by other facilities when they baked their valve to $250^{\circ} \mathrm{C}$. (At the APS, it was baked only to $150^{\circ} \mathrm{C}$.) The seals have been replaced in some of the FEs, which requires venting half the $\mathrm{FE}$ and subsequent baking. The other vacuum failure has been attributed to the viton seal in the FE exit valve located just outside the shield wall. This valve was chosen for its characteristic particle-free nature, fast closing speed, and 1-million-cycle lifetime. Failures were observed in the form of slow leaks through the seal. Some of the valves were sent back to the manufacturer for analysis. Based on these results, the manufacturer has modified the valve and has rated the valve only for 100,000 cycles and longer closing times. Thus, all future replacements will be made with series 10 valves, which are 1/3 the cost.

One common failure mode during the past year has been due to the FE cooling water system. This failure triggers the protection system to halt operation of both the beamline and the storage ring. The problem has been traced to the pressure-sensitive transducers. The manufacturer has acknowledged the problem in these devices and has retrofitted them whenever the devices were taken out of the system. In addition it was noticed that most of the components in the FE were not meeting the design specification for coolant flow. All mesh-based components in the FE were subsequently cleaned by a technique developed locally. The cleaning cleared the blockages in the cooling channels and brought flow rates to their design values. The output of the pressure transducers was interfaced to the EPS system. Each flow or differential pressure has both an upper- and lower-limit alarm that can trigger an inhibit for operation of beamline/storage ring. The upper-limit alarm was found unnecessary and was removed. As an added preventive measure, EPICS alarms were set to notify staff when the flow/pressure was within $30 \%$ of the trip level. All these enhancements have resulted in increased reliability of this system.

Each FE has two X-ray beam position monitors (XBPMs) installed. These devices can measure the position and angle in both the vertical and horizontal planes in the case of ID beamlines and in the vertical plane in BM beamlines. All the XBPMs in the FEs were instrumented. The current signals 
originating from the XBPMs were amplified and converted to voltage and fed to a fast ADC processor. The digital output of the ADC processor is transmitted via fiber optic to a VME-based receiver module. The receiver module is located in the storage ring feedback input output controller (IOC) system. High-speed XBPM data are available for the storage ring feedback system for future closed-orbit feedback systems. On 9 of 20 ID front ends, a digitalsignal-processor-based XBPM calibrated system has been implemented. Once calibrated, this system reports the $\mathrm{x}$-ray beam position in real units independent of ID gap or storage ring current.

\subsubsection{User Beamlines}

As the APS moves towards a mature operational state, the Beamline Operations Group has started providing support to the users on a regular basis. Most of the beamlines use processed water for all their component cooling needs. The processed water for each sector is a closed-loop system consisting of a deionized (DI) water plant located in the mechanical mezzanine and a distribution system for each station. Most of the CATs have chosen the DI water plant designed by the APS. Beamline Operations has taken on the responsibility of commissioning and subsequent maintenance of the DI water systems for the CATs. The CATs are provided round-the-clock coverage for emergency repair service on their DI water plants.

The undulator beam delivered at the APS has very high power density and total power. Most of the beamlines use a monochromator as their first optics to handle this powerful beam. Liquid nitrogen is the coolant of choice for the monochromator, as it keeps the optics distortion at a minimum while providing the necessary cooling capacity to handle the high head loads. Most of the beamlines use an Oxford Instruments liquid nitrogen pump for pumping liquid nitrogen through the monochromator crystal. The Beamline Operations Group has taken on the responsibility for providing emergency service and routine maintenance of these user pumps. Spares needed for the service of these pumps are available to meet emergencies.

\subsubsection{User Interfaces}

At the Advanced Photon Source, all controls are standardized with EPICS. This system consists of equipment interfaced to VMEbased hardware. The VME crate (normally called the IOC) talks to the computers via the Ethernet. With EPICS, access to the controls is available from any computer located on the same network. This scheme has a great degree of flexibility.

Most of the CATs have chosen EPICS as the control system for their beamlines. Beamlines routinely need various information from the APS control system. A multi-prong approach was taken to disseminate the relevant information to the user at the APS. The Web has been used as one platform for providing information about the machine. This information is not in real time. The Web platform is used mostly for archived data and for informative purposes. A cable TV system with 14channel capability was installed around the storage ring, and the information is also distributed to the rest of Argonne including the Guest House. At the current time, only 2 of the 14 channels are being used. The data are in real time providing information about the storage ring operating status on one 
channel and the beam pinhole image and size on another channel. A plan is underway to provide other desired information on the rest of the channels in the future.

Users requiring data in real time cannot use either of the above-mentioned schemes owing to the need for security and isolation of the control system. An EPICS interface gateway has been developed to overcome this limitation. A high-speed Unix-based Ultra Sparc system was set up with bridges to the two networks. The gateway provides any data that are available in the control system to the users as read-only on a realtime basis. In addition the gateway is used in specific cases to provide specific users access to control equipment in the control system, for example, the IDs. For each of the ID beamlines, users can control their ID from designated computers.

The EPICS gateway has some limitations. Due to the large number of beamlines and users, the performance of the gateway can degrade and will not be able to provide data at the same rate as available on the control system. Hence, for the present, the gateway is throttled down to avoid any down time, while new solutions are sought to mitigate this limitation.

A new system is being implemented using a fiber optic link. Each beamline has a dedicated multiconductor fiber optic cable installed from their respective ID control system IOC (VME-based). The intent of this system is to provide a direct link from the APS control system to the beamline control system while making sure that security is not compromised. The receiver module is located in the beamline control system IOCs. The data flow is unidirectional from the APS control system to the beamlines.
All CATs were provided with a stand-alone receiver module. This module has displays in the form of LEDs and an alpha-numeric display for current. In addition, it has outputs in the form of voltage, current loop, and frequency, corresponding to the stored beam current. All LED displays have a corresponding transitor-transitor logic (TTL) signal for various bilevel signals like injection status, shutter status, orbit correction status, etc. In addition, the revolution clock signal $\left(\mathrm{P}_{0}\right)$ is available on these units for users performing timing experiments.

A VME-based receiver module has been successfully tested in one of the sectors. The VME receiver module can be located at any of the beamline station IOCs. The fiber optic link is used by this receiver to provide the data to the user's experiment. The data from the APS control system are available to the users seamlessly. Installation of the module is under way for the remaining sectors. The VME-based receiver module has sectorspecific information available to the users directly in their IOCs. Some of these signals are related to ID parameters, FE beam position monitor (BPM) signals and storage ring particle BPM signals for both the ID and BM beamlines, as well as FE shutter status. In addition, all the common signals, like storage ring current, injection status, etc., are also available.

The fiber optic information system helps relieve the load on the EPICS gateway. In addition, the users have the advantage of having the data available directly in their IOCs for seamless integration to their experimental setup. This system preserves the high level of security to the APS control system and also distributes the load uniformly around the ring to various IOCs. Plans are under way to provide the users 
with bunch clock signals for timing experiments.

\subsubsection{Sector 5 Front-End Problem}

At the APS, our first major failure of a FE took place in March 1998, disabling the sector 5-ID beamline from operating for a period of about three weeks. During the maintenance period following the operating cycle, the FE was fixed and the beamline was put on-line. The CAT lost about three weeks of useful user beamline operation time.

In early March, whenever the CAT closed the device to small gaps there was a noticeable rise in the pressure in the FE vacuum system. As time passed, the vacuum further degraded, and the EPS disallowed the opening of beamline shutters. During the next available access to the storage ring, vacuum leak checking identified the problem as the ratchet-wall collimator. The ratchet-wall collimator tube was found to be bent. At the next scheduled maintenance time, the FE was surveyed, and all components downstream of the slow valve were removed. The second photon shutter and the safety shutter were found to be in good condition. The ratchet-wall-collimator vacuum chamber was bent, and a small crater was found about 3 inches from the upstream side of the collimator tube.

After extensive investigation and review, it was determined that the wall collimator tube should not be constrained at both ends because the constraint prevented the wall collimator tube from expanding and contacting linearly. If both ends are constrained, the tube can bend at the least constrained point. Once the tube started bending, it was close to the beam and in a posistion to be exposed to heating from the beam, which in turn resulted in further bending. Eventually the bottom surface of the collimator tube was in the path of the direct undulator beam, which caused a crater and the subsequent vacuum failure.

At the current time, the cause of the contraction of the collimator tube is not clear. However it was clear that unconstraining the collimator tube would have averted the failure. As a preventive action, all ratchet-wall collimators were modified to allow for dimensional changes in the tube.

\subsection{Interlock Systems and Instrumentation}

The Interlock Systems and Instrumentation (ISI) Group is responsible for generating and/or supporting the design, installation, testing, and maintenance of the PSS, EPS, and FE instrumentation. This includes any and all documentation, testing, and fieldwork required for supplying the XFD with high reliability systems. Each system consists of numerous subsystems that are high reliability and fail safe. The PSS is a redundant interlocked system that monitors personnel access into beamline enclosures. The EPS is an interlocked system that reduces risk of damage to $\mathrm{FE}$ beam transport equipment. The group is organized into three functional blocks. The interlock system design section provides interlock systems requirements, scheduling, budget/cost development and control, drafting, and project management support. These systems are designed to applicable codes, orders, and standards for such systems. Software is developed in the 
software development section under the software development plan and conforms to the Laboratory's Software Quality Assurance Plan. The hardware function in the hardware design section relates to the design, systems requirements, scheduling, budget/cost, drafting, and project management support of FE instrumentation.

\subsubsection{The APS Personnel Safety System}

\section{Introduction}

The APS is designed with the capability to operate at least 70 beamlines concurrently. Each beamline includes several shielded experiment stations. Personnel access into these stations is controlled during beamline operation via the APS/XFD PSS. The PSS is an engineered safety system that interlocks personnel access to these stations with x-ray beam-off conditions via beam shutter operation and, if required, storage ring operation.

Although there are a variety of beamline designs that reflect the types of experiments being done at the APS, basic PSS configuration and control functions remain the same. If required, specialized user control panels are incorporated into the standard library of PSS hardware.

The PSS is designed to comply with accelerator safety standards in DOE Orders and other relevant good practices for accelerator facilities. Among the requirements derived from the above criteria, to which the PSS conforms, some of the more important items are as follows:
- The system is designed to be fail safe, so that common failure modes leave the PSS in a safe, beam-off state.

- The designs incorporate redundant protection, ensuring that no single component or subsystem failure leaves the PSS in an unsafe condition.

- Provisions for testing are included, so the proper component and system function may be verified.

- Access and egress controls are incorporated so that personnel are not exposed to $\mathrm{x}$-ray radiation. These include emergency shut-off devices, status signs, search and secure procedures, and emergency exit mechanisms.

- A strict configuration control system protects documentation, circuits and software against unauthorized and inadvertent modification. Critical devices are clearly labeled to note that tampering is strictly forbidden.

\section{PSS Configuration Management Plan}

Safety experts consider rigorous configuration management (CM) mandatory for any organization responsible for developing safety critical systems. Thus, $\mathrm{CM}$ is essential to the mission of the ISI Group. XFD has implemented CM that provides the mechanism whereby assurances can be made that the appropriate system is being used. 


\section{PSS Installation Status}

Although the number of new installations has not increased dramatically over the last 16 months, the biannual requirement for revalidation of the PSS systems required a continued level of support. The number of user stations instrumented with the PSS is shown in Figure 2.6. The figure also shows the number of validations performed during this period and summarizes the planned future activities.

\section{Support for PSS Validations}

Scheduled periodic validations of each beamline PSS afford APS users the opportunity to request operational changes in these systems. Thus, over $95 \%$ of the nontrivial PSS validations conducted during a year involved either system or user changes, which require both development and configuration management support within the ISI Group. Typical technical support needed for each system change in a given beamline PSS includes engineering support for developing, reviewing, testing and documenting proposed design changes. Additionally, technician support is needed for installation and testing.

\section{PSS Improvements in Support of Operations}

Improvements in the current PSS design are planned in the areas of operational reliability and testing efficiency. The present PSS design incorporates permissive protective logic and some command/control functionality. The new PSS design will not have command/control features in its safetycritical portion, as does the current PSS design. Thus, the new PSS design will conform to all relevant mandated DOE and

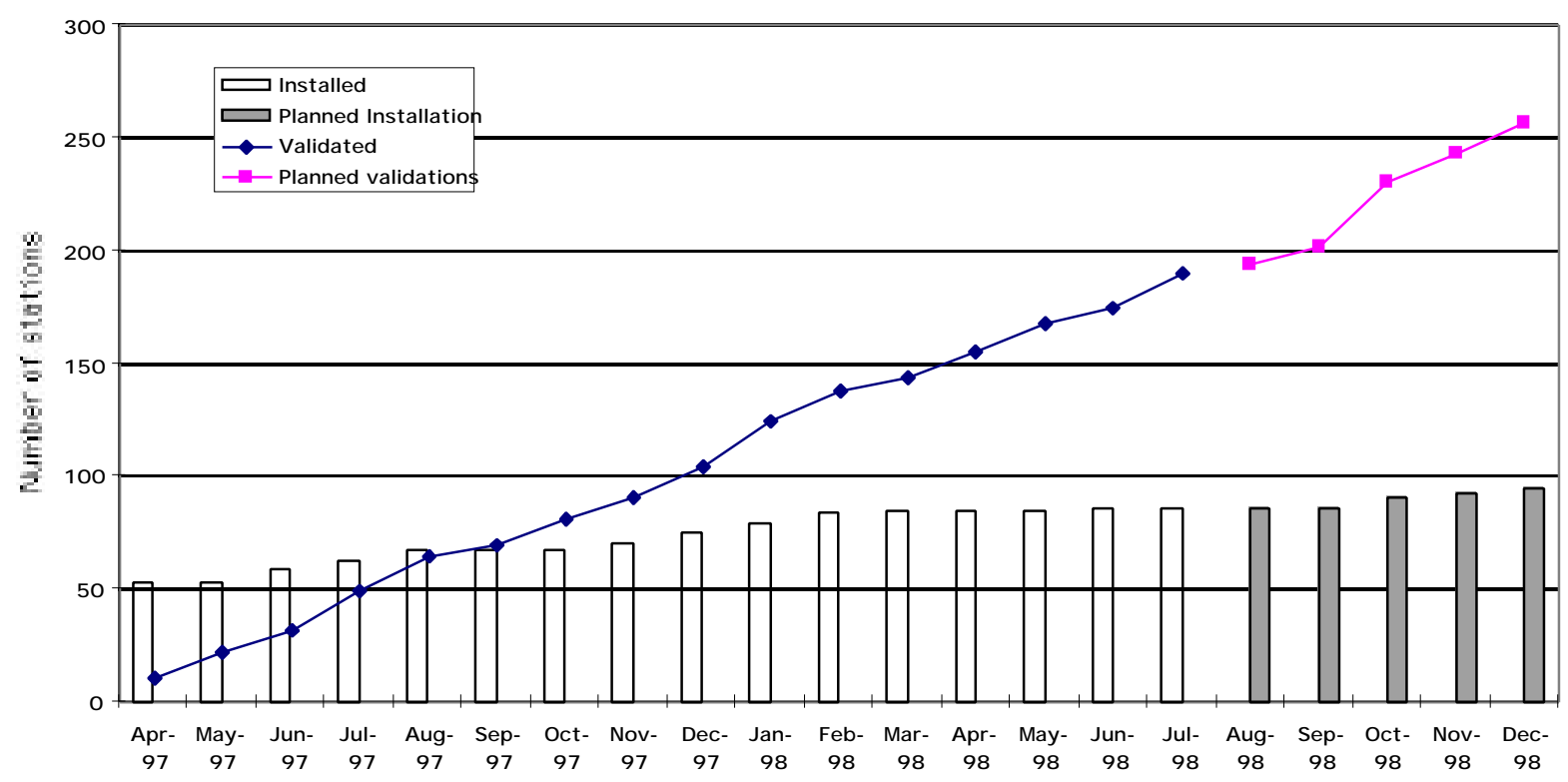

Fig. 2.6 Number of PSS installations and revalidations (completed and planned). 
ANL safety design criteria and will provide better operational performance, with virtually exhaustive safety test coverage while reducing the testing duration. Ultimately this means a more seamless PSS interface for the APS users while providing more enhanced personnel access safety.

\section{PSS EPICS Interface}

Based on run time experience, it is evident that the PSS must have significant diagnostics available to minimize user beamline downtime.

Furthermore, quick accurate trouble analysis, useful data logging of PSS parameters, and user "remote" operation/monitoring of some PSS parameters not only enhance beamline operating efficiencies but provide essential information for preventative maintenance. These fundamental diagnostic and logging tools are best provided via an EPICS interface with the PSS.

The needed diagnostics and logging capabilities will be provided by configuring the PSS EPICS so there is one PSS EPICS IOC per beamline.

\subsubsection{The Equipment Protection System}

\section{Introduction}

The APS has presented a number of design challenges in protecting $\mathrm{FE}$ and beamline components from being damaged by thermal loads produced by high-brilliance hard $\mathrm{x}$-rays. Another major goal is to ensure that the storage ring vacuum is not compromised under any vacuum-failure scenario in the FE or beamline.

The FE Equipment Protection System (FEEPS) monitors and controls devices located in the beamline FEs. Actions taken depend largely on the severity of the fault, ranging from merely setting an alarm, to closing shutters and valves, to inhibiting stored beam. One of the major considerations driving system design was to limit beam aborts thus contributing to higher operating efficiency of the facility.

Fail-safe principles are incorporated into the design, and the system will lapse into a predetermined safe condition (de-energized to trip) following a failure, including loss of power, air-pressure drop, drop in water flow, shorted outputs, and open circuits.

\section{System Overview}

Programmable logic controllers (PLCs) are used to handle all system monitoring, control, troubleshooting, and reporting functions. PLCs allow for the design of a very advanced interlock and control system that can handle a large number of distributed I/O points. Each FE is provided with an autonomous equipment protection system that monitors the following parameters: cooling water flow and differential pressure, vacuum sensors, pneumatic pressure, photon and safety shutter positions, positions of vacuum valves, and status of the systems to which the FEEPS interfaces

In order to isolate different power systems, all interfaces between the FEEPS and other systems and subsystems are implemented 
through relay contacts. These interfaces are listed in Table 2.3.

\section{Installation Status}

\section{Overview}

Currently 41 FEEPS are instrumented, commissioned, and in operation. This includes 40 systems planned for the Phase-1 installation period, as well as the 35-ID FEEPS. Phase-1 installation data (in percent) is reflected in Fig. 2.7.

Additional systems are being brought on line on a regular basis. A FEEPS system for

Table 2.3 System Interfaces and Functions

\begin{tabular}{|l|l|}
\hline \multicolumn{1}{|c|}{ System } & \multicolumn{1}{c|}{ Signal Functions } \\
\hline $\begin{array}{l}\text { Beamline } \\
\text { PSS }\end{array}$ & $\begin{array}{l}\text { Monitor shutter-open positions } \\
\text { Control of photon shutters }\end{array}$ \\
\hline $\begin{array}{l}\text { Insertion } \\
\text { Device }\end{array}$ & $\begin{array}{l}\text { Monitor gap for 'max open' status } \\
\text { Control emergency gap open }\end{array}$ \\
\hline SR ACIS & Monitor shutter-closed positions \\
\hline Vacuum & $\begin{array}{l}\text { Monitor SV, FV, CC1 and CC2 status } \\
\text { Control SV and FEV } \\
\text { Permit vacuum controller operation in } \\
\text { local mode }\end{array}$ \\
\hline Beamline \\
EPS
\end{tabular}

the 35-BM FE will be instrumented and validated in the fourth quarter of 1998.

All 41 systems have been commissioned at least up to the first photon shutter (PS1). However, full implementation of the FEEPS is governed by the beamline installation pace. It is the responsibility of the XFD personnel to maintain a high level of reliability and availability of the FEEPS. To accomplish this, in addition to the initial system validation of proper operation, a full functional revalidation of each FEEPS system is being conducted at twelve-month intervals.

\section{Reporting and Control}

Status information of all FEEPS systems is incorporated into the EPICS-based APS control system. This allows monitoring of the interlock system from any networked PC, X terminal or Unix workstation. In addition, all system trips are captured to facilitate troubleshooting and performance analysis.

Graphical displays include an overall view of the storage ring and FEs, as well as zoomin screens for each interlock and control system. The information available is comprehensive, ranging from the upper level summation tables down to the individual field device sensors.

The graphical user interfaces also make it possible to control FE shutters and vacuum valves, as well as reset latched trips remotely. This control capability is under configuration control and is only available to authorized personnel. 


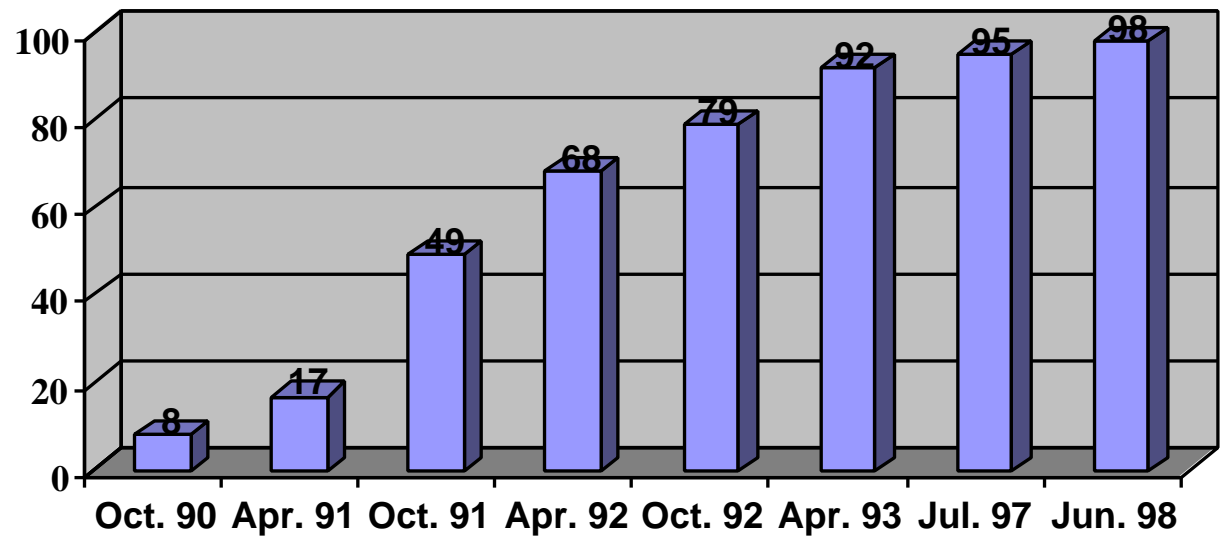

Fig. 2.7 Phase-1 FE Equipment Protection System installation progress (in percent).

\section{New Initiatives}

A number of important system upgrades are planned. Some are listed below.

- Place PLC processors on DH+ bus. This will allow PLC access for program download and troubleshooting from a "single" location.

- The fast valve (FV) is triggered to close by a dedicated cold cathode gauge. The FV trigger to close causes an immediate beam abort. Equip ID FEs with a redundant vacuum gauge, so that both sensors will have to indicate pressure rise for the FV to close.

- On bending magnet FEs, don't dump stored beam on FV trigger - PS1 will close to protect the valve.

- Enhance EPICS displays by adding system schematics for real-time display and control.

\section{Operations Experience}

The operation of the FEEPS began in December 1994. The last three and a half years have provided valuable operating experience. Some process variables and time delays were fine-tuned. Most noticeably, we have decided to do away with temperature interlocks and to rely only on the doubly redundant cooling water interlocks. The main reasons for not interlocking on temperature are the low level of reliability, as has been observed at other light source facilities, and the potential for nuisance trips.

During the more than 3.5 years of operation, not a single storage ring beam dump was caused by the FEEPS. The trips were all for "legitimate" reasons, most due to the flow rate dropping out of the predetermined range, and some resulting from the vacuum system faults. In all cases, the FEEPS responded the way they should, and there have been no unexplained trips. 


\subsubsection{Instrumentation}

\section{Instrumentation Improvement in Support of Operations}

A wireless communication network for efficient operations support is planned. The intent is to provide a portable computer with a wireless communication interface that can be used to assist in diagnosing PSS or FEEPS problems. This unit will supply system information, automated troubleshooting guidance and voice communications that can be carried to the system hardware in question on the experiment hall floor.

Engineering support is being provided for the following beamline instrumentation projects:

- High voltage solenoid pulser - the APS has need of a high-speed x-ray beam chopper to be used within a program devoted to time-resolved measurements. The required highspeed chopper will operate within a time window of $2.35 \mu \mathrm{s}$. Also, the beam chopper motor must have the speed, precision and stability necessary to phase lock the time window of the beam chopper to the revolution frequency of the storage ring and the variability to allow matching the beam chopper time window opening to the "bucket" pattern of the APS synchrotron radiation. An $80 \mathrm{~K}$ rpm motor from Speedring and associated feedback control is planned to implement this design goal.
- Engineering analysis of FE shutter operation using high-speed data acquisition systems interfaced to the FE EPICS control system.

\subsubsection{Controls}

Due to the flexibility provided by EPICS, all data are available at all times to anyone with access to the computer network. At the APS, we have a separate subnet with restricted access for all the control systems. All the IOCs are located on this subnet. This provides an added security from unwarranted access to the IOCs. With the location of a EPICS gateway, data from the control system are provided to other subnets.

In a typical FE, currently EPICS can only read and is not allowed to control. All controls for the FE have to be performed at a location on top of the storage ring. All vacuum pump and gauge controllers are interfaced with EPICS. The interface enables the ion pump current and the vacuum to be read continuously. All water flow and pressure systems are also interfaced to EPICS via the RS-485 interface available in the interface controllers. All data from EPICS are constantly logged. This provides for later analysis of specific trends. The constant monitoring of data provides us with flexibility for advance warning on failures, thereby preemptive action can be taken to avoid them.

The XBPM raw voltage signals for the current amplifier are interfaced to the control system via an RS-485 interface. The normalization of the raw signals is performed in the IOC. All signals, both raw and normalized, are available via EPICS. 
Insertion device control is also implemented with EPICS. The ID motor controllers are commanded by EPICS, and the encoders are used to read the precise position of the device. Using the EPICS gateway, added security control to the IDs is provided to specific users of a particular beamline.

The PSS and FEEPS operator/user interface (OUI) is provided for APS facility use. The remote OUI for PSS and FEEPS has the capability to interface with EPICS. User screens have been developed that graphically represent the PSS status, and the remote OUI does not control any PSS functionality.

\subsection{Experiment Floor Operations}

In March 1998, the APS floor coordinators became members of the Experiment Floor Operations Group. Their principal responsibilities remain the same: they provide the day-to-day technical support for the APS users. In addition to their support role, the floor coordinators provide the primary APS oversight of beamline operations. The coordinators' offices are distributed around the experiment hall, with two coordinators assigned to the four sectors that are associated with a specific LOM. Floor coordinators familiarize themselves with the operation of the beamlines within their areas of responsibility and have the authority to suspend operations if they feel that unsafe conditions may exist. Whenever the facility is operating or whenever beamlines are undergoing significant modification, a floor coordinator will be "on duty" representing the APS.

During the past year, five floor coordinators have been hired. The floor coordinator team is being built with personnel who are experienced in a variety of different aspects of the construction and operations of research facilities. In addition to the on-thejob training for new coordinators, a seminar series was organized to introduce the coordinators to some of the experimental programs at the APS as well as to develop a deeper understanding of the detailed operation of the APS technical systems. As the number of users increases, so does the variety of samples and experiments on the beamlines. The floor coordinator duties will expand to provide service in the areas of biological, radioactive, and chemical sample handling.

\subsubsection{Shielding Validation of the Experiment Stations}

The Experimental Facilities Division, in collaboration with the Health Physics Personnel from ANL's Environment, Safety and Health $(\mathrm{ES} \& \mathrm{H})$ Division, performs the shielding verification of all the user experiment stations in the presence of CAT personnel. The governing process for commissioning is documented and has been approved by the DOE. The CATs are informed immediately of any shielding deficiencies discovered during the enclosure commissioning. Activity on the beamline cannot proceed until the deficiency is mitigated. The CATs are allowed to proceed with commissioning activity of beamline instruments only after successful completion of shielding verification of the enclosures.

Shielding verification is done for bremsstrahlung, synchrotron radiation, and neutrons. So far, 45 first optics enclosures/ white-beam stations and 33 pink/monobeam stations have been verified at the APS. 


\subsubsection{Measurement of Radiation Dose Received by IDs}

The radiation dose received by the magnetic structures of the IDs was monitored for each run. Radiochromic films were placed at various locations by each of the IDs before each run. The accumulated dose shows that the maximum dose received by the IDs until now is in the range of a few Mrads (5-10 Mrads).

A program to study the degradation of the permanent magnets as a result of high radiation doses is in progress. $\mathrm{Nd}-\mathrm{Fe}-\mathrm{B}$ permanent magnet samples will be irradiated with $\mathrm{x}$-rays, gamma rays, and neutrons under controlled conditions. The total absorbed dose in each case will be measured by an appropriate high-dose dosimetry technique, such as radiochromic films or photoluminescence dosimeters. The temperature of the magnet during irradiation will be closely monitored. The first batch of magnets will be irradiated by $\mathrm{x}$-rays at the 20-BM beamline during Run 4 of 1998. Gamma irradiation will be measured at the high-dose Co-60 gamma cell $(9 \mathrm{KGy} / \mathrm{h})$ at NIST.

\subsubsection{Measurement of Bremsstrahlung Absorbed Dose in Tissue Phantom}

High-energy electron storage rings generate energetic bremsstrahlung photons through radiative interaction of electrons (or positrons) with the residual gas molecules and other components inside the storage ring. At the APS, where the beamlines are channeled out of the storage ring, a continuous bremsstrahlung spectrum, with a maximum energy of the positron beam is present. Measurement of the primary bremsstrahlung energy spectra has been conducted at the APS ID beamlines and has been reported in the Experimental Facilities Division Progress Report 1996-97 (ANL/APS/TB-30).

A polymethyl methacccrylate (PMMA) slab phantom of $30 \mathrm{~cm} \times 30 \mathrm{~cm} \times 30 \mathrm{~cm}$ is used to measure the absorbed dose by bremsstrahlung radiation in tissue equivalent material. Thermoluminescent dosimeters (TLDs) (LiF, TLD-700) are used to measure the dose. The PMMA slabs were placed in the bremsstrahlung beam in the FOE of the 15-ID beamline. The TLDs measured the absorbed dose in the longitudinal and the transverse directions in the phantom. The preliminary results indicate a maximum normalized absorbed dose rate of $1.0 \times 10^{-2}$ $\mathrm{mGy} / \mathrm{h} / \mathrm{nT} / \mathrm{mA}$. Detailed analysis of this experiment is underway.

\subsubsection{Photoneutron Dose Measurements in the First Optics Enclosures}

Bremsstrahlung of sufficiently high energy $(>10 \mathrm{MeV})$ can interact with beamline components, such as beam stops and collimators, generating neutrons of varying energies. There are three main processes by which neutrons may be produced by the high-energy bremsstrahlung photons: giant resonance dipole decay $\left(10<\mathrm{E}_{\gamma}<30 \mathrm{MeV}\right)$, quasi-deuteron production and decay $\left(50<\mathrm{E}_{\gamma}<300 \mathrm{MeV}\right)$, and a photopion produced cascade $\left(\mathrm{E}_{\gamma}>140 \mathrm{MeV}\right)$. At the APS, where bremsstrahlung energy can be as high as $7 \mathrm{GeV}$, production of neutrons invarying yields is possible from all three processes. 
A simultaneous measurement of bremsstrahlung and the corresponding photoneutron dose rates from different targets like $\mathrm{Fe}, \mathrm{Cu}, \mathrm{W}$, and $\mathrm{Pb}$ was conducted at the FOE of the APS beamlines to obtain the photoneutron dose rates as a function of bremsstrahlung power. An
Andersson-Braun remmeter that houses a sensitive ${ }^{3} \mathrm{He}$ detector is used for neutron dose measurements. The dose equivalent rates, normalized to bremsstrahlung power, are measured with the four targets. The results are given in Fig. 2.8. 

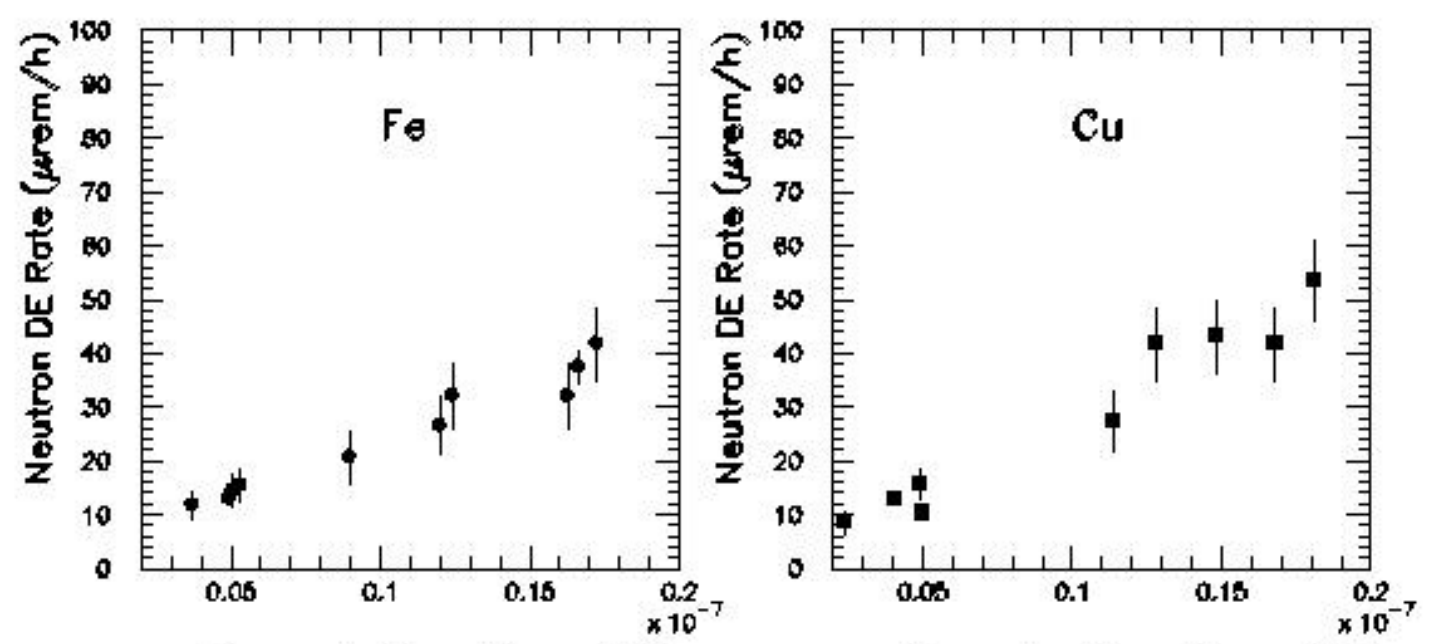

Bremsstrahlung Power ( $\mathrm{KW}$ )

Bremsstrahlung Power ( $\mathrm{KM}$ )

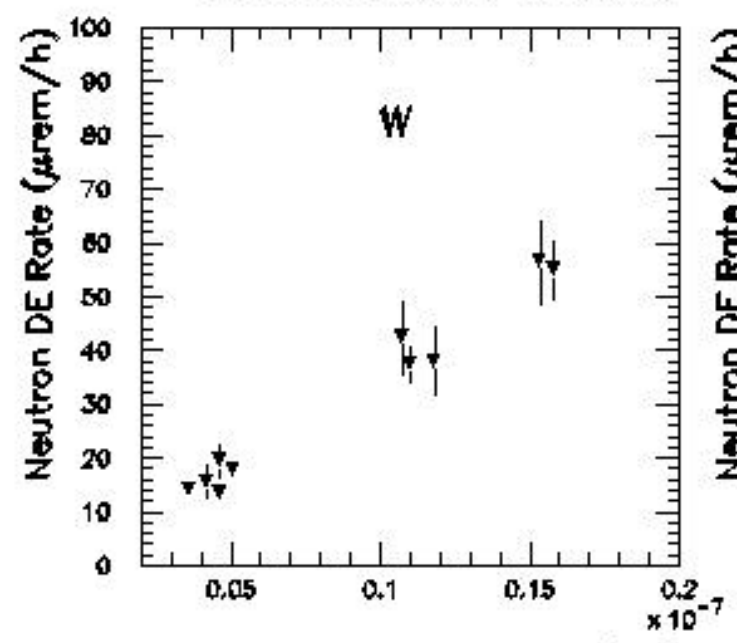

Bremsstrahlung Power (kW)

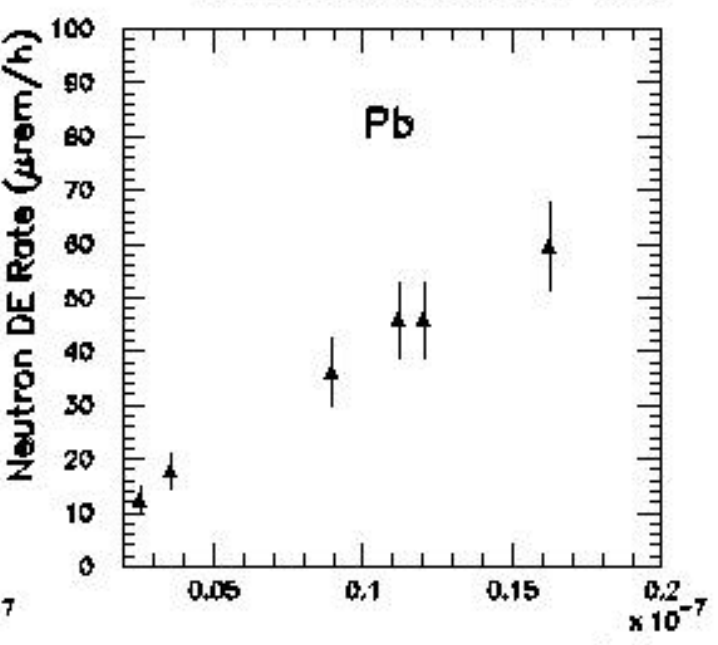

Bremsetrahlung Power (kw)

Fig. 2.8 The photoneutron dose equivalent rate, measured $80 \mathrm{~cm}$ lateral from each target center, as a function of the incident bremsstrahlung power. 


\subsection{APS Users}

At the APS, users fall into three categories: (1) Collaborative Access Team (CAT) members, (2) collaborators or colleagues of CAT members, or (3) Independent Investigators (IIs). CATs are responsible for building and operating the beamlines in one or more sectors; thus, CATs comprise not only scientific research members, but also beamline construction and operations staff. Collaborators and IIs come to the APS solely to conduct research; their home institutions have no formal affiliation with the CAT on whose beamline they work.

\subsubsection{Collaborative Access Teams}

The CATs are selected through a comprehensive, peer-review proposal process developed by the APS in 1989 and described in last year's Experimental Facilities

Division Progress Report 1996-97 (ANL/APS/TB-30). By the end of 1997, 14 CATs had been approved, accounting for 20 sectors. Figure 3.1 shows the distribution of these 20 sectors by primary discipline. Subsequently, an additional six Letters of Intent have been received and approved. Full scientific proposals have been received for all of them. Five of the proposals were approved by the Program Evaluation Board (PEB) prior to September 1998, which has resulted in allocation of two additional sectors. If all requests for new sectors receive approval, a total of 26 sectors will be committed; the projected distribution by discipline is also shown in Figure 3.1. (Appendix 4 provides a detailed record of the entire CAT review process to date.)

During their first year of operation, CATs (and their collaborators) are entitled to use $100 \%$ of the available beam time. (A CAT

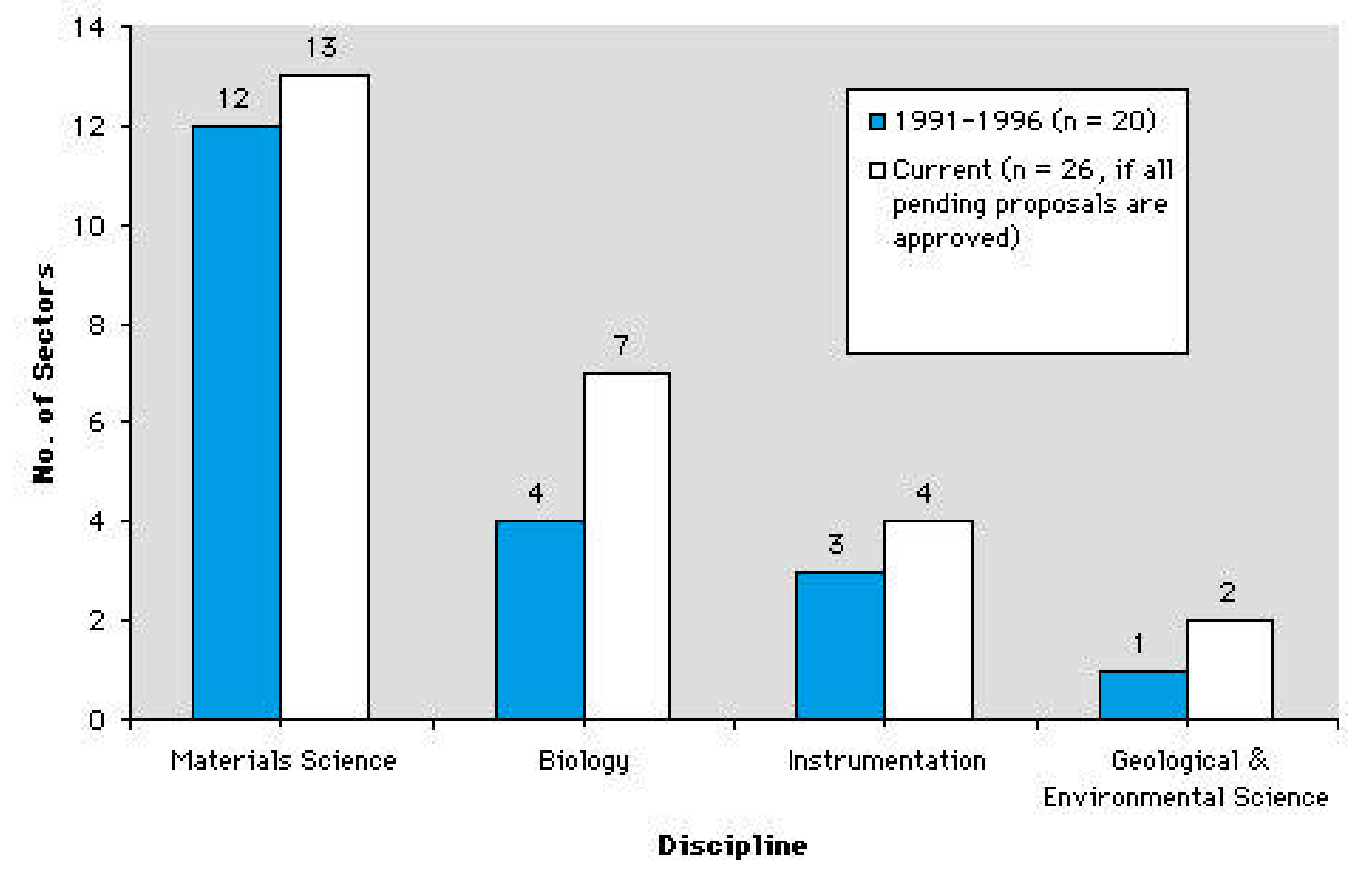

Fig. 3.1 Distribution of APS CAT sectors by primary scientific focus (as of 7/30/98). 
specifies when its beamlines-or portions thereof-are officially operational.) After the first year, they are entitled to $75 \%$ of the beam time and must make the remaining $25 \%$ available to IIs selected through a peerreview proposal process. Each CAT develops its own process, which must be reviewed and approved by the APS. As of September 1998, only SRI-CAT has begun an official II program.

\subsubsection{Independent Investigators/Collaborators}

On April 1, 1998, SRI-CAT officially began its II program. Two other CATs (IMCACAT and UNI-CAT) have APS-approved II plans in place but have not yet officially declared their beamlines operational. To date for SRI-CAT, 10 II proposals have been received, reviewed, and accepted. All other CATs, however, have been conducting commissioning-related experiments with CAT members and collaborators.

\subsubsection{User Community Description}

As of July 30, 1998, the APS user community comprised 1070 persons. (An APS user is defined as an individual who has been at the APS to conduct hands-on work, has completed a general/safety orientation, and has received a user badge.) Of the 1070 APS users, $64 \%$ are CAT members, and the remaining $36 \%$ are either IIs or collaborators. Figure 3.2 shows the growth of this community during the past five years. The first five users officially arrived by April of 1994; during that first year, 35 individuals were oriented and given badges. In contrast, from January 1 through July 30, 1998, 395 new users completed this process. Figure 3.3 shows the distribution of current APS users by institutional affiliation, Figure 3.4 by primary research funding source, and Figure 3.5 by discipline.

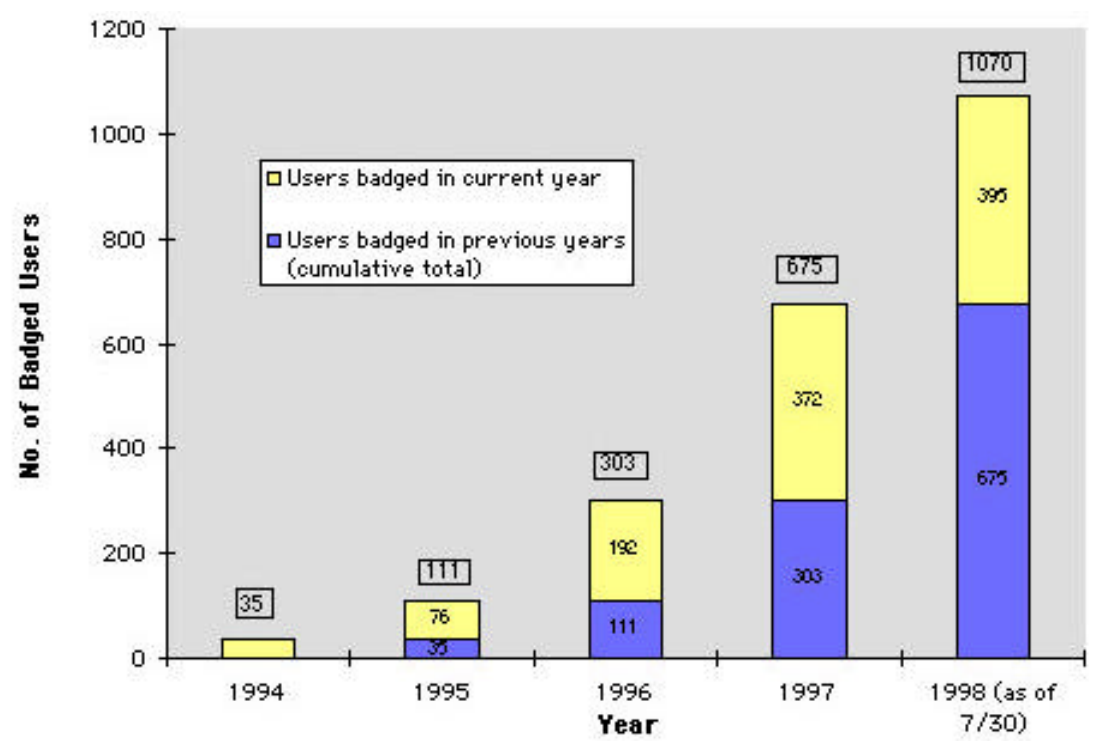

Fig. 3.2 Increase in number of badged APS users by year. 


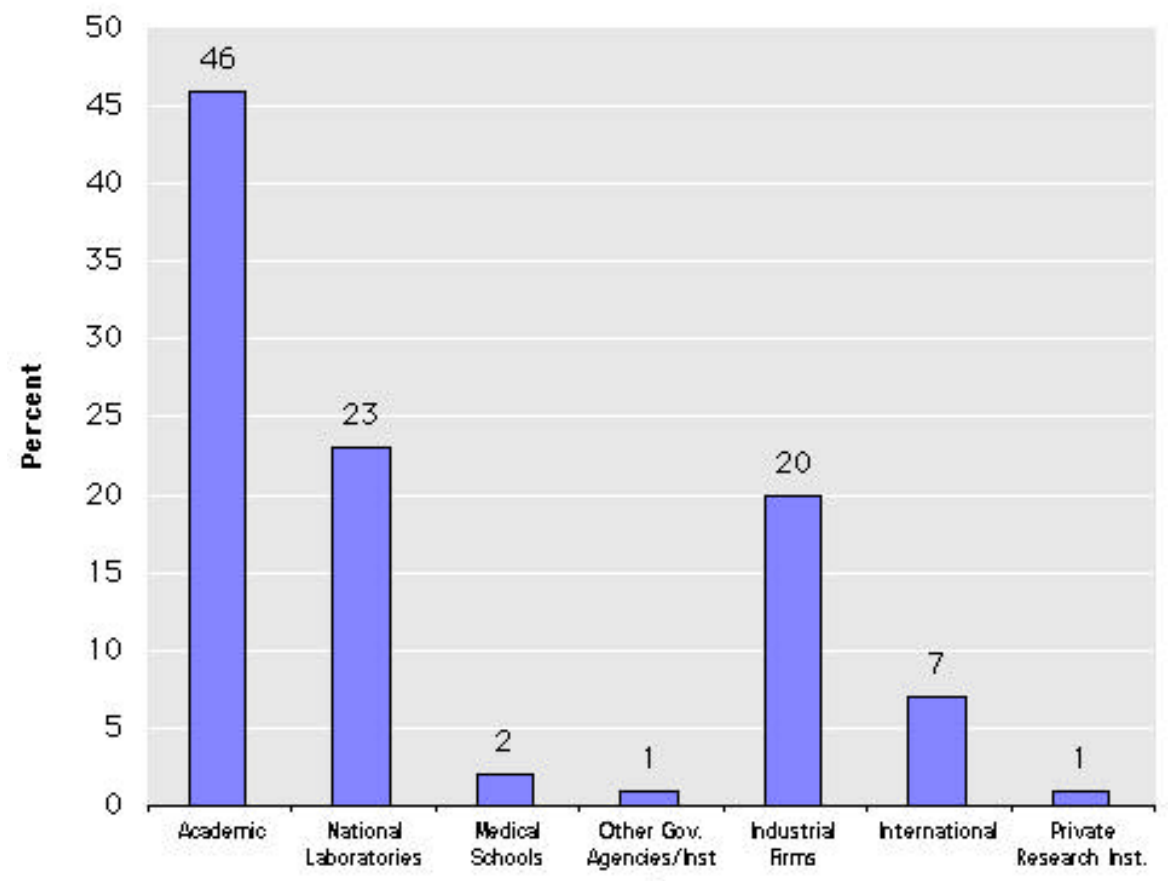

Institutional Affiliation

Fig. 3.3 Percentage of APS users by institutional affiliation (as of 7/30/98).

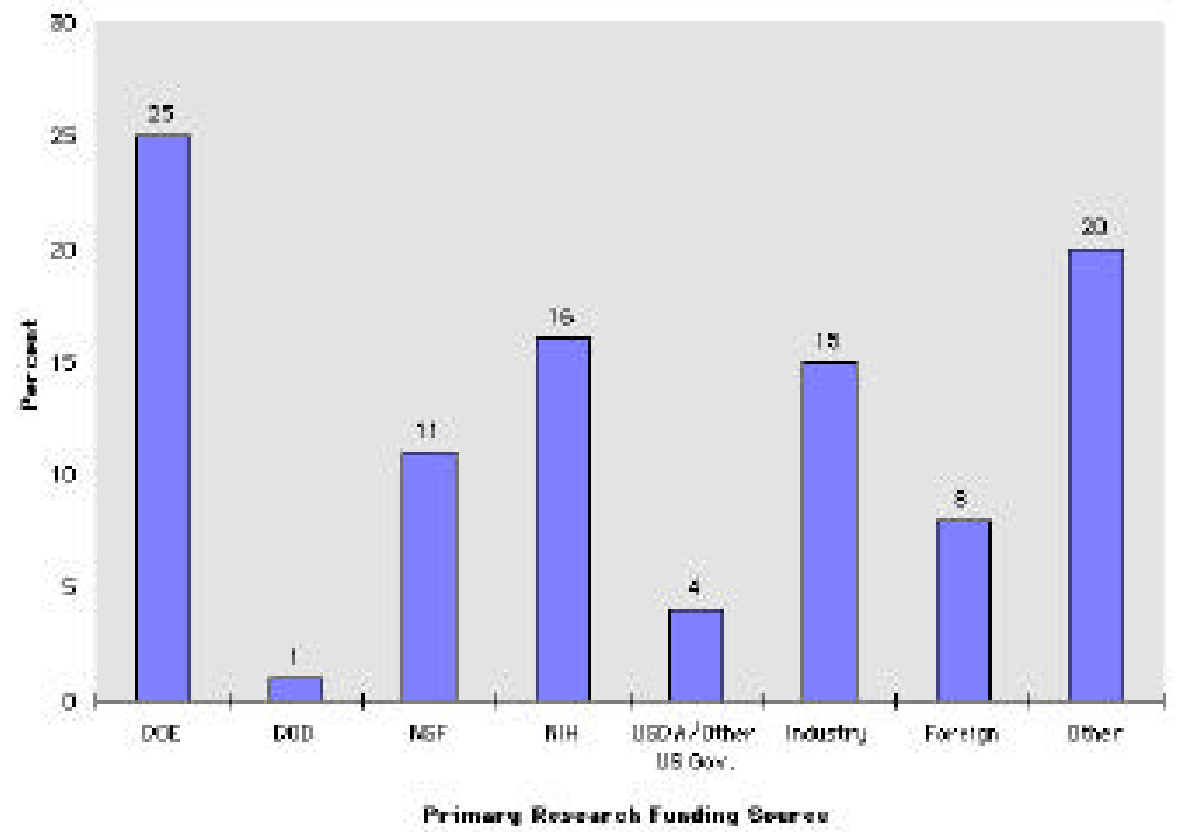

Fig. 3.4 Percentage of APS users by agency that provides research funding (as of 7/30/98). 


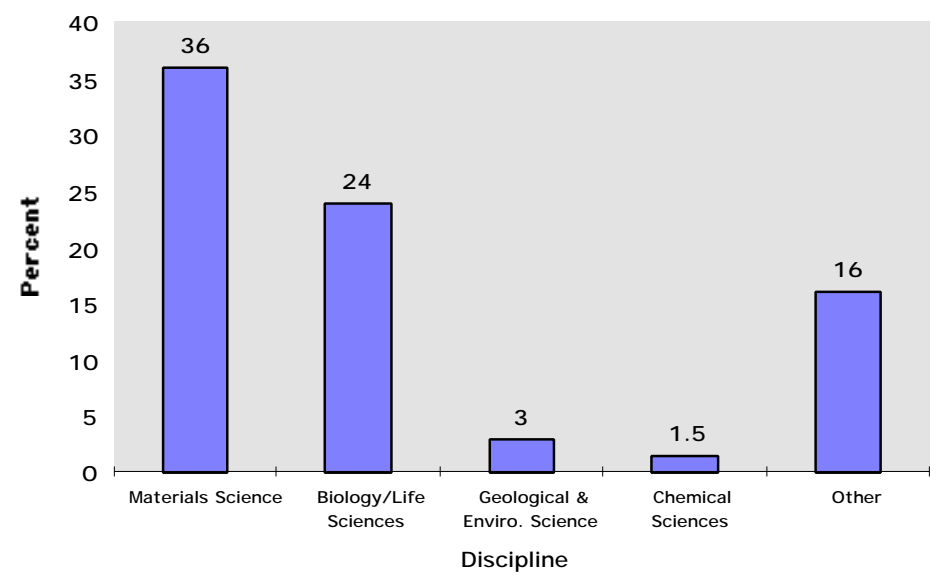

Fig. 3.5 Percentage of APS users by discipline (as of 7/30/98).

\subsection{User Support}

Administrative support for users is coordinated through the APS User Office, which serves as the initial point of contact for new and prospective users. The User Office provides information; registers, orients, and issues badges to new users; manages user data; serves as the APS liaison to the APS Users Organization; provides administrative support to the PEB and Research Directorate (composed of APS top management and the Directors of APS CATs); organizes general meetings and topical workshops for the user community; and handles day-to-day user questions and concerns. User Agreements and Accounts are handled through the Experimental Facilities Division Office, as is user policy development. Beamline design reviews, experiment safety approval forms, and other technical issues are handled by the User Technical Interface Group. Day-to-day support for CATs on the experiment hall floor is provided by the floor coordinators through the Experiment Floor Operations Group (see section 2.8).

\subsubsection{User Communications}

During 1998, a number of vehicles were used to communicate with users: on-site "CAT Chats," the User Information Web page, CAT NET, CAT Communicator, and a new Web site organized and maintained by the APS Users Organization. CAT Chats are held almost every Friday at 3:00 p.m. During this time, APS management and operations staff meet with users in an open forum to discuss issues of common interest. Machine status, scheduling information, upcoming APS and ANL events, and current projects or concerns are all covered in a relaxed, informal setting. Minutes are taken and action items recorded. Status reports on action items are given at the next week's meeting. Archives of CAT Chat minutes are available on the Web. In addition, the User Information Web page contains links to technical and safety-related publications, contact and access information, conference and meeting agendas, operational status reports, CAT Home Pages, and other general information sites. CAT NET, which is an e-mail notification system, is used as needed 
to provide bulletins of interest specifically to on-site users. Two issues of CAT Communicator (a newsletter for APS users with a circulation of approximately 1600) were published during the first half of 1998 . A third issue is planned for distribution in September, and a fourth will be published in December.

\subsubsection{User Registration, Orientation, and Badging}

Before a user can conduct hands-on work at the APS, he or she must register with the User Office and complete both a general orientation course and sector-specific training; in addition, a signed User Agreement must be in place between the APS and the institution sponsoring the work (see below). The general orientation course covers Argonne and APS policies; general safety information such as site alarms, the use of 911 for emergencies, hazard communication, radiation safety, and experiment safety; and the basics of the beamline Personnel Safety System (PSS). Additionally, for unescorted access to the experiment hall floor, a user must complete General Employee Radiation Training (GERT) or prove that he or she has successfully completed GERT at another DOE facility within the previous two years. The general orientation course is now Webbased; although the majority of sessions are conducted in the User Office, some preliminary experiments with distance delivery have been conducted with staff from the University of Florida. GERT is provided in the User Office as computerbased training.
When the orientation course and GERT have been completed, users sign a statement indicating their willingness to comply with ANL/APS policies and guidelines and acknowledging the existence of appropriate User Agreements. Users are then provided with reference copies of the newly revised APS User Guide and the APS User Safety Guide and issued Cardkey® identification badges that enable them to enter both the Argonne campus and the controlled-access APS experiment hall. During 1998, the User Office received permission from the DOE to issue APS user badges, and appropriate badging equipment was purchased and installed. Sector-specific training (required for every sector at which a user works) is the responsibility of the host CAT, which furnishes training records to the User Office for audit purposes. By the end of 1997, a total of 675 users had been registered, oriented, and given badges. Through the end of July 1998, an additional 395 were added.

\subsubsection{User Data Management}

Information about APS users is stored in a relational database system developed by the User Office. The system is flexible and easily modifiable in-house. Current modules include People, Registration, Directory (which functions as a "read-only" electronic "Rolodex" for APS user contact information), CAT Information, II Proposals, User Accounts, User Agreements, User Training, Meetings and Conferences, and the recently added APS Tours. Each module has separate read/write access privileges and is appropriately password protected. The Directory module is 
accessible through the User Information Page on the Web, where individual user entries can be self-updated.

\subsubsection{Support of User Advisory Groups}

Administrative support is provided for three main user-related advisory groups: the 16-member APS Users Organization Steering Committee meets quarterly and serves as a support, advisory, and advocacy group for the APS (the APS User Program Administrator serves as the primary APS liaison for this group); the Research Directorate, which also meets quarterly, is chaired by David Moncton and facilitated by a CAT Director selected by his peers; and the Program Evaluation Board, mentioned earlier, meets at least annually to review new Letters of Intent and proposals and conduct CAT progress reviews. This year, the PEB met for three days in February; a second two-day meeting took place in September.

\subsubsection{Conference and Workshop Organization and Support}

General APS user meetings are held every 18 months, with an average attendance of about 500. Comprehensive organizational support for these meetings is provided by the User Office. The Eighth Users Meeting was held on April 15-17, 1997, and the Ninth Users Meeting, which includes six specialized workshops, is scheduled for October 13-15, 1998. In addition, the User Office provides support as requested for specialized meetings and workshops held at the APS and elsewhere. The most recent was the Sixth International Conference on Biophysics and Synchrotron Radiation held at the APS on August 4-8, 1998, with an attendance of over 300 . The next scheduled meeting with User Office support is the 18th International Conference on X-ray and Inner Shell Processes planned for August 23-27, 1999, in Chicago.

\subsubsection{User Agreements}

As mentioned above, before a user can conduct hands-on work at the APS, a User Agreement must be signed by Argonne National Laboratory and the institution sponsoring the work. These agreements address financial, liability, and intellectual property issues. As of August 7, 1998, APS User Agreements are in place with 140 institutions, including 65 universities and non-profit institutions, 27 industrial firms, 12 U.S. government-funded labs, and 36 international institutions. The list of institutions that have signed APS User Agreements can be found in Appendix 5.

\subsubsection{User Accounts}

User Accounts are established at the APS to enable users to pay for ancillary equipment, supplies, and services, as well as beam time used for proprietary research. Four types of accounts are currently in place: construction, capital equipment, operating, and proprietary. As of July 30, 1998, 105 User Accounts are in place, serving 54 institutions. Of those, 15 are construction accounts, 29 are for capital equipment, 46 for operating expenses, and 15 for proprietary beam time. During the current fiscal year, the funds managed by the APS on behalf of users totaled $\$ 17.1 \mathrm{M}$. 


\subsubsection{User Policies and Procedures}

APS User Policies and Procedures is a comprehensive umbrella document designed to provide guidelines for all aspects of APS participation by CATs and IIs and to clarify the roles of the APS staff and various user advisory groups. Development of user policies and procedures is an ongoing process. A comprehensive index of all polices (both currently existing and under development) appears on the User Information Web page. When new policies are developed (or existing policies are changed), they are presented to the APS Users Organization Steering Committee, the Research Directorate, and the Program Evaluation Board for review and comment. The Associate Laboratory Director for the APS has final approval. As soon as the policy or policy change has been approved, it is added to the Web policy library.

\subsubsection{Beamline Design Reviews}

During the beamline design phase, the User Technical Interface Group (UTIG) works with CATs to ensure that their preliminary and final designs not only comply with the CAT's operational requirements but also comply with applicable safety standards and APS operational requirements. During 199798, Preliminary Design Reports (PDRs) were completed for 18 sectors, and Final Design Reports (FDRs) for 16; additionally, a number of design report updates were reviewed. Installation of beamline systems has continued at a rapid pace throughout the year; this activity is coordinated by UTIG. Continuing support was also provided for the design and installation of user beamline utilities and the construction of user laboratory and office space in the laboratory/office modules (LOMs).

\subsubsection{Technical Policy Support}

Additional efforts this year have been made to tailor APS and ANL requirements to better meet the needs of the growing user community. These activities have ranged from defining the policies and procedures by which users can bring third-party contractors to work in CAT facilities to establishing a standard for transporting small quantities of hazardous materials on the ANL site that is consistent with Department of Transportation requirements. Periodic Technical Updates are used to communicate these developments to the user community.

\subsection{User Safety}

At the APS, safety is a line management responsibility that is shared by the CATs. The following are the basic elements of the approach used by the APS and the CATs to create and sustain a safe working environment for APS users:

- The CATs identify hazards and incorporate appropriate engineered safeguards and procedural controls into their APS facilities and operations.

- Each CAT conducts its activities at the APS in accordance with a written safety plan developed by the CAT and approved by the APS.

- Users receive appropriate safety training for their activities at the APS.

- The APS, ANL, and the CATs themselves perform safety oversight of user activities. 
This overall approach was described in detail in last year's Experimental Facilities Division Progress Report 1996-97 (ANL/APS/TB-30). The Experiment Safety Review process and the Independent CAT Safety Assessment process have continued to evolve since the above report was written; updates on these processes, which are consistent with DOE's guidelines for Integrated Safety Management, are given below. Recent safety actions, reviews, and audit activities are also summarized in this section. A broader summary of XFD's safety activities during FY 1998 is given in Appendix 6.

\subsubsection{Experiment Safety Review}

The CATs have the primary responsibility for safety reviews of proposed experiments. The information needed to perform these reviews is obtained through the use of a standard APS Experiment Safety Approval Form. This form is completed in part by the experimenter, who describes the materials and equipment to be used, the known hazards, and the ways in which these hazards will be mitigated; and in part by the CAT Director or designee, who reviews the information, makes recommendations as needed, and ultimately signs off to indicate approval. An individual designated by the CAT must also sign the form just before the beginning of the experiment to verify that all required safeguards are in place. The form is then posted at the beamline for the duration of the experiment.

A Web-based system for submission and approval of the Experiment Safety Approval form is currently (as of mid-August 1998) in beta testing; it is expected to be available for general use before the end of FY 1998. The system includes safety guidance for the experimenters and the CATs.

An XFD committee oversees the CAT experiment safety review process to ensure compliance with ANL safety requirements and provide additional guidance on safetyrelated issues. The committee (which includes the XFD Division Director, Associate Director for Operations, ES\&H Coordinator, and Experiment Safety Review Coordinator) meets weekly to discuss ongoing and future experiments. The XFD Experiment Safety Review Coordinator serves as the liaison between the CAT Safety Coordinators and the APS.

\subsubsection{Independent CAT Safety Assessments}

To take advantage of the CATs' growing experience in managing their own safety programs at the APS, XFD has also initiated the formation of three Independent CAT Safety Assessment groups, within which the CATs conduct reciprocal assessments of each other's safety programs. A set of model assessment criteria has been provided by XFD. Each of the CATs currently in residence at the APS has named a representative to one of these groups, and the XFD Experiment Safety Review Coordinator is an ex officio member of all three groups. Each CAT is reviewed by the other CATs in its group at least annually, on a rotating basis. After a given CAT is reviewed, it receives a written report (which is copied to XFD) identifying action items and a schedule for completing these actions. The groups are also encouraged to make recommendations to the APS for enhanced safety support. As of mid-August 1998, the safety programs of five CATs have been reviewed in this manner. 


\subsubsection{Safety Actions, Reviews and Audits}

During the past 18 months, ANL has been evaluating its operations for conformance with DOE Policy 450.4, Safety Management Systems Policy. To comply with this policy, DOE contractors' safety programs must reflect seven "Guiding Principles" and define the manner in which five "Core Functions" are being carried out. At the APS, Integrated Safety Management (ISM), the term used to describe the DOE's expectations, is not a new concept.

APS CATs have benefited from the emphasis the APS has long placed on safety planning and the incorporation of CATs into the APS line management structure with regard to safety issues. The general approach the Division has taken in working with the user community is entirely consistent with the ISM principles and functions. For example, the guiding ISM principles can be seen in the "Introduction" to the model CAT safety plan created by XFD. The model plan provides a number of mechanisms for carrying out ISM's five Core Functions. Ongoing reviews and APS oversight activities confirm that the CATs are implementing these mechanisms and that they view safety as a primary management concern.

XFD continues to provide technical and administrative safety support to the CATs through the User Technical Interface Group and the XFD ES\&H Coordinator. The Division also provides ongoing informal surveillance and oversight and periodic audits and reviews. Each of these activities helps CATs to improve their safety performance.
XFD also continues to conduct periodic walk-through inspections of CAT-occupied facilities and to provide feedback to CATS about observed behaviors and workplace conditions. In general, CATs are provided advance notice of these inspections so that knowledgeable CAT personnel will be available to participate and to answer questions posed by the inspection team.

In response to guidance from ANL, XFD conducted a "chemical vulnerability" audit during the last quarter of CY97. The audit found no unacceptable risk or other lack of conformance to expectations. The conclusions of the audit were later validated by a team consisting of representatives from DOE and from ANL's ESH/QA Oversight Group.

Since housekeeping is an important component of safety, XFD organizes a comprehensive cleanup of the experiment floor at least four times a year and more frequent cleanings of the aisleways between sectors. 


\subsection{X-ray Optics Fabrication and Metrology}

The APS users have availed themselves extensively of our capabilities in the areas of metrology, thin film deposition, and crystal fabrication over the past year. A new interferometer for more direct monitoring of a polished surface during the polishing process and an ellipsometer with an in situ capability for the large deposition system have been added to enhance these capabilities with a particular emphasis on achieving smoother surfaces and thinner films. Furthermore, a specialized polisher is being commissioned to further enhance the polishing capabilities available to users, and an atomic force microscope is also being commissioned to enhance the metrology capabilities. Finally, an x-ray reflectometer using a standard tube source for rapid monitoring of multilayer x-ray performance and of layer thicknesses, in order to provide very rapid feedback for deposition parameters, is being commissioned. This instrument will enhance the ability to rapidly achieve a desired multilayer reflecting energy at a given angle of incidence. Progress in side-cooled mirror design for mirror optics (needed with future increases in storage ring current) is also discussed in this section.

\subsubsection{X-ray Optics Metrology Laboratory}

Thanks to fruitful collaborations between the synchrotron radiation community and mirror manufacturers, most of the major mirror vendors have developed their own metrology facilities for evaluation during the fabrication process and for their quality control. However, considering the high cost of a single synchrotron radiation mirror combined with the required delivery time, it is wise, before final acceptance, to independently check the purchased mirror and see if it meets the prescribed specifications. The metrology facility at the APS is designed to help the users fulfill this need. It is equipped with four major instruments, housed in an environmentally controlled Class 100,000 clean room. These instruments include a long trace profilers (LTP), a WYKO-6000 figure interferometer, a WYKO TOPO2D/3D roughness profiler, and an atomic force microscope (AFM) - a TOPOMOTRIX Explorer system. The three first instruments are noncontacting interferometers; the AFM can function in either a contact or a noncontact mode. The LTP is housed in a Class 100 clean enclosure in which the temperature fluctuation is controlled to within $\pm 0.1^{\circ} \mathrm{C}$, in order to minimize system errors. These instruments cover a wide range of spatial frequency and allow one to determine the power spectral density function (PSD). The PSD gives the height distribution of the mirror surface as a function of spatial frequency, which permits one to predict the x-ray performance of the mirror more precisely than considering just figure and finish. Finally, a visible light microscope is also available for visual inspection of optical surfaces.

\section{Some Selected Results}

During the last fiscal year, over 15 major beamline mirrors, as well as several bending mechanisms and numerous small optical substrates, have been characterized at the metrology laboratory of the APS. The mirrors came in a variety of shapes (flat, cylindrical, spherical, and elliptical), with lengths up $1.2 \mathrm{~m}$. Typical mirror substrate 
materials are ULE, Zerodur, and single silicon crystals, with slope errors $\leq 5 \mu \mathrm{rad}$ root mean square (rms), and microroughness $\leq 3 \AA$ rms. Major manufacturers include Bœing, Zeiss (Germany), Seso (France), and Beam Line Technologies.

Most mirrors are for the APS users from BESSRC, Bio, IMCA, and UNI CATs. In addition, the needs of other synchrotron radiation facility users was also supported, including CHESS (one multilayer mirror) and the Advanced Light Source (two LTP spherical reference mirrors).

Also, in addition to intergroup collaborations involving efforts in metrology-fabrication-deposition, the metrology lab has seen an increase in requests for applications other than for synchrotron radiation, mainly from other divisions at ANL. The work includes evaluation of surfaces, such as diamondlike-coated SiC seals (Chemistry Division) and substrates for a mm-wave cavity project (ASD-APS).

\section{Comparison between LTP and X-ray Profiles}

Recently, a comparison made between LTP measurements and those obtained using $\mathrm{x}$-ray diffraction showed the usefulness of the LTP as a rapid and accurate means of evaluating synchrotron radiaton optical surfaces. Such a comparison is illustrated in Fig. 4.1, in which the LTP profile of the SRI CAT 1-BM focusing mirror is compared to that obtained with a synchrotron $\mathrm{x}$-ray beam from the 1-BM source during the commissioning of the mirror. Although the two methods probe different spatial frequencies, the two profiles show almost identical features. The slight discrepancy towards the right edge (see Fig. 4.1) is believed to be largely due to the mirror mounting in combination with the way the data were processed. Also, note that the LTP profile was taken with a 2-mm lateral resolution at 630.8-nm wavelength, while the x-ray scan was obtained with a lateral resolution of approximately $5 \mathrm{~mm}$ at 1.24-nm wavelength (or $10 \mathrm{keV}$ ). As one can see from the figure, the mirror is not perfectly flat; the two humps at the edges are due to the manufacturer's polishing techniques.

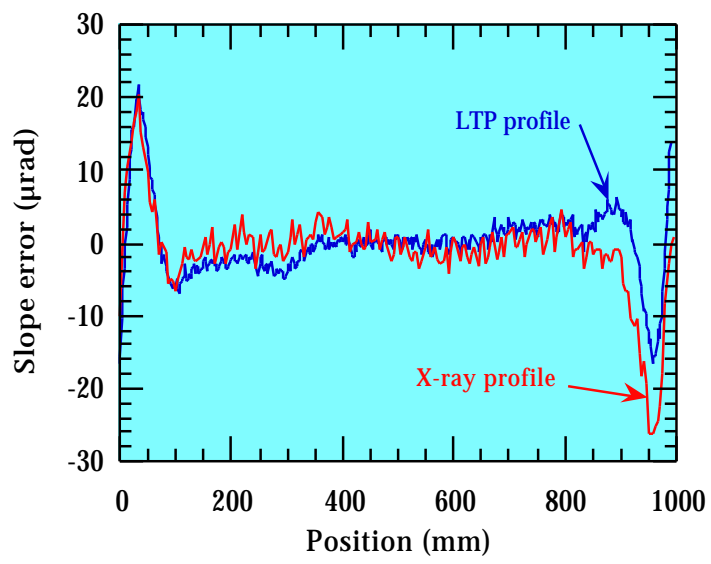

Fig. 4.1 SRI-CAT 1-BM focusing mirror: comparison between the LTP profile (blue curve) and the $x$-ray profile (red curve) measured during the commissioning of the mirror under the $x$-ray beam from 1-BM source (see text for more details). Note that the $x$-ray profile was measured by J. Lang, G. Srajer, and J. Wang of SRI-CAT at the Advanced Photon Source.

\section{An Unusual Case}

To date, almost all measured mirrors are within the users' specifications. The exception was one 800-mm-long flat Zerodur mirror, whose surface showed some 
discontinuities apparently not seen during the manufacturing process. The imperfections revealed by the LTP scans (see Fig. 4.2) were confirmed by subsequent measurements performed using our WYKO6000. Several LTP scans taken across the mirror showed that the observed imperfections are not localized ones but are rather spread all across the width of the mirror.

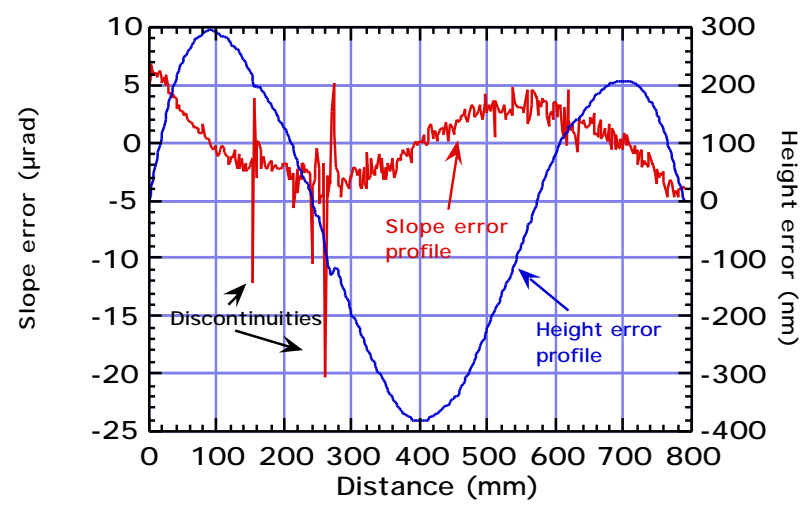

Fig. 4.2 Discontinuities revealed by the LTP profile of an 800-mm-long Zerodur mirror. The detected imperfections were confirmed by measurements performed using our WYKO-6000 profiler.

\section{Improvements to the LTP}

The LTP was mainly designed for measuring mirrors face up. Recently, we extended its capability to evaluating mirrors and benders in three different deflecting configurations: horizontal, vertical, and side ways. We achieved this by adding a modular optical scanning arm to the LTP optical head. The scanning arm is made of a double pentaprism system mounted using commercially available mechanical components. This provides a means of rapidly and accurately calibrating a mirrorbender assembly before installation in the beamline. Fig. 4.3 shows the example of a Bio-CAT mirror-bender assembly under calibration using the described system. The mirror was mounted face down and was held against gravity by a series of tension springs distributed along the mirror length. The mirror's reflecting face was adjusted to be almost perfectly flat after only a few LTP scans, a task that would have required a considerable effort using online $\mathrm{x}$-ray measurements.

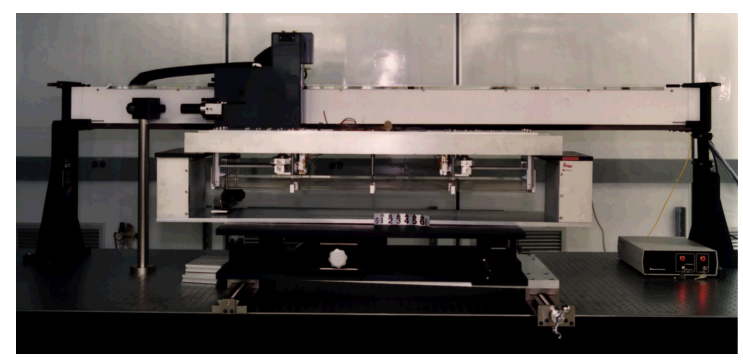

Fig. 4.3 Photograph of a Bio-CAT mirrorbender assembly under the LTP. A double prism scanning arm was added to the LTP optical head in order to be able to scan the mirror face down (i.e., its final configuration). The mirror is held against gravity by a series of tension springs evenly distributed along the mirror length. The surface of the mirror was adjusted to almost a perfect flat in just a few LTP scans, a task that would have required a considerable effort and time using on-line $x$-ray testing.

\section{Other Improvements and Near Future Projects}

Other improvements in the metrology laboratory include: a) connection of the individual computers of these instruments to the APS network, which will make the data accessible for analyses and will provide easy access to results of measurement for users for publication purposes, etc.; and 
b) development of a metrology laboratory Web page.

Near future projects under consideration include: a) acquiring a mechanical stylus profiler to cover a wider range of applications, b) providing the AFM with a stage to accommodate large optics, c) providing the WYKO-6000 figure interferometer with a high-precision stage for evaluating large mirrors at grazing incidence angles, thus complementing the LTP, and d) upgrading the TOPO instrument.

\subsubsection{Deposition Laboratory}

\section{Work Progress}

Our coating facilities are running smoothly and successfully. We have carried out over 100 regular depositions since April 1997. Over 400 mirrors and experimental samples have been made. All deposition requests from users have been completed promptly. $\mathrm{X}$-ray multilayer mirrors, multistrip mirrors, microfocusing mirrors, and $\mathrm{x}$-ray lithography samples have been fabricated using magnetron sputtering. Using houseinvented precision-temperature-controlled evaporators, $\mathrm{Fe}^{57} \mathrm{Sn}^{119}$ alloy samples have been made by co-evaporation.

Increased productivity has been achieved through the design of universal substrate holders. Multiple samples with arbitrary shapes can be loaded into both the large and the small deposition systems without the need to make individual holders. The coating capability in the small deposition system has been expanded to maximum allowable dimensions of 4 " wide, 1.2" high, and 9" in length.

Two 3"-diameter sputter guns have been added in the 1.5-m deposition system. Four different materials can now be coated without breaking the vacuum. RF sputtering for coating insulating materials is also available.

The loading system for the large deposition system has been improved for easy handling of large mirrors. Substrates are loaded inside an air-class 1 clean-hood. Small components and mirrors can be cleaned in the clean-hood before deposition. A UV drying lamp has been installed in the large deposition system to degas the substrates before deposition.

\section{Multilayer Growth and Characterization}

Multilayer x-ray mirrors and experimental samples are routinely fabricated. A 100-bilayer W/C multilayer x-ray mirror made for CHESS showed a high reflectivity of $74.1 \%$ and a low bandwidth of $1.8 \%$. Figure 4.4 shows $\mathrm{x}$-ray reflectivity data for a W/C multilayer.

\section{Ellipsometer for Thin Film and Multilayer Characterization}

A spectroscopic ellipsometer, the M-44 Ellipsometer manufactured by the J. A. Woollam Co., has been added in the deposition lab for both in situ and ex situ thin film characterization. In ellipsometry, a light beam of known polarization is incident on the sample, and the polarization of the 


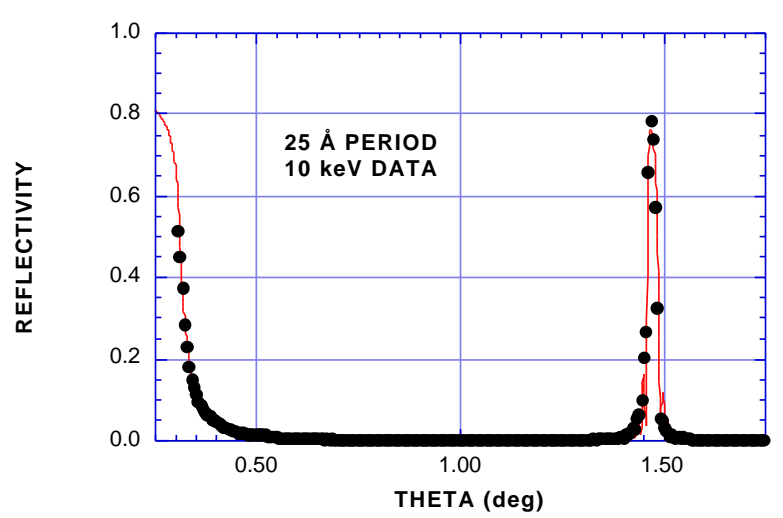

Fig. $4.4 \mathrm{X}$-ray relectivity for a W/C multilayer grown at the APS.

reflected beam is measured and analyzed. The interaction of the beam with the sample causes changes in the polarization of the light. In general, the reflected light is elliptically polarized. Analyzing the shape of this ellipse (hence the term "ellipsometry") can reveal the physical properties of the sample. Usually, ellipsometry is used for dielectrical/semiconducting materials. For metal thin films less than a few tens of nm thick, however, the light will reach the lower interface and the measured quantity will be sensitive to the thickness. Most of our mirror and multilayer coatings fall within this thickness range.

The accuracy of ellipsometry measurements for thin films depends on knowledge of the exact optical constants of the film, which are often not available. Extensive experimentation with different thin film systems has been carried out to meet this challenge. Sputtered thin films with incremental thicknesses were analyzed using in situ ellipsometry with their thickness correlation in mind. We found that the optical constants are thickness dependent for most metal films. Once the optical constants are found for each thickness range, the film thickness can be more accurately measured using ellipsometry.

Ellipsometry can be used to measure multilayer structures once the optical constants are obtained for each component. Figure 4.5 shows the result for a W/C multilayer. Psi $(\Psi)$ and delta $(\Delta)$ are called "ellipsometer parameters." They are related to the ratio of Fresnel reflection coefficients $\mathrm{R}_{\mathrm{p}}$ and $\mathrm{R}_{\mathrm{s}}$ for $\mathrm{p}$ - and s-polarized light as follows: $\mathrm{R}_{\mathrm{p}} / \mathrm{R}_{\mathrm{s}}=\tan (\Psi) \exp (\mathrm{i} \Delta)$. These parameters can be fitted with a regression analysis using the film thicknesses as variables. In the present example, the resulting thicknesses agree with that of the x-ray results within $4 \%$. The ellipsometry experiments demonstrate that good quality control can be expected for our thin film and multilayer coatings.

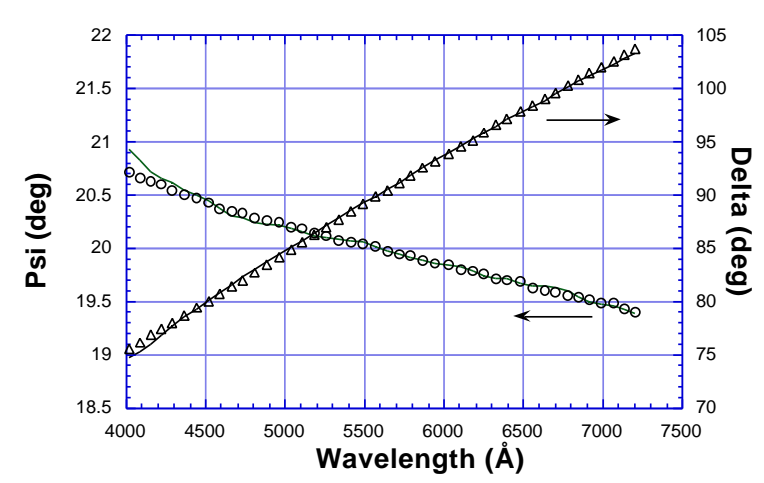

Fig. 4.5 Ellipsometry data and model fit for a $W / C$ multilayer.

\subsubsection{Fabrication Laboratory}

The fabrication laboratory served the APS user community by preparing and/or improving different elements (predominantly crystals) for x-ray beamlines. In total, over the past year, the lab manufactured 120 new crystal elements, improved or modified 12 , and initiated production of 22 others. 
Requests completed included such elements as cryo-monochromators, interferometers and diced analyzers. Improvements and/or modifications of crystals already being used on the beamlines consisted of such operations as reshaping, repolishing and reetching. Forty five percent of orders originated from other than SRI-CAT users, namely from Bio-CAT, BESSRC-CAT, IMM-CAT, PNC-CAT, MU-CAT, MHATT-CAT, UNI-CAT, CARS-CAT and DND-CAT.

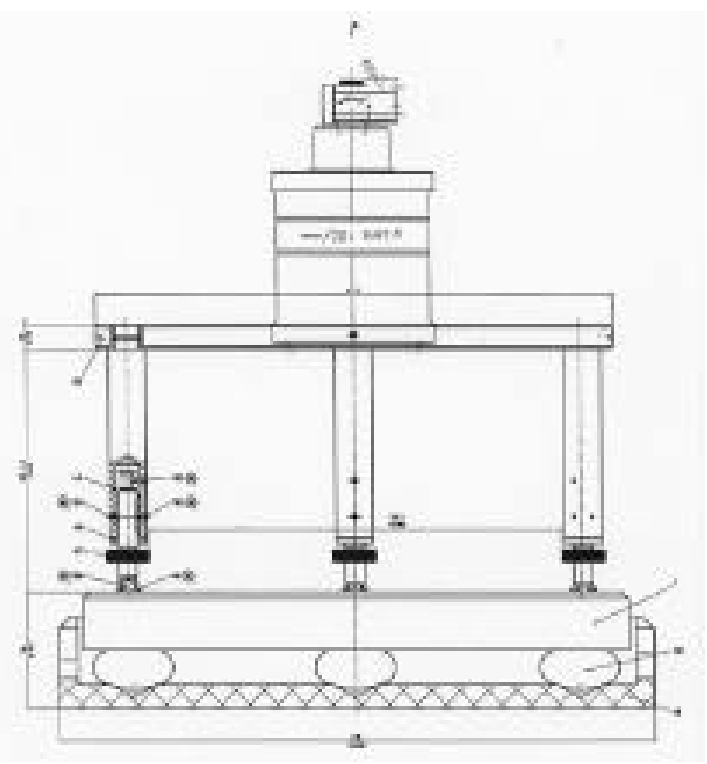

Fig. 4.6 Schematic of the FISBA Optik MicroPhase setup. Shown in the drawing, looking from top to bottom, are a small Twyman-Green-type interferometer, a system of lenses, the base with three legs equipped with regulation screws, a granite base, vibration-isolation pads, and the support box.

The fabrication laboratory is now equipped with its own interferometer. The FISBA Optik MicroPhase setup is a compact modular-type instrument consisting of a small Twyman-Green-type interferometer connected by a fiber guide to a laser (Coherent, Model \#200) and mounted on the top of a system of lenses called a beam extender (see Fig. 4.6). The extender is fixed to a base standing on a vibration-isolated granite plate on three screws. The measured object is placed on the granite plate under the beam extender. Operation of the instrument is controlled by a PC. The system utilizes phase-shifting interferometry and allows one to measure the flatness of optical surfaces of a diameter up to 4". The interferometer measuring range is about $10 \lambda$, and its accuracy is about $\lambda / 10$.

\subsubsection{X-ray Characterization Laboratory}

The x-ray characterization laboratory serves the APS users by giving them the opportunity to orient and test single crystals and to test multi- or single layers deposited on different substrates.

A single-axis diffractometer and a Laue camera were utilized for crystallographic orientation of numerous crystals, predominantly the ones needed in the fabrication laboratory. It is worthwhile to give some examples of the use of other capabilities of the lab. For instance, the double-axis diffractometer placed on a Rigaku x-ray generator was employed to test interferometers that were later investigated with synchrotron radiation, while the left port beam from a Spellman generator was utilized for investigations of archeological samples. However, the main experimental activities in the lab were concentrated at the rotating anode generator.

The Topo Test Unit (TTU) was used for topographic testing of 67 silicon crystals 
(monochromators and analyzers already manufactured, and ingots to be used for fabrication), 12 diamond plates, and 10 crystals of other type (germanium, silicon carbide, sapphire). The majority of samples came from XFD staff, but some tests were done for users from BESSRC-CAT, BioCAT, MHAT-CAT, MU-CAT and IMMCAT. Interesting topography results (see Fig. 4.7) were obtained for pin-post monochromators manufactured for Bio-CAT by Boeing North American, Albuquerque Operations. The measurements revealed patterns of strain present in the diffracting

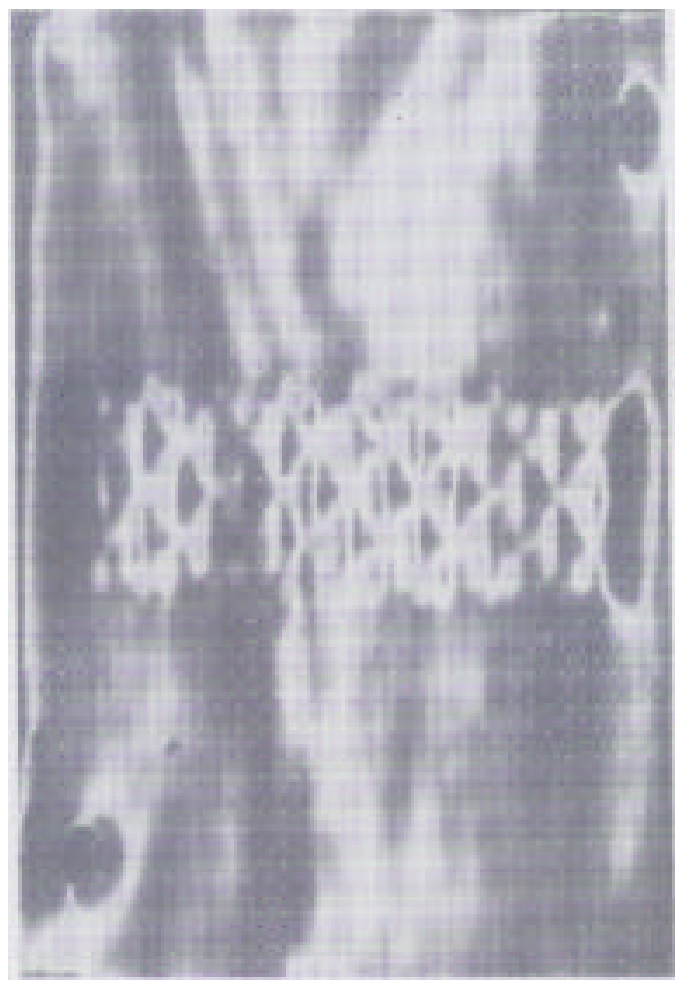

Fig. 4.7 Topograph of the Si (111) pin-post monochromator \#1 revealing a characteristic pattern of strain in the region of the heat exchanger. Silicon reflection (333) and 8-keV photons were used in the experiment. silicon layers that could be attributed to the geometry of the heat exchangers and the bonding technology. The TTU was also used to measure angles between crystallographic and physical surfaces. A series of such measurements was done for Fermi Lab.

The triple-axis diffractometer was used for reflectivity measurements of 26 multi- or single layers deposited on glass or silicon substrates. Most samples were produced in the deposition laboratory. The measurements supplied data needed for deposition of final beamline optics products and/or characterized samples utilized later for synchrotron radiation investigations.

The available equipment in the x-ray lab was enhanced by preparation of some new monochromators and analyzers, e.g., germanium (400) and silicon (620) monochromators for new topographic experiments.

\subsubsection{X-ray Mirror Design and Characterization}

\section{Post-Monochromator Mirrors}

Over the past two years, a large number of $\mathrm{x}$-ray mirrors have been designed and commissioned at the APS. Many of these are positioned downstream of the monochromators and thus received monochromatic beams. Their function is often a combination of focusing, harmonic suppression, and beamline branching. Most of these mirrors are evaluated for surface quality or are coated (single and multilayer) here at the APS. The coatings are characterized using both optical (visible) and $\mathrm{x}$-ray techniques. 
For rapid characterization of mirrors and single- and multilayer-coated substrates (e.g., evaluation of coating quality and thickness for establishing deposition rate in the deposition laboratory), a laboratory $\mathrm{x}$-ray system consisting of a Spellman $x$-ray source and a suitable beam guide has been designed and is being assembled. X-rays are produced by a conventional $\mathrm{x}$-ray tube $(\mathrm{Cu}$ or Mo targets) with a maximum power of 2 $\mathrm{kW}$. A horizontal beam from this source is directed, consecutively, through a slit, a monochromator, another slit, a beam scatterer (to determine photon counts by a bicron scintillation detector), and through another slit onto the mirror being analyzed. The mirror is placed on a Bede stage that is designed to fit onto a $\theta-2 \theta$ goniometer, and the two in combination provide the necessary smooth rotation and translation of the sample for detailed mirror surface analyses. The reflected beam from the substrate passes through a slit and an analyzer to a Bede detector, which has over 5 orders-of-magnitude linear dynamic range. The stage, goniometer, and several of the slits are motorized for rapid sample evaluation. Standard APS beamline data acquisition hardware and software are used with a friendly computer interface on a Sun workstation. Design of this system is completed, and hardware are ready and in final stage of assembly. Additionally, a compatible but modular setup at the APS sector 2 bending magnet beamline is planned so that samples evaluated in the lab can also be easily tested on a beamline.

\section{Mirrors as First Optical Elements}

In addition to the post-monochromator mirrors, an increasing number of APS beamlines (at least 6) use or plan to use a mirror as the first optical element. This approach to beamline design has been motivated, at least in part, by the success in the design of simple high-heat-load mirrors at the APS and by their reliability and ease of operation established over the past two years. As an example, the contact-cooled high-heat-load mirror at the sector 2 undulator beamline of the APS has been operating for two years without any mirror-related downtime. The preliminary thermal and structural performance of this mirror at commissioning and during subsequent observations indicates a tangential rms slope error of about 2-4 $\mu \mathrm{rad}$ (Khounsary et al., 1998); a precise measurement of the mirror has been planned for this summer (1998).

Plans have also been made for precise reevaluation of this mirror using 'pink' beam radiation this summer. For this, we have designed a cooled composite slit array consisting of a thin copper plate with thirty holes, each $60 \mu \mathrm{m}$ in diameter and located at $300-\mu \mathrm{m}$ center-to-center intervals, masking another slit array of thirty $30-\mu \mathrm{m}$ holes configured in a thin tantalum plate. These two plates are aligned, with the one having larger holes acting as a thermal shield for the second plate. Pink undulator beam reflected from the mirror passes through the slit array and is recorded on a charge-coupled device (CCD). Transient and steady-state tangential slope errors in the mirror under various heat load conditions are mapped from the shifts in the slit images on the CCD camera.

Another attribute of the mirror-first beamline design is the ability to use a watercooled monochromator downstream of the mirror. A simple contact-cooled monochromator-dubbed U-monochromator for its U-shaped cross section-was designed, installed, and evaluated. Both early evaluations (Lee et al., 1997) and recent experience indicate that this contact- 
cooled monochromator is capable of comfortably handling post-mirror undulator beam.

\section{Mirror Characterization Using an in situ LTP}

Another set of measurements aimed at evaluating mirror performance under highheat-load x-ray beam was made using an in situ long trace profiler developed at the APS sector 2-ID beamline in collaboration with Brookhaven National Laboratory. In this setup, a laser beam scans the length of a contact-cooled high-heat-load 200-mm-long mirror to determine its transient and steadystate slope errors. Preliminary results (Takacs and Randall, 1998) indicate a thermally induced slope error of about $7 \mu \mathrm{rad}$ at steady-state conditions and a thermal time constant of about 10 minutes. These are in excellent agreement with design predications made earlier (Khounsary and Yun, 1996).

\section{X-ray Mirror Design for Higher Beam Powers}

As new generations of vacuum chambers make smaller undulator gap openings possible and as enhancement in storage ring capabilities make ring operation at higher beam currents possible, the ramifications of using mirrors as first optical elements with substantially higher power x-ray beams need to be examined. A program aimed at developing mirrors for higher heat loads has led to a design that is expected to meet the requirements.

With closer undulator gap and/or higher storage beam current, the heat load on mirrors used as the first optical element will be substantially increased. At a gap opening of $11.5 \mathrm{~mm}$, a typical 1.2-m-long mirror located at about $30 \mathrm{~m}$ from the source at a $0.15^{\circ}$ angle with respect to the $\mathrm{x}$-ray beam (reflecting photons up to $35 \mathrm{keV}$ with a $\mathrm{Pt}$ coating) receives about $1.2 \mathrm{~kW}$ of power and a peak heat load of about $0.4 \mathrm{~W} / \mathrm{mm}^{2}$. The maximum temperature and rms slope errors are $45^{\circ} \mathrm{C}$ and $2 \mu \mathrm{rad}$, respectively. At a closer 10.5-mm gap and a hypothetical 300-mA storage ring current, the incident heat load on the same mirror will be $5 \mathrm{~kW}$ with a peak heat flux of $1.5 \mathrm{~W} / \mathrm{mm}^{2}$. The increased heat load lead to higher temperature, higher slope errors, and higher stress in the mirror. While moderate heat load increases (up to about 30\%) on the present mirrors can be tolerated at the cost of about $50 \%$ increase in slope error, more significant increases can lead to unacceptable temperature, slope errors, and stresses.

We have embarked on designing another simple contact-cooled mirror aimed at handling, with acceptable performance, the above hypothetical heat load. The key features of this mirror design are (1) introduction of a pair of notches in the mirror substrate (see Fig. 4.8) for a more effective thermal moment balance in the substrate, (2) replacement of the indium foil used as interstitial material (between the copper cooling and silicon mirror) with In/Ga eutectic for a more efficient heat transfer, and (3) an increase in the cooling block width to reduce substrate temperature. Preliminary analyses indicate that incorporation of these three features would lead to a mirror with under 5- $\mu$ rad slope error with a maximum temperature of about $80^{\circ} \mathrm{C}$ for the above hypothetical power load. Stress levels in the mirror are high, and a prototype should be made and tested under simulated heat-load conditions. 


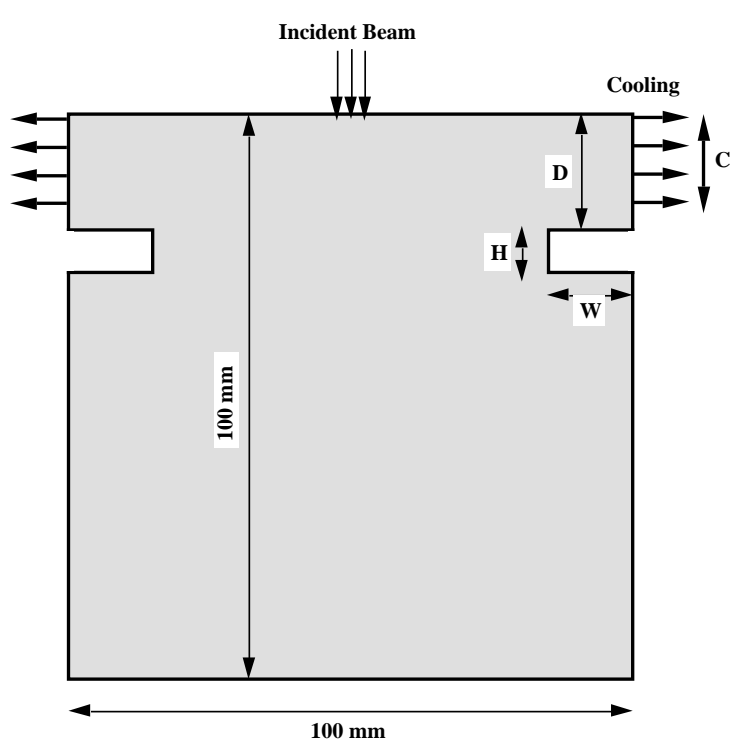

Fig. 4.8 The cross section of a 1.2-m-long mirror showing a pair of notches that help establish a thermal profile in the substrate, which restores thermal moment balance keeping the mirror tangentially (into the paper) close to flat. With an incident undulator A beam power of over $5 \mathrm{~kW}$, a slope error under 5 urad is expected.

\section{Beryllium Window Evaluation and Characterization}

Another issue related to mirrors-whether high heat load or not-is the surface quality and its impact on x-ray beams. Most x-ray mirrors installed at the APS have very fine figure and finish, typically in the range of 2-5 microradians and 2-4 $\AA$, respectively. There is some questions as to whether speckles seen in some x-ray images are related to the mirror surface quality or the beryllium $(\mathrm{Be})$ windows used on these beamlines. To that end, the APS has initiated a collaborative effort with the Be window manufacturer, Brush Wellman, to determine whether the observed speckles resulting in beam quality degradation are due to the Be windows or to the mirror surface, and if, as believed, the Be window is the culprit, the APS will develop guidelines for window surface in order to reduce or eliminate the observed beam quality degradation.

To determine the effect of $\mathrm{Be}$ windows on beam quality, in collaboration with Brush Wellman, a pair of Be windows have been designed for installation on an APS beamline. A number of Be foils of varying surface roughness are made and are being evaluated at the APS, using both optical (visible) and x-ray techniques. For the first time, very thin (250- $\mu$ m-thick) Be foils have been polished to very fine finish (as low as $100 \AA \mathrm{rms}$ ) on both sides and from various Be grades (cold-rolled and bulk). After these are characterized, the smooth foils are brazed to the cooled window frame to provide a pair of smooth, cooled Be windows for installation on an APS beamline. Residual beam degradation, if any, should then be due to x-ray mirrors. This work is in progress.

\section{Phosphor Coating for CCD Detector}

One aspect of the CCD detector development at the APS involves phosphor coating of the fiber optic substrates for high efficiency and resolution. A new in-house coating technique is being developed to replace the existing but often complex coating methods that produce poor quality coatings with significant residual chemical waste. The method consists of controlled spin-coating of the phosphor, prepared in a suitable solution, onto substrates producing very smooth coatings. Another goal of this work is to develop and evaluate fiber optic substrates with a layer of phosphor and an additional antireflection film directly 
deposited on them to make simple, integrated substrates for use in $\mathrm{x}$-ray detectors.

\subsection{Beamline Controls and Data Acquisition}

The XFD beamline-controls effort has two complementary missions of approximately equal priority: (1) to develop generalpurpose control and data-acquisition software that all APS CATs can use and provide technical support to CAT developers; (2) to implement software and computer-related infrastructure (network, file server, workstations, printers, etc.) for the SRI-CAT beamlines and laboratories. This arrangement implies that SRI-CAT users test software for the rest of the facility and provide much of the feedback directing its development. Our contact with other APS users is generally indirect, through their developers.

Nearly all APS beamlines run XFDdeveloped software, though the implementations vary from CAT to CAT. Some CATs run virtually the same software as does SRI-CAT, from user interface through low-level drivers; others run our low-level and middle-level software in their VME crates and supplement or replace our user-interface layer with their own custom software. The APS Beamline Controls Collaboration, which includes developers representing all APS CATs, chose EPICS as the basis for beamline software development in part because it allows for this kind of flexibility of implementation.

EPICS does not guarantee this flexibility, however; nor does the mere implementation of support for a device (e.g., an optical table) or a technique (e.g., on-the-fly scanning) in EPICS software guarantee that the support can be used facility-wide. Aside from the quality of a software module, the layer in which it is implemented determines whether it can be run by higher-level software and whether it can run lower-level software. Software that can do both provides the greatest flexibility, and consequently much of our focus is on this kind of software.

Most of XFD's beamline-software development effort is in server-side software-the low-level and middle-level software that runs in VME crates-as opposed to client-side software that runs mostly on workstations. Roughly half of our development is of driver software, which interacts directly with hardware and clearly must run on the server side. But much of the rest could be done either in server or in client software, and we choose server-side development whenever possible-sometimes even when client-side would be a more natural choice-because anything implemented in server software can be used by all CATs, regardless of their choices of client software, workstation type, and operating system.

The past year's development effort has focused on five general areas: improvements in scan and data-storage software; support for message-based devices; support for remote beamline operation; support for new devices; and exploratory studies of software, hardware, and techniques that may one day become useful in beamline control. 


\subsubsection{Improvements in Scan Software}

The EPICS scan software described in last year's report worked around a fundamentally limited completion detector-the mechanism for determining when positioners or detectors have completed their movements or acquisition. The old completion detector could be fooled by network latency into thinking that a set of positioners was done if the "busy" and "done" messages from one positioner were received before the "busy" message from another was received. The completion detector also required developers to maintain a list of all slow devices eligible for scans. This list had to be modifiable at run time, and the implementation of that requirement limited scan speeds to a few tens of data points per second. Finally, the completion detector was confusing to new users and a frequent source of trouble.

The new scan software takes advantage of recent improvements in EPICS that allow it to track the flow of execution through a complex assembly of software modules linked together at run time, and to report completion of the entire assembly to the module that initiated the operation. We modified the scan software to use this new capability as a completion detector. Most of our custom records, databases, and sequence programs also required modification, to follow the new rules that allow this completion detector to function properly.

The resulting system allows users to scan any correctly implemented device without identifying it in advance to the scan software, is unaffected by normal network latency, and is nearly an order of magnitude faster than the old system. Scans can now acquire several hundred data points per second, significantly extending the range beyond which custom hardware (e.g., waveform generators and multichannel scalers) is required. One type of run-time coordination supported by the old completion detector is no longer supported directly, although users sophisticated enough to require that type of coordination can program the new scan software to run the old completion detector as a pseudo positioner.

\subsubsection{Improvements in Data-Storage and Display Software}

The data-storage and display software described in last year's report consisted of a single client program running on a workstation. That arrangement was expedient and got us through a few difficult years, but it put storage and display priorities in conflict, and resulted in a complex event loop that made the software difficult to maintain end extend. Also, the dual-use program allowed users to delete online data, and one user did this unintentionally.

Now, data storage is handled by a client running in the VME crate that writes directly to the file server. Normally priviledged users can no longer delete raw data, and data storage continues through workstation reboots, license-manager restarts, etc.

Display software for online data is now handled by a separate program with no responsibilities other than display. The program is still somewhat complicated because it must monitor real-time data using EPICS calls and read recently stored data from data files. (For scans of two or more 
dimensions, both real-time and stored data must be displayed.)

For long-term data storage, and for exporting data to users' home facilities, the plan is still to use the Hierarchical Data Format (HDF) based file format agreed on by the APS Beamline Controls Collaboration and supported by third party browsers and some data-analysis packages. This format, now called NeXus, has evolved significantly in recent years, and the performance of HDF has improved as well, though we still cannot write NeXus files fast enough to keep up with real-time data acquisition.

\subsubsection{Support for Message-Based Devices}

Visiting APS users occasionally bring special-purpose serial or GPIB devices with them and need to integrate support for these devices into the control system. CARS-CAT developed generic modules to send and receive strings to/from serial and GPIB ports, but users still have to format and parse the strings. Until recently, that task required custom client software, and the resulting device support could not be used by serverside software. We filled part of the need with boot-time configurable modules that server software could call, but the result was unsatisfactory, arcane, and difficult to maintain.

This year we bridged the gap by adding string support to EPICS run-time programmable calculation software (with which many users already are familiar). Now run-time programmable support for message-based devices behaves just like native EPICS device-support software, is useable both by client-side and by serverside software, and can easily be programmed by users. This means we can fully integrate selected functions of most serial or GPIB devices into the control system in a few minutes.

\subsubsection{Support for Remote Beamline Operation}

EPICS has always provided for shared local/remote beamline control. The capability has thus far been used mostly by developers for technical support and by a small number of users to do simple things like checking the progress of very longrunning scans from home.

This year we found a user for serious remote beamline operation. We wrote software to manipulate a remotely controllable video camera using an unmodified Web browser, modified some public-domain software to send selected video streams (also to an unmodified browser), installed standard EPICS client software on a workstation at the University of Florida, and demonstrated running a beamline from there to an audience of developers, users, and program directors.

\subsubsection{Other Highlights}

Over the past year, we responded to roughly 2300 tech-support requests. This number is significantly lower than last year's, and the requests are qualitatively much different. We received relatively few requests for hardware information, since most of that information is now on our Web pages. Most of the CAT developers are well up the EPICS learning curve by now, and our Web- 
based software documentation is also much improved. The requests we do get generally are more complex than in previous years and, on average, take much longer to handle.

We performed system and network administration for six beamlines and related labs. The control systems supported by our group now comprise 27 VME crates, approximately 60 computers, and 12 printers.

We hosted the second international NOBUGS (New Opportunities for Better User Group Software) workshop for software developers from synchrotronradiation and neutron-scattering facilities. Of 106 participants, 49 were from Argonne, including 31 from the APS.

We have largely taken over EPICS builds for the APS project and distribution of most EPICS software for the EPICS collaboration. We also have begun contributing to the maintenance of Hideos software and VxWorks board-support software on both of which most APS beamline-control systems depend.

In addition, we have:

- Developed support for two new optical-table geometries and now allow for fewer than six degrees of freedom

- Delivered 110 motor-signal interface boards to CATs, bringing the total to 300

- Converted all SRI-CAT workstations from the SunOS operating system to Solaris
- Solved the input-termination problem in the StepPack motor-driver interface board and fabricated sufficient patch kits for all APS beamlines

- Developed hardware and software support for servo motors, piezodrivers, and the Keithley 2000 scanning digital multimeter

\subsection{References}

Khounsary, A. M., W. Yun, B. Lai, I. McNulty, Z. Cai (1998) "Performance of side-cooled high-heat load mirrors," paper presented at the SPIE Conference on Advances in Mirror Technology for X-ray, Synchrotron, and Laser Applications Conference, San Diego, July 20, 1998.

Khounsary, A. M. and W. Yun (1996) Rev. Sci. Instrum. 67 (9) CD ROM.

Lee, W-K., P.B. Fernandez, A. Khounsary, W. Yun, and E. Trakhtenberg (1997) SPIE Proc. Vol. 3151, 208-215.

Takacs, P. and K. Randall (1998) "Mirror distortion measurements with an in situ LTP," paper presented at the SPIE Conference on Advances in Mirror Technology for X-ray, Synchrotron, and Laser Applications Conference, San Diego, July 20, 1998. 


\subsection{Radiation Source Performance}

\subsubsection{Undulator A Performance}

Twenty-two insertion devices are now installed in the storage ring (Table 5.1). During 1998, we acquired considerable operating experience with these devices. The first measurements made on undulator $A$ at the APS were reported previously (Cai et al., 1996; Ilinski et al., 1996). This year, additional diagnostics and analyses were performed on the sector 8 undulator (UA2).

The UA2 undulator is a standard $3.3-\mathrm{cm}-$ period APS undulator A (Dejus et al., 1994), which was positioned downstream from the center of the straight section at sector 8 . The diagnostics included the angular-spectral measurements of the undulator radiation to determine the undulator radiation absolute spectral flux and the particle beam divergence. The undulator diagnostics setup included a pinhole slit assembly to reduce the incident power and to define the angular acceptance and a crystal spectrometer for absolute spectral flux measurements (Ilinski et al., 1996). All components were mounted on a standard APS optical table with five

Table 5.1. Insertion devices installed at the APS as of August 1998.

\begin{tabular}{|l|c|}
\hline \multicolumn{1}{|c|}{ Type } & Number \\
\hline 3.3-cm-period undulator (undulator A) & 17 \\
\hline 5.5-cm-period undulator & 1 \\
\hline 2.7-cm-period undulator & 1 \\
\hline 1.8-cm-period undulator & 1 \\
\hline 8.5-cm-period wiggler & 1 \\
\hline elliptical multipole wiggler & 1 \\
\hline
\end{tabular}

degrees of freedom, so that the setup axis could be adjusted to the axis of the undulator radiation and transverse scans could be performed. The setup was installed in the first optical enclosure (FOE) after two $250 \mu \mathrm{m}$ Be windows.

The results of the undulator UA2 diagnostics showed that the undulator spectrum measured at different gaps (18.0, 18.5, $19.0 \mathrm{~mm}$ ) is close to what one could expect. At a gap of $18.5 \mathrm{~mm}$, the measured undulator flux is in a good agreement with the calculated flux (as shown in Fig. 5.1). Flux calculations were performed using the measured undulator UA2 magnetic field profile at a gap of $18.5 \mathrm{~mm}$ and the values of the particle beam divergence, particle energy spread, and distance to the source. Comparisons were done for the standard storage ring lattice and for the lattice with low vertical beta function.

For the standard lattice that was used in $1997\left(\beta_{\mathrm{x}}=14.2 \mathrm{~m}, \beta_{\mathrm{y}}=10.0 \mathrm{~m}\right.$, coupling $=$ $2-4 \%$ ), the measured absolute flux for the first and third harmonics at a gap of $18.5 \mathrm{~mm}$ was within $1 \%$ of the calculated undulator flux. The measured flux of the fifth harmonic was $15 \%$ less than calculated. For the low-beta lattice that has been used since February $1998\left(\beta_{\mathrm{x}}=16.6 \mathrm{~m}, \beta_{\mathrm{y}}=\right.$ $3.0 \mathrm{~m}$, coupling $=1.2-1.7 \%$ ), on-axis flux density of the first harmonic decreased to $92 \%$ of that for the standard lattice configuration. The flux density did not change significantly because the horizontal divergence dominates the vertical when twodimensional convolution is performed. Nevertheless, the larger vertical divergence for the low-beta lattice configuration could require increasing the size of the vertical aperture of the apparatus to obtain the same total photon flux as for the standard lattice configuration. 


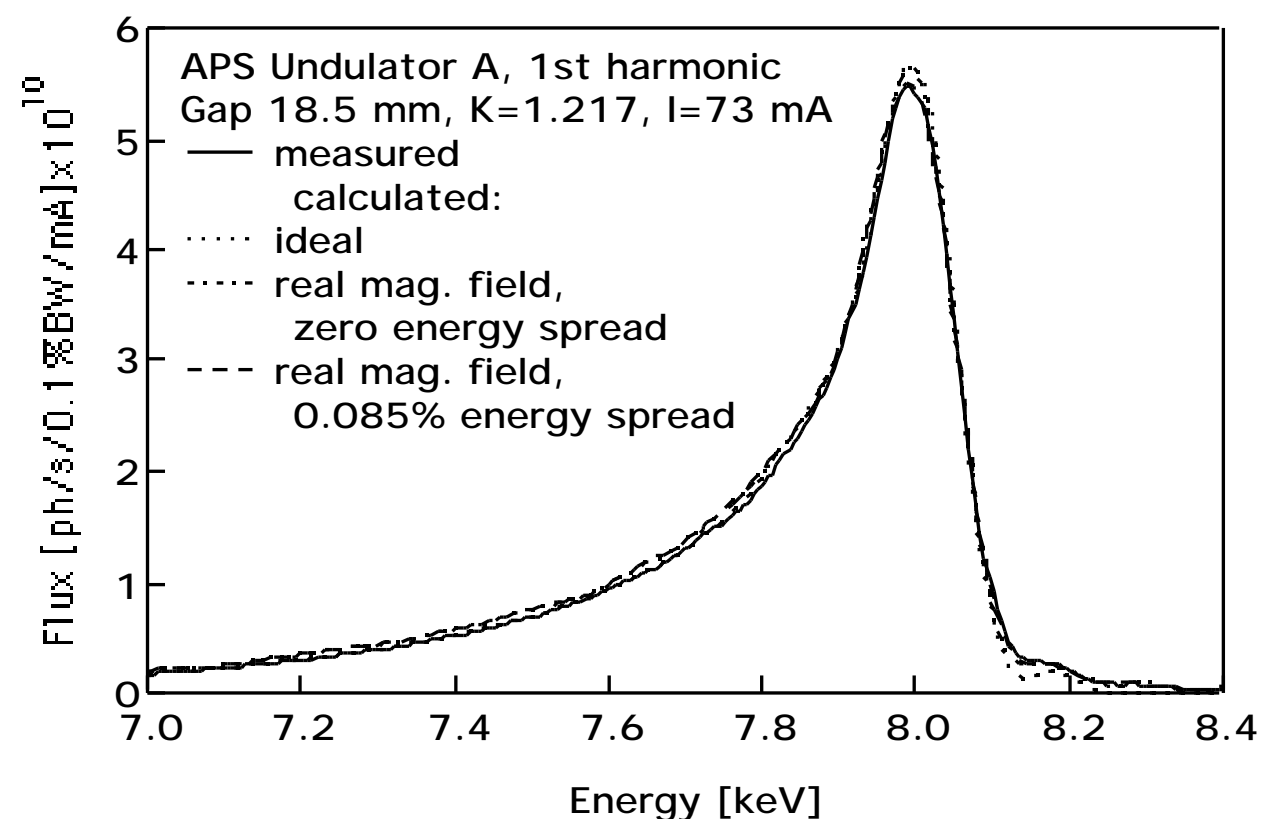

Fig. 5.1 Undulator first harmonic absolute flux at a gap of $18.5 \mathrm{~mm}$. Measured (solid), calculated through the $150 \times 75 \mu \mathrm{m}$ aperture at $28.0 \mathrm{~m}$ for $\sigma_{h}=300 \mu \mathrm{m}, \sigma_{h^{\prime}}=24.0$ $\mu \mathrm{rad}$, for $\sigma_{v}=50 \mu \mathrm{m}, \sigma_{v}^{\prime}=3.9 \mu \mathrm{rad}$ : ideal (dotted line), measured magnetic field and zero energy spread (dashed-dot line), measured magnetic field and $0.085 \%$ energy spread (dashed line).

Beam divergence was determined from the measured undulator transverse profiles at the detuned harmonic energy, which is less than the fundamental harmonic energy. Measuring angular distribution at the detuned harmonic energy allows one to improve the accuracy of the beam divergence evaluation, because the beam divergence dominates over the intrinsic divergence of the undulator radiation. However, the observed asymmetry of the undulator transverse profiles at the detuned energy may complicate the analysis. From comparison of the profile asymmetries for different storage ring lattice configurations, the assumption was made that the asymmetry effect is due to the nature of the particle beam as opposed to being a property of the undulator.
We found that the undulator second harmonic profile is more sensitive to the horizontal beam divergence changes, and the odd harmonic profiles to the vertical beam divergence. The rms particle beam divergences of $24.0 \pm 0.9 \mu \mathrm{rad}$ (horizontal) and $3.9 \pm 0.3 \mu \mathrm{rad}$ (vertical) were determined for the standard storage ring lattice configuration, and $22.5 \pm 0.9 \quad \mu \mathrm{rad}$ (horizontal) and 5.9 $\pm 0.3 \mu \mathrm{rad}$ (vertical) for the low-beta configuration. When beam divergence is determined from the transverse profiles, the main source of the systematic error is the inaccuracy of the beam size.

A particle energy spread of $0.085 \%$ was determined from fitting the calculated width of the third harmonic to the measured one. For a $0.085 \%$ particle energy spread, the 
harmonic widths of the calculated first and fifth harmonics do not exactly match the measured widths of the undulator harmonics. The calculated full width half maximum (FWHM) of the fifth harmonic is $330 \mathrm{eV}$ compared to $366 \mathrm{eV}$ for the measured value.

The diagnostics of undulator $\mathrm{A}$ at sector 8 demonstrated once more that the performance of the standard APS ID is quite close to that of an ideal undulator. Also, readily available ID diagnostics equipment with capabilities to measure spectral and spatial radiation distributions proved to be an essential tool at this user facility.

\subsubsection{Elliptical Multipole Wiggler Performance}

Over the past decade, synchrotron radiation has increasingly been used to probe the magnetic properties of materials. These measurements have generally involved the modulation (or analysis) of the polarization of the incident (or scattered/absorbed) x-ray beams. Techniques, such as circular magnetic X-ray dichroism, core level photoemission, and magnetic Compton scattering, which utilize circularly polarized photons, have attracted particular interest. Because circularly polarized photons couple differently with the magnetic moment of an atom than do neutrons, they are able to provide unique magnetic information not generally accessible by neutron techniques. The development of circularly polarized x-ray diffraction and spectroscopy techniques, however, has been hampered by the lack of efficient sources. Measurements thus far have been primarily taken utilizing off-axis synchrotron radiation from a bending magnet source, which greatly limits incident x-ray flux. The available flux is particularly important for these types of experiments, due to the inherently small nature of the magnetic $\mathrm{x}$-ray cross section. To increase the available circularly polarized x-ray flux, an elliptical wiggler was first proposed and built by Yamamoto et al. (1989). This device consisted of a series of dipole magnets supplemented by a horizontal magnetic field for tilting particle trajectories up and down to obtain circularly polarized radiation along the axis of the wiggler.

Efficient sources of circularly polarized $\mathrm{x}$-rays should provide both the highest possible flux and degree of circular polarization $\left(\mathrm{P}_{\mathrm{c}}\right)$. Recently, an elliptical multipole wiggler (EMW) has been designed, built, and operated on the 11-ID beamline of the APS to provide just such characteristics. A comparison of the EMW with other techniques used for the production of circularly polarized x-rays has been given (Lang et al., 1996). The EMW provides a helicity-switchable $(<10 \mathrm{~Hz})$, highly polarized, high flux source of hard $\mathrm{x}$-rays $(5<\mathrm{E}<200 \mathrm{keV})$. The first measurements characterizing the performance of this device between 10 and $100 \mathrm{keV}$ have been performed. A detailed description of the EMW facility at the APS has been previously reported (Montano et al., 1995), and only a brief description will be given here. The EMW is based on a design by Gluskin et al. (1995); it has 16 periods and a period length of $16 \mathrm{~cm}$. The 24-mm wiggler gap yields a horizontal deflection parameter $\left(\mathrm{K}_{\mathrm{y}}\right)$ of 14.6, corresponding to a critical energy of $32 \mathrm{keV}$ and a total wiggler power of $5.4 \mathrm{~kW}$ at $100 \mathrm{~mA}$. An electromagnet controls the vertical deflection parameter $\left(\mathrm{K}_{\mathrm{X}}\right)$, which can be continuously varied from 0.0 to 1.3 . The use of the electromagnet allows for rapid helicity reversal up to $10 \mathrm{~Hz}$. The 
performance of the EMW was verified by measuring the absolute value of the spectral flux, as well as the degree of linear and circular polarization. The absolute flux and degree of linear polarization measurements were performed by using scattering from a gas in combination with an energydispersive detector (Fig. 5.2). This method has been used previously to characterize the spectral flux of undulators (Ilinski et al., 1995; Hahn et al., 1997; and Cai et al., 1996) A direct confirmation that the spectral flux was circularly polarized was made by performing magnetic Compton scattering measurements on an iron sample (Fig. 5.2).

In Fig. 5.3, the measured on-axis flux from this device for two values of $\mathrm{K}_{\mathrm{X}}$ is plotted as a function of photon energy and compared with calculations. The comparison is very satisfactory. The degree of circular polarization $\left(\mathrm{P}_{\mathrm{C}}\right)$ is obtained for different values of $\mathrm{K}_{\mathrm{x}}$ using two independent methods. In the first, the horizontal $\left(\mathrm{I}_{\|}\right)$and vertical $\left(\mathrm{I}_{\perp}\right)$ radiation components were measured using the gas-scattering technique for values of $\mathrm{K}_{\mathrm{x}}=0.8,1.0$, and $1.3, \mathrm{~K}_{\mathrm{y}}=$ 14.4 , and over the energy range from $10 \mathrm{keV}$ to $100 \mathrm{keV}$. From these measurements, the degree of linear polarization is derived using $\mathrm{P}_{\mathrm{L}}=\left(\mathrm{I}_{\|}-\mathrm{I}_{\perp}\right) /\left(\mathrm{I}_{\|}+\mathrm{I}_{\perp}\right)$. Assuming that there is no unpolarized radiation contributing to these measurements, $P_{X}=\sqrt{ }\left[1-P_{L}^{2}\right]$. In Fig. 5.4, the value of $P_{C}$ obtained this way is compared with the calculations for an ideal particle trajectory through this magnetic device. The differences are well outside the rms error of the measurement $(<5 \%)$ and can only be ascribed to the assumption that

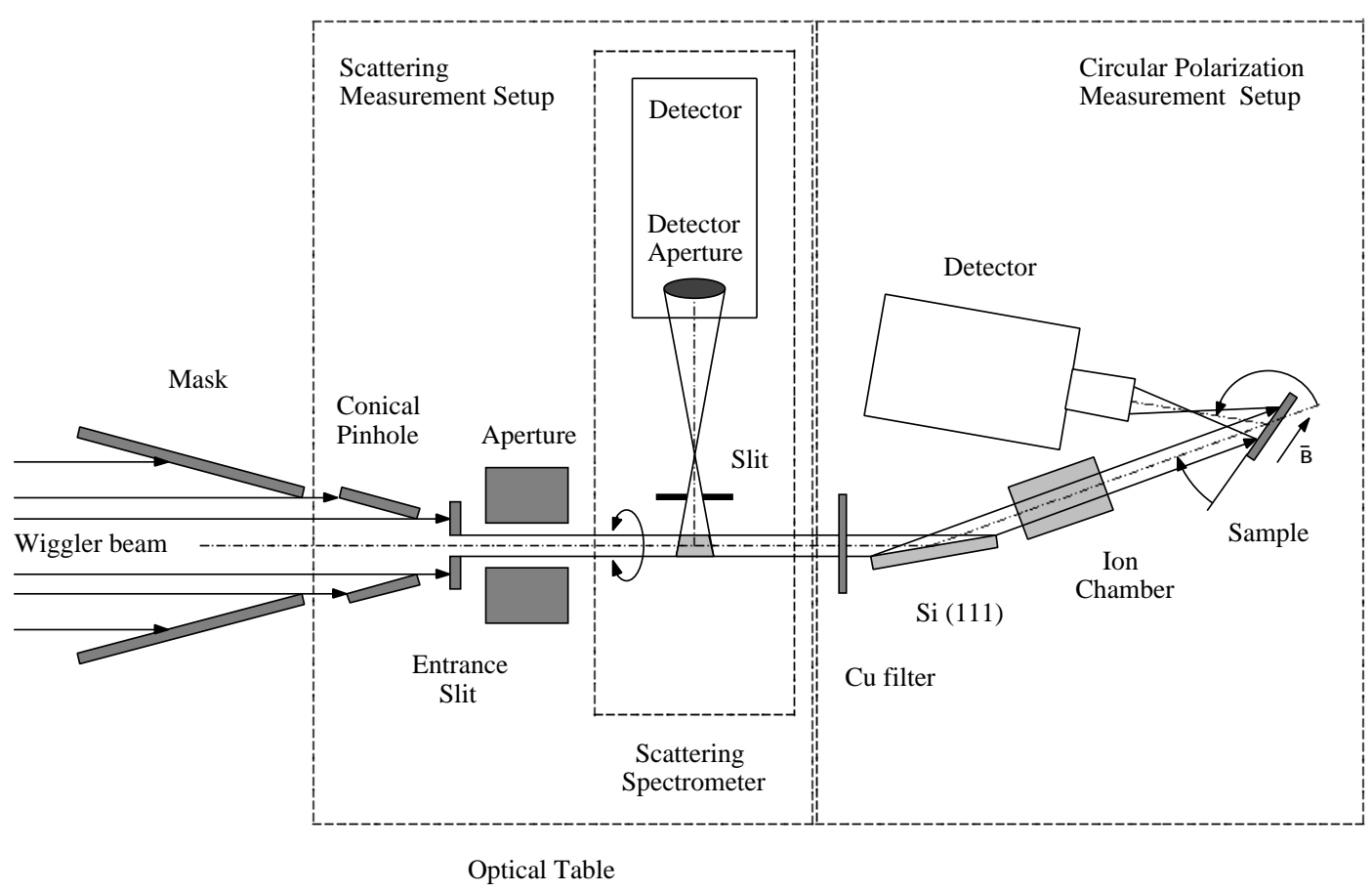

Fig. 52 Side view of the experimental setup used for the characterization of circularly polarized radiation from an EMW. 


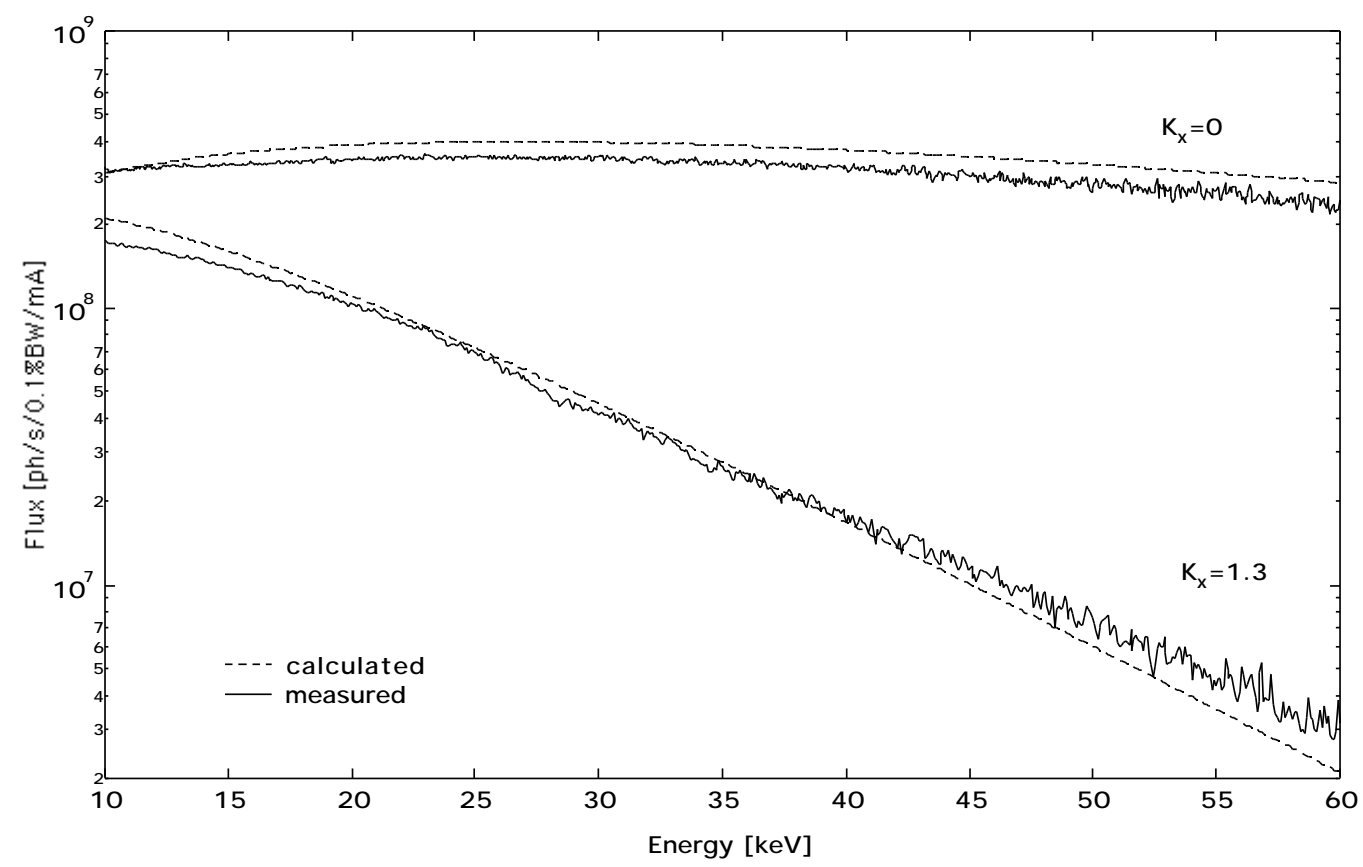

Fig. 5.3 Calculated (dashed) and measured (solid) absolute flux vs. photon energy for $K_{x}=0$ and $K_{x}=1.3$ through the $75(v) \times 150(\mathrm{~h}) \mu \mathrm{m}$ aperture at $28.75 \mathrm{~m}$. The value of $K_{y}=14.4$ for the EMW.

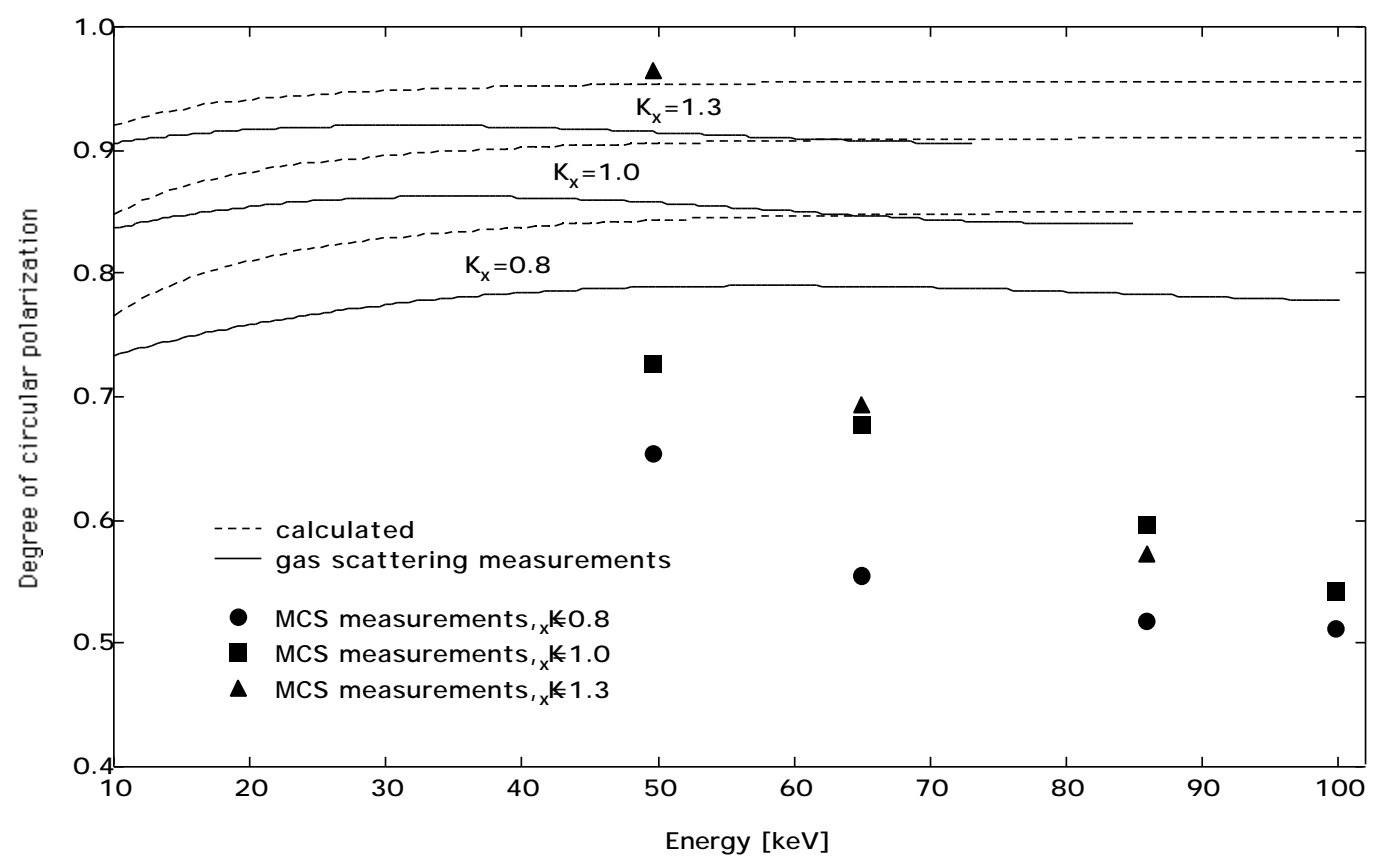

Fig. 5.4 Degree of circular polarization $\left(P_{c}\right)$ vs. photon energy for $K_{x}$ values of 0.8 , 1.0, and 1.3. The value of $K_{y}=14.4$ for the EMW. 
there was no contribution from unpolarized radiation. A second method was devised in which the $\mathrm{P}_{\mathrm{C}}$ is directly measured using magnetic Compton scattering (MCS). The measurements are shown in Fig. 5.4. These measurements do not agree with the results from the gas-scattering measurements or the calculations, except at the lowest energy of measurement. The principal reason behind this discrepancy is attributed to the differing beamline component geometries used in the two methods. In the flux intensive method involving MCS, the beamline apertures were larger, diluting the circularly polarized radiation with linearly polarized radiation. In addition, the apertures used were more transparent at higher energies, increasing the contribution from the linearly polarized photons and thus decreasing the value of $\mathrm{P}_{\mathrm{C}}$ at higher photon energies. A detailed discussion on this and other systematic errors in this measurement are given by Ilinski et al. (1997).

While this device is extremely useful, especially at high x-ray energy, in magnetic studies great caution must be exercised in choosing only the central cone of radiation to maximize the degree of polarization. The device is now used regularly by BESSRC CAT users in sector 11.

\subsubsection{Circularly Polarized Undulator (CPU)}

Both linearly and circularly polarized x-rays have been very successfully applied to the study of magnetic properties of materials. However the applications have been limited primarily due to the lack of energy-tunable, high-brilliance $\mathrm{x}$-ray beams with adjustable polarization properties. Optics (e.g., quarter wave plates) can be fabricated to simultaneously provide a high-quality beam with adjustable polarization in the energy range above $5 \mathrm{keV}$ starting with a linearly polarized beam from a standard undulator A source. Such optical schemes are harder to implement below $5 \mathrm{keV}$. To cover this lowenergy regime, we are developing, in collaboration with the Budker Institute (Novosibirsk, Russia), a helical undulator that can generate beams of variable (linear or circular) polarization. This undulator is a new type of fully electromagnetic device with the ability to choose either horizontal or vertical plane-polarized or elliptically polarized radiation. The device with a $12.8-\mathrm{cm}$ period is based on a new design that uses both horizontal and vertical electromagnetic poles. The poles are constructed in a $\mathrm{C}$ geometry, which allows the insertion device to be installed with a standard APS vacuum chamber (Fig. 5.5). The first harmonic of this device will cover the energy range from $0.4 \mathrm{keV}$ to $3.5 \mathrm{keV}$. (See Table 5.2 for a list of parameters.) An important and unique feature of a fully electromagnetic device is that it will allow us to generate $100 \%$ horizontally $\left(\mathrm{K}_{\mathrm{X}}=0\right)$ or vertically $\left(\mathrm{K}_{\mathrm{y}}=0\right)$ plane-polarized radiation, which will enable many experiments to be performed that otherwise would not be technically feasible. With symmetric deflection parameters $\left(\mathrm{K}_{\mathrm{x}}=\mathrm{K}_{\mathrm{y}}\right)$, the on-axis radiation will be $\sim 100 \%$ circularly polarized. Figure 5.6 shows the brilliance range of the CPU in linear polarization mode, together with that of a 5.0-cm-period elliptical device planned for the Advanced Light Source (ALS EPU). The details on the location and 


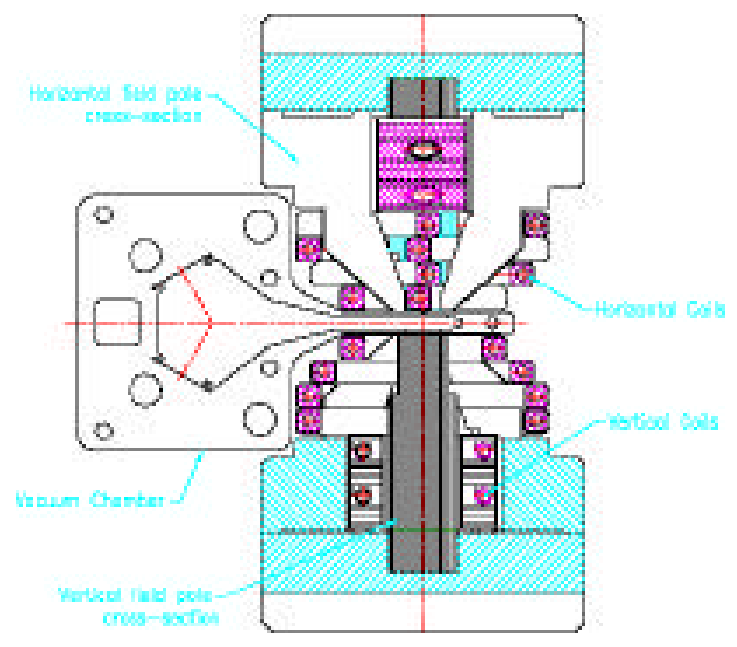

Fig. 5.5 Cross section of the CPU poles nesting around the ID vacuum chamber showing the horizontal and vertical coils.

Table 5.2. Parameters of the 12.8-cmperiod intermediate energy $\mathrm{CPU}$.

\begin{tabular}{|l|l|}
\hline \multicolumn{1}{|c|}{ Circularly Polarized Undulator } & \\
\hline Period & $12.8 \mathrm{~cm}$ \\
\hline Number of full vertical poles & 35 \\
\hline Number of full horizontal poles & 36 \\
\hline Overall length & $2.4 \mathrm{~m}$ \\
\hline Vertical pole gap & $11 \mathrm{~mm}$ \\
\hline Maximum magnetic field & $0.24 \mathrm{Tesla}$ \\
\hline Energy range & $0.5-3.0 \mathrm{keV}$ \\
\hline Switching frequency & $0-10 \mathrm{~Hz}$ \\
\hline $\begin{array}{l}\text { Switching rise time (including } \\
\text { overshoot) }\end{array}$ & $<20 \mathrm{~ms}$ \\
\hline Electromagnet dc stability & $<1 \%$ \\
\hline Maximum total power & $800 \mathrm{~W}$ \\
\hline Maximum power density & $17 \mathrm{~W} / \mathrm{mm}^{2}$ \\
\hline
\end{tabular}

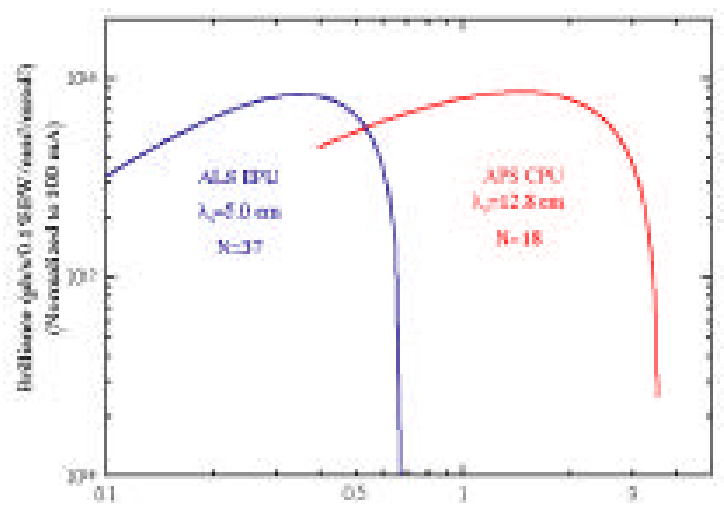

Fig. 5.6 Comparison of helical undulator brilliance. 
installation of the CPU are discussed in the next section.

\subsubsection{Simultaneous Operation of Two Undulators}

At the APS, the straight sections in the storage ring have a length of $5.0 \mathrm{~m}$, thus permitting the installation of two undulators of maximum length $2.4 \mathrm{~m}$. Such configurations of two undulators can enhance the capabilities of the APS. Two unique configurations have been planned. In the first, two 2.4-m-long undulators A will be installed on the straight section in sector 1. The beam, with nearly double the power (and power density), will permit the evaluation of the ultimate performance of nitrogen-cooled Si optics and water-cooled diamond optics. This R\&D will support user operations at stored beam currents much higher than $100 \mathrm{~mA}$.

In the second configuration, two undulators with dissimilar periods will be installed on the same straight section to derive two independent radiation beams with different spectral properties. The proposed installation on sector 4 will have undulators with periods of $3.3 \mathrm{~cm}$ and $12.8 \mathrm{~cm}$, providing radiation above and below $5 \mathrm{keV}$ and covering the energy range from 0.5 to $50 \mathrm{keV}$.

The sector 4 beamline will be the first at the APS designed explicitly to operate with two undulators simultaneously. By inserting a weak horizontal steering magnet between the two tandem IDs, a deflection or 'dogleg' can be produced in the particle beam orbit. This will cause the radiation from the two undulators to be horizontally separated on the experimental hall floor. The hard $\mathrm{x}$-ray range will be covered using the APS standard undulator A. A custom-built 12.8-cm-period variably polarized undulator (see previous section) will be used for the intermediate energy x-ray range. An 8-mm beam separation in the FOE at approximately 30 meters from the center of the straight section is desired. This will require a dipole electromagnet sufficient to steer the beam through 270 microradians. Three permanent magnet dipoles will be installed: one in front of the undulators, one between the undulators, and one after the undulators to create the dogleg.

\subsubsection{5-mm Chamber and Undulator Measurements at 8.5-mm Gap}

In January 1998, a 5-meter-long 5-mmaperture ID vacuum chamber was installed in sector 3 to allow the 2.7-cm-period undulator to achieve smaller gaps. To enable operation of the storage ring without sacrificing lifetime, a low-beta lattice $\left(\beta_{\mathrm{x}}=\right.$ $16.6 \mathrm{~m}, \beta_{\mathrm{y}}=3.0 \mathrm{~m}$ ) tuning was developed by ASD. The 5-mm-aperture vacuum chamber allowed the minimum undulator gap to be decreased to $8.5 \mathrm{~mm}$. This extended the x-ray energy range and filled in gaps in the tuning curve, as shown in Fig. 5.7, where the dotted sections of the lines show the additional capability allowed by the smaller minimum gap. Experimental measurements were made of the photon output at a variety of energies, and the measurements agreed well with calculated predictions.

This device delivers higher brilliance than the 3.3-cm-period undulator $\mathrm{A}$ in the energy range above $10 \mathrm{keV}$. The availability of the 5-mm-aperture vacuum chamber will enhance the capability of the user scientific 


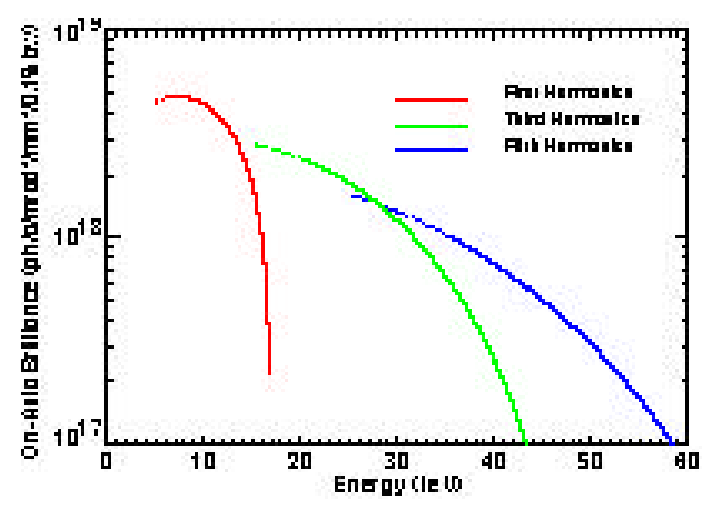

Fig. 5.7 Tuning curves for the 2.7-cmperiod undulator. The dotted line extensions of the curves show the increase in tuning range achieved by decreasing the minimum gap to $8.5 \mathrm{~mm}$.

programs at the APS. Many of the CATs have expressed interest in using the small aperture chamber, which will impact two distinct scientific areas: (1) deriving photon energies down to $2.0 \mathrm{keV}$ using the existing 3.3-cm-period undulator A (which is of special interest to the biological and environmental research communities to reach the absorption edges of elements such as sulphur), and (2) deriving high-brilliance photons at higher energies using the $2.7-\mathrm{cm}-$ period undulator in conjunction with the small aperture vacuum chamber. The impact will be primarily in condensed matter and materials science research, which require both high brilliance (or coherence) at higher photon energies (in comparison with the performance of the 3.3-cm-period undulator).

These new capabilities will impose higher power-handling requirements on the first optics in the beamlines, an area of continuing R\&D in XFD (see section 5.3).

\subsubsection{Storage Ring Installation - Phase-2}

Vacuum chambers for IDs have been installed in 21 sectors of the storage ring; 35 sectors are available for ID beamlines. In 1998, efforts began to fabricate most of the components necessary to complete the ID instrumentation for the remainder of the storage ring. Ten additional 5-meter-long 8-mm-aperture vacuum chambers are being fabricated, and eleven undulator A type $(3.3 \mathrm{~cm}$ period) magnetic structures were procured from STI Optronics for delivery beginning in December of 1998. The CPU (described in section 5.1.3) will be installed in sector 4. Electrical racks and power distribution have been added to the storage ring tunnel roof to accommodate the installation of IDs in the remaining sectors.

\section{New Gap Separation Mechanism}

A new design for an ID gap separation mechanism has been completed and procurement of components to assemble a prototype is underway. The goal of the new design is to improve ID gap positioning accuracy while reducing mechanical complexity to reduce cost. The design uses four drive motors, one for each end of each jaw, to move each end of the top and bottom jaws independently-thereby simplifying the mechanical drive trains while allowing fine adjustments to the parallelism of the jaws. The load bearing frame is assembled from stress-relieved welded structural steel beams (Fig. 5.8). The system has been designed to be compatible with the existing undulator A magnetic assemblies and to allow a minimum gap under load of $10 \mathrm{~mm}$. 


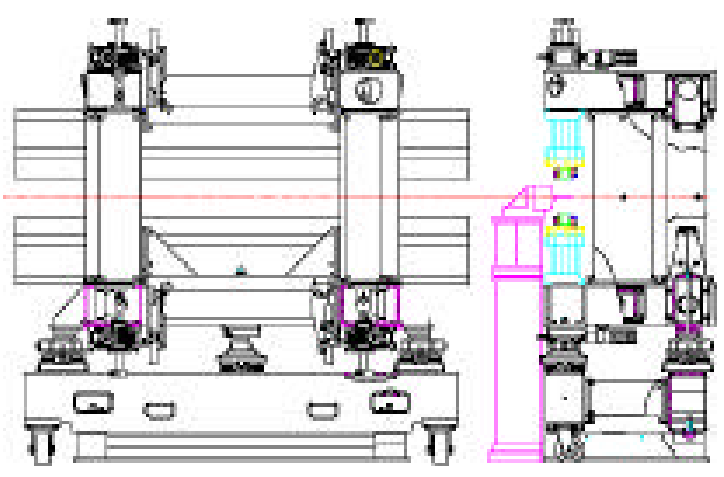

Fig. 5.8 New gap separation mechanism for undulator $A$.

The frame is extremely rigid; finite element calculations show a deflection of less than $3 \mu \mathrm{m}$ when the $3.3-\mathrm{cm}$-period magnetic structure is closed to an $8.5-\mathrm{mm}$ gap.

\subsubsection{Collaborations}

\section{BESSY Chambers}

For several years, the ID group of XFD has collaborated with the staff of the Berliner Elektronenspeicherring-Gesellschaft fur Synchrotronstrahlung (BESSY), located in Berlin, Germany, to design and fabricate ID vacuum chambers for the BESSY II project. These chambers used the innovative technology developed by the APS for small aperture vacuum chambers. In 1998 this project was completed; ten chambers were delivered to Berlin for installation into the storage ring. One of these chambers is shown in Fig. 5.9.

\section{DESY Chambers}

The Deutsches Elektronen-Synchrotron (DESY), located in Hamburg, Germany, is building a vacuum ultraviolet (VUV) FEL based on the TESLA Test Facility linear accelerator. The APS is an official partner in this project. Towards this objective, XFD is designing and fabricating the small-aperture extruded-aluminum vacuum chambers that will be used for the FEL undulators. Seven 9.5-mm-aperture chambers, each $4.516 \mathrm{~m}$ long, are being built. Figure 5.10 shows the first chamber after preliminary machining. The project is expected to be completed in the spring of 1999, and the FEL tests will be performed during the fall of 1999. The FEL operated at $1 \mathrm{GeV}$ is expected to produce radiation with a wavelength of $70 \mathrm{~nm}$. 


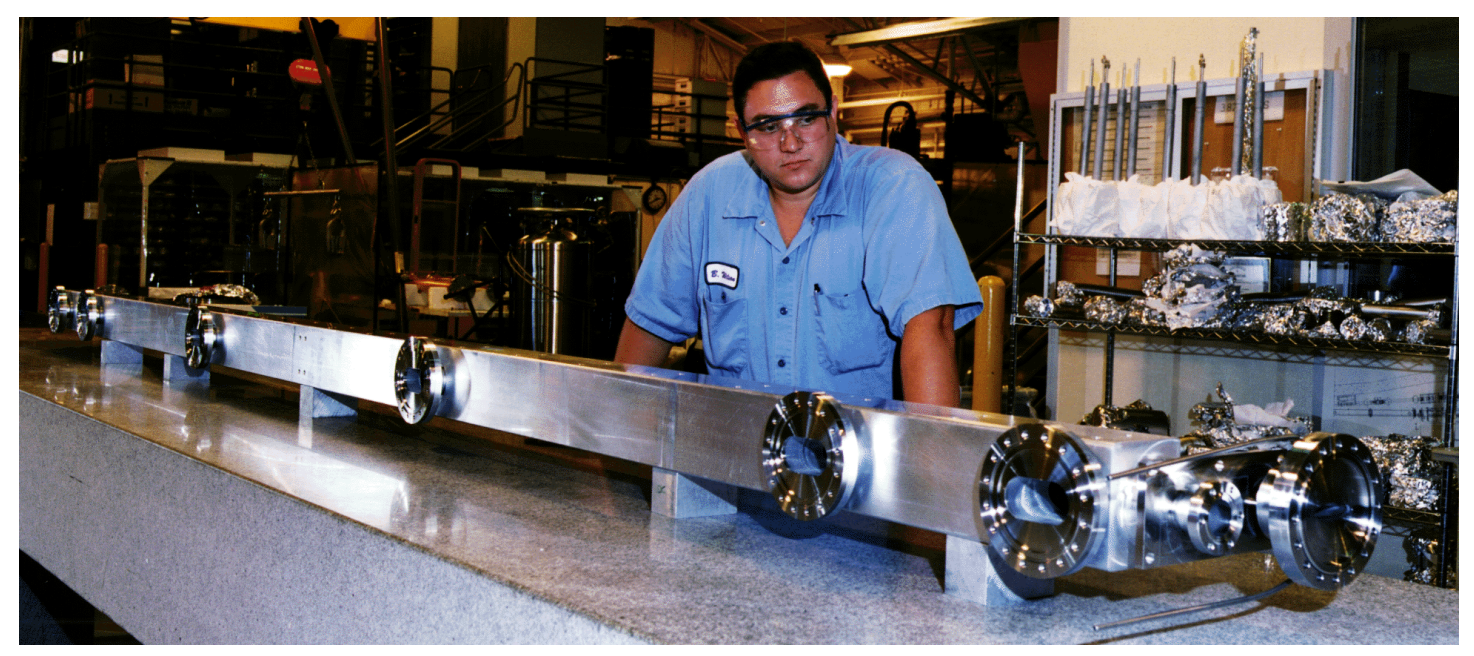

Fig. 5.9 One of the vacuum chambers designed and built for BESSY II. 


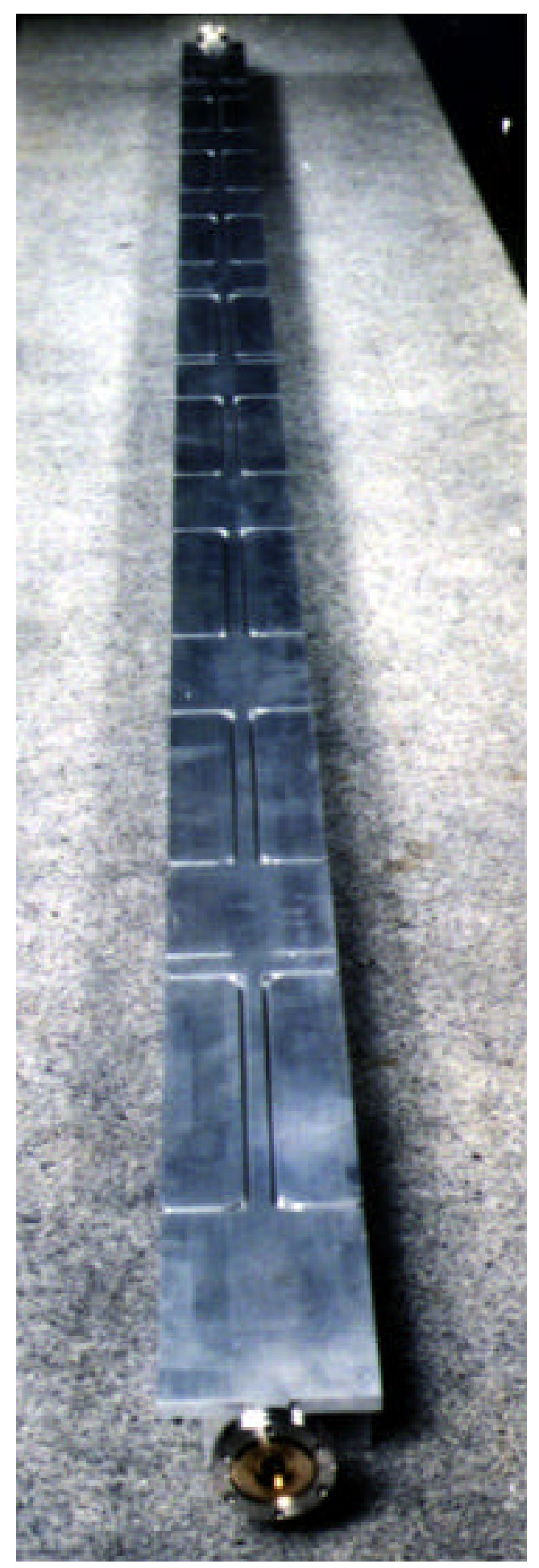

Fig. 5.10 One of the vacuum chambers designed and built for the DESY FEL project. 


\subsection{Beamline Engineering}

\subsubsection{Introduction}

The beamline engineering effort during the facility construction years focused on the design, construction, and commissioning of various beamline components, which included the beamline front ends, the experiment enclosures; various experimentspecific components, etc. These efforts have paid off as measured by the successful operation of user beamlines. During the past year, the focus of the staff working on the R\&D in support of beamline engineering has moved to evaluation of the performance of beamline components, leading to new 
concepts and developments for fututre beamlines. Attention was also given to the new requirements, present and future, to meet goals of the user scientific programs.

One of the major shortcomings in the manufacture of various beamline components by the vendors was their limited success in brazing Glidcop ${ }^{\circledR}$ to various metals. The staff working on beamline engineering hence developed various brazing technologies and capabilities to bridge the gap so that users could successfully begin their scientific programs. In the next section, the brazing furnaces and their capabilities are described.

In order to meet new requirements of the users, conceptual and design changes in the front ends are needed in the newer beamlines planned for construction. These designs and their new capabilities are described in section 5.2.3.

Finally in section 5.2.4, a description of both a laser Doppler displacement meter and an angular encoder with sub-nanoradian sensitivity required for high-resolution monochromators is provided. These unique designs will have a much wider application in the future as users plan more sophisticated experiments.

\subsubsection{Brazing Capabilities for Beamline Components}

\section{Small Brazing System}

A new metallurgical brazing system has been developed by the Beamline Engineering Group that is used primarily for brazing small samples and heat transfer experimental tubes. Due to the small size of the system, it is ideal for brazing small components at a fraction of the cost compared to one of our larger brazing systems. The capability supports special needs of the users, as well as unique $R \& D$ needs for the beamline engineering effort. The small system size allows for rapid heating and cooling, and consequently samples can be processed in a minimal 
amount of time. A Carbolite three-zone clam-shell-type resistance heating furnace is used as the heat source. The three zones are independently controllable via three temperature controllers remotely located on the system control module. A 2-inch (inside diameter) by 4-foot-long clear quartz tube equipped with quartz feedthrough end caps is used for the brazing chamber.

\section{Vacuum Furnace for Large Component Brazing}

Another brazing system has been developed to provide state-of-the-art brazing capabilities for future front-end and beamline components. This new system incorporates a significant number of improvements over our older system to provide several levels of automated control over the brazing process. As before, a thinwalled high-temperature material tube was used for the brazing chamber to minimize heating and cooling times, an essential requirement for brazing Glidcop ${ }^{\circledR}$. A Hastolloy-X rolled and welded tube with a 7 1/2 inch inside diameter and 15 feet long was selected due to the superior hightemperature properties of this material. The long tube length provides the flexibility to braze very long parts.

A $15-\mathrm{kW}$ three-zone Lindburg® clam-shelltype resistance heating furnace is used as the heat source and is mounted with precision slides on a rail system to allow easy movement of the furnace along the tube. The furnace is coupled via a magnet lock to a precision ball screw drive system, which is controllable from a remote keypad and joystick. The controller can be easily programmed from a PC to provide any combination of motion and positioning 
referenced from a "home" position with a maximum speed of 2 inches per second. The motion control system provides the capability to oscillate the oven over long parts to achieve repeatable uniform heating and also provides an absolute position reference for repeating previous braze runs. The magnet lock can be instantly deenergized via a large emergency stop button to decouple the oven from the drive system.

As with the older brazing system, the three oven zones can be individually controlled from remote temperature controllers, however, an "auto-tune" feature has been added to eliminate the need to calibrate the braze run. Another key feature of the new system is a fully automated pump and purge controller. Prior to brazing, the chamber needs to be pumped and purged in a repetitive fashion with a high purity inert gas in order to "wash" contamination from the chamber walls and the component. If done manually, this process is time consuming. The pump and purge system will automatically perform this process, and the number of cycles and pumping times can be changed via keypads on the controller. The gas delivery system accommodates four separate gas sources, typically $\mathrm{Ar}, \mathrm{He}$, and two $\mathrm{N}_{2}$ supplies. The microbleed circuit is used during the brazing process to achieve a reduced atmosphere in the chamber, and the quenching circuit is used to rapidly cool brazed parts. All of these circuits have precision flow-control valves and flow meters for repeatable gas delivery rates. The vacuum aspects of the new brazing system are far superior to the system used previously in this effort.

During the year, several braze techniques for joining stainless steel to GlidCop® were developed. These techniques were used to fabricate several fixed masks that are now installed in the front ends. In addition, some of the components for the SPring- 8 front ends were designed and fabricated, which involved various specialized brazing jobs.

\section{5-ID Ratchet-Wall Collimator Tube Examinations}

In section 2.6.6, a detailed description of the sector 5 front-end problem was provided. This single incidence in which the ratchetwall collimator was distorted required independent examination. The collimator, when isolated for study, showed considerable residual distortion.

The collimator itself was displaced by as much as $9 \mathrm{~mm}$ in the shield wall, with about a 6-mm bend in the tube. When it was examined in the laboratory, the residual bend was only $2.1 \mathrm{~mm}$. In order to understand the source of this distortion, a detailed examination was performed. Corrosion was found in the lead shot surrounding the collimator. Chemical test results showed that most of the corrosion was aluminum oxide deposited on the lead shot. Lead oxides and concrete were also found, although the amounts were small in comparison.

A bend test was designed to simulate possible conditions in the shield wall. As shown in Fig. 5.11, approximately 36,000 N displaced the tube $7 \mathrm{~mm}$ and left a residual bend of $3.2 \mathrm{~mm}$. In the actual front ends, the cause of such force is unclear; however, the pressure would not have been generated if both ends of the collimator were not clamped. As described in section 2.6.6, all ratchet-wall collimators were modified as a preventative measure to allow for changes in the collimator dimensions. The chemical 
(a)

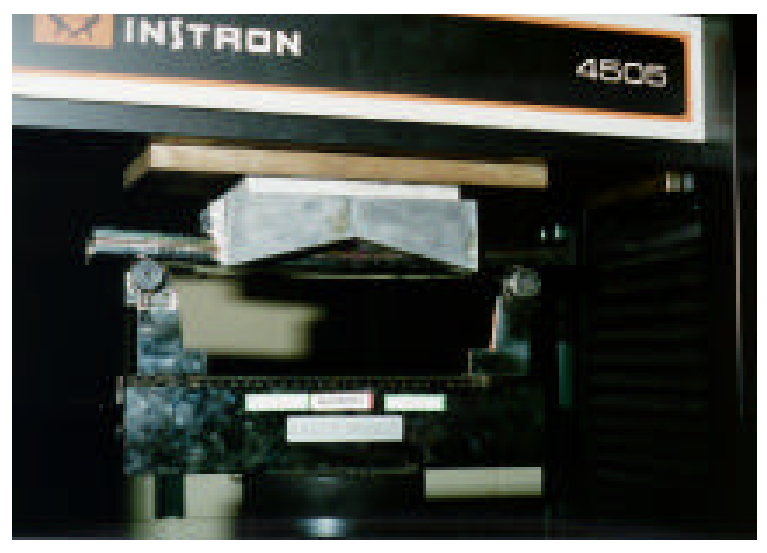

(b)

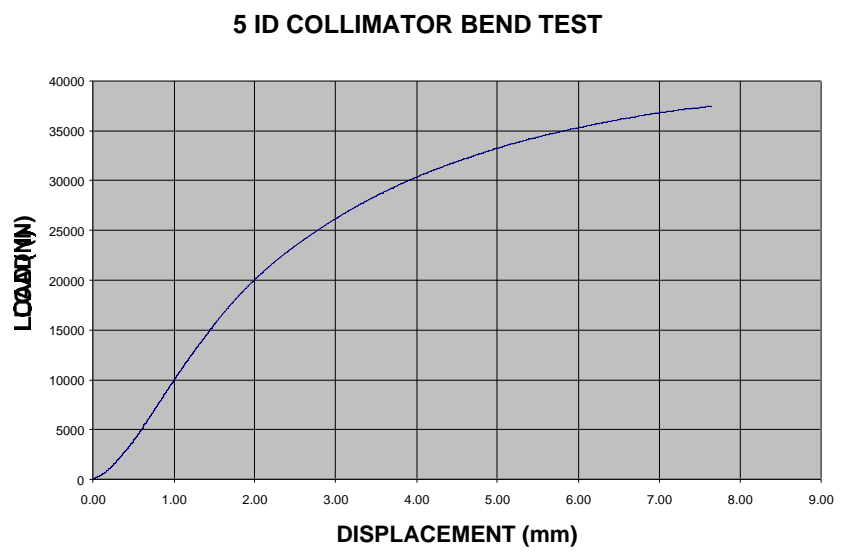

Fig. 5.11 Simulation of ratchet wall collimator distortion. (a) Bending test on the instron; (b) force deflection data.

activity and resulting corrosion inside the ratchet wall is a long-term concern. There are plans to evaluate this systematically during the next few years.

\subsubsection{Beamline and Front-End Design for New Sectors and User Support}

\section{Front-End and Beamline Design for the SRI-CAT 1-ID Backscattering Beamline}

The absence of beamlines in sectors 36 through 40 and the geometry of the components in the APS storage ring tunnel provide a unique opportunity to build a backscattering beamline on the 1-ID source. The concept for this unique capability was developed by David Moncton. The modifications required in the storage ring components to extract the x-ray beam have been completed by ASD. The front end and beamline have been designed for this 1-ID backscattering beamline. A redesign of the 1-ID front end has been completed so that there is minimal impact on SRI-CAT 1-ID beamline operation. Figure 5.12 shows plan and side views of the backscattering beamline layout. In scattering back from a yet-to-be-designed special monochromator in the 1-ID-B station, the beam penetrates the ring wall tangentially through a long vacuum transport. This specially designed 10-m-long vacuum pipe was procured and installed in the wall recently. The long pipe required a special support structure inside the shielding wall to optimize the alignment and positioning capability. These support pieces are manipulated for alignment from the end openings in the ring wall. Fig. 5.13 shows a photo of this support structure. In addition, a mono-beam shutter design, P8-50, has been completed for the 1-ID backscattering beamline, and backscattering monochromator design work is in progress to complete the beamline. 


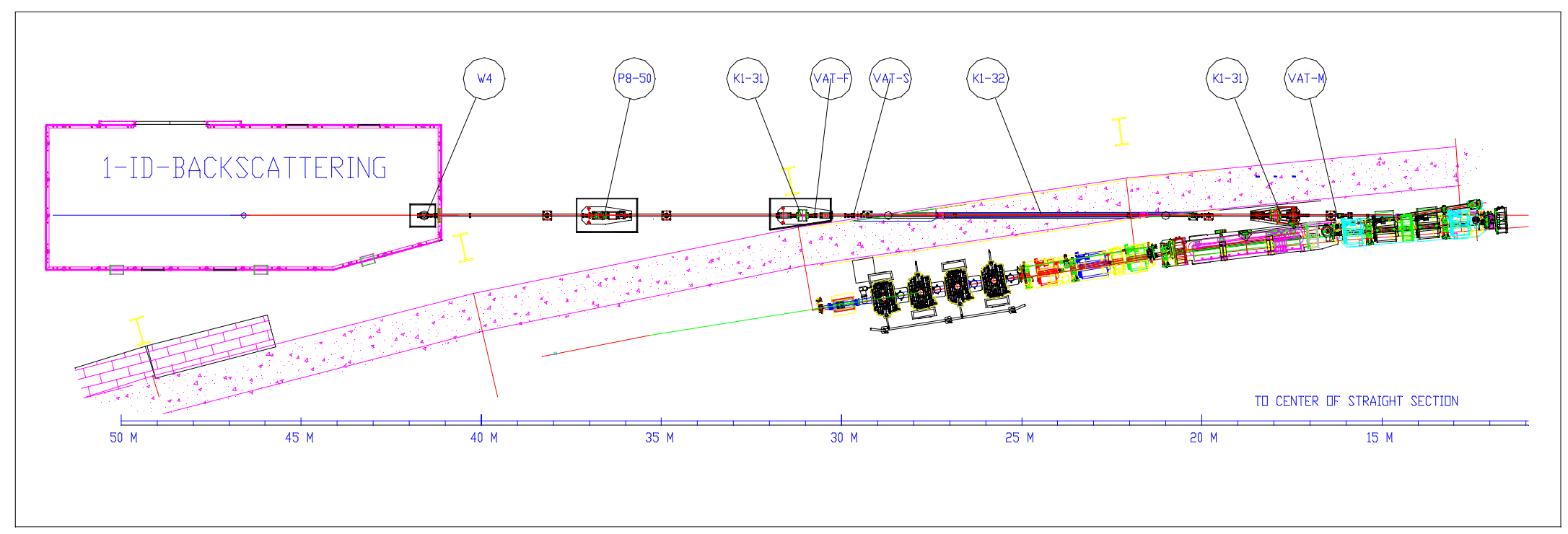

Fig. 5.12 Schematic layout of the 1-ID backscattering beamline. Beam enters from the right and travels to the left. W4: beryllium window, P8-50: monochromatic shutters, K1-31: collimator/differential pumps, VAT-F: fast valve, VAT-S: UHV valve, K1-32: 9-m wall collimator, VAT-M: all metal valve. 


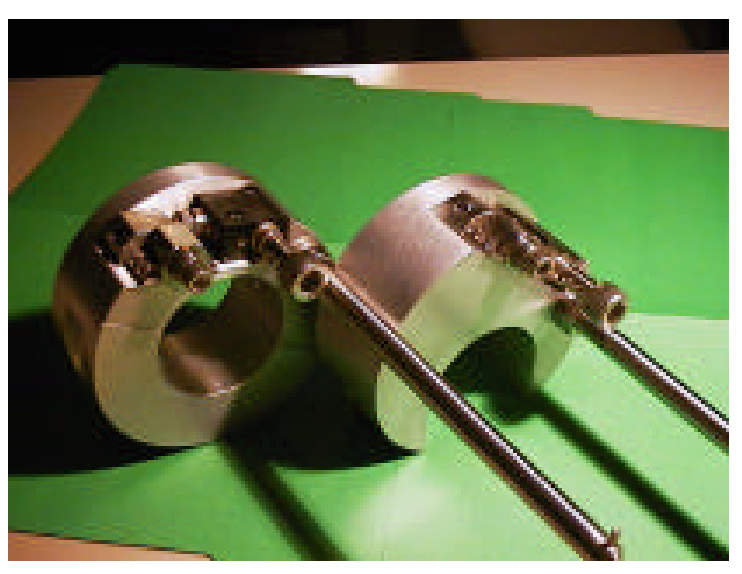

Fig. 5.13 Photo of the special support structure designed for the 10-m-long vacuum pipe in the 1-ID backscattering beamline.

\section{SRI-CAT Sector 4 Beamline Design}

A new sector was granted to SRI-CAT to develop techniques that use $\mathrm{X}$-ray polarization for magnetic studies. The beamlines in this sector utilize unique undulators (see section 5.1.3) with radiation beams separated in the horizontal plane. The components for this set of beamlines on one straight section required special consideration. The design is now complete.

The front end for this beamline is shown in Fig. 5.14. All the 4-ID beamline front-end masks are designed for undulator A operated in the closed gap configuration at 130-mA stored current. Fixed mask M1-40 is made of a Glidcop® cylinder with stainless steel plates on both ends attached by explosive bonding. The first fixed mask of the 4-ID front end, M1-40, is 15.5 meters from the undulator A source. At 130-mA beam current and 11-mm gap, M1-40 will intercept an x-ray beam of $7.03-\mathrm{kW}$ total power with $16.7-\mathrm{W} / \mathrm{mm}^{2}$ peak power density $\left(1.1^{\circ}\right.$ incident angle). Thermal calculations using the ANSYS analytical package for the same operating conditions were carried out for the M1-40, M2-40, and M2-50 fixed masks, and the results are shown in Table 5.3.

A novel feature of this front end is the integrated design of the fixed mask and $\mathrm{x}$-ray beam position monitor (XBPM) as a single unit. A chemical vapor deposition (CVD) diamond-based transmitting XBPM (Fig. 5.15) is mounted on the downstream side of the fixed mask. This new design not only reduces the front-end construction cost significantly but also improves the XBPM performance in rejecting the VUV and soft $\mathrm{X}$-ray beam contamination. A prototype of this new design was tested at the APS 6-ID beamline FOE. Figure 5.16 shows the XBPM readout as a function of the beam vertical position.

Figure 5.17 shows the general layout of the sector 4 beamline branches. A total of six different fixed masks are being designed to allow the two undulators in the sector 4 straight section to be operated independently for two beamline branches. The experiment station design for SRI-CAT sector 4 is complete.

Table 5.3 Thermal calculations ${ }^{a}$ for the M1-40, M2-40, and M2-50 fixed masks.

\begin{tabular}{|l|l|l|l|}
\hline & M1-40 & M2-40 & M2-50 \\
\hline Distance from the Source $(\mathrm{m})$ & 15.5 & 16.2 & 20.0 \\
\hline Incident Angle (degrees) & 1.1 & 1.1 & 1.4 \\
\hline Max. Power Density $\left(\mathrm{W} / \mathrm{mm}^{2}\right)$ & 16.7 & 15.2 & 12.8 \\
\hline Max. Temp. on Glidcop ${ }^{\circ}\left({ }^{\circ} \mathrm{C}\right)$ & 255 & 267 & 260 \\
\hline $\begin{array}{l}\text { Max. Temp. at Cooling Wall } \\
\left({ }^{\circ} \mathrm{C}\right)\end{array}$ & 98 & 100 & 102 \\
\hline
\end{tabular}

a beam current $=130 \mathrm{~mA}$, undulator gap $=11 \mathrm{~mm}$ 


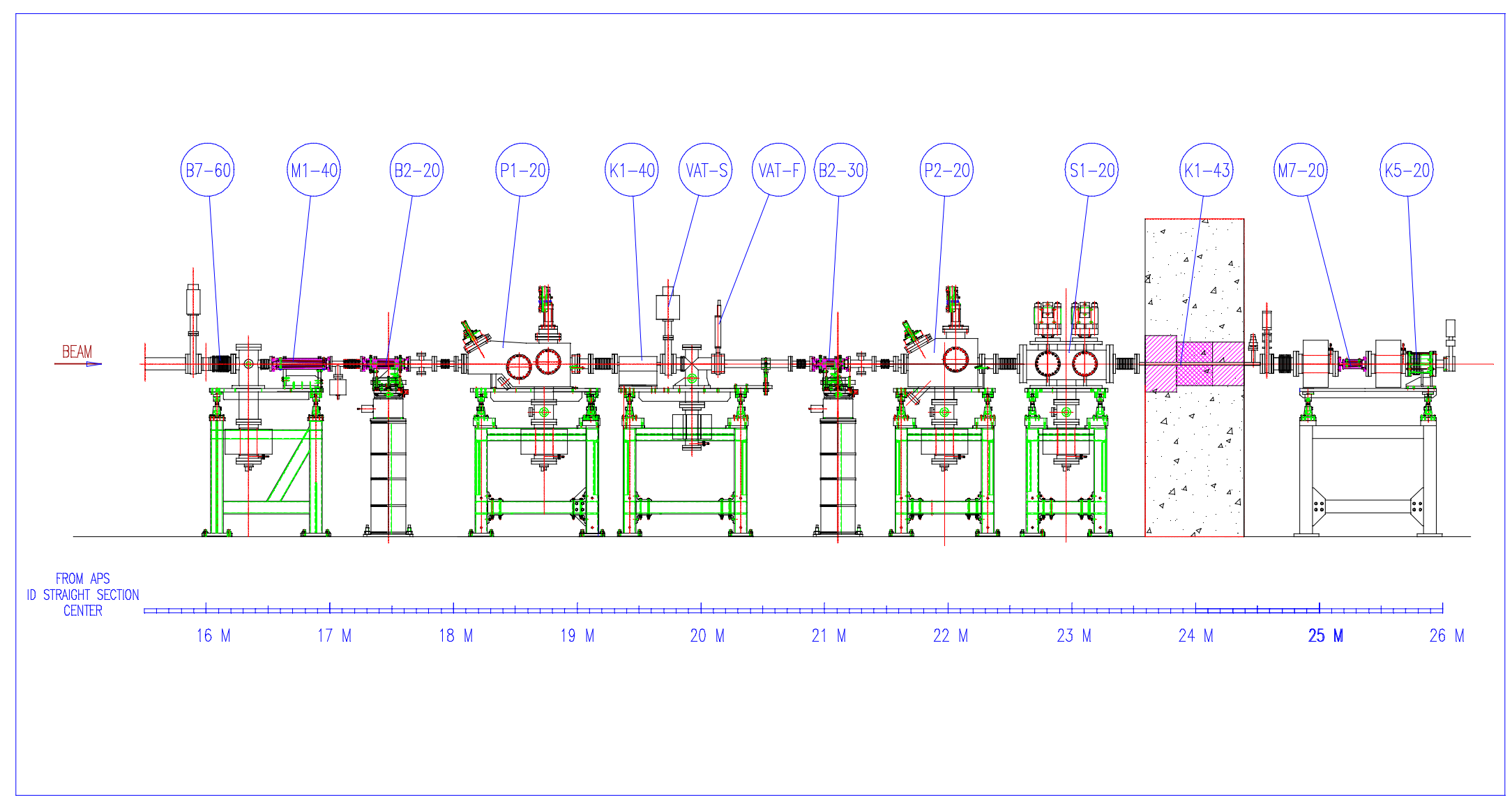

Fig. 5.14 Schematic layout of the 4-ID beamline front end. Beam enters from the left and travels to the right. B7-60: BM mask/bellows, M1-40: ID fixed mask, B2-20: first XBPM/fixed mask, P1-20: first photon shutter, K1-40: collimator, VAT-S: UHV valve, VAT-F: fast valve, B2-30: second XBPM/fixed mask, P2-20: second photon shutter, S1-20: safety shutter, K1-43: wall collimator, M7-20: fixed mask/differential pumps, K5-20: invacuum collimator. 


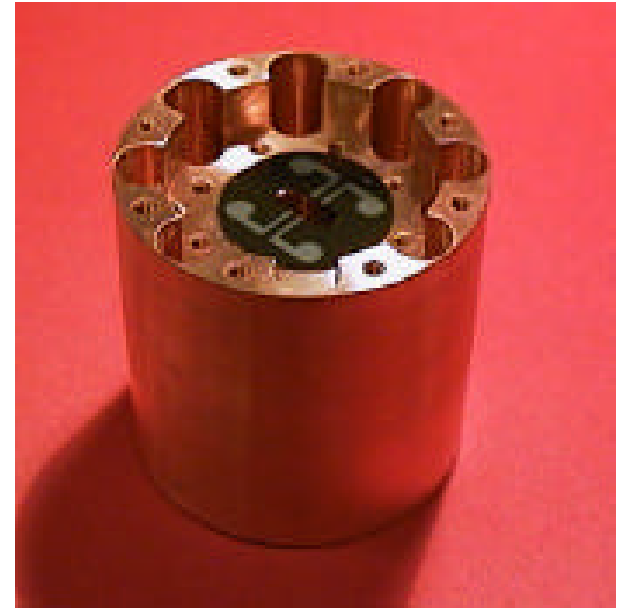

Fig. 5.15 CVD-diamond-based transmitting XBPM, which is mounted on the downstream side of the fixed mask when installed on a beamline.

\section{Beamline Components for CATs}

Many beamline mechanical components have been designed and fabricated for many CATs. The ability to easily modify standard designs to meet unique goals and fabrication capabilities has generated such requests. The components in demand are slits (L5-90) and monchromatic beam shutters (P8-20). The CATs benefiting from this capability include COM-CAT, CHEMAT CARS, MU-CAT, and UNI-CAT, in addition to SRI-CAT.

\section{Design Exchange}

The XFD Design Exchange (DX) went through significant upgrading and

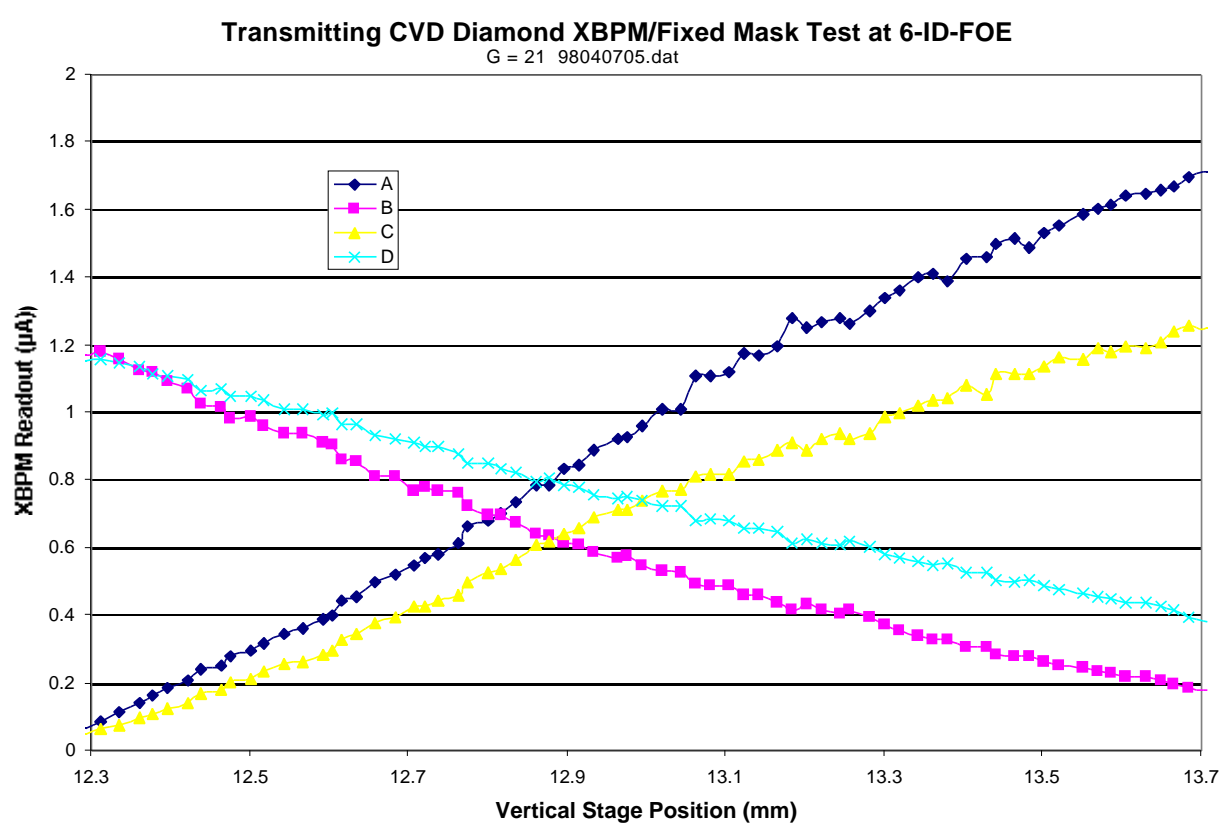

Fig. 5.16 The XBPM readout as a function of the beam vertical position. 


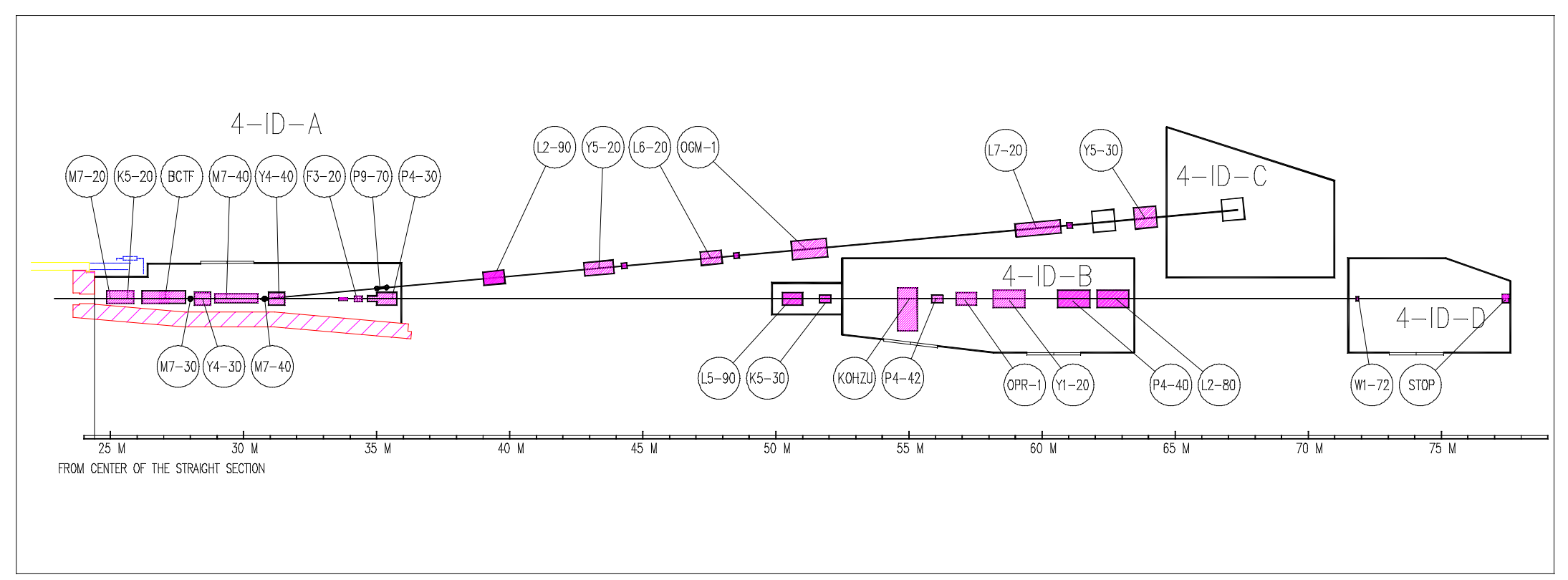

Fig. 5.17 Schematic layout of the sector 4 beamline capable of delivering two undulator beams simultaneously. Beam enters from the left and travels to the right. M7-20: fixed mask/differential pumps, K5-20: in-vacuum collimator, BCTF: beamline component test facility, M7-30: fixed mask plug, Y4-30: first horizontal mirror for station 4-ID-C, M7-40: dual-beam fixed mask, M7-50: fixed mask plug, Y4-40: second horizontal mirror for 4-ID-C, F3-20: filters, P9-70: pink-beam shutters, P4-30: white-beam integral shutters, L2-90: pink-beam slits/XBPM, Y5-20: first vertical mirror for 4-ID-C, L6-20: entrance slit, OGM-1: grating monochromator, L7-20: exit slit, Y5-30: refocusing mirror for 4-ID-C, L5-90: white-beam slits, K5-30: in-vacuum collimator, KOHZU: crystal monochromator, P4-42: white-beam stop, OPR-1: $x$-ray phase retarder, Y1-20: x-ray mirror, P4-40: white-beam integral shutters, L2-80: pink-beam slits, W1-72: beryllium window, STOP: beam stop. 
renovation during the year. The following improvements were made in hardware and software.

We upgraded to Solaris 2.6 (Y2K compliant) and upgraded to a 22-GB hard drive for drawing storage; we are officially on R13 of AutoCad, supporting SAMBA services for file and print sharing. New Legato Networker Server software is now used to back up PCs, and work is still in progress on conversion from "A" numbers to "long filenames" for drawing libraries.

All design engineers and designers contributing to the DX are using Mechanical Desktop/AutoCAD 14. The PC stations available to mechanical engineers and design engineers were upgraded to meet the work requirements.

Scheduled backups take place on a regular basis for 60 PCs (out of 70 licenses available), and smooth and reliable storage, retrieval, and recovery of files are possible on server and workstations.

\subsubsection{Laser Doppler Angular Encoder with Sub-Nanoradian Sensitivity}

Recently, the demands for motion and control of optical elements increased. For example, in $\mathrm{x}$-ray scattering experiments involving ultrahigh resolution (sub-meV at 10-30 keV), the motion control on the monochromating crystals has to be at the 1-to-10 nanoradian level or better (Toellner et al., 1997). However, if closed-loop feedback devices are used, the required resolution for the motion sensor (angular encoder) will be in sub-nanoradian level over a measuring range of 8 degrees.
There is, at present, no commercially available angular encoder with subnanoradian resolution over an 8-degree measuring range. In the field of gratingbased encoders, one of the best available products is ROD-800 from Heidenhain, which has 175-nanoradian resolution with a 360-degree measuring range when coupled with an AWE 1024 interpolator (Heidenhain, 1996). As for commercial laser interferometers, the Hewlett Packard HP-5527B (Hewlett Packard, 1996) and Zygo ZMI-1000 (Zygo, 1996), provide a 20-100 nanoradian angular resolution from a few degrees up to 20 degrees angular measuring range. Although some tiltsensors, such as the Applied Geomechanics Model-520, have 10-nanoradian resolution, they cover a measuring range of less than 0.01 degree with a very long measurement setting time (0.1-30 seconds).

In a laboratory setup based on a polarization-encoded Michelson interferometer system, a few nanoradian resolution has been achieved with a large setup (size about $610 \mathrm{~mm} \times 1220 \mathrm{~mm}$ ). A typical sine-bar configuration was used in this design to convert the angular measurement to a linear displacement measurement. The dimension of the sine bar was restricted to less than $310 \mathrm{~mm}$ in length by the monochromator structure and system stability limits. To achieve sub-nanoradian angular resolution, the resolution needed for the linear displacement measurement has to be in the near-Angstrom range. The overall dimensions of the encoder system are critical to the performance of the closedloop feedback system. In general, however, the large setup size will cause complications for the system's thermal and mechanical stability. 
The laser Doppler displacement meter (LDDM) is based on the principles of radar, the Doppler effect and optical heterodyning (Wang et al., 1987). We have chosen an LDDM as our basic system not only because of its high resolution (10 $\mathrm{nm}$ typical) and high measuring speed but also because of its unique performance-independent of polarization-which provides the convenience to create a novel multiplereflection-based optical design to attain near-Angstrom linear resolution extension.

Figure 5.18 shows the self-aligning 3-D multiple-reflection optical design for the LDDM system resolution extension. In this design, the heterodyning detector is housed coaxially inside the frequency-stabilized laser source. Instead of a typical single reflection on the moving target, the laser beam is reflecting twenty-four times between the fixed base and the moving target. The laser beam, which is reflected back to the heterodyning detector, is frequency-shifted by the movement of the moving target relative to the fixed base. With same LDDM laser source and detector electronics, this optical path provides twelve-times resolution extension power for the linear displacement measurement and

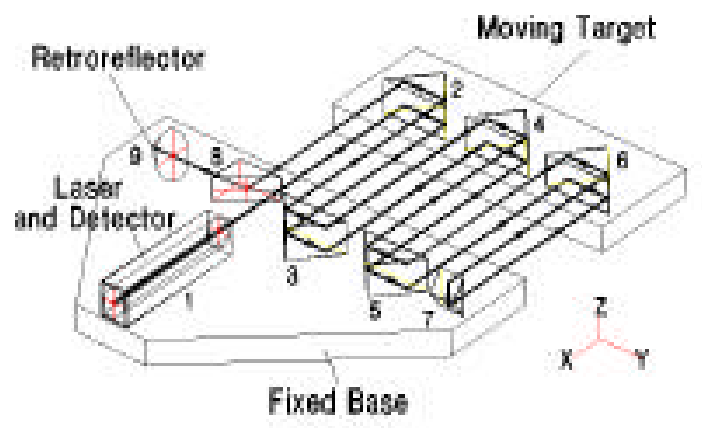

Fig. 5.18 The self-aligning 3-D multiplereflection optical design for the LDDM system resolution extension. encoding. The 3-D optical path configuration results in a compact and integrated optical design that optimizes the system's antivibration performance, which is critical for sub-nanoradian resolution in measurements.

Supported by a Laboratory Directed Research and Development (LDRD) award, a prototype laser Doppler angular encoder (LDAE) has been developed for highenergy-resolution $\quad x$-ray scattering applications at the APS undulator beamline 3-ID. We have modified the monochromator (AAG-100, manufactured by Kohzu Seiki Co., Japan) sine bar and related structure for the LDAE assembly. Figure 5.19 shows the configuration of an actual LDAE system with twenty-four multiple-reflections on the one end of the sine bar, which rotates the shaft on which the asymmetrically cut

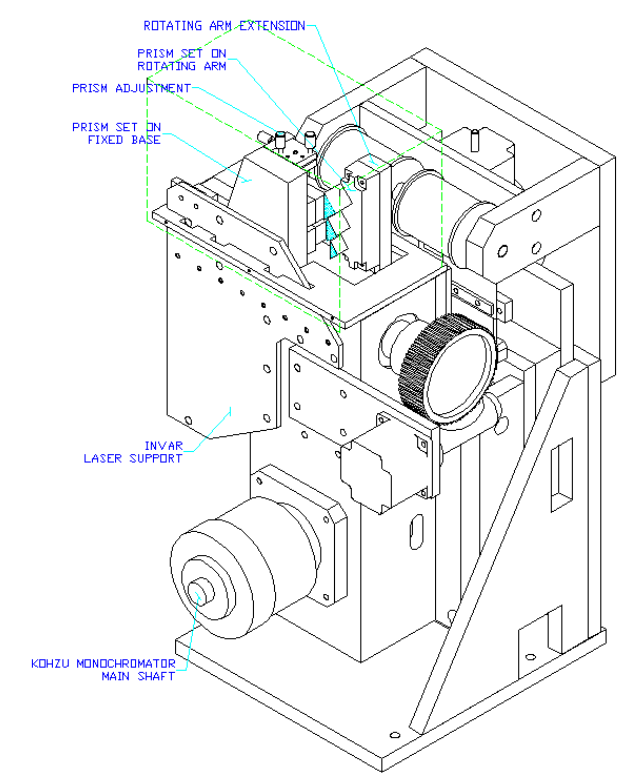

Fig. 5.19 Configuration of an actual LDAE system with twenty-four multiple-reflections on the one end of the sine bar, which rotates the shaft on which the asymmetrically cut crystals are mounted. 
crystals are mounted. To control the system's thermal stability, a water-cooling jacket was attached to the laser source housing. Figure 5.20 shows a photograph of the monochromator with the LDAE.

Figure 5.21 is the plot of the test results that correlates the performance of our LDAE with a Heidenhain ROD-800 optical encoder with a 2-arc sec accuracy and 175-nanoradian resolution. The slope of the correlation data in Fig. 5.21 shows that our LDAE has a 0.2762 nanoradian per count readout sensitivity. A $100 \mathrm{mrad} / \mathrm{sec}$ rotation speed was tested for a laboratory setup in the 8-degree measuring range without any encoder miscounting.

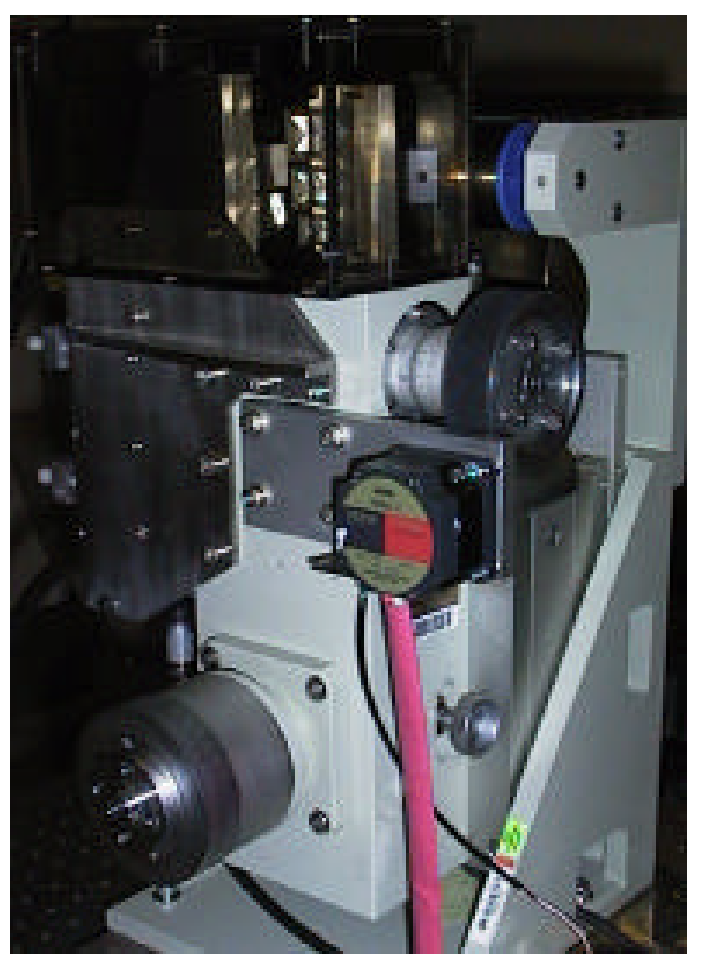

Fig. 5.20 Photograph of the monochromator with the LDAE.

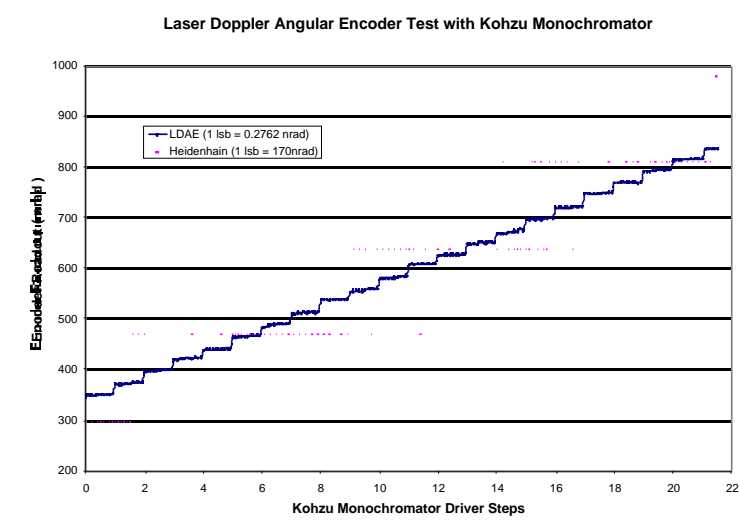

Fig. 5.21 Plot of the test results that correlates the performance of our LDAE with a Heidenhain ROD-800 optical encoder with a 2-arc sec accuracy and 175-nanoradian resolution.

It is very difficult to prove a sub-nanoradian system resolution experimentally in an open-loop system, because of the thermal and mechanical vibration noises. However, with a commercial piezoelectric transducer (PZT) driver, such as a Queensgate NPS3330, we have made an open-loop test with two 6.6-nanoradian motion steps. During this test, the same sine bar and the LDAE moving target were driven by a Queensgate PZT drive. Figure 5.22 is the plot of the test results that correlates the readout sensitivity with the Queensgate PZT driver with two 6.6-nanoradian jumps. The error bar on Figure 5.22 reflects the PZT driver system noise, which was about 1.9 nanoradians peak-to-peak. Recently, work has been initiated on a driving system with closed-loop configuration and 1-nanoradian resolution. 
LDAE Test with Queensgate PZT Driver Two 6.6 nrad Jumps

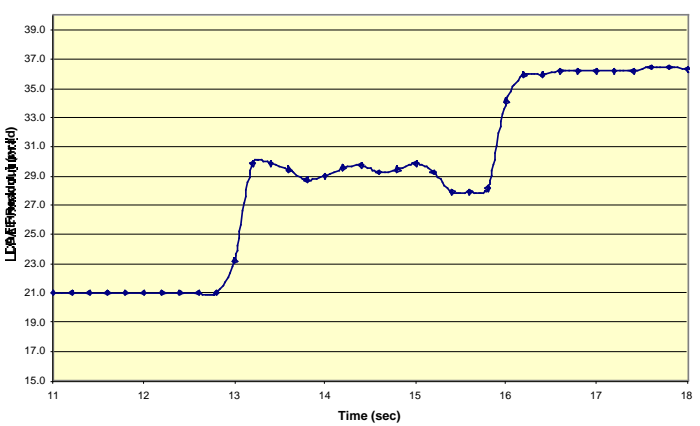

Fig. 5.22 Plot of the test results that correlates the readout sensitivity with the Queensgate PZT driver with two 6.6-nanoradian jumps. 


\subsection{X-ray Optics Development}

\subsubsection{Introduction}

During the past years, the focus of the staff working on $\mathrm{x}$-ray optics was almost exclusively on the development of highheat-load monochromators and mirrors and their associated hardware. That work has paid off in the successful development of cryogenically cooled optical systems (crystals and liquid nitrogen pumps), watercooled diamond monochromators, and contact-cooled mirrors with post-mirror, water-cooled monochromators. Many of the other CATs at the APS currently use cryogenically cooled silicon monochromators and a liquid nitrogen $\left(\mathrm{LN}_{2}\right)$ pumping system, specified by XFD staff and first tested at the APS on the 1-ID beamline of SRI-CAT. To date, the cryogenic monochromators and pumps have performed very well with minimal lost beam time due to inadequate operation of the cryosystem. A few of the CATs have opted for a mirrorfirst geometry utilizing side-cooled silicon mirrors, also developed by the XFD staff, as the first optical components in their beamlines. These too have performed as designed.

We are continuing to improve the performance of existing optical components, some of those programs are described in detail below, while simultaneously looking ahead to components capable of operation under even higher powers and power densities from enhanced storage ring performance, such as higher current and smaller vacuum chamber gaps, and/or 5-meter insertion devices. Although this study into higher power optical components is not yet complete, some of the initial results of that study have been included in this report.

Albeit the design and testing of high-heatload optical components continue to occupy a good part of the time, some staff time has been allocated to x-ray optics developments not directly related to high-heat-load optical systems. Sagittally focusing crystals, x-ray interferometers, compound refractive lens, and use of gradient d-spacing crystals are but a few of the projects that are currently under development in XFD.

It should be pointed out that an important side benefit or spin-off of the high-heat-load monochromator development program was the establishment at the start of the program of a high-quality facility for the fabrication and characterization of single-crystal optical components. This facility continues to fabricate single-crystal optical components for high-heat-load optics development by XFD staff in addition to providing its services to all CATs at the APS. (See chapter 4 for more details on the capabilities and accomplishments of the x-ray optics fabrication and metrology laboratory.) 


\subsubsection{Cryogenically Cooled Silicon Monochromators}

Cryogenically cooled silicon monochromators have been successfully in use at the 1-ID beamline for several years now. In the past year, our efforts have been directed towards improving the overall performance of the cooled monochromator system. Specifically, ongoing studies include investigation of the influence of geometrical parameters (i.e., crystal shape) on the performance of the component, cooling of the second crystal in the doublecrystal monochromator (DCM) to reduce beam walk as a function of energy, and the use of liquid nitrogen reliquifiers to minimize the need to replenish the $\mathrm{LN}_{2}$ supply. These studies are discussed in detail below.

Over the past year, we have initiated a program to explore the dependence of and to optimize the geometrical parameters on the thermal performance of the monochromator using finite element analyses (FEA). Our original design for a cryogenically cooled silicon monochromator called for a thin web to be fabricated on a robust block of silicon so that much of the incident beam power would be transmitted rather than being absorbed in the monochromator. An earlier experiment conducted by the high-heat-load team (Rogers et al., 1996), showed no difference in $\mathrm{x}$-ray performance when the incident white beam impinged on the thin web or on a thick part of the silicon block (see Fig. 5.23). To better understand this, a model was developed to study the response of a simple block of cryogenically cooled silicon subjected to the same conditions as a thin one. The FEA results showed indeed that, under the current operational conditions, a simple cooled silicon block

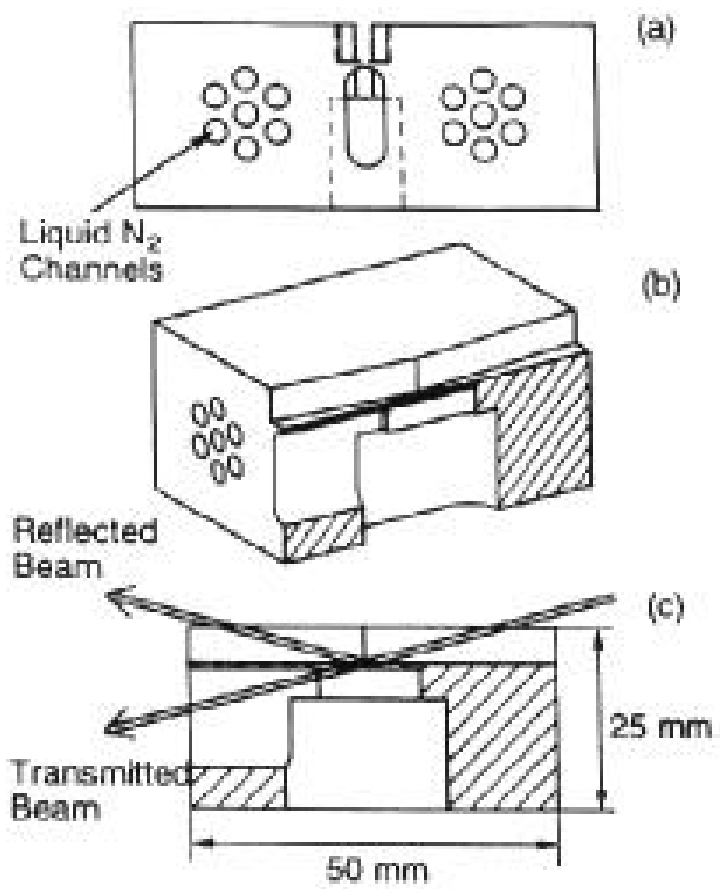

Fig. 5.23 The original design for a cryogenically cooled silicon monochromator at the APS. A thin web was fabricated by milling a channel from the top of the block and removing material below the diffracting surface. This would allow a portion of the beam to pass through the monchromator and reduce the total heat absorbed. Experimental results showed that, at $100 \mathrm{~mA}$ and an undulator gap of $11 \mathrm{~mm}$, the monochromator functioned perfectly even when the beam was allowed to strike the thick potion of the crystal (out of the channel on the top surface) resulting in a much higher absorbed power load.

should perform as well, if not better, than one with a thin web fabricated into it. The magnitude of the mapping distortions depends on the cooling geometry and efficiency. In the case of a thin crystal, although less total heat is absorbed, heat flow is restricted by the dimension of the thin web, while in the thick crystal more 
volume is available for the heat to spread, compensating for the additional absorbed power. In the case of a crystal made of a simple silicon block, the bowing component of distortion can be minimized by making the crystal as thick as possible; this may not be the case for the thin web design. Using a simple block leads to considerable saving in cost and fabrication time.

Other geomtrical parameters being investigated include overall crystal size (2-D simulations showed the crystal size has to be as large as possible in order to spread the heat and lower the crystal temperature), the size of the thin portion of the crystal relative to the beam footprint (the thin web should be only slightly larger than the beam footprint), and the location of the beam on the thin web (the beam footprint should be as close possible to the crystal thick back wall).

To maintain the monochromatic beam parallel to the incident beam as a function of monochromatic beam energy (and hence have a true fixed offset monochromator), cryogenic cooling of the second crystal necessary. Otherwise the d-spacing mismatch, due to the first crystal being ait cryogenic temperature and the secon crystal at room temperature, would cause the monochromatic beam to move (vertically) a function of energy. For Si (111) crystas going from $6 \mathrm{keV}$ to $18 \mathrm{keV}$, the motion about $4 \mathrm{~mm}$ when the experiment station $30 \mathrm{~m}$ from the monochromator. We have successfully implemented a cryogenic cooling scheme for the second crystal. This scheme involves directly cooling the second crystal mounting block (Invar) in a parallel plumbing arrangement with the first crystal. The second crystal is mounted on the cooling block via clamps and thermal contact is achieved via a layer of indium/gallium. Although the indium/ gallium layer freezes at cryogenic temperatures, it does not appear to strain the second crystal. Temperature measurements show that, during the initial crystal cool down of the DCM, both the first and second crystals show the same cooling behavior and both crystals reach cryogenic temperatures of $\sim 90 \mathrm{~K}$ in about 20 minutes. Furthermore, even though the second crystal stage is now connected to the first crystal via the additional cryogenic cooling lines, our measurements do not show any increase in flow-induced vibrations for the DCM system. However, due to the stiffness of the additional cryogenic cooling lines, the DCM is operated in a "fixed Z2" mode, in which the second crystal does not translate; the beam is allowed to walk on the face of the second crystal, which is $160 \mathrm{~mm}$ long. The measurements confirm that the monochromatic beam does indeed stay at a fixed height as the monochromator energy is varied over the range from 6 to $20 \mathrm{keV}$ (see Fig. 5.24).

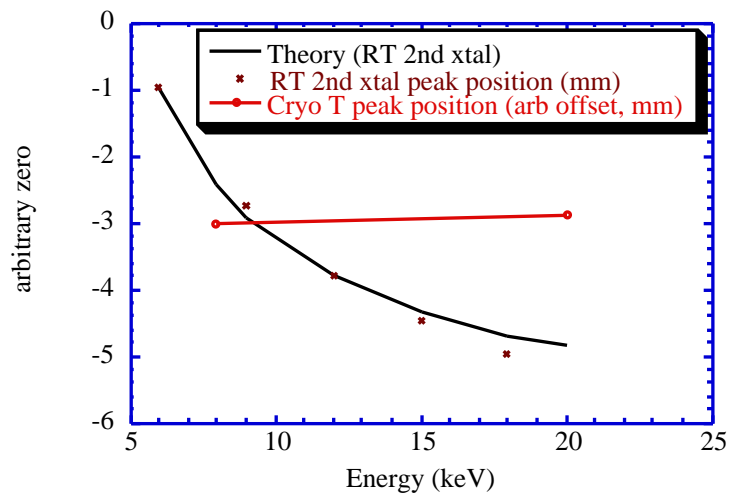

Fig. 5.24 Beam height in station 1-ID-C, as a function of the DCM energy, for a room temperature (RT, i.e., uncooled) second crystal $(x)$ and a cryogenically cooled second crystal (o). 
Although the application of cryogenic cooling has been an overwhelming success, the system does have some overhead associated with its operations. One of these overhead items is replacement of liquid nitrogen that has been lost from the system due to boil-off in the heat exchanger and to system losses. Depending on the crosssectional size of the incident white beam and the setting of the undulator A gap, the cryogenic system requires approximately 180 liters of $\mathrm{LN}_{2}$ per day. This 180 liters includes the system losses (through insufficient insulation, for example, and is estimated to be in the 80-100 $\mathrm{W}$ range) and, depending on the white-beam slit size and undulator gap, that due to the power absorbed by the crystal, which can be in excess of $500 \mathrm{~W}$. (At the highest power, the system requirements can double to 360 liters per day.) Currently there is no distributed $\mathrm{LN}_{2}$ in the experiment hall to provide for the $\mathrm{LN}_{2}$ boil-off. Therefore to minimize the need to constantly change $\mathrm{LN}_{2}$ supply dewars, we have been testing the use of reliquifiers to recapture the $\mathrm{LN}_{2}$ boil-off. The reliquifaction system we have at present consists of two cold heads, each of which is capable of extracting $180 \mathrm{~W}$ from the gaseous nitrogen. As you can see, unless the undulator is running at a very large gap and/or with small slits, the capacity of the cold heads is not sufficient, and we still need to have a $\mathrm{LN}_{2}$ supply tank to supplement the reliquifiers. However, with the addition of the reliquifiers, our need to change/resupply the $\mathrm{LN}_{2}$ tanks has been considerably reduced from about one per day to about one every 3 or 4 days. Efforts to improve the reliability and efficiency of the cryogenic system continues with particular effort on improvements in the efficiency of the cryopumping system.

\subsubsection{Diamond Monochromators}

A water-cooled diamond high-heat-load monochromator was installed as a permanent component in the sector 3 ID beamline in May 1997. The monochromator consists of two synthetic type $1 \mathrm{~b}$ diamond plates, $7 \mathrm{~mm}$ by $5.5 \mathrm{~mm}$ by $0.4 \mathrm{~mm}$ in size, oriented along the (111) direction. Recently a 5-mm (beam aperture) ID vacuum chamber, which allows a minimum undulator magnetic gap of $8.5 \mathrm{~mm}$, was installed in the 3-ID beamline straight section. In this configuration, the calculated maximum power and surface power density from the $2.7-\mathrm{cm}$-period undulator that are absorbed by the first crystal are $20 \%$ and $35 \%$ larger, respectively, than for undulator A with a minimum gap of $11 \mathrm{~mm}$. During the January 1998 run, we tested the thermal performance of the monochromator under this enhanced heat load. Figure 5.25 shows the measured FWHM of the (111) and (333) double-crystal rocking curves as a function of the energy of the (111) reflection, E(111). The undulator gap was changed so that the first harmonic energy corresponded with $\mathrm{E}(111)$. The calculated maximum absorbed power was $46 \mathrm{~W}$ at $10 \mathrm{keV}$ (13.5-mm gap), and the calculated maximum absorbed surface power density was $6.8 \mathrm{~W} / \mathrm{mm}^{2}$ at $9 \mathrm{keV}$ (12.4-mm gap). The absorbed power and surface power density decrease as a function of energy for $\mathrm{E}>10 \mathrm{keV}$. We see no thermal effects on the measured FWHM as a function of the absorbed power or power density. One possible explanation for the increasing deviation from theoretical value of the (111) rocking curve FWHM as a function of energy is near-surface imperfections/defects in the crystals because the beam footprint on the crystal increases 


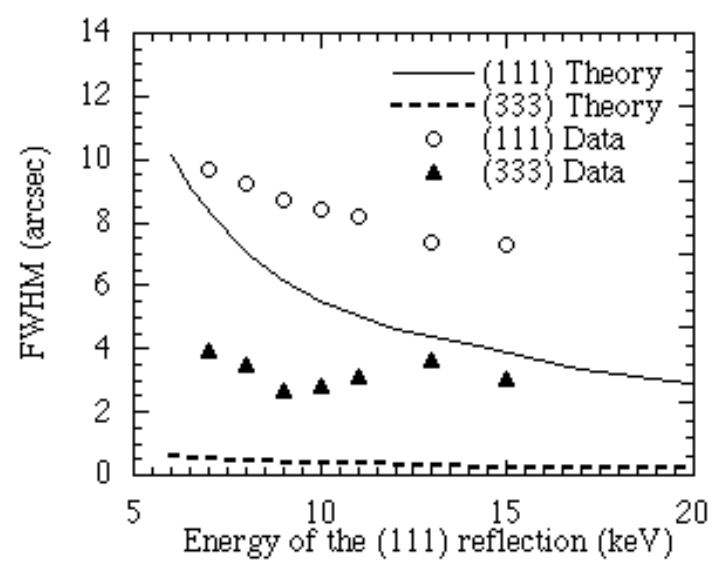

Fig. 5.25 Calculated and measured values for the FWHM of the diamond doublecrystal rocking curves as a function of $E(111)$, the energy of the (111) reflection. Data were taken simultaneously for the (111) and (333) reflections; the energy of the (333) reflection is three times the abscissa value. The undulator gap ranged from $10.6 \mathrm{~mm}$ to $20.2 \mathrm{~mm}$ and was changed so that the first harmonic energy corresponded to E (111). The storage ring current ranged from 93 to $90 \mathrm{~mA}$.

as the energy increases. Such imperfections would be more noticeable in the (111) reflection than in the (333) reflection, since the former has a shorter extinction length.

Future plans for the diamond program include the evaluation of larger synthetic plates $(8 \mathrm{~mm}$ by $5 \mathrm{~mm}$ ) of type IIa that will be delivered by Sumitomo in late 1998, and the development of a cryogenic diamond monochromator for operation under the higher heat loads resulting from enhanced storage ring performance.

\subsubsection{High-Heat-Load Optics for the Future (or Enhanced Storage Ring Operation)}

Efforts have been continuing for many years now in developing analytical and numerical models to predict and optimize performance of high-heat-load monochromators for the APS. The method often used to calculate thermal and mechanical strains is finite element analysis (FEA). This technique in conjunction with simple mathematical models can provide very accurate predictions of the temperature, stress, and strain fields for complicated geometry and boundary conditions, which can then be used in diffraction simulation to calculate the rocking curve of the monochromator. This can save a considerable amount of time and money before building and testing of prototype monochromators. FEA modeling is particularly valuable when no adequate source is available for testing. Currently we are carrying out detailed thermal and structural finite element analyses to predict the performances of water-cooled diamond and liquid-nitrogen-cooled silicon monochromators under power loads that might be available under future operating conditions of the APS. Since it is difficult to predict with accuracy the future operational modes of the APS, we have chosen three scenarios that could be expected in the not-too-distant future (although scenerio (a) in fact already exists on the 3-ID line). These are:

a) an undulator with a $2.7-\mathrm{cm}$ period and a minimum gap of $8.5 \mathrm{~mm}$ and 100-mA beam

b) undulator $\mathrm{A}$ operating with a minimum gap of $8 \mathrm{~mm}$ and $100-\mathrm{mA}$ stored beam 
c) undulator A operating with gap of $10.5 \mathrm{~mm}$ and $200-\mathrm{mA}$ stored beam (This is equivalent to a 5-meter undulator with 100-mA stored beam.)

To date we have modeled only the current configurations of high-heat-load optical elements (cryogenically cooled silicon monochromtators, water-cooled diamond monochromators, and side-cooled silicon mirrors). The monochromator energy range and ID gap combination are chosen so that they correspond to the worst case scenarios in term of absorbed surface power and power densities, and the beamlines are assumed to be windowless. We started out the modeling program looking at diamonds. We carried out simulations for two types of diamonds: type $1 \mathrm{~b}$ and type IIa, using thermal conductivity values of 15 and $20 \mathrm{~W} / \mathrm{cm}-\mathrm{K}$, respectively. The beam size is assumed to be $1.2 \mathrm{~mm}$ (vertical) by $2 \mathrm{~mm}$ (horizontal). The source parameters and power loads used in the FEA are given in Table 5.4.
The calculated (angular) distortions in all cases are smaller than the FWHM of the rocking curve of a perfect DCM. It is therefore expected that the water-cooled diamond monchromator performance will not be altered under the severe heat loads expected from future operating scenarios.

Work is ongoing for the crygencially cooled silicon monochromators (a more difficult task due to the more complicated geometry and nonlinearities associated with the strongly temperature-dependent thermal conductivities and coefficients of thermal expansions). However initial results indicate that the current design may not be adequate for the highest powers and power densities being considered above.

While moderate heat load increases (up to about $30 \%$ ) on the mirrors of present design can be tolerated at a cost of about a $50 \%$ increase in slope error, more significant increases in the heat load can lead to unacceptable temperature, slope errors, and stresses. We have embarked on improving

Table 5.4 Parameters used for the FEA of the diamond monochromator. The absorbed power and power density are calculated for $0.4-\mathrm{mm}$-thick diamond and for $1.2 \mathrm{~mm} \times 2 \mathrm{~mm}$ beam size at $30 \mathrm{~m}$ from the source.

\begin{tabular}{|l|c|c|c|c|c|c|c|}
\hline \multicolumn{1}{|c|}{ Source } & $\begin{array}{c}\text { Gap } \\
(\mathrm{mm})\end{array}$ & $\mathrm{K}$ & $\mathrm{E}(\mathrm{keV})$ & $\begin{array}{c}\text { Aragg } \\
\text { Angle } \\
(\mathrm{deg})\end{array}$ & $\begin{array}{c}\text { Current } \\
(\mathrm{mA})\end{array}$ & $\begin{array}{c}\text { Absorbed } \\
\text { Power } \\
(\mathrm{W})\end{array}$ & $\begin{array}{c}\text { Surface } \\
\text { heat flux } \\
\left(\mathrm{W} / \mathrm{mm}^{2}\right)\end{array}$ \\
\hline $\begin{array}{l}\text { Undulator A, } \\
\text { period=2.7 cm }\end{array}$ & 8.5 & 2.181 & 5.1 & 36.3 & 100 & 57 & 14.4 \\
\hline Undulator A, & 8.0 & 3.57 & 3.5 & 59.3 & 100 & 33 & 11.7 \\
period=3.3 cm & & & 17.0 & 10.2 & & 72 & 5.4 \\
\hline Undulator A, & 10.5 & 2.7 & 3.5 & 59.3 & 200 & 73 & 27.1 \\
period=3.3 cm & & & 17.0 & 10.2 & & 149 & 11.2 \\
\hline
\end{tabular}


the design of a contact-cooled mirror aimed at handling, with acceptable performance, up to three times the current heat load. The key features of this mirror design are (1) introduction of a pair of notches in the mirror substrate (see Fig. 4.8 in chapter 4) for a more effective establishment of thermal moment balance in the substrate, (2) replacement of the indium foil used as interstitial material (between the copper cooling and silicon mirror) with $\mathrm{In} / \mathrm{Ga}$ eutectic for a more efficient heat transfer, and (3) increasing the cooling block width for reducing substrate temperature. Preliminary analyses indicate that incorporation of these three features would lead to a mirror with under 5- $\mu$ rad slope error and with a maximum temperature of about $80{ }^{\circ} \mathrm{C}$ for a three-fold increase in incident power and power density. Stress levels in such a mirror will be high, and a prototype should be made and tested under simulated heat-load conditions.

\subsubsection{Other X-ray Optics Related Activities}

\section{Sagittal Focusing}

Sagittal focusing enhances the capabilities of a beamline through an increase in the flux density (photons $/ \mathrm{sec} / \mathrm{mm}^{2}$ ) delivered at the sample position. We have built and tested a sagittal bender for the second crystal of the cryogenic monochromator in the 1-ID beamline. The bender produces a 1:1 horizontal focus of the source at the 1-ID-C station $60 \mathrm{~m}$ from the $\mathrm{x}$-ray source. The bender mechanism is the same as the one that has been successfully implemented by SRI-CAT in the sector $1 \mathrm{BM}$ beamline and by UNI-CAT in the sector 33 ID beamline, with some modifications required to make it fit into the Kohzu monochromator tank in station 1-ID-A.

The bender accommodates a 114-mm-long by $40-\mathrm{mm}$-wide silicon (111) crystal. The 114-mm-long by 7-mm-wide region in the center of the crystal is $0.7 \mathrm{~mm}$ thick. On each side of the thin central web, the crystal is a least $10 \mathrm{~mm}$ thick. These thicker edges and the very long aspect ratio of the crystal act to suppress anticlastic bending of the diffracting portion of the crystal. The thin web is bent by the action of two PZTs, one at each end of the length of the crystal. A third PZT is located parallel to the long axis of the crystal and allows for cancellation of any twist introduced by the two curvature PZTs. The PZTs push against fixed micrometers, which are preloaded before installing the bender in the monochromator tank. The curvature PZTs have a maximum extension of 80 microns, which is adequate to focus the beam for energies up to $24 \mathrm{keV}$.

Tests were carried out on the mirror bender during the May 1998 run. To characterize the focus size, a $0.4-\mathrm{mm}$-wide horizontal slit was scanned through the beam. The slit was located in the 1-ID-C station, at $58.5 \mathrm{~m}$ from the center of the 1-ID straight section. The expected magnification for this geometry was 0.88. We tested the bender at 4 energies: 9, 12, 15, and $18 \mathrm{keV}$. Figure 5.26 shows the horizontal FWHM of the beam as function of the extension of the curvature PZTs for the $15-\mathrm{keV}$ case. The minimum focus was achieved at a PZT extension of 65 microns, giving a FWHM of $0.74 \mathrm{~mm}$. After deconvolving the slit size, the measured FWHM is $0.62 \mathrm{~mm}$, which is in good agreement with the expected value of $0.68 \mathrm{~mm}$. Figure 5.26 also shows the total intensity delivered by the sagittal crystal, $\mathrm{I}_{0}$, and the peak intensity through the 0.4-mmwide slits, $\mathrm{I}_{1}$. The peak flux through the slits 


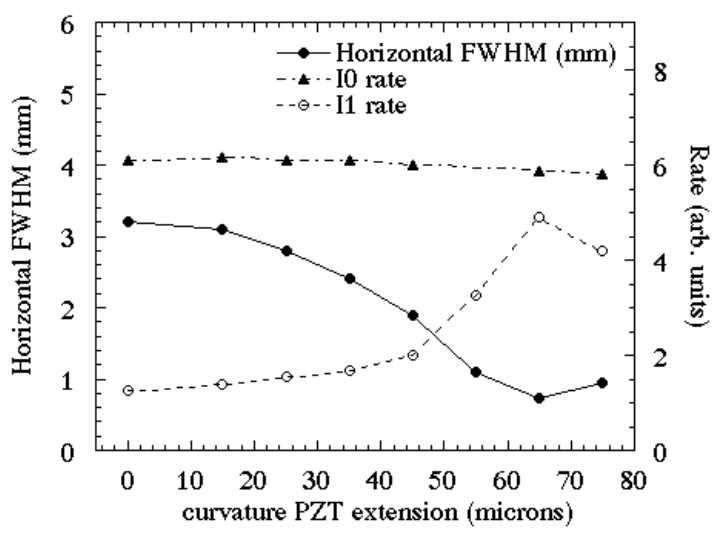

Fig. 5.26 Horizontal FWHM of the focused beam, total intensity 10 delivered by the bent crystal, and intensity II through the 0.4-mmwide scanning slit as a function of the extension of the curvature PZTs.

$I_{1}$ increased by a factor of 4 from unfocused beam ( 0 micron extension) to focused beam (65 micron extension). The expected intensity gain in $\mathrm{I}_{1}$ was approximately 4.8 , which is in reasonable agreement with the measurement. The total intensity $\mathrm{I}_{0}$ dropped by only $5 \%$, indicating that the performance of the bent crystal is not degraded as the curvature is increased. Rocking curve measurements of the silicon (333) reflection also indicated that bending of the crystal does not affect the diffraction performance.

We obtained similar results and agreement with theory at the four energies studied. The average horizontal FWHM at the focus was $0.65 \mathrm{~mm}$, while the average peak intensity gain through the 0.4-mm-wide slits was 3.9. The average loss in the flux delivered by the bent sagittal crystal was $7 \%$. These tests showed that the sagittally focusing crystal performs well over an extended energy range. A problem that remains to be addressed is the thermal stability of the crystal/bender assembly. When the undulator beam impinges on the cryogenically cooled first crystal, some of the incident power is scattered into the monochromator vacuum tank. A fraction of this scattered power is absorbed by the second-crystal assembly and results in thermal instability that alters the characteristics of the sagittal focus as a function of time. We plan to tackle this problem with a combined approach of shielding the second-crystal assembly to reduce the amount of absorbed power and of cooling or connecting the crystal/bender assembly to a heat sink.

\section{Compound Refractive Lenses}

The compound refractive lens is a relatively new addition to the growing list of $\mathrm{x}$-ray focusing optical components (Snigeriv et al., 1996). We have explored some of the potential uses for such a lens (Smither et al., 1997) and recently a prototype Be refractive lens was constructed for test purposes. The lens consisted of 50 hollow spheres machined into a Be substrate (Fig. 5.27). The web between each hollow sphere was $0.10 \mathrm{~mm}$ thick. The lens was made by machining hollow half spheres into two identical Be blocks and placing them together to make the row of hollow spheres. The lens was tested at the APS on the 1-ID beamline with a $10-\mathrm{keV}$ monochromatic beam. The transmission at $10 \mathrm{keV}$ is about $16 \%$, which means that $16 \%$ of the flux on a 1-mm-diameter spot at the entrance to the lens emerged from the end of the lens. The focal spot remained slightly less than 40 microns for focal distances of 1400 to $1300 \mathrm{~mm}$. The narrowest focus came at a distance of $1356 \mathrm{~mm}$ behind the lens, when the initial $x$-ray beam was collimated down to a square $0.4 \mathrm{~mm} \times 0.4 \mathrm{~mm}$ and the lens was well aligned. This arrangement gave a focal spot of 33.6 microns. The lens 


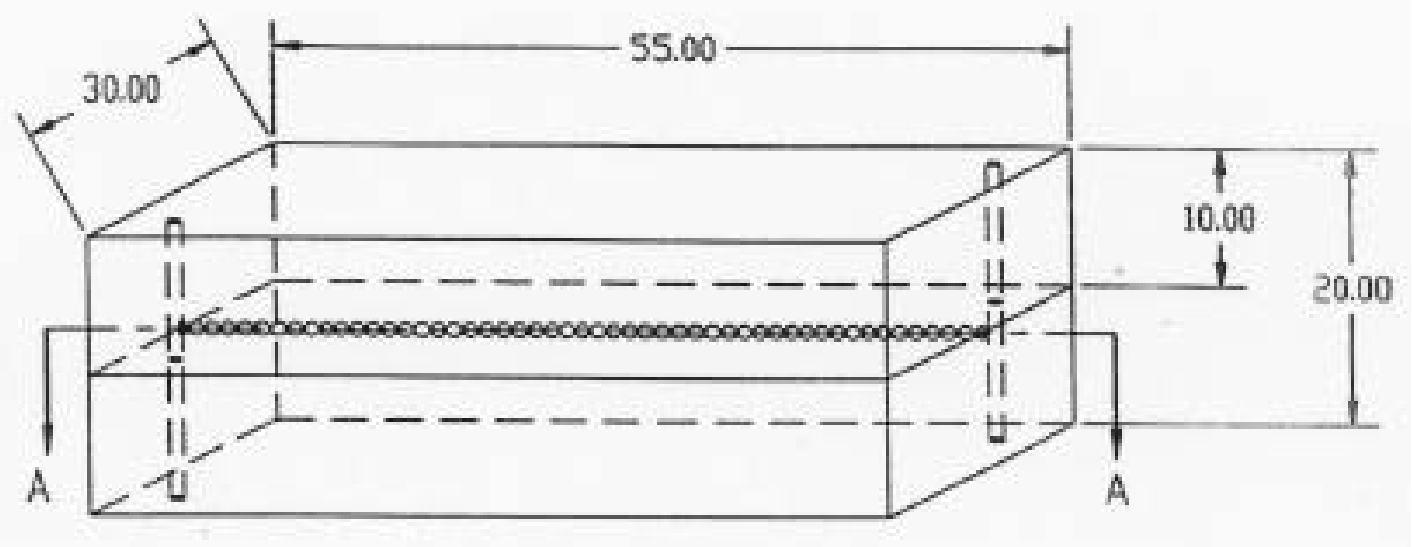

Fig. 5.27 Schematic of the Be refractive $x$-ray lens. It consists of 50 1-mm-diameter hollow spheres in a beryllium substrate. The web between each hollow sphere is 0.1 mm thick. All dimensions are in $\mathrm{mm}$.

transmission for this smaller beam is approximately $30 \%$. The theoretical value for the focal spot diameter was less than 10 microns. The wider spot diameter is believed to be due partly to the spherical shape of the hollow cavities, which causes focusing abberations. This effect focuses the $\mathrm{x}$-rays farther from the optical axis at shorter distances from the lens than the $\mathrm{x}$-rays close to the optical axis of the lens, which was manifested through the best focus coming at a distance $100 \mathrm{~cm}$ shorter than the theoretical focal length of $1.466 \mathrm{~m}$. Part of this shortening of the focal length could also be due to a slightly smaller diameter of the hollow spheres. The second factor that broadens the focal spot is the unevenness of the machining of the hollow sphere. This roughness was apparent when the halfhollow spheres were examined under a microscope. A method has been devised to eliminate the ridges in the surface of the hollow spheres and was tested on an aluminum lens (20 1-mm-diameter hollow spheres). The procedure consists of smoothing the hollow sphere surfaces using a special tool with a small amount of very fine polishing compound present while the lens is submerged in water. This tool was also used to thin the wall between the hollow spheres of the $\mathrm{Al}$ lens, from $0.2 \mathrm{~mm}$ to $0.1 \mathrm{~mm}$. This change improved the transmission of the aluminum lens by a factor of 5 at $30 \mathrm{keV}$.

\section{Gradient d-Spacing Crystals (Ge/Si and $\mathrm{Si} / \mathrm{Ge}$ )}

The width of the energy band diffracted by a perfect crystal is determined by the Darwin width of the crystal and/or by the divergence of the beam incident on the crystal. The energy spread due to the beam divergence can be eliminated by using a crystal whose $\mathrm{d}$-spacing varies along the footprint of the incident beam such that the varying d-spacing compensates for the varying incident angles. Recently it has become possible to grow near-perfect crystals of Ge-Si mixtures, the d-spacing of which can be changed by varying the relative concentrations of the two components. Tests were performed on both single crystals of germanium with a small, varying 
concentration of silicon and single crystals of silicon with a varying concentration of germanium grown by Nikolai Abrosimov at the Institute for Crystal Growth, Berlin. Both rocking curves and d-spacings were measured. The concentration of $\mathrm{Si}$ in the $\mathrm{Ge} / \mathrm{Si}$ crystals varied from $0.1 \%$ to $2.6 \%$, which changed the d-spacing by $0.104 \%$. The rocking curves varied from 5 to 20 arc $\mathrm{sec}$ in the low $\mathrm{Si}$ region (less than $1 \% \mathrm{Si}$ ) and from 20 to 200 arc sec in the high $\mathrm{Si}$ region (greater than $1 \% \mathrm{Si}$ ). The concentration of $\mathrm{Ge}$ in the two $\mathrm{Si} / \mathrm{Ge}$ crystals was $3.0 \%$ and $3.9 \%$. The rocking curves (FWHM) varied from 40 arc sec to 50 arc sec in the $3.9 \%$ Ge crystal and from 60 to 120 arc sec in the $3.0 \%$ Si crystal. Thus the rocking curves appear to be more sensitive to the parameters and conditions used during the growing of these crystals than to the concentration of Si. A 4\% change in the concentration of Ge will change the dspacings by $0.16 \%$. This would correspond to $67 \mathrm{arc} \mathrm{sec}$ at $10 \mathrm{keV}$ for the [111] planes in silicon. At $30 \mathrm{~m}$, this corresponds to a vertical height of $10 \mathrm{~mm}$; thus a change in concentration of $1 \%$ or less will be sufficient for most synchrotron applications. Crystals with concentration changes of $\mathrm{Ge}$ of $1 \%$ or less have been grown with mosaic structure widths of a few arc sec and, thus, should be useful in synchrotron experiments.

\section{Crystal Lenses for Medical Imaging}

A short focal length crystal diffraction lens has been developed for medical imaging applications. The lens is designed to focus the $141-\mathrm{keV}$ gamma ray from $99 \mathrm{mTc}$, which is injected into the blood stream of cancer patients. Fast growing cancer cells will incorporate more of the radioactivity than normal cells. A full body scan is then made to locate possible cancer sites in the body.
The new medical lens will be used to verify the presence of these enhanced sites and obtain a 3-D image of the cancer. This 3D image will greatly simplify the taking of tissue samples and help guide subsequent surgery. The lens system will also be able to reject those borderline indications in the full body scan that are false and eliminate unnecessary tissue sampling. The lens is constructed of small copper crystals $4 \mathrm{~mm}$ by $4 \mathrm{~mm}$ on a side and 2 to $3 \mathrm{~mm}$ thick, mounted in rings such that each ring uses a different set of crystalline planes to diffract and focus the gamma rays. The focal length of this lens for the $141-\mathrm{keV} 99 \mathrm{mTc}$ line is 50 $\mathrm{cm}$. The patient will be located $100 \mathrm{~cm}$ (two focal lengths) from the lens, and the detector will be located $100 \mathrm{~cm}$ behind the lens. Scanning on and off the cancer site will be done by moving the patient.

\section{Crystal Lenses for Astrophysical Applications}

An international collaboration (U.S., France, England, Italy, and Germany) has been formed to perform a series of astrophysics balloon experiments that use a crystal diffraction lens to look at gamma rays from distant super nova remnants and other distant astrophysics sources. The lens will use $\mathrm{Ge}$ and $\mathrm{Ge} / \mathrm{Si}$ crystals and will be constructed at the CESR laboratory in Toulouse, France. Argonne has supplied the Ti-Al alloy lens frame and support structure, as well as the design and technology needed to mount and align the lens crystals. Argonne will also be supporting this effort by making measurement of the diffraction efficiency of different mosaic crystals. Measurements have been made on crystals of $\mathrm{Ge}, \mathrm{Ge} / \mathrm{Si}$, and $\mathrm{Cu}$. The first samples of Mo and W crystals are expected in Nov. 1998. 


\subsection{New Instruments and Techniques}

\subsubsection{Introduction}

The development of beamline instrumentation and techniques that demonstrate and enhance the unique opportunities that are available at the Advanced Photon Source is undertaken primarily by XFD scientists and engineers working on the Synchrotron Radiation Instrumentation Collaborative Access Team (SRI-CAT) beamlines. As the facility has matured, the role of SRI-CAT has also evolved as reflected in our recently updated mission statement:

- To conduct R\&D activities towards the improvement of IDs, standard components, high-heat-load optics, and other novel $\mathrm{x}$-ray optical components and to develop innovative techniques useful to the entire community of APS CATs.

- To develop and implement strategic instrumentation programs that will open up new areas of research at the APS

- To attract, educate, and foster new research communities in the uses for and applications of synchrotron radiation.

Testing of beamline components and $\mathrm{x}$-ray optics and the development of novel experimental techniques primarily take place on those SRI-CAT beamlines that were designed with flexibility in mind. The beamlines designed to carry out specific areas of technique development, called strategic instruments, have seen an increase in user demand as they have proceeded from the commissioning to the operational phase over the last year. Construction has begun on sector 4, for another strategic instrumentation program, namely the generation and use of variably polarized $\mathrm{x}$ rays over the energy range of 0.5 to 100 $\mathrm{keV}$. The sector 4-ID beamline will join those of sectors 2 and 3 to make up the complete set of the SRI-CAT strategic instrumentation programs:

- a milli-electron volt resolution instrument

- a micro-to-nano-electron volt instrument to perform nuclear resonant scattering experiments

- a 1-4 keV radiation source and instrumentation for use of coherent soft x-rays

- instrumentation for development of hard x-ray microfocusing optics and techniques

- a $0.5 \mathrm{keV}$ to $100 \mathrm{keV}$ variable polarization beamline

A schematic of the locations of these programs, along with the other major programs on the SRI-CAT beamlines, is shown in Fig. 5.28. Also indicated in Fig. 5.28 is a backscattering beamline (see section 5.2.3 for details). Because of the location of the 1-ID straight section with respect to other beamlines (i.e., there are no other beamlines immediately upstream), this beamline affords a unique opportunity for the development of a backscattering 


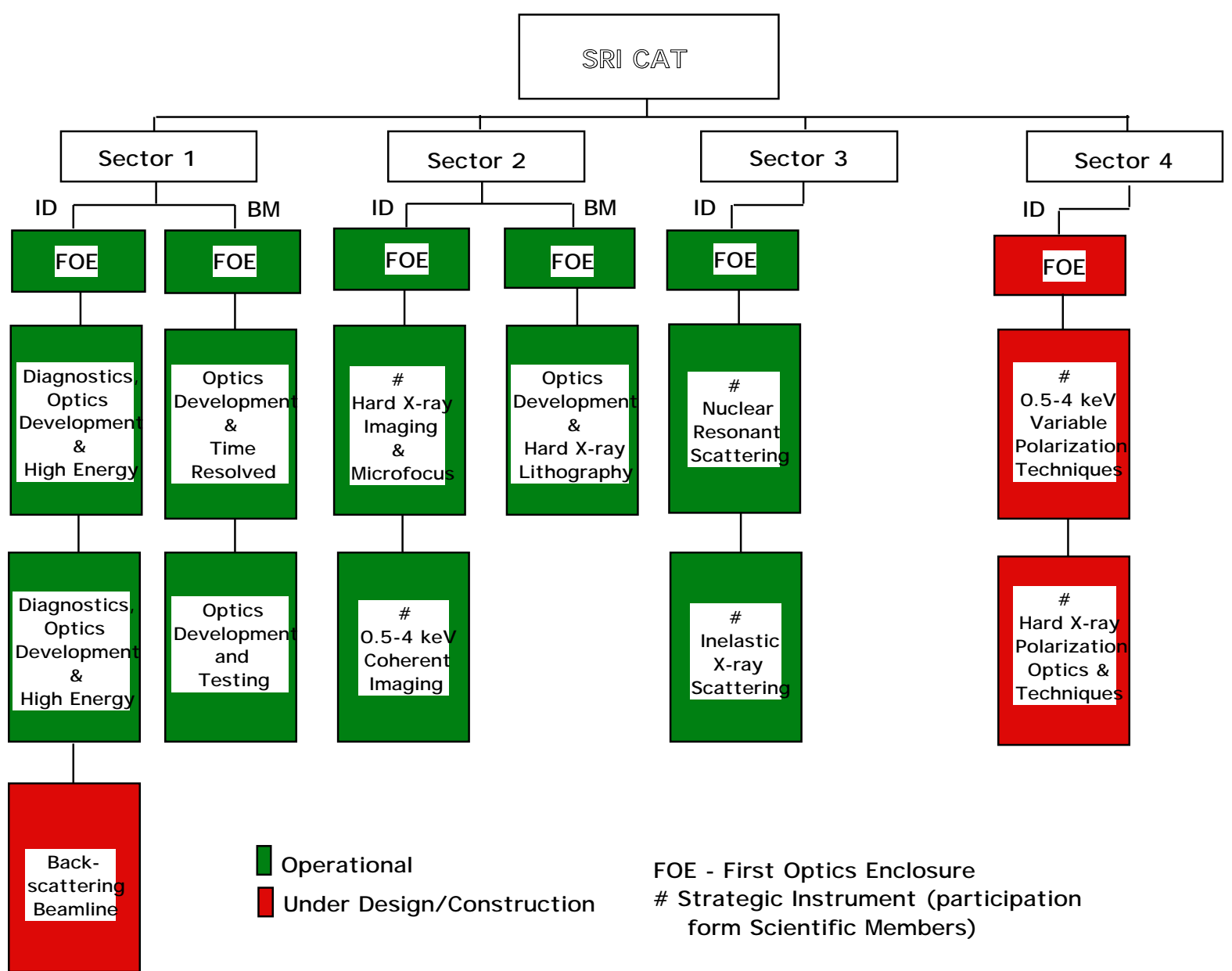

Fig. 5.28 Schematic of SRI-CAT showing activities and locations of various programs on the six beamlines.

beamline $\left(2 \Theta_{\mathrm{B}}=180^{\circ}\right)$ by allowing the diffracted beam to pass back through the ID straight section and to emerge out the back end of the ID (see Fig. 5.12). Over the last year, the storage ring was fitted with a new vacuum chamber by ASD staff that would allow the backscattered beam to exit the vacuum chamber. A "back-end" was recently installed from the vacuum chamber port to a port through the storage ring tunnel. Development of the monochromator, which will be installed in 1-ID $\mathrm{A} / \mathrm{B}$, and experiment station, which will be located in the equipment assembly area, is currently underway. This beamline will allow a detailed study of the physics of diffraction at exact backscattering geometry in addition to providing a beam of high resolution and large longitudinal coherence length in a nearly background-free environment.

The SRI-CAT staff have been active in making new scientific communities aware of the potential of the APS. One area to which the SRI-CAT beams have been put to use is the study of a variety of antiquities. Researchers from The Oriental Institute at The University of Chicago, the Field 
Museum, and the Adler Planetarium (the latter two are both also located in Chicago, IL) have collaborated with the SRI-CAT staff in several feasibility studies to explore the potential application of synchrotron radiation to metallurgical and other studies relevant to archaeological and historical research.

\subsubsection{Sector 1}

\section{1-BM Beamline and Optics}

Numerous experiments have been conducted on beamline 1-BM starting with the observation of first light in March of 1995. Recently all the optical components have been put into place to permit operation of this beamline as originally designed. In many ways the optics on the 1-BM beamline are more complicated than those on some of the ID beamlines. The reason for this increased level of complication is that efficient use of a bending magnet beam absolutely requires focusing of the beam. In the following section, we summarize some of the commissioning activities that have occurred during the past year for the major optical components of the 1-BM beamline. In particular, we concentrate on the properties of the doubly focused beam entering the 1-BM-C station.

The first major optical component on the beamline is a water-cooled 1.2-m-long palladium-coated mirror. This flat mirror, bendable to a cylindrical shape, is used to vertically collimate the beam (i.e., focus the beam at infinity). Collimating the beam allows the user to accept a larger vertical beam without sacrificing energy resolution, because all the rays in the beam after the mirror will make an identical angle with any monochromator crystal further downstream. Commissioning activities associated with this mirror were concentrated on the measurement of the decrease in the energy resolution, $\Delta \mathrm{E} / \mathrm{E}$. These measurements were made using a highly dispersive $\mathrm{Si}$ analyzer crystal in the beam after the monochromator to monitor $\Delta \mathrm{E} / \mathrm{E}$ as the mirror figure was changed from flat to collimating. Measurements at $10 \mathrm{keV}$ showed that the bandwidth passed by the monochromator for a $2.5-\mathrm{mm}$ vertical beam $22.9 \mathrm{~m}$ from the source decreased from $5.5 \times 10^{-4}$ to $1.5 \times 10^{-4}$ for the $\mathrm{Si}$ (111) reflection and from $3.6 \times 10^{-4}$ to $8.1 \times 10^{-5}$ for $\mathrm{Si}(220)$. The lower values on the energy resolution are only $15 \%$ and $25 \%$ that expected for perfect collimation. The discrepancy can likely be accounted for by residual fabrication slope errors in the mirror. Later measurements demonstrated that, while the central $0.8 \mathrm{~m}$ of the mirror had only 2-3 microrad deviations from an ideal bend, both of the edges of the mirror were substantially overbent to 10 microrads. Thus by using a smaller beam the theoretical resolution can be approached, albeit with a loss in flux.

The collimating mirror is followed by the double-crystal monochromator (DCM). This monochromator has been used quite extensively over the past year but has recently been improved by the installation of a sagittally bent second crystal, which provides horizontal focusing of the beam into the $\mathrm{C}$-station. Initial tests of this sagittal focusing crystal, designed following the "golden ratio" concept put forth by Kushnir et al. (1993), have been quite promising. A horizontal focus of $0.45 \mathrm{~mm}$ was achieved at $10 \mathrm{keV}$ for a 72-mm-wide beam incident on the monochromator using the Si (220) reflection. Furthermore, this focal size was achieved with a broadening of the 
monochromator crystal rocking curve only $25 \%$ above that of the ideal case for a flat crystal.

The third and final major optical component in the beamline is a 1-m-long, palladiumcoated mirror located between the B- and C-stations. This mirror, also a flat mirror bendable to a cylinder, provides vertical focusing of the beam for the C-station. The measured vertical size of the beam from this mirror was 108 microns, which is only $20 \%$ above an ideal image expected from ray tracing the source. Although we were able to achieve a good focus with this mirror, a great deal of structure (horizontal striations) was observed in the unfocused beam. After several tests, it was determined that the structure arose from 2-3 microradian fabrication slope errors. One should note that, for a mirror of this size, slope errors in this range are at the limit of current mirror polishing technology. Although the collimating mirror on the beamline did not display structure as dramatic as this, several other mirrors at APS beamlines have exhibited similar features. Features such as these are much more readily observable at the APS, as opposed to at other synchrotrons, due to the small source size coupled with the availability of long beamlines at the APS.

A summary of the combined performance of all the 1-BM optics is contained in Fig. 5.29 and Table 5.5. Figure 5.29 shows a doubly focused $9.0 \mathrm{keV}$ beam in the C-station using a Si (111) monochromator. This focal spot size was attained using a $60 \times 2.5 \mathrm{~mm}^{2}$ beam incident on the monochromator. Profiles of this image give full-width halfmaximum (FWHM) values of $0.25 \mathrm{~mm}$ vertical and $0.60 \mathrm{~mm}$ horizontal, which are roughly twice as large as expected for ideal

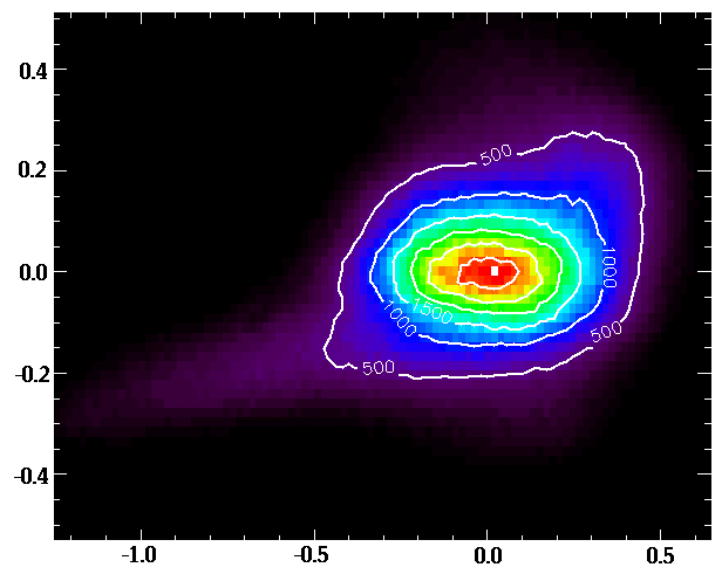

Fig. 5.29 Doubly focused beam in the 1-BM-C station with a $72 \times 2.5 \mathrm{~mm}^{2}$ beam incident on the sagittally focusing Si (111) monochromator.

optics. The increase in the vertical spot size compared to that taken with flat crystal optics is believed to be due to aberrations induced by a slight twisting in the sagittal crystal. While the increase in the horizontal spot size is probably due to nonuniformities in the thickness of the bending crystal. New crystals are currently being fabricated to improve the focusing qualities. Table 5.5 lists the achieved photon fluxes and bandwidths into the C-station when using a $50 \times 2.5 \mathrm{~mm}^{2}$ beam, $22.9 \mathrm{~m}$ from the source, and it gives the percentage of these values compared to the ideal case of perfect optics.

It should be pointed out that the flux value measured on beamline 1-BM is within a factor of 10 of the flux obtained from an unfocused ID beam. Furthermore, because of the collimation of the beam prior to the monochromator, the bandwidths on 1-BM are nearly the same as those seen on the ID beamlines. This demonstrates that, by properly designing the optics on a bending magnet source at the APS and for experiments that require only a large 
Table 5.5 Photon Fluxes and Bandwidths Achieved in the 1-BM-C Station.

\begin{tabular}{|c|c|c|c|c|}
\hline Mono Crystal & $\begin{array}{c}\text { Flux } \\
(\mathrm{ph} / \mathrm{s} / 100 \mathrm{~mA})\end{array}$ & $\begin{array}{c}\text { Percentage } \\
\text { of Ideal }\end{array}$ & $\Delta \mathrm{E} / \mathrm{E}$ & $\begin{array}{c}\text { Percentage } \\
\text { of Ideal }\end{array}$ \\
\hline $\mathrm{Si}(111)(9 \mathrm{keV})$ & $1.3 \times 10^{12}$ & $72 \%$ & $1.5 \times 10^{-4}$ & $115 \%$ \\
\hline $\mathrm{Si}(220)(10 \mathrm{keV})$ & $5.0 \times 10^{11}$ & $65 \%$ & $8.1 \times 10^{-5}$ & $125 \%$ \\
\hline
\end{tabular}

incident flux on the sample with relaxed conditions on beam brilliance, a BM beamline can be a viable alternative to an ID beamline. As of July 1, 1998, this beamline has been assigned operational status.

\section{1-ID Beamline and Optics}

The 1-ID beamline is fully commissioned and has been operating with users (including independent investigators) throughout the last year. The only major change to the beamline configuration is that L1 whitebeam slits were installed. Previously, temporary white-beam slits were used. The installation of the L1 slits completes the installation of standard components for 1-ID.

An 8-circle Huber diffractometer has been temporarily installed in the 1-ID-C experiment station. This diffractometer was purchased for sector 4 and will be moved when that beamline is ready for operations, but until then it will be used on 1-ID. The addition of the 8-circle (replacing the standard 6-circle) diffractometer greatly increases the capability of the beamline to study off-specular reflectivity and other outof-plane scattering phenomena.

The 1-ID beamline is, in part, a test bed for optics development. Installation and testing of crystals and other optical components has been a frequent occurrence over the past year and will continue into the foreseeable future. During the past year, 1-ID has had three different optical configurations. For experiments in the 6-18 keV range, $\mathrm{Si}$ (111) crystals are installed into the Kohzu DCM. For the energy range of 9-45 keV, Si (311) crystals are installed into the Kohzu DCM. In addition to giving the higher energy range, this configuration has an energy bandpass roughly $20 \%$ of the $\mathrm{Si}$ (111) (with corresponding reduction in intensity), which is particularly useful for studies of anomalous diffraction for elements with $\mathrm{K}$-edges in the 20-45 keV range. The final configuration is for experiments requiring photons in the $40-150 \mathrm{keV}$ region. For this energy range, an auxillary monochromator is installed in 1-ID-B. The first crystal in this monochromator is cooled with liquid nitrogen and resides inside a large vacuum chamber. The second crystal is not cooled and is placed outside the vacuum chamber. This arrangement allows for large crystal separations (up to a meter) and, as a consequence, the standard $35-\mathrm{mm}$ offset between white and monochromatic beam can be obtained for energies up to $150 \mathrm{keV}$. With such an offset, the high-energy monochromatic beam can be brought into 1-ID-C allowing for very low background levels as compared with working in the same station with the monochromator. 
After several years of operation cooling the silicon monochromators with liquid nitrogen, we are pleased with the performance of this system (Kohzu monochromator, crystals, liquid nitrogen pump, etc.). Nonetheless, two improvements for the monochromator were tested on the 1-ID line last year: cooling the second crystal to reduce beam motion as a function of energy and adding a sagittally focusing second crystal. (Both these activities are described in more detail in section 5.3.)

In collaboration with the sector 2 microfocusing effort, zone plates for focusing at $40 \mathrm{keV}$ were tested at 1-ID. The zone plates used for this study were designed to produce a $\pi$ phase shift at $20 \mathrm{keV}$ (i.e., $\pi / 2$ at $40 \mathrm{keV}$ ). They were made of gold with a thickness of 3.3 microns, had an outer zone width of 0.3 microns, an overall diameter of 80 microns, and a focusing distance of $1 \mathrm{~m}$ (at $40 \mathrm{keV}$ ). From one zone plate, a focus beam size of 2.5 microns by 8 microns (vertically and horizontally, respectively) was achieved with an efficiency of $19 \%$. This is essentially the theoretical efficiency for the system tested. To increase the efficiency, a second zone plate was "stacked" with the first to give a total of $\pi$ phase shift at $40 \mathrm{keV}$. The efficiency of this system was measured to be $38 \%$, showing that the two zone plates could be aligned well enough to add in phase with each other. The total flux density gain of the stacked zone plates was a factor of 120 .

\section{Hard X-ray Polarization Program}

The objective of this program is to develop instrumentation and techniques suitable for performing investigations of magnetic systems. During the previous years, effort was directed toward evaluating phaseretarding crystal optics. These accomplishments were described in the XFD Progress Report 1996-97. During 1997-98, the focus has been to demonstrate unique magnetic measurements using the phaseretarding crystal optics. In the following, some of the early results from these scientific research studies are described.

A study of the magnetic properties of $\mathrm{Fe} / \mathrm{Gd}$ multilayers has been carried out during several runs on beamline 1-ID. These studies began as an investigation of the spin structures of the $\mathrm{Fe}$ and $\mathrm{Gd}$ layers as a function of temperature and applied magnetic field. More recently, the studies have been expanded to include measurements of magnetic roughness of the multilayers by using spin-sensitive diffuse scattering.

The Fe/Gd multilayers are fundamentally an exciting system to study because they exhibit a variety of spin structures, as can be seen in the temperature-field phase diagram of Fig. 5.30 (LePage and Camley, 1990). The $\mathrm{Fe}$ and $\mathrm{Gd}$ layers couple antiferromagnetically and, at low applied fields, are Gd-aligned (at low temperatures where the $\mathrm{Gd}$ moment is dominant) or Fealigned (above the compensation temperature where the $\mathrm{Fe}$ moment is dominant). Due to the competition between the Zeeman and exchange energies, there are also bulk- and surface-twisted phases for larger applied magnetic fields. In addition to the rich phase diagram, the existence of surface and bulk magnetic structures makes the $\mathrm{Fe} / \mathrm{Gd}$ multilayers an excellent model system to study surface magnetic reconstruction problems, i.e., how a spin configuration at the surface may differ from the bulk spin configuration. This is analogous to the crystal surface 


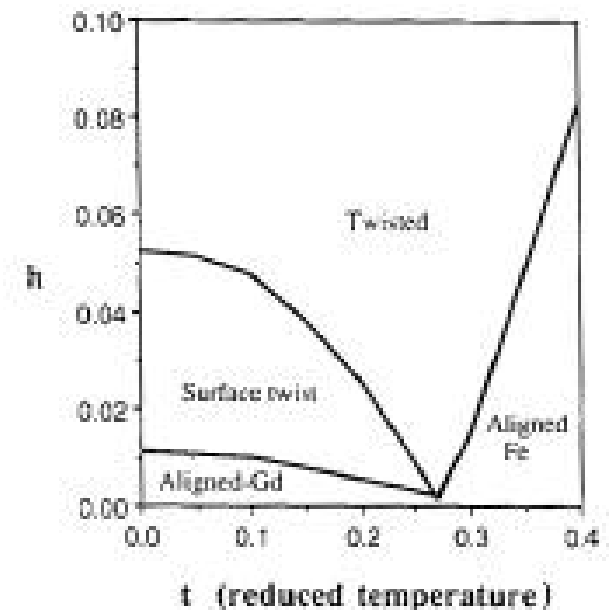

Fig. 5.30 Calculated phase diagram for a Fe/Gd multilayer. The axes are in units of reduced temperature (T/TC for TC of $\mathrm{Fe}$ ) and field (H/JSFe). [Reprinted with permission from LePage and Camley, Phys. Rev. Lett. 65 (1990) 1152.]

reconstruction that has been widely studied in recent years.

The Fe/Gd multilayers are also a useful system for studies of magnetic roughness-including vertical and in-plane correlation lengths - in multilayers. This is an extremely important issue in technological applications in which spin transport effects are utilized (e.g., the gian magneto-resistance effect) because the size of the effect is directly related to the inplane magnetic roughness. Magnetic roughness measurements to date have been confined to transition metal samples consisting of no more than a few layers because of the necessity of using low-energy $\mathrm{x}$-rays for resonant enhancement at transition metal $\mathrm{L}$ edges. But by using a rare-earth sample (with L edges in the hard $\mathrm{x}$-ray regime) and high-intensity undulator radiation, the entire multilayer can be probed.
Both the spin structure and magnetic roughness were studied using resonant $\mathrm{x}$-ray magnetic scattering - at the $\mathrm{Gd} \mathrm{L}_{3}$ and $\mathrm{Fe} \mathrm{K}$ edges for the spin structure measurements and at the $\mathrm{Gd} \mathrm{L}_{3}$ edge for the magnetic roughness measurements. The magnetic information was extracted from specular reflectivity and diffuse scattering measurements by subtracting data sets that differ only by having opposite photon helicities. A diamond (111) phase retarder produced the required circularly polarized photons, and the helicity was switched by rotating the retarder by a fixed amount to either side of the Bragg peak. The sample was placed between the poles of a permanent magnet, and the sample and magnet were mounted in a closed-cycle helium refrigerator in order to explore a range of sample temperatures.

A comprehensive set of spin structure measurements has been completed for an applied field of $2.4 \mathrm{kG}$. Due to the fact that resonant $\mathrm{x}$-ray magnetic scattering is only sensitive to magnetization in the scattering plane, data were collected with two relative orientations of the field-parallel and perpendicular to the scattering plane-at each temperature in order to differentiate between aligned and twisted phases. The difference signal for the perpendicular orientation was observed to disappear in the temperature range of 160-180 K (see Fig. 5.31), indicating a phase transition from twisted to aligned. One other notable feature of the data was the sign change of the difference signal as a function of superlattice Bragg peak, which was seen at the $\mathrm{Gd} \mathrm{L}_{3}$ edge but not at the $\mathrm{Fe} \mathrm{K}$ edge. From simulations, such sign flipping can be accounted for by nonuniformity of the $\mathrm{Gd}$ moment within a given layer. This explanation also agrees with theoretical calculations (Camley, 1989) in which 

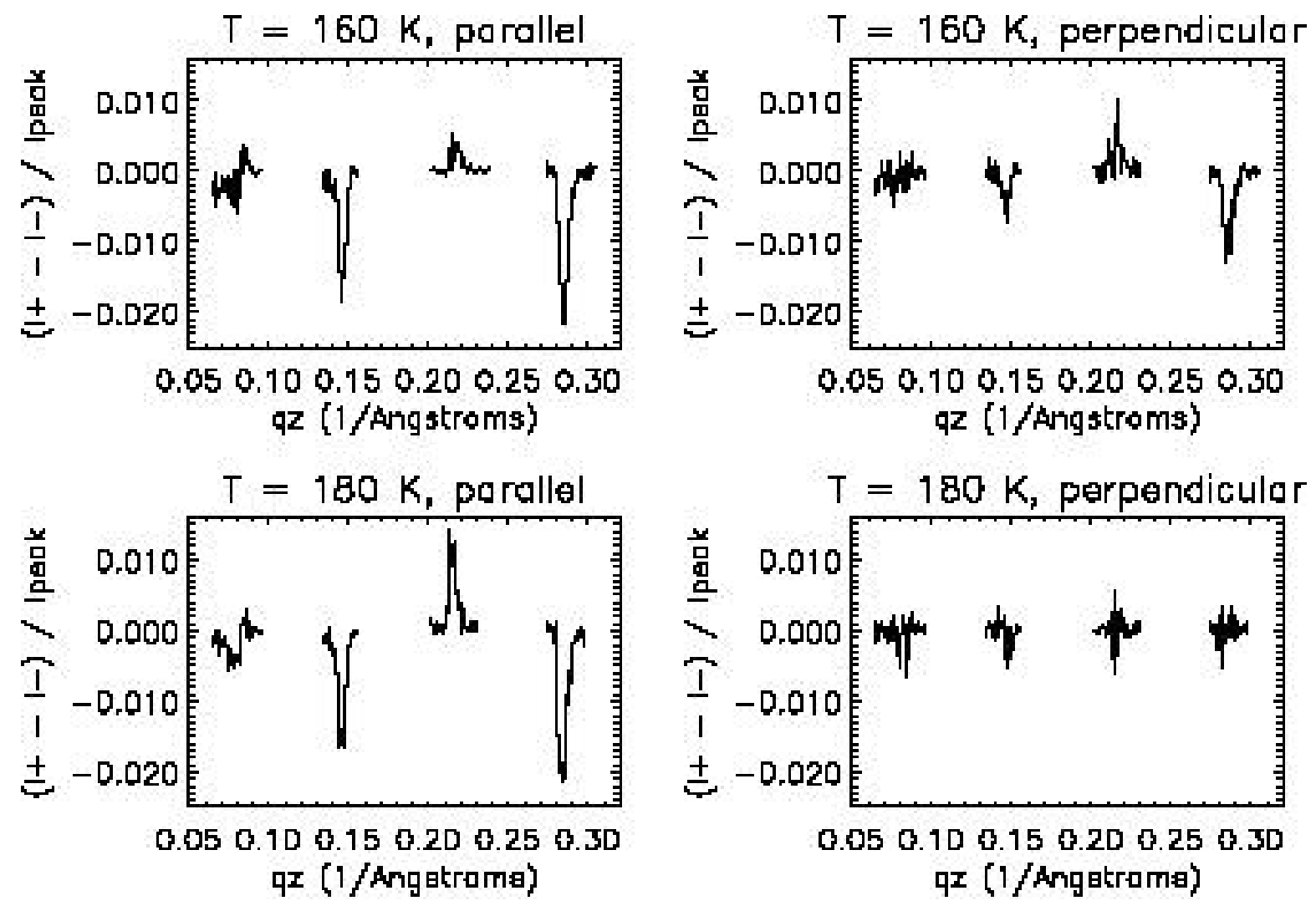

Fig. 5.31 Magnetic reflectivity data collected at the $G d L_{3}$ edge at temperatures of $160 \mathrm{~K}$ and $180 \mathrm{~K}$. The difference signal between the reflectivity measured with opposite photon helicities divided by the summed signal at the superlattice Bragg peak is plotted. Note the disappearance of the difference signal for the perpendicular relative orientation (i.e., the field is applied perpendicular to the scattering plane) at $180 \mathrm{~K}$, indicating that the multilayer is in an aligned phase.

interface coupling of the $\mathrm{Fe}$ and $\mathrm{Gd}$ atoms results in either an enhanced or reduced $\mathrm{Gd}$ moment at the interface as compared to in the bulk of the layer, depending upon the strength of the interface coupling. In contrast, a lack of sign flipping in the data collected at the $\mathrm{Fe} \mathrm{K}$ edge suggests uniformity of the $\mathrm{Fe}$ moment within each layer.

Spin-sensitive magnetic diffuse scattering measurements have been made for an Fe-aligned phase of the $\mathrm{Fe} / \mathrm{Gd}$ multilayer in an applied field of $3.4 \mathrm{kG}$. Magnetic diffuse scattering is a very weak process, so reasonable statistics have only been collected for two transverse scans (which give information about in-plane magnetic correlations) and an offset scan in which the sample was rotated 0.12 degrees away from specular (which gives information about out-of-plane magnetic correlations). These three data sets are extremely rich-even showing features in the magnetic signal corresponding in reciprocal space to multiple scattering events in the charge scattering-and, for a complete analysis, 
will require an extension of the distorted wave Born approximation into the resonant magnetic regime.

The polarization studies have also included work on $\mathrm{x}$-ray magnetic circular dichroism (XMCD) in $\mathrm{REFe}_{2}$ compounds $(\mathrm{RE}=$ rare earth) as part of an ongoing study of the temperature dependence of XMCD in rare earth-transition metal materials. The goal of these measurements has been to measure the different magnetic exchange couplings in these compounds and plot their variation with temperature. An understanding of the exchange couplings that control the magnetization of the $5 \mathrm{~d}$ band is essential in the development of new magnetic materials. The strength of the exchange couplings can be extracted from XMCD spectra by measuring the variation of the features in the dichroic spectra with temperature. Each feature, in principle, can be correlated with a specific electronic orbital state in the compound. Figure 5.32 shows a measurement of two such spectra taken at the $\mathrm{Tm} \mathrm{L}_{3}$ edge of $\mathrm{TmFe}_{2}$. The sign of the XMCD spectra is inverted upon raising the temperature because the material goes through the compensation point where the dominant magnetization sublattice of the

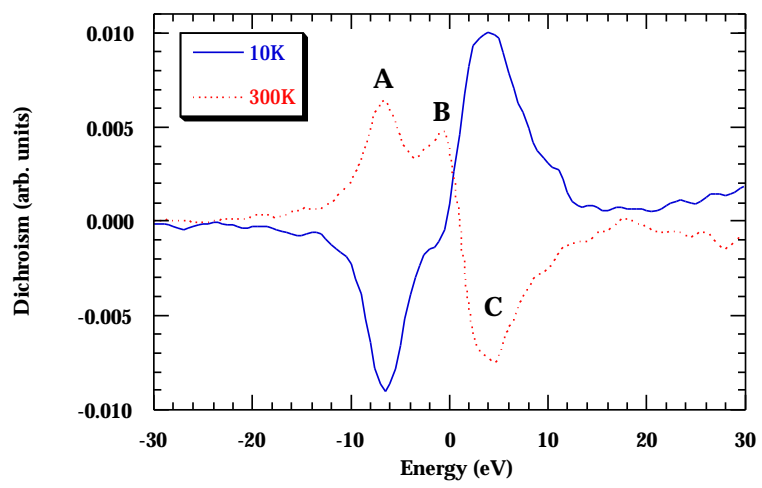

Fig. 5.32 Dichroism signal measured at the $\mathrm{Tm} \mathrm{L}_{3}$ edge of $\mathrm{TmFe}_{2}$ at $10 \mathrm{~K}$ and $300 \mathrm{~K}$. sample changes from $\mathrm{Tm}$ to $\mathrm{Fe}$. $\mathrm{Tm}$ is an interesting compound, since in addition to features $\mathrm{A}$ and $\mathrm{C}$, which we showed in a previous study to arise from quadrupolar and dipolar transitions to rare earth $4 \mathrm{f}$ and $5 \mathrm{~d}$ states, respectively, it possesses a feature (B) that becomes larger with temperature. The origin of this feature, which is not as prominent in other rare earths in the $\mathrm{REFe}_{2}$ series, is still uncertain, but our study suggests that it might arise from a hybridization of $\mathrm{Tm} 5 \mathrm{~d}$ states with the Fe $3 \mathrm{~d}$ states.

These measurements utilized the diamond phase-retarding optics developed here at the APS. The use of these optics has proved essential for these types of experiments due to the difficulty of reversing the sample magnetization in cryogenic environments.

The sample investigations of magnetic systems reflect a need for a dedicated beamline for such studies utilizing x-ray polarization techniques. This need has led SRI-CAT to plan sector 4, which is dedicated to the development of new polarization instruments and techniques.

\section{Time-Resolved Program}

As part of the time-resolved program in XFD, we are developing three classes of high-speed x-ray beam choppers or shutters. These beam choppers will span ten decades of time resolution and will offer a variable time between pulses.

The first class of beam chopper is based upon a commercial solenoid operating as a linear shutter. This design will cover the open time window range from a second to less than 1 millisecond. The desired 
operating parameters are two fold. First, a linear motion of the solenoid plunger, which can open or close an aperture quickly, on the order of 1 to $2 \mathrm{~ms}$, and remain open or closed for a period varying from $20 \mathrm{~ms}$ to a second. The maximum operation rate would be $50 \mathrm{~Hz}$. Second, we want a system that can open and close the time window on the order of 300 microseconds and remain closed for an unspecified time. Both of these designs depend upon the driving power supply. A pulsed high-voltage $(400 \mathrm{~V})$ high-current (5 A) power supply is being developed in house to drive the solenoid beam choppers. They will be used with the charge coupled device (CCD) camera detectors and high-speed beam chopper described below.

The next class of beam chopper is also based upon a commercial product, namely a highspeed line scanner. The central component for this device is a high-speed (80000 RPM) rotating beryllium cylinder with a narrow slot through the center that can rotate synchronously with respect to the orbital frequency of the storage ring. The large numerical aperture of the slot forms a fixed open time window of about 2.65 microseconds. (The close time is about 300 microseconds, hence the need for the fast solenoid shutter described above for applications requiring a longer close time.) Pulses shorter than 2.65 microseconds would require selective loading patterns of the APS storage ring, but in general, $x$-ray pulses as short as 75 psec, corresponding to a single bunch of stored particles, could be achieved with this synchronously operated beam chopper. Delivery of this item is expected in the fall of 1998.

The final class of beam chopper consists of a rotating $\mathrm{Si}$ crystal cube. In the normal fill pattern of the APS storage ring, about two thirds of the ring is filled, usually with about 25 bunches. The time between these bunches is typically about $100-150 \mathrm{~ns}$ (depends on the exact fill pattern). For certain time-resolved experiments, the ability to change this time structure is desirable. In particular, a longer inter-bunch time may be useful. With a rotating crystal, we have demonstrated that it is possible to increase the time between $\mathrm{x}$-ray bursts. By using a $15-\mathrm{mm}$ silicon cube mounted on a Stanford Research Instruments SR 540 beam chopper motor, we were able to take advantage of the small Darwin width opening angle of the crystal as a fast shutter for a monochromatic beam. The crystal was cut so that there were four Si (220) reflecting surfaces available. Depending on the speed of the crystal rotation, monochromatic $\mathrm{x}$-ray bursts with varying interburst times from about 1 second to 250 msec were achieved. In the first case, the length of the x-ray burst is actually the entire fill pattern of the synchrotron ring $(\sim 2.5 \mu \mathrm{sec})$; while in the latter case, the length of the $\mathrm{x}$-ray burst is actually one individual pulse $(\sim 100 \mathrm{ps})$ from the fill pattern. In this initial demonstration, the rotating crystal chopper was operated asynchronously with the storage ring period (Fig. 5.33). In order to be a more useful instrument, the rotating crystal chopper must be able to operate synchronously with the storage ring. We are currently looking into improvements of the rotation devices that will permit synchronous operations.

The other main area of instrumentation development ongoing in the time-resolved program involves a high-speed digital CCD camera. During the last year, the software for operating the camera has been improved significantly. The following aspects have been added or enhanced: user friendliness, ability of executing loop commands within 


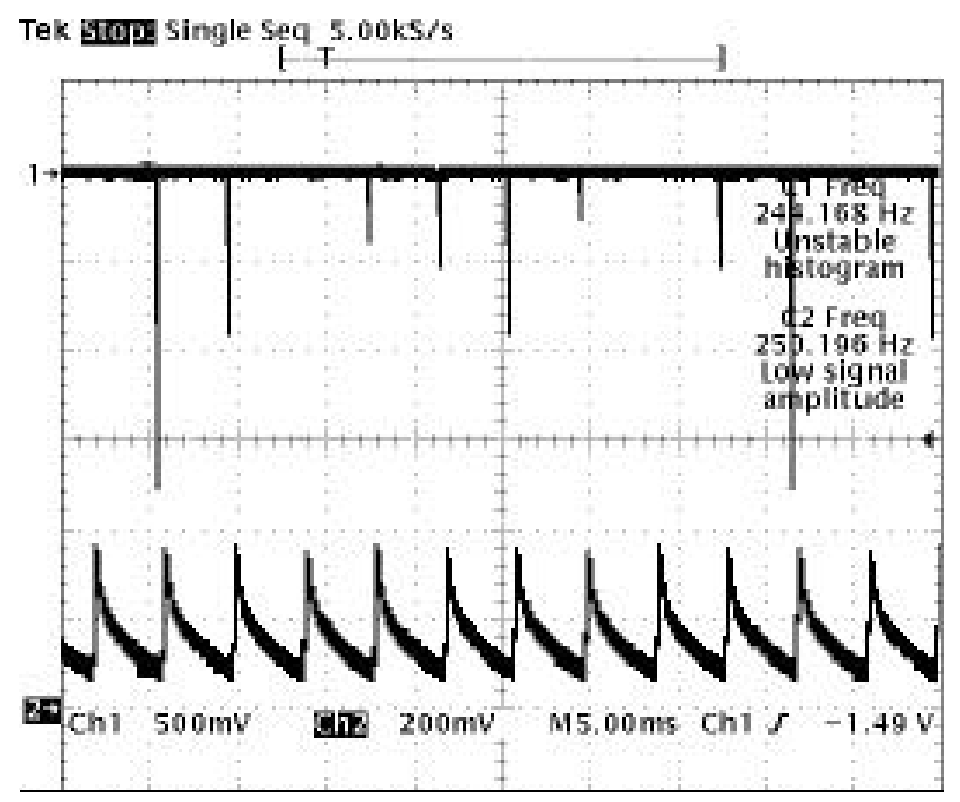

(a)

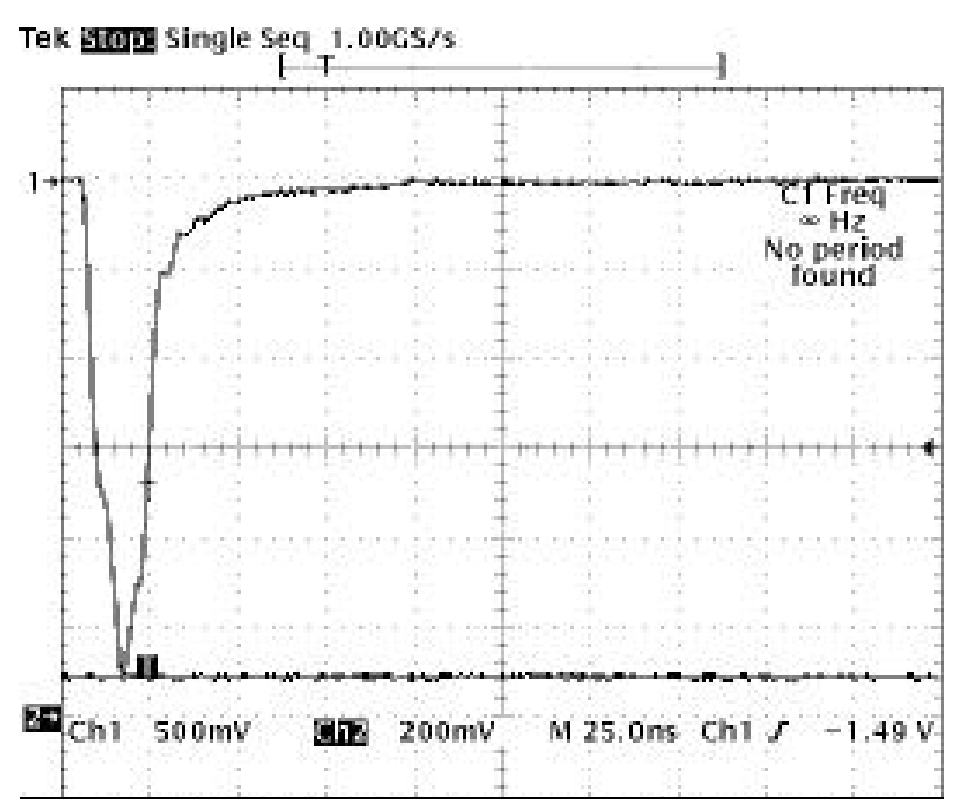

(b)

Fig. 5.33 In (a), the lower trace is from an optical diode, which measures the frequency of the rotating cube, and the upper trace is from an avalanche photodiode (APD) $x$-ray detector. This figure shows that the $x$-rays are now arriving at about $250 \mathrm{~Hz}$. (The crystal is actually rotating at about $63 \mathrm{~Hz}$, but there are four reflecting faces available.) The asynchronous operation of the chopper results in variable $x$-ray pulse amplitudes (and sometimes misses them altogether). Figure (b) shows that each $x$-ray pulse shown in (a) is actually a single bunch (out of typically 25 bunches) from the synchrotron. In this case, the captured bunch is a sextet, which is normally followed by a triplet about 100 ns later. The triplet does not show up because the opening transmission time of the crystal is less than 100 ns at this rotation frequency. 
the script language, synchronization between the camera and other user instruments, such as rapid mixer and stopped-flow devices. Full characterization of the CCD camera, including measurements on time response and $\mathrm{x}$-ray detection efficiency is in progress.

\section{High-Energy X-ray Scattering}

This program focuses on the development of instruments and techniques that utilize $\mathrm{x}$ rays in the energy range from about $20 \mathrm{keV}$ to $200 \mathrm{keV}$. Many aspects of instrumentation (on 1-BM) were described in the XFD Progress Report 1996-97. The unique capabilites of this beamline have attracted many scientists to perform numerous materials science studies during 1997-98. In the following, we present a brief summary of these measurements.

Several experiments were conducted in which high-energy $x$-rays were used to study noncrystalline materials. For these experiments, the bent-crystal monochromator (BCM) in 1-BM-B was used to produce an $\mathrm{X}$-ray beam with an energy of $61 \mathrm{keV}$. The BCM consists of one long crystal of either (111) or (220) oriented silicon that is bent (meridionally) to horizontally focus the beam. The crystal was cut to have an asymmetry that allowed for a monochromatic focus within the 1-BM-B station for energies greater than $50 \mathrm{keV}$. The diffractometer was operated in a vertical scanning geometry to take advantage of the superior angular divergence in that direction. With the use of the BCM, the typical time to complete a scan on an amorphous material sample was around one hour. This configuration is a significant improvement over the setups used for previous experiments on amorphous or liquid materials.

In collaboration with scientists from the University of Missouri-Rolla and MSD at Argonne, we examined a series of depleted uranium oxide glass alloys. Glass alloys such as these are candidates for nuclear waste storage and, hence, knowledge of the structure of such materials is crucial to understanding its long-term stability. The glasses studied were iron phosphate glasses alloyed with various amounts of uranium oxide ranging from $0 \%$ to $24.5 \mathrm{wt}$. $\%$. Figure 5.34 shows the scattering from three of the glass alloys. The effect of $\mathrm{UO}_{2}$ composition upon the scattering is dramatic, as might be expected considering the heavy weighting of uranium in the x-ray scattering. Figure 5.35 shows the total correlation function, $\mathrm{T}(\mathrm{r})$ for data collected with high-energy $\mathrm{x}$-rays and with neutrons. The enhanced contribution to the $\mathrm{x}$-ray $\mathrm{T}(\mathrm{r})$ by uranium is clearly evident. These results are being used in combination with Raman, Mossbauer, and x-ray absorption spectroscopy to help resolve structural issues for these glass alloys (Marasinghe et al., 1998).

Another study was conducted in collaboration scientists from MSD at Argonne and the Osaka Institute of Technology. Glass pellets of $\mathrm{GeO}_{2}$ were post-processed by compression and heating to achieve densities of 4.08 and $4.18 \mathrm{~g} / \mathrm{cm}^{3}$ (normal density for $\mathrm{GeO}_{2}$ is $3.62 \mathrm{~g} / \mathrm{cm}^{3}$ ). The long-range network topology of glasses in general is a subject of interest. Studying the compression-induced change in the scattering from $\mathrm{GeO}_{2}$ was intended to provide insight into this matter, as well as into the rigidity of bond-angle-dependent potentials. Scattering from the two highdensity samples and from a normal density sample is shown in Fig. 5.36, which shows 


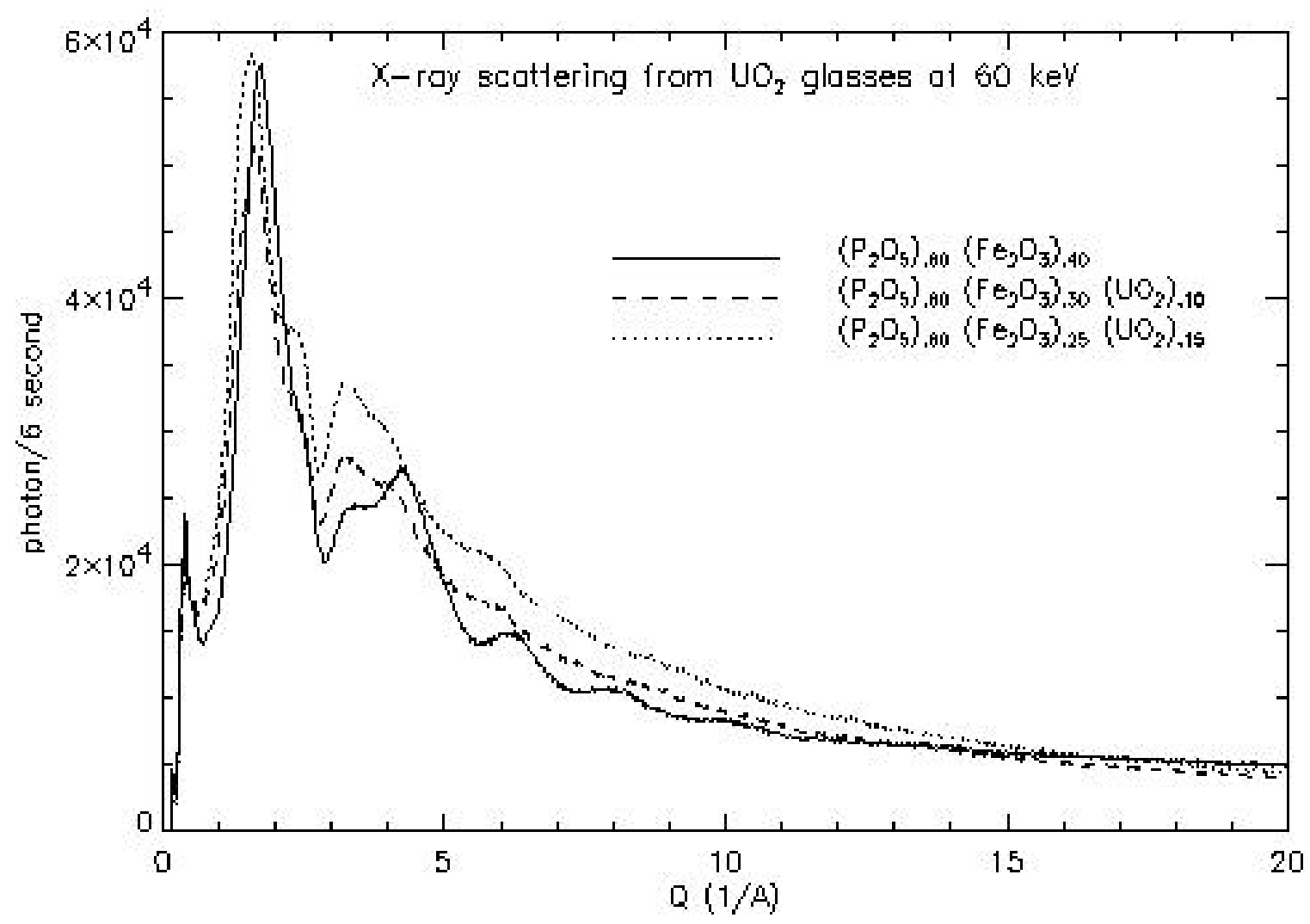

Fig. 5.34 The $x$-ray scattering for three glasses with different $\mathrm{UO}_{2}$ compositions.

the high $Q$ scattering $(Q=4 \pi \operatorname{Sin} \theta / \lambda)$ data for the uncompressed sample. Structure is readily apparent out to at least $20 \AA^{-1}$, indicating the utility of high-energy x-rays to collect scattering out to high $\mathrm{Q}$. The T(r) for these samples is shown in Fig. 5.37. From these results, we see that the first two shells are largely unchanged by the compression, but that significant differences exist for shells past the first two.

With a group of scientists from Indiana University, we studied the structure of alkali tellurite glasses. These glasses have the general formula $\left(\mathrm{Na}_{2} \mathrm{O}\right)_{\mathbf{X}}\left(\mathrm{TeO}_{2}\right)_{1-\mathrm{x}}$, where $\mathrm{TeO}_{2}$ is the glass former and $\mathrm{Na}_{2} \mathrm{O}$ is the modifier. Varying the concentration of the latter controls bulk properties, such as the glass-forming temperature and nonlinear optical response. The strong nonlinear optical response, which arises from a polarizable lone Te electron pair, combined with the good chemical durability and transparency in the visible and near infra-red (IR) ranges, makes these glasses a candidate for photonics applications. From previous results obtained by neutron scattering and from nuclear magnetic resonance experiments, structural models had been developed but without sufficient information to determine which was correct for any given composition. By adding the results from the high-energy $\mathrm{x}$-ray scattering experiments to their other results, the Reverse Monte Carlo algorithm was used to give estimates on compositions for various 


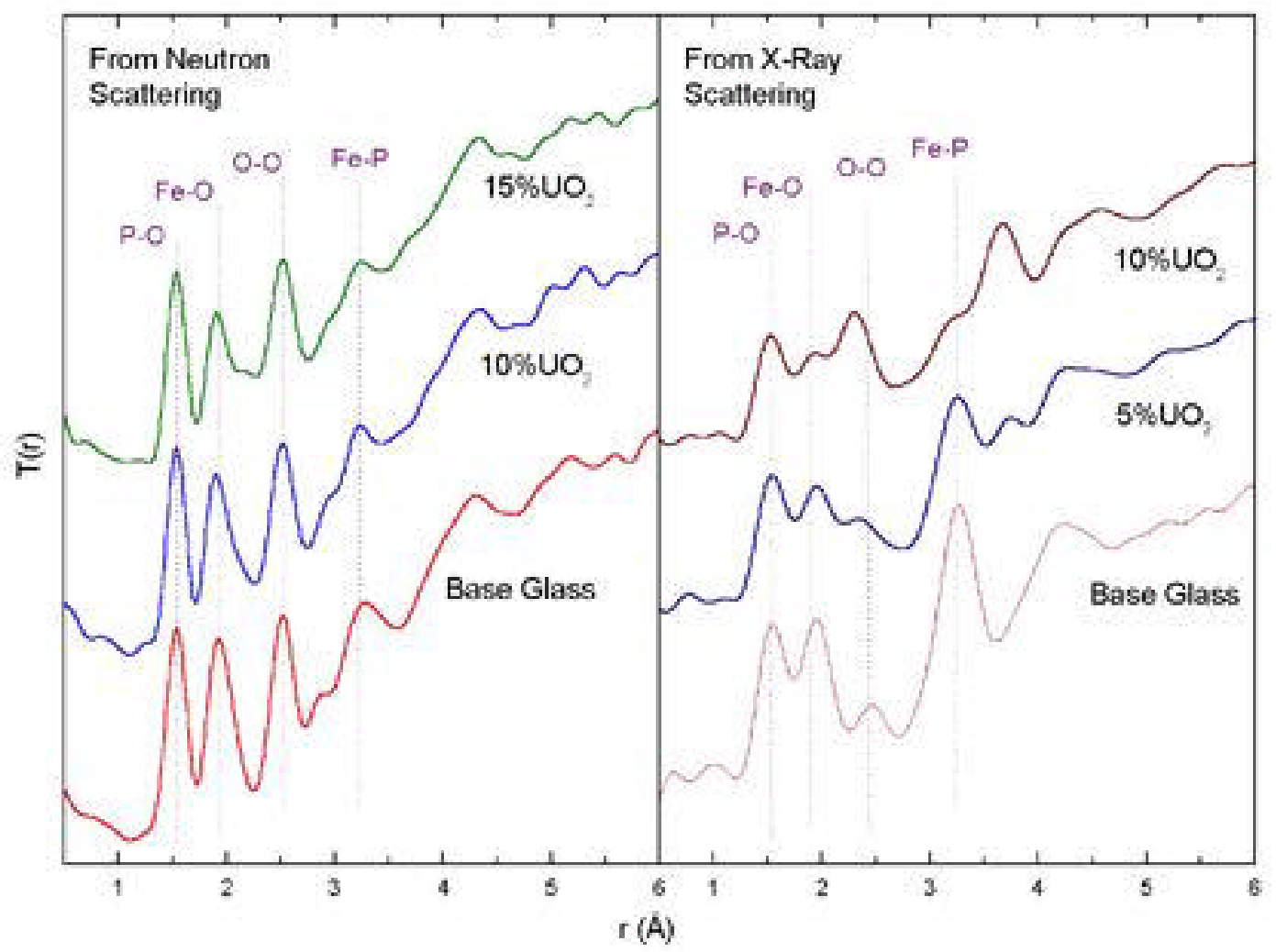

Fig. 5.35 The total correlation function derived from neutron and $x$-ray scattering for three glasses with different $\mathrm{UO}_{2}$ compositions.

structural components (McLaughlin et al., 1998).

The high-energy scattering capability provided at beamline 1-BM is unique, and many scientists have taken advantage of it to study materials science problems requiring $\mathrm{x}$-ray scattering data for full understanding.

\subsubsection{Sector 2}

\section{2-BM Beamline and Optics}

The 2-BM beamline, consisting of a bending magnet source and the 2-BM-A and 2-BM-B experiment stations, has been commissioned and operational since the end of 1997. The beamline as a whole has demonstrated excellent performance. The last major optical component to be installed was the M2 mirror in 2-BM-B, a 1.2-m-long Si flat, side-cooled mirror with $\mathrm{Si}, \mathrm{Rh}$, and $\mathrm{Pt}$ stripes on its reflective surface for broadband energy selection up to $\sim 35 \mathrm{keV}$. This mirror and its cousin in 2-BM-A have performed very well, demonstrating reflectivity curves that are within $5 \%$ of the expected theoretical reflectivities. The last remaining optical component to be installed as per the Sector 2 Final Design Report is a double-multilayer monochromator (DMM) for medium-bandwidth energy selection. 


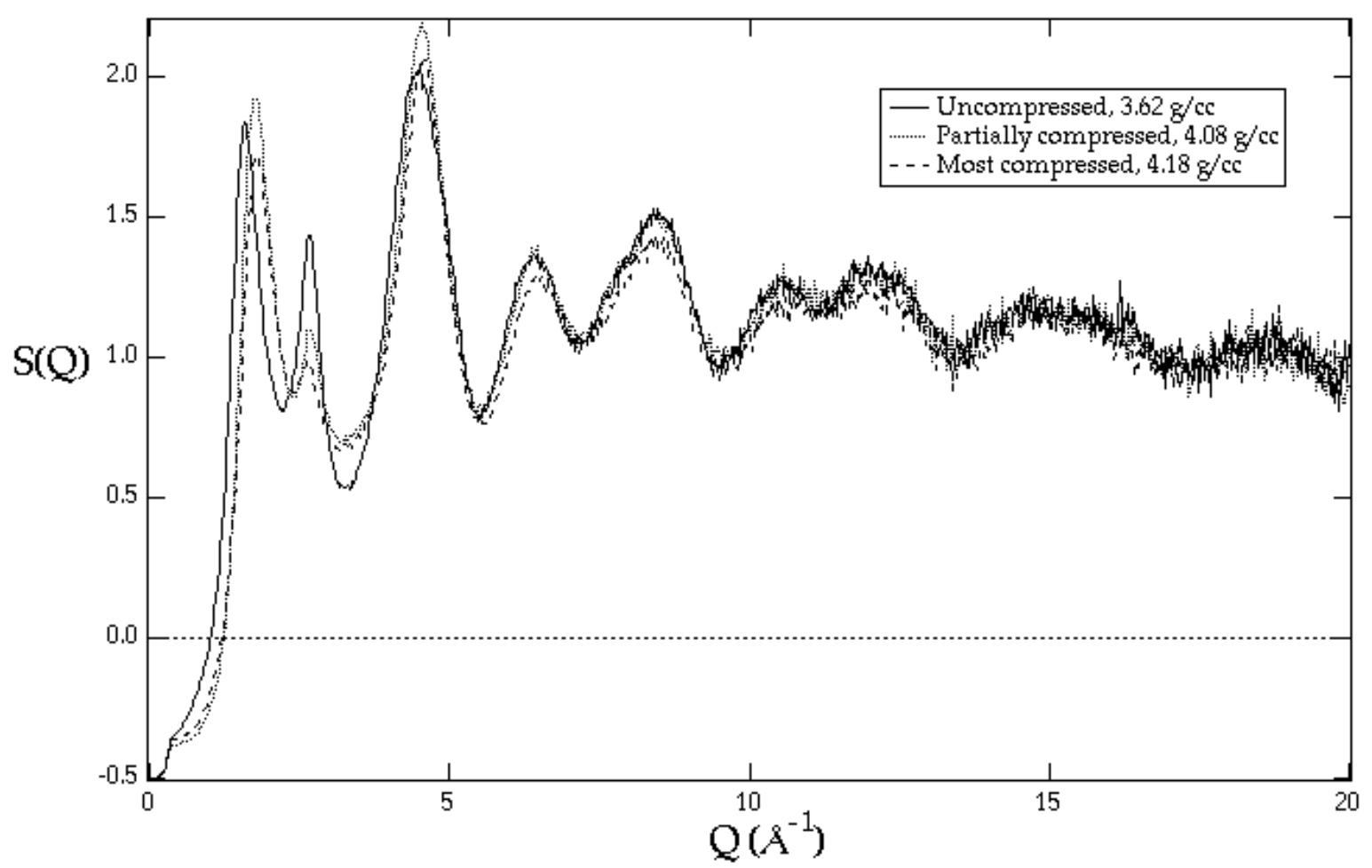

Fig. 5.36 The x-ray structure factor for normal $\mathrm{GeO}_{2}$ glass and for two densified $\mathrm{GeO}_{2}$ glasses.

This instrument is slated to be commissioned in October 1998.

\section{Optics Characterization and Advanced Technique Development}

Significant progress has been made at 2-BM-B on testing and characterization of zone plate lenses for high-resolution focusing of x-rays and on development of replicable x-ray multilayer optics. For microfocusing applications with high-energy $\mathrm{x}$-rays, zone plates can be stacked together to increase their efficiency. By precisely aligning two zone plates optimized for $8 \mathrm{keV} \mathrm{x}$-rays, we showed that they can constructively interfere with one another and produce a combined efficiency of $>30 \%$ for
$16 \mathrm{keV} \mathrm{x}$-rays, which is close to the optimal value that can be achieved for a zone plate with rectangular profile. At 2-BM-B, we have also made measurements of the reflectivity and scattering from multilayers on Ni-electroform substrates for $\mathrm{x}$-ray astronomy applications. These data were compared with AFM and WYCO roughness measurement to assess their validity at different spatial frequencies. The results showed that precise replicas of a master mirror surface can be made repeatedly by electroforming followed by liftoff from the master.

On the advanced technique development front, we have assembled and tested instrumentation for microtomography experiments that uses a high-spatialresolution CCD camera. Either absorption or 


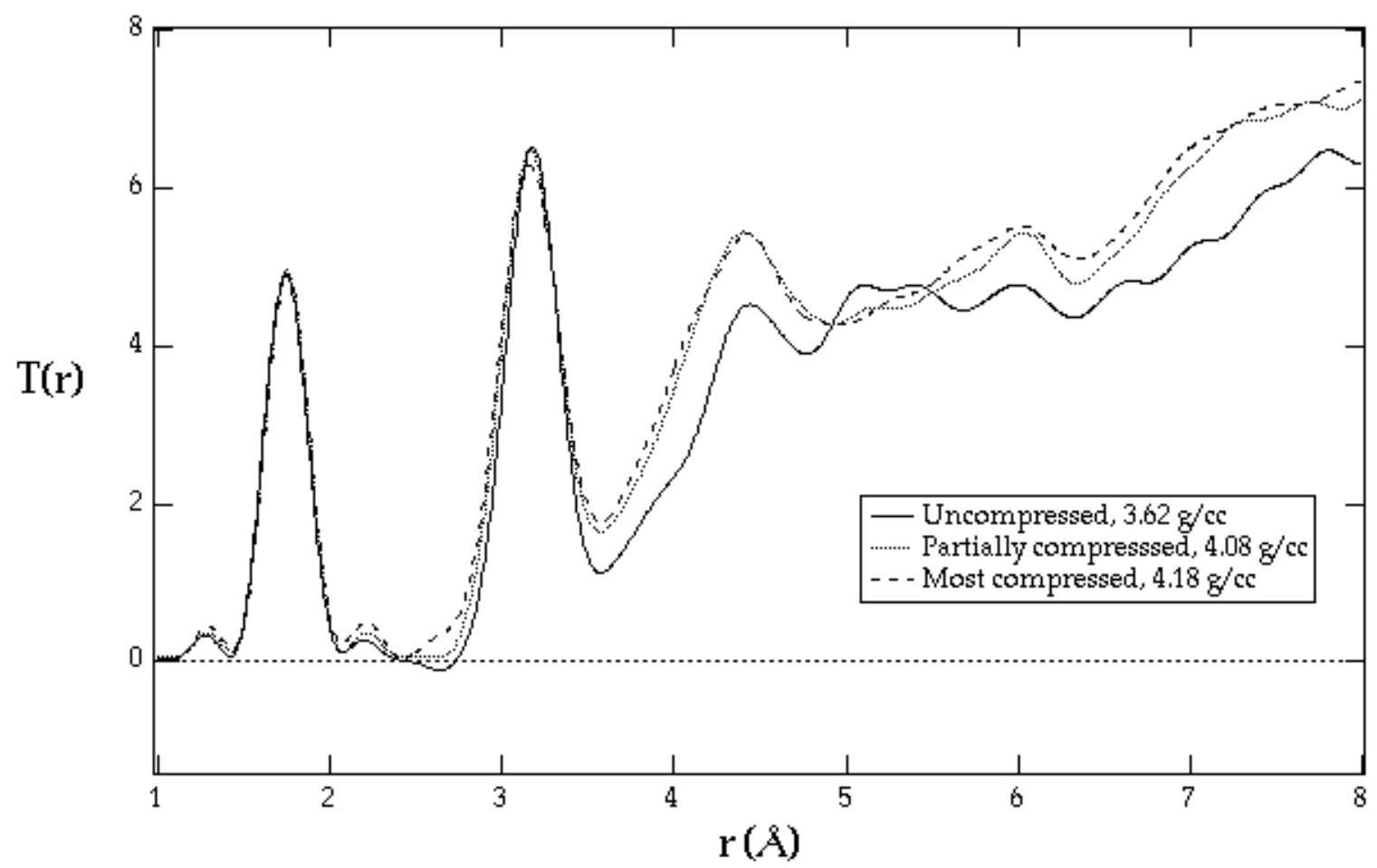

Fig. 5.37 The total correlation function for normal $\mathrm{GeO}_{2}$ glass and for two densified $\mathrm{GeO}_{2}$ glasses.

phase contrast images of the sample can be obtained with this instrumentation. Fast data collection ( $\sim 1$ projection/s) is achieved with a sample rotation angle range of $180^{\circ}$. Using the filtered back-projection algorithm to reconstruct the tomographic data, we demonstrated a resolution of $1-2 \mu \mathrm{m}$ in all three spatial dimensions. In collaboration with the ANL Environmental Research Division (ER), properties of soil aggregates, such as density distribution, porosity, and connectivity of pores, were studied using the microtomography setup (Fig. 5.38). The structure of soil samples ( 1 $\mathrm{mm}$ in size) from different field locations and the mechanism of soil expansion and contraction by hydrated ions were examined. $\mathrm{X}$-ray tomography provided repeated nondestructive evaluations of the sample throughout the wetting and drying cycles.

\section{Deep X-ray Lithography}

The deep x-ray lithography (DXRL) program at 2-BM has been highly successful in the last year. Among the more notable achievements, we developed new mask technology that is especially suitable for DXRL. The mask technology uses 1-mm beryllium wafers as substrates, with either silicon nitride or boron coatings to act as adhesion and protection layers. We have completed finite element analysis modeling that shows the 1-mm beryllium wafers to be most suitable for both thermal management 


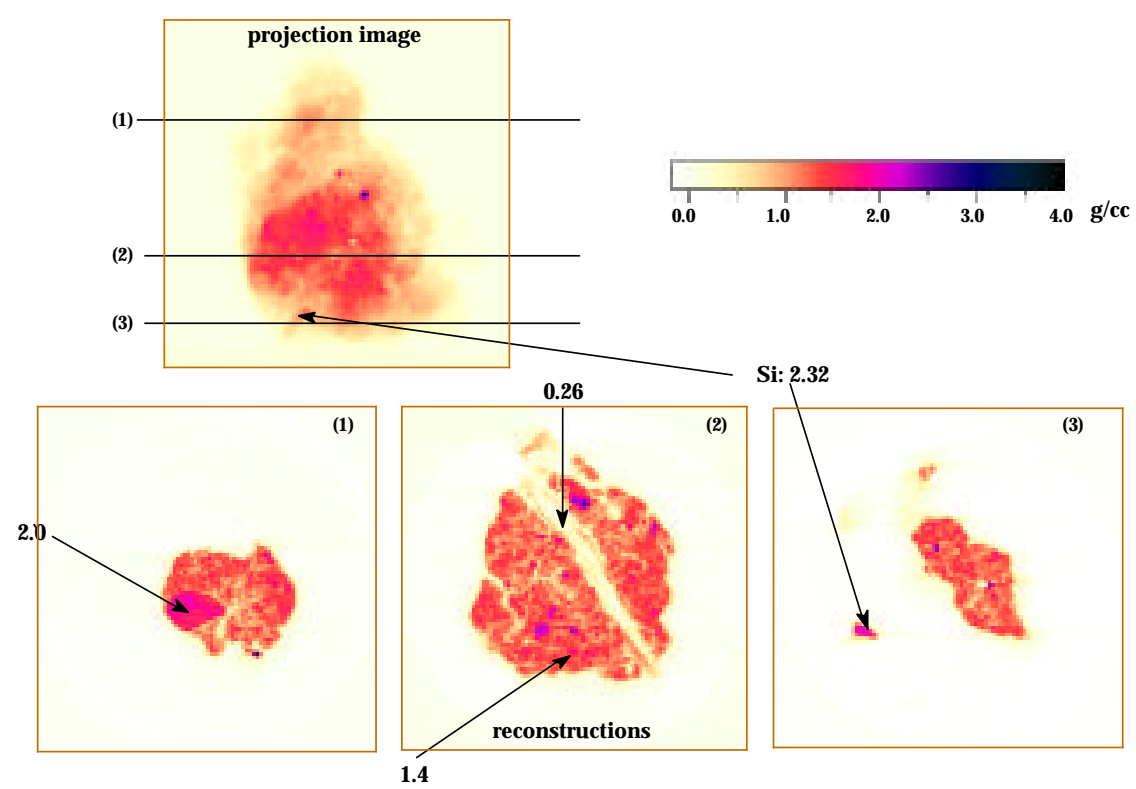

Fig. 5.38 Density map of a soil aggregate from an uncultivated field. The top figure is an absorption image of the sample as collected by the CCD camera. The bottom three images are reconstructed slices through the sample. In addition to the pores, a root canal with organic debris, a sandstone, and several heavy particulates are visible.

and dimensional stability, with graphite substrates as a potential lower cost alternative. Absorber patterns in 30-80 $\mu \mathrm{m}$ thick gold have been electroformed onto the beryllium after soft x-ray pattern transfer into $100 \mu \mathrm{m}$ plus thick spun PMMA from a $2 \mu \mathrm{m}$ conformal gold mask prepared by optical lithography. In collaboration with LaTech-IfM, we have tested prototype masks with graphite substrates and a $30 \mu \mathrm{m}$ gold absorber. Preliminary results have encouraged us to incorporate graphite substrates into our mask technology.

We have also established gold electroforming capabilities as part of this program. These electroforming baths have been used in preparing some of the prototype masks described above, and we have further improved the gold electroforming by working with Dover Industrial Chrome and vendors of the plating baths, resulting in different optimized baths for various applications. An example of this is shown in Fig. 5.39. We continue to work with Dover for the electroforming of nickel and copper structures and have commenced transferring the copper electroforming technology to the APS.

Employing the same gold-on-beryllium technology developed for mask fabrication, we have successfully fabricated for NASA a prototype $\mathrm{x}$-ray coded aperture array. NASA tested the prototype for potential application in a gamma-ray telescope satellite, earning 


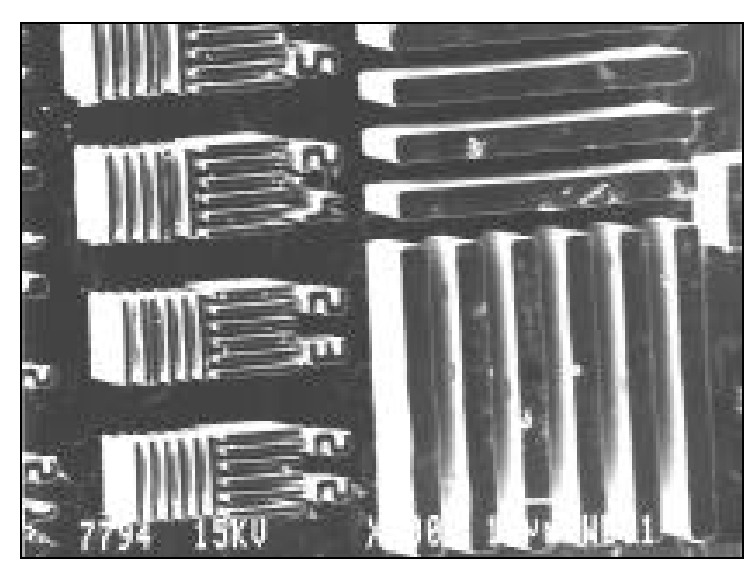

Fig. 5.39 Hard x-ray mask fabricated by XFD for DXRL with resolution test pattern showing features down to $\sim 2$ microns wide in 25-micron-tall gold on a 300-micronthick silicon substate.

an achievement award for our collaborator. Working with the RF group in ASD, we have fabricated prototype mm-wave $R F$ accelerator structures suitable for RF testing. We have begun the fabrication of precision pinholes and slits for use at the APS. We have initiated collaborations with various groups in MSD at ANL in the area of material applications for microelectromechanical systems (MEMS), as well as external collaborations with University of Chicago, University of Illinois at Chicago, Forschung Zentrum Karlsruhe (Germany), and KETI (South Korea).

We have completed a series of test exposures and development of 1-mm PMMA sheets under varying exposure conditions (mirror angles, scan speeds, dose rates) at 2-BM. These exposures have been used to determine the optimal exposure and development conditions, how to limit damage to PMMA during exposure, and methods for improved adhesion. As a result of these studies, we have optimized our standard processes to improve the achievable resolution and depth attained using DXRL. Examples of this are shown in Fig. 5.40 (a) and (b). By successfully producing 1-6 mm thick PMMA structures on various substrates, we have also been able to demonstrate the advantages of using an APS bending magnet source for DXRL. These structures include $100 \mu \mathrm{m}$ towers and holes and resolution test patterns for tests of development and adhesion. Aspect ratios of 100:1 have been achieved in a 2.5-mm-thick resist.

Finally, we have installed and commissioned a new precision $\mathrm{x}$-ray scanner in the 2-BMB station, and preliminary testing shows its capability to meet specifications. The scanner will be further enhanced by the addition of a rotational stage that will allow production of conicals and compound geometries.

\section{2-ID-B Beamline and Optics}

Most of the key optics and components of the 2-ID-B beamline were installed and commissioned by the end of 1997 . Of major importance to the 2-ID-B scientific program is the successful commissioning and characterization of the $5.5-\mathrm{cm}$-period soft X-ray undulator, known as U5.5. To date we have operated it and commissioned the entirety of 2-ID (all three branch lines) at a minimum magnetic gap of $14.0 \mathrm{~mm}$, corresponding to a fundamental energy of $635 \mathrm{eV}$ and a maximum total power output of $7 \mathrm{~kW}$. We have found that the 1.2-m-long M1 mirror ( $\mathrm{Si}$ substrate, $\mathrm{Si}, \mathrm{Rh}$, and $\mathrm{Pt}$ stripes operating at $0.15^{\circ}$ of incidence) in the 2-ID-A first optics enclosure performs exceptionally stably and reliably under the extreme power load of this source, the "hottest" undulator at the APS. Although we have yet to perform a precision absolute flux 
(a)

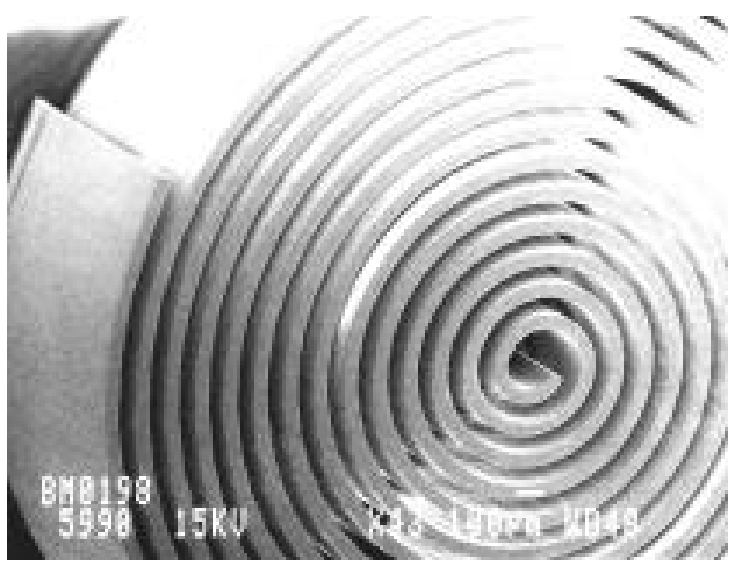

(b)

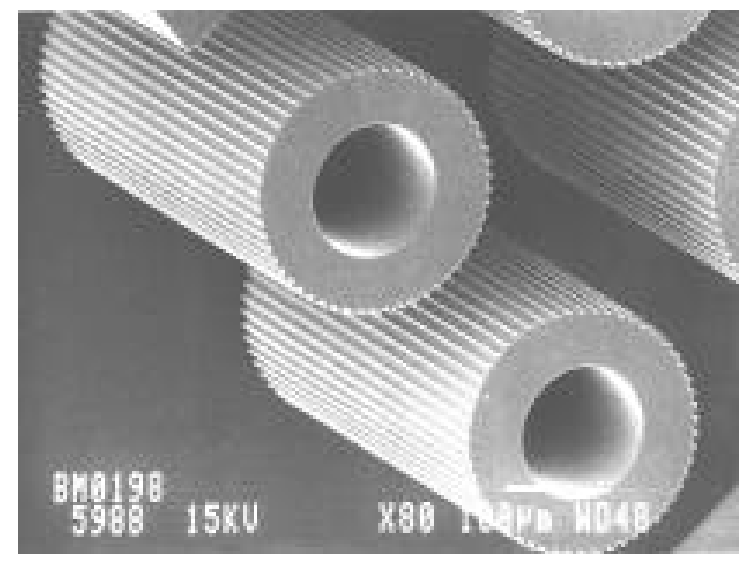

Fig. 5.40 1-mm-tall structures in PMMA reproduced using the hard $x$-rays from the APS and a gold-on-graphite $x$-ray mask fabricated as part of a collaboration between XFD, the Institute for Micromanufacturing at Louisiana Tech University (Ruston, LA), and Forschung Zentrum Karlsruhe (Germany).

measurement, the measured flux at the beamline end station is within an order of magnitude of that predicted using an Al vacuum photodiode. Moreover, by measuring the flux in the 120-nm diffraction-limited microfocus of a Fresnel zone plate with an avalanche photodiode, we have established that the coherent flux incident on the zone plate, and thus the source brilliance, is in the expected range.

The three soft $\mathrm{x}$-ray pink beam position monitors (BPMs) were also installed and tested. By introducing small angular displacements in the $\mathrm{x}$-ray beam with the M3B mirror, we determined that the spatial resolution of the BPMs is $1-2 \mu \mathrm{m}$, which is sufficient precision for closed-loop beam steering in combination with the M2B or M3B mirrors and for beam position monitoring. We have also completed much of the characterization and calibration of the 2-ID-B spherical grating monochromator (SGM), including measurement of a resolving power $(\mathrm{E} / \Delta \mathrm{E})>3000$ at the $\mathrm{Si} 1 \mathrm{~s}$ absorption edge using the $1800 \mathrm{gr} / \mathrm{mm} \mathrm{Pt}$ coated grating. The last optical elements to be installed in the 2-ID-B beamline, slated for late 1998, will be multilayer gratings, which are needed to access energies above $\sim 2 \mathrm{keV}$.

\section{High-Resolution Scanning X-ray Microscopy}

Substantial progress has been made on the development of submicrometer scanning microscopy, microtomography, and microspectroscopy in the $1-2 \mathrm{keV}$ energy region at 2-ID-B. The scanning x-ray microscope (SXM) uses a gold zone plate with a 100 -nm-wide outermost zone to form an x-ray microfocus through which the sample is scanned. The measured efficiency of the zone plate at $1.57 \mathrm{keV}$ is about $18 \%$, within $2 \%$ of the theoretical value. Knifeedge scans through the zone plate focus 
indicate the spatial resolution of the instrument is better than $150 \mathrm{~nm}$ (Fig. 5.41). The sample stage consists of a fast, two-axis electrostrictive actuator-driven fine stage with 6-nm resolution atop a stepper-driven coarse stage, and a precision rotational stage for orienting the sample at various angles of incidence to the zone plate focus. We characterized the performance of the SXM using pinhole and grid test samples. The fine stage motion is highly reproducible although
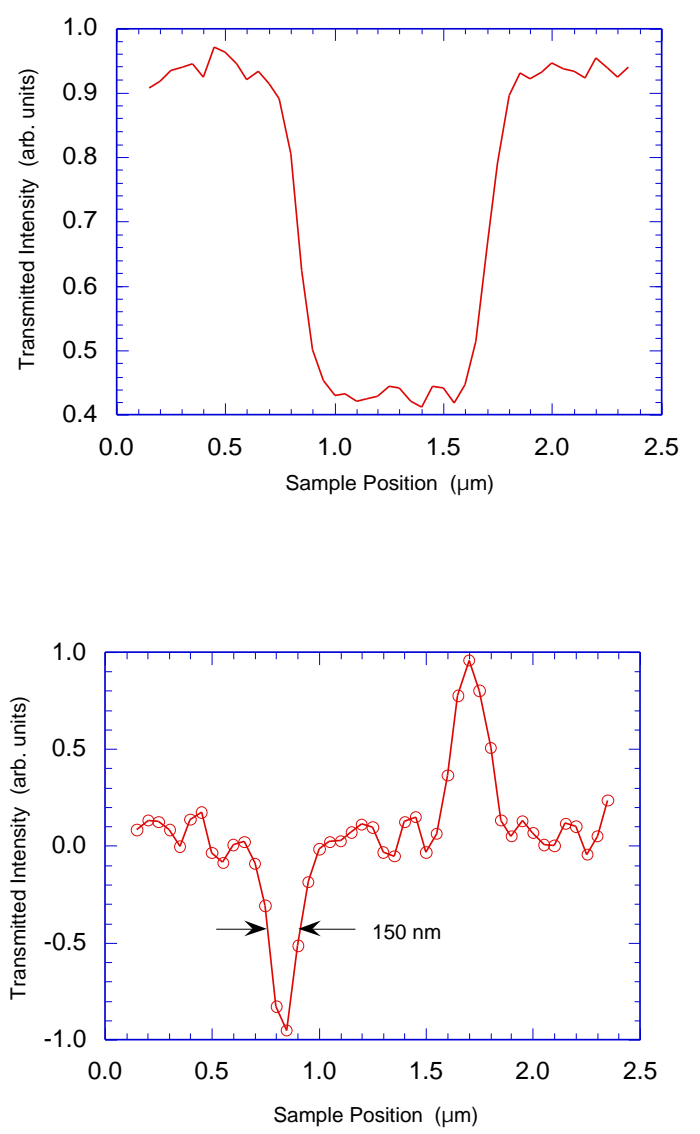

Fig. 5.41 (top) Knife-edge scan across an integrated circuit interconnect, and (bottom) its derivative showing the imaging resolution (focal spot size convolved with 50-nm scan steps and edge of the 500-nmwide interconnect) is about $150 \mathrm{~nm}$. the scan field is somewhat warped; this is currently under investigation. The SXM currently operates in transmission mode using an avalanche photodiode (APD) as the transmitted flux detector. The plan is to deploy a low-energy Ge dispersive detector as a fluorescence detector in late 1998.

\section{Scanning Microtomography and Microspectroscopy of Chips}

As manufacturers of integrated microelectronic circuits (chips) seek to achieve higher component counts and densities, the feature sizes in these devices become correspondingly smaller. This and the higher current densities that may prevail pose additional constraints on the ability of such devices to tolerate electromigration effects as they age. Electromigration is the primary cause of unwanted transport of the materials used to carry electrical current in chips, leading to lattice strains, highresistance conduction paths, and eventual failure. Consequently, a better understanding of electromigration is of tremendous importance to the microelectronics industry. Current means of imaging microelectronic devices, by visible light or by electron microscopy, are mostly limited to the sample surface, necessitating successive chemical or mechanical milling in order to investigate buried layers. Visible photons cannot resolve better than $\sim 0.25 \mu \mathrm{m}$, electrons lead to sample charging, and neither probe is very sensitive to the sample constituents. X-rays in the few-keV energy range can penetrate the many layers in typical devices and uniquely offer elemental and chemical-specific contrast of the materials used to fabricate them ( $\mathrm{Al}, \mathrm{Si}, \mathrm{Ti}$, $\mathrm{Cu}, \mathrm{W}$, etc.). High-resolution 3-D x-ray microscopy therefore offers a better means to image the imbedded structures and buried 
interfaces in chips without damaging them by thin sectioning. Moreover, the capability to perform element and chemical-state specific imaging at sub-micrometer resolution is highly attractive as a way to elucidate the detailed mechanisms behind electromigration processes in chips.

In collaboration with scientists from NIST, Rensselaer Polytechnic Institute, and Intel Corp., we are using the 2-ID-B SXM to image microelectronics devices in 3-D at $100-\mathrm{nm}$ and smaller length scales in an effort to clarify the failure mechanisms due to electromigration effects. The samples we have explored to date, which were fabricated and backside-thinned to $\sim 10 \mu \mathrm{m}$ by Digital Equipment Corp. (DEC), consist of arrays of multilevel junctions of aluminum "interconnects" and tungsten "vias" embedded in an $\mathrm{SiO}_{2}$ matrix. We used the SXM to record a series of high-resolution 2-D scanned projections through the samples at $6.92^{\circ}$ angular increments over a $140^{\circ}$ range of incidence angles. Each projection was $15 \mu \mathrm{m} \times 15 \mu \mathrm{m}$ in area and required about $30 \mathrm{~min}$ to acquire the $300 \times$ 300 pixel scans with a dwell time of 10 ms/pixel. The projections taken at incidence angles above $60^{\circ}$ from normal clearly show the 3-D nature of the sample features, especially the vias, which extend into the depth of the $\mathrm{SiO}_{2}$ matrix. Tomographic reconstructions of the projection data set are underway, from which full 3-D views of the sample can be rendered. This technique, when fully developed, is promising for highresolution 3-D chip inspection and defect analysis.

We also recorded normal incidence projections of the DEC samples over a range of incident photon energies. Figure 5.42 shows two scanned images of a pair of interconnect junctions, taken at two photon energies on either side of the $\mathrm{Al} 1 \mathrm{~s}$ absorption edge (Levine et al., 1998). These data demonstrate the capability of the SXM in combination with the SGM to obtain element-specific images at the 100-nm level.

\section{Development of Soft X-ray XIFS}

The other major experimental effort in the past year at 2-ID-B has focused on development of the tools and techniques necessary to mount and conduct soft x-ray speckle and intensity fluctuation spectroscopy (XIFS) experiments, in collaboration with scientists from ANL MSD and Massachusetts Institute of Technology (MIT). XIFS shows promise for investigation of fluctuations in disordered samples near phase transitions, both at and far from equilibrium. In particular, 1-4 keV $\mathrm{X}$-rays are suited to probing materials composed of the low-Z elements, such as biological objects and polymers. In this work so far we have prepared a highly coherent x-ray beam and used it to record Fraunhofer diffraction patterns with monochromatic and with pink beam (Fig. 5.43), and static speckle patterns produced by a silica aerogel sample. We have used both a 10- $\mu$ m-diameter pinhole and a zone plate to define a $\sim 10-\mu \mathrm{m}$ diameter and a 250 -nm-diameter coherent illumination spot on the sample, respectively. The sample itself was mounted on a 5 - $\mu \mathrm{m}$ - or 15 - $\mu \mathrm{m}$-diameter pinhole, respectively, to block parasitic scattering from previous apertures in the beam. The speckle patterns were recorded initially by a scanning pinhole and the APD; we now use a CCD camera in direct-detection mode for its greater speed and simplicity of use. With the CCD camera, we have learned that use of a zone plate to increase the flux density 
(a)

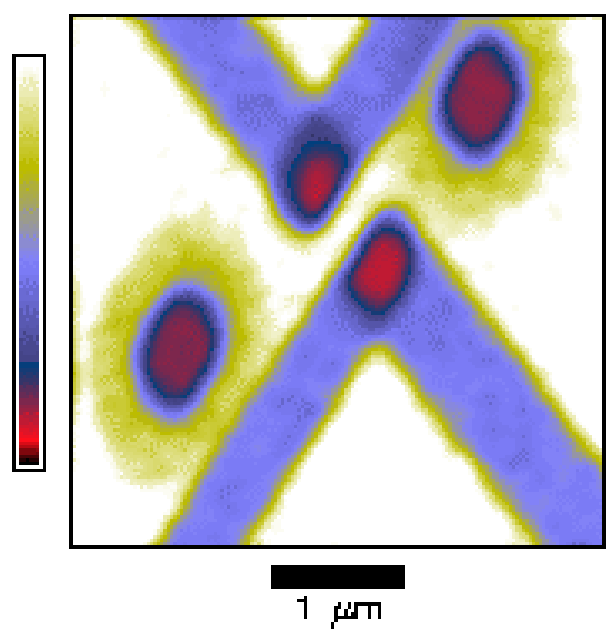

(b)

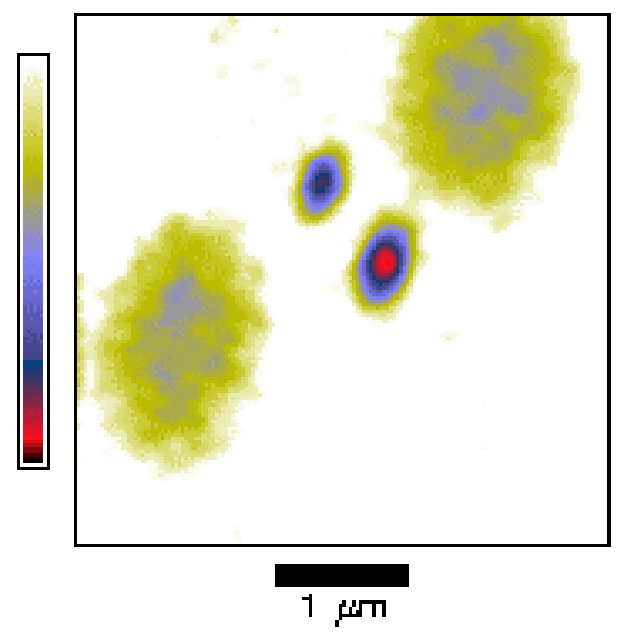

Fig. 5.42 Images of two Al/W integrated circuit interconnect junctions taken at (a) 1563 $\mathrm{eV}$ and (b) $1553 \mathrm{eV}$. The aluminum interconnects forming the junctions become nearly transparent on the low-energy side of the Al 1s absorption edge (1559 eV), whereas the small tungsten via joining the interconnects still appears dense. White and red indicate high and low transmission, respectively.

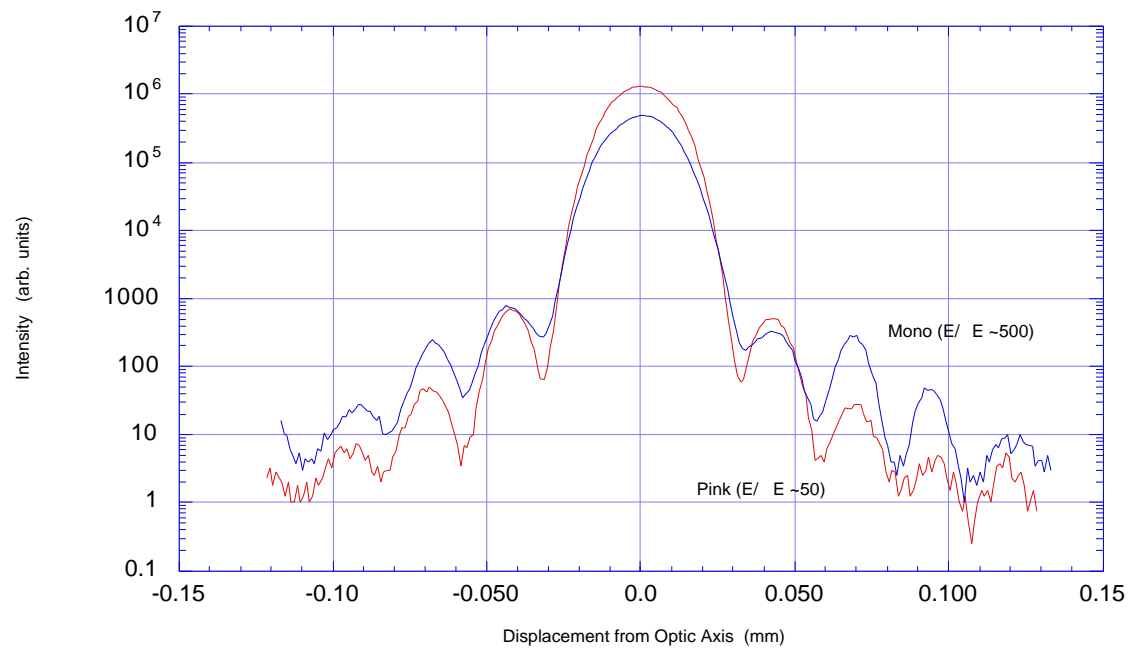

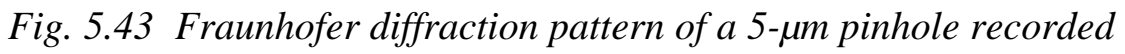
with coherent 1793-eV $x$-rays, using both monochromatic and pink beam at a distance of $217 \mathrm{~mm}$. 
on the sample and to enlarge and simplify the speckle pattern is advantageous. It also allows for a shorter working distance so that speckles at higher momentum transfer can be observed. Furthermore, the speckle size can be matched to the detector geometry by adjusting the position of the sample with respect to the microfocal plane. Lastly, we showed that the speckle contrast increases for energies just above the absorption edge for major elemental constituents in the sample, in this case, above the Si 1s edge in the aerogel. Figures 5.44 (a) and (b) show speckle patterns recorded with the CCD camera in the pinhole and zone plate setups, respectively.

\section{2-ID-C Beamline and Optics}

The soft x-ray polarization and spectroscopy (XPS) beamline, 2-ID-C, is the last branch line of 2-ID to be constructed. As a result the 2-ID-C beamline is still under commissioning and final alignment status. However, the past year has seen great progress towards declaring the 2-ID-C beamline operational. All major components as far as the first diagnostic end station have been tested, integrated into the EPICS control system, and subjected to initial measurements and characterization. In particular, the $\mathrm{M} 2 \mathrm{C}$ horizontal focusing mirror, $\mathrm{M} 3 \mathrm{C}$ vertical focusing mirror, highheat-load entrance slit, exit slit, and two of the gratings (600 grooves $/ \mathrm{mm}$ and 1200 grooves $/ \mathrm{mm}$ ) for the 2 -ID-C beamline have been commissioned and aligned down to the current minimum allowable gap $(14.0 \mathrm{~mm})$ of the U5.5 undulator.

We have made preliminary $\mathrm{x}$-ray absorption measurements utilizing the diagnostic end station. Figure 5.45 shows the $\mathrm{x}$-ray absorption spectrum at the $\mathrm{Gd} 3 \mathrm{~d} 5 / 2$ absorption edge of gadolinium oxysulphide. The measured resolving power of $\sim 6000$ approaches the predicted theoretical (a)

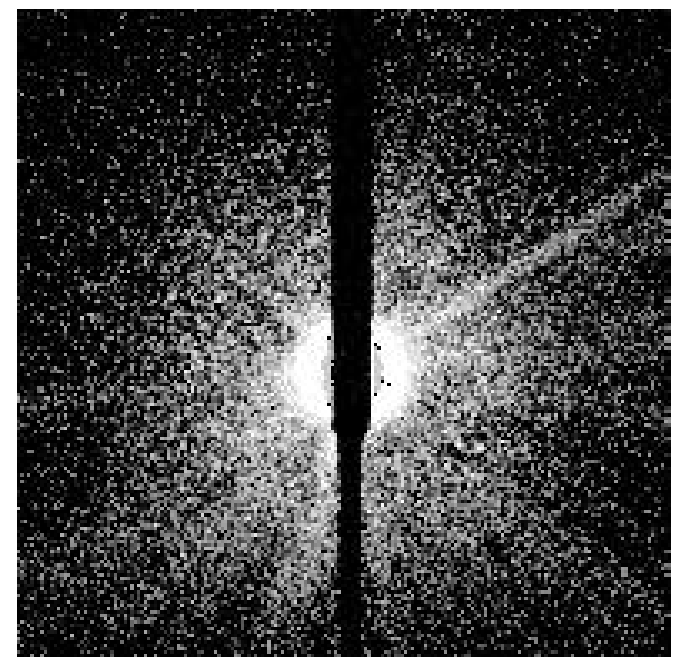

(b)

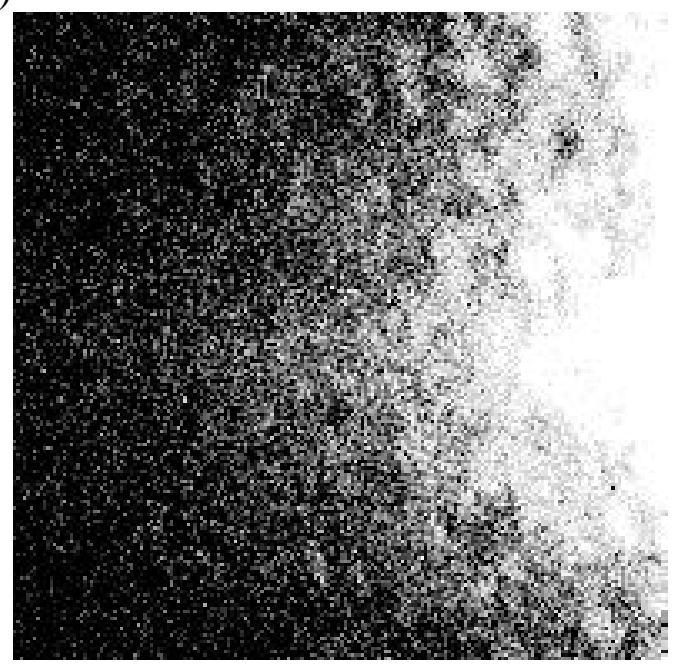

Fig. 5.44 High contrast speckle patterns recorded with (a) a pinhole setup and (b) a zone plate setup. 


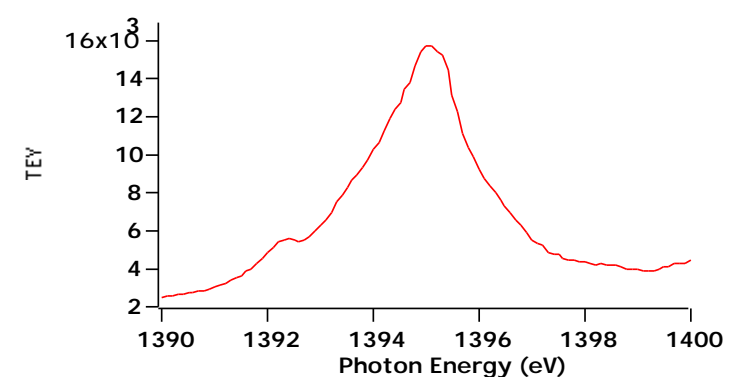

Fig. $5.45 \quad X$-ray total electron yield (TEY) spectrum of gadolinium oxysulphide recorded at the $G d 3 d 5 / 2$ absorption edge

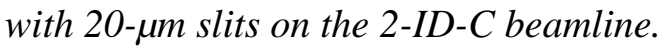

performance of the SGM. Estimation of the resolving power utilizing $\mathrm{x}$-ray absorption data is difficult at these resolving powers as the resolution approaches the natural line width. Future development of the XPS end station will allow for more accurate resolving power determination by looking at narrow core lines and Fermi levels.

The development of the XPS end station and a x-ray fluorescence end station will continue in parallel with the final commissioning and alignment of the beamline. The plans call for development of productive programs in $\mathrm{x}$-ray absorption, $\mathrm{x}$-ray emission, and $\mathrm{x}$-ray photoelectron spectroscopies in the $0.5-3.0 \mathrm{keV}$ energy range, with a particular emphasis upon the lanthanide series.

\section{2-ID-D/E Beamline and Optics}

The 2-ID-D/E branch line was also completed by the end of 1997 . Most of our construction efforts since then have been devoted to development of the 2-ID-E and 2-ID-D end stations, commissioning of new detectors and scan systems, and refinement of the experimental apparatus. In the area of microfocusing optics, the performance of the Fresnel zone plate was significantly enhanced in three areas: (1) higher spatial resolution, (2) higher focusing efficiency, and (3) extension to higher energies.

Our principal achievement was to demonstrate for the first time in the hard $\mathrm{x}$-ray spectral region that Fresnel zone plates are capable of focusing a x-ray beam to a submicrometer spot size with a flux density gain at the focus of more than four orders of magnitude. We obtained a full-width-halfmaximum (FWHM) focal spot size of $150 \mathrm{~nm}$ at the first-order focus of a zone plate with a 100-nm outermost zone width (Fig. 5.46). For a partially coherent beam (no source aperturing), this represents a very high gain of $3 \times 10^{4}$ in the $x$-ray flux density at the focus compared to the incident beam. If the beam is fully coherent, by aperturing the source size, the gain can be further increased to a record $1.2 \times 10^{5}$. This is probably the highest flux density gain reported for any microfocusing x-ray optic, and high spatial resolution with high gain is essential for many applications of $\mathrm{x}$-ray microprobes. For instance, our recent measurements on imaging trace elements in bacteria were made possible only with the availability of these high-resolution optics. On the same zone plate, we also demonstrated a FWHM focal spot size less than $90 \mathrm{~nm}$ using the third-order focus.

Another important performance criterion of microfocusing optics is their efficiency. We have made important progress in improving the diffraction efficiency of $\mathrm{x}$-ray zone plates by using a blazed zone profile. Although an ideal phase zone plate with rectangular zone profile has a first-order diffraction efficiency of $40 \%$, a significant fraction (at least 60\%) of the incident 


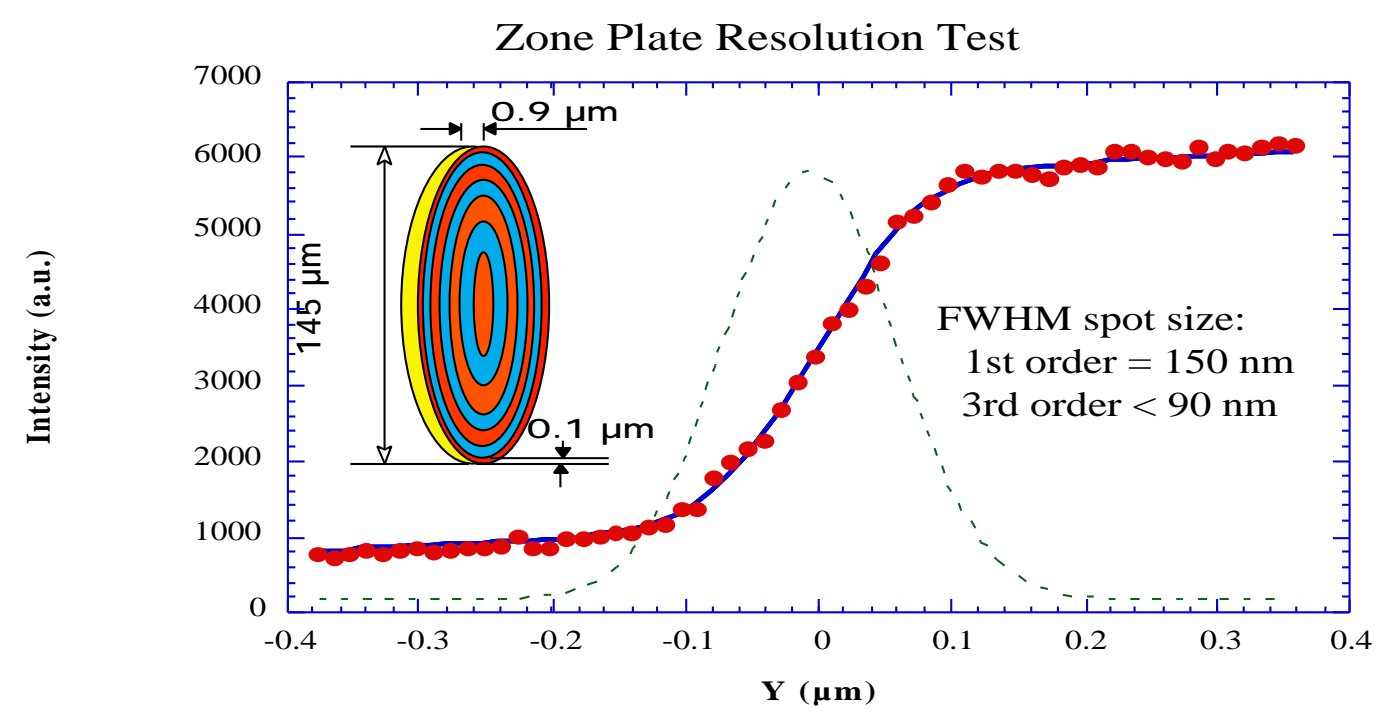

Fig. 5.46 Fluorescence intensity (red dots) from a Cr knife-edge as it was scanned through the focus of a high-resolution zone plate. An error function (green dotted line) with $150 \mathrm{~nm}$ FWHM was integrated and fitted to the fluorescence data (blue solid line).

photons are delivered into the other diffraction orders. Instead, by blazing the zone profile (approximated in this case by a staircase with as little as four steps), we demonstrated focusing efficiencies of $39 \%$ and $45 \%$ with a circular and a linear Fresnel zone plate (Fig. 5.47), respectively, surpassing the $40 \%$ limit of a conventional phase zone plate with rectangular zone profile. More importantly, as more x-rays are delivered to the first-order focus, fewer photons end up in the unwanted orders. The result is a much higher peak-to-background ratio. On the zone plate with stepped profile, the measured flux density of the focused spot is $3.6 \times 10^{3}$ times higher than the background. Not only is this beneficial for imaging and coherence applications, it also allows the implementation of large sample environmental chambers by removing the need of an order-sorting aperture close to the sample.
Most of the zone plates developed so far were optimized for the $8-20 \mathrm{keV}$ energy range. Although there is great interest in using zone plates for high-energy applications (20-100 keV), the fabrication process becomes increasingly challenging as larger thicknesses are required to maintain a $\pi$ phase shift at higher energies. An alternative is to stack multiple zone plates, with a common optical axis, for high-energy use. This not only increases the capabilities of currently available zone plates by several fold, it also opens up a new realm of microfocusing applications in the highenergy regime. In an experiment performed at 1-ID-C (which has high x-ray energy capabilities), we showed that stacking and aligning two zone plates with an individual efficiency of $13 \%$ when used separately yields a combined efficiency of $25 \%$ at $40 \mathrm{keV}$, which is as good as the efficiency we normally obtained for most zone plates at lower energies (8-20 keV). More 

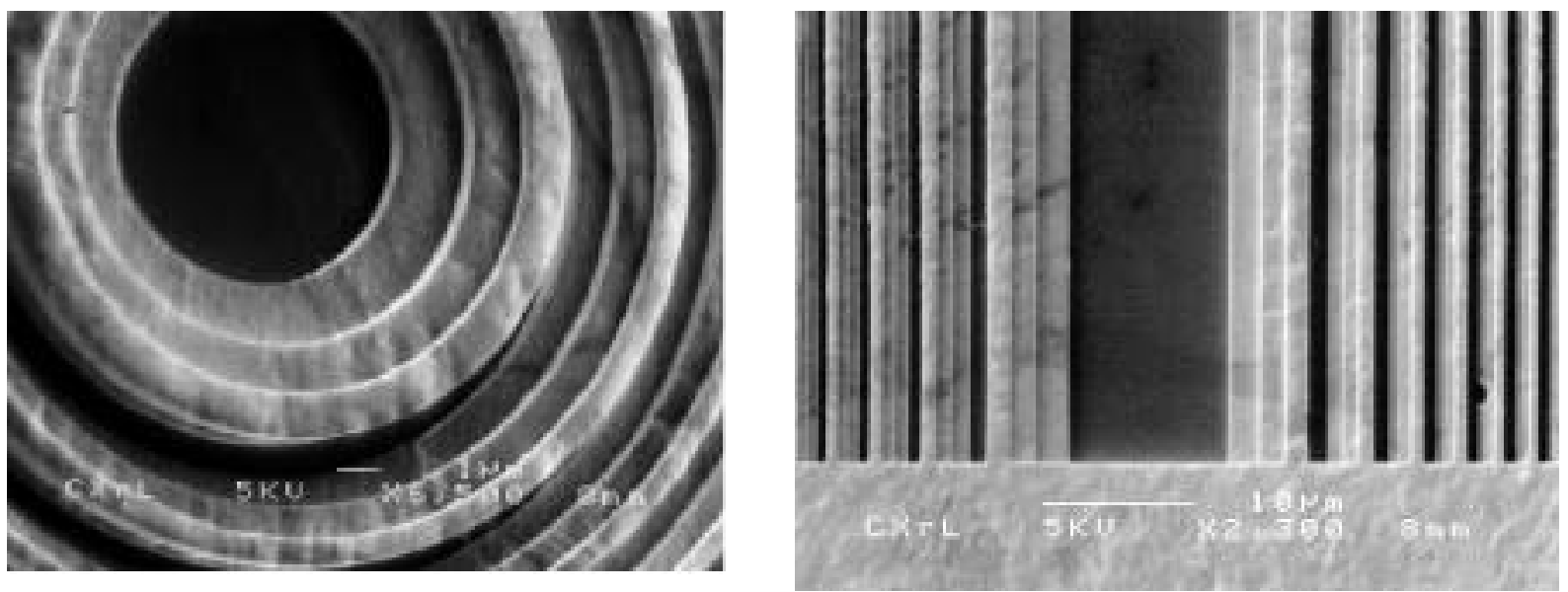

Fig. 5.47 Scanning electron micrograph of a circular (left) and a linear (right) zone plate with a staircase-like blazed zone profile.

importantly, this shows that by aligning multiple zone plates together it will be possible to use zone plates for much higher energies than was possible previously.

\section{X-ray Microfocusing Applications}

The development of microfocusing-based techniques is directly coupled to its applications. X-ray fluorescence microscopy (XFM), microdiffraction (XMD), microspectroscopy, microbeam small-angle scattering (XMSAS), fluorescence correlation spectroscopy (XFCS), and x-ray fluorescence tomography (XFT) have been developed and demonstrated in a series of experiments at the 2-ID-D station. These techniques have been applied to the following applications: (1) study of dynamics of colloidal systems using XFCS in collaboration with a scientist from Northern Illinois University and ANL MSD; (2) anticancer agents in collaboration with scientists from La Trobe University and the University of Melbourne (both in Australia); (3) trace elements distribution in $\mathrm{SiC}$ nuclear fuel shell using XFT in collaboration with scientists from Oak Ridge National Laboratory; (4) local molecular alignment within mesoscopic fibers of DNA-cationic membrane complexes using XMSAS in collaboration with scientists from the University of California, Santa Barbara; (5) study of an electro-absorption laser/modulator using XMD in collaboration with scientists from Lucent Technologies; (6) study of the plant-fungi relationship in contaminated environments using XFM in collaboration with scientists from ANL ER; (7) mapping of strain field and crystallographic phases near the edge of a ground steel component using XMD in collaboration with a scientist from Warner Lambert, Inc.; (8) electromigration study using XMD in collaboration with scientists from the University of Wisconsin; (9) study of functions of $\mathrm{Cu}$ in cells using XFM in collaboration with a scientist from MIT; (10) imaging of trace elements in bacteria using XFM in collaboration with scientists from ANL ER; (11) strain field mapping by XMD near a crack tip and measurement of single grains within a finite size specimen in collaboration with a scientist from Los 
Alamos National Laboratory; and (12) source identification of airborne pollutant particles using XFM in collaboration with a scientist from the Australian National Science and Technology Office. A brief description of some of these applications is provided in the following.

\section{X-ray Fluorescence Correlation Spectroscopy}

Photon correlation spectroscopy, which is used to study the dynamics of particles in fluids by measuring the fluctuations in the scattered and fluorescent intensity, is a wellestablished technique with visible light. With the advent of high-brilliance synchrotron radiation sources, such techniques have recently been extended to the $\mathrm{x}$-ray wavelength region by utilizing $\mathrm{x}$ ray beams possessing a high degree of spatial coherence and by studying the time fluctuations of the corresponding speckle patterns from the samples in question. In this present study, we have developed a related technique, $\mathrm{x}$-ray fluorescence correlation spectroscopy (XFCS), for elucidating the dynamics of single particles or clusters of particles (Wang, 1998). This method relies on an intense microfocused x-ray beam but does not require a coherent beam per se. A distinct advantage of this method is elemental specificity by using an energydispersive $\mathrm{x}$-ray detector. This method is particularly useful for studying both diffusive particle motion and flow in optically opaque systems for which methods employing visible light are not suited. As a demonstration of the method, the dynamics of gold and ferromagnetic colloidal particles and aggregates undergoing both diffusion and sedimentation in water have been studied by measuring the timeautocorrelation of the $\mathrm{x}$-ray fluorescence intensity from a small illuminated volume (Fig. 5.48). The dynamic parameters obtained are in excellent agreement with theoretical estimates and other measurements. This technique has recently been used to study the dynamics of red blood cells (RBC) in whole human blood, also shown in Fig. 5.48, as well as the thermal stability of RBCs and the interaction between the RBCs and foreign ions. In addition, we have demonstrated that the microfocused beam size and incidental effects of instrumental vibration can be determined independently and precisely by XFCS. The potential applications of XFCS include the study of the motion of biological macromolecules containing heavy atoms on or across membranes; the study of interdiffusion of atoms at interfaces between two species of materials; the study of the sedimentation or flow of colloidal particles in fluids containing polymers or surfactants which, we note, is unobservable by coherent $\mathrm{x}$-ray intensity fluctuation spectroscopy techniques.

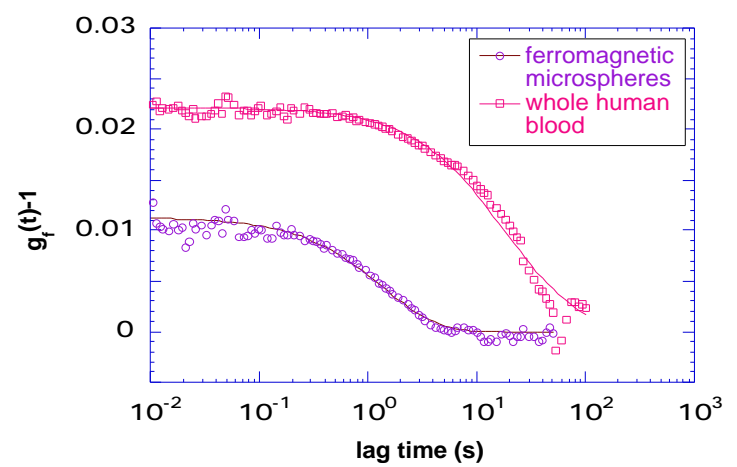

Fig. 5.48 Time autocorrelation functions obtained from the $\mathrm{Fe} \mathrm{K \alpha}$ fluorescence emission from two samples: ferro-magnetic microspheres, and red blood cells in whole human blood. The solid lines are the fits utilizing models describing both diffusion and sedimentation motions. 


\section{X-ray Imaging Studies of the Mycorrhizal Fungus-Plant Symbiosis}

Approximately $90 \%$ of the world's vascular plants, including the majority of all economic crops, belong to families that commonly have symbiotic associations with mycorrhizal fungi. While such associations are known to increase plant viability under low nutrient conditions, in some instances mycorrhizal fungi can also moderate toxicity effects in plants growing on soils containing elevated concentrations of heavy metals. Thus, an improved understanding of the plant-fungus relationship, particularly with respect to the uptake and regulation mechanisms for metals and micronutrients, is expected to have significant implications in both agriculture and the remediation and restoration of contaminated environments.

We have applied x-ray fluorescence microscopy to study the symbiotic relationship of Plantago lanceolata $L$. roots that have been infected by the arbuscular mycorrhizal fungus Glomus mosseae. The roots were kept in their natural hydrated state without any staining, thus avoiding any artifact due to sample preparation. The distributions of micronutrients $\mathrm{Mn}, \mathrm{Fe}, \mathrm{Cu}$, and $\mathrm{Zn}$ were recorded simultaneously with those of $\mathrm{P}, \mathrm{S}, \mathrm{K}, \mathrm{Ca}$, and $\mathrm{Ni}$, using a microfocused $\mathrm{x}$-ray beam of $1 \mu \mathrm{m} \times 3 \mu \mathrm{m}$. The elemental sensitivity is approximately $500 \mathrm{ppb}$, considerably better than that of either electron or proton microprobes. Typically, the fungal hyphae can be distinguished from root hairs because they branch and enter the root at multiple points. We found low concentration of $\mathrm{Mn}$ in the fungal hyphae, which agrees with the fact that mycorrhizal plants tend to have much less Mn than non-mycorrhizal plants. Also, the results (Fig. 5.49) indicate that Fe tends to be most concentrated on the edge of the root, perhaps reflecting the precipitation of $\mathrm{Fe}$ in this location. $\mathrm{Zn}$ seems to typically show up most strongly in the fungal hyphae and in the center of the root, most likely in the inner cortex where the proliferation of

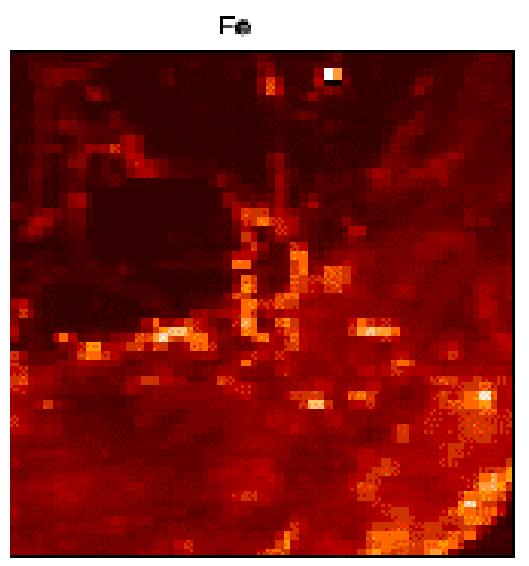

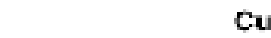

$\mathrm{Cu}$

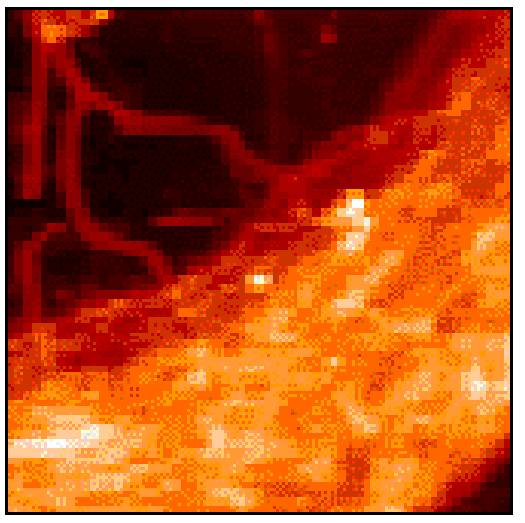

Llin.

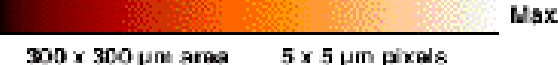

Zn

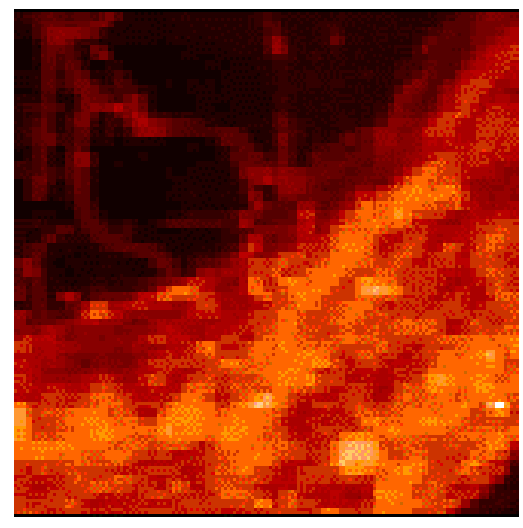

Wax

Fig. 5.49 Elemental distribution of $\mathrm{Fe}, \mathrm{Cu}$, and $\mathrm{Zn}$ in a plant root infected by mycorrhizal

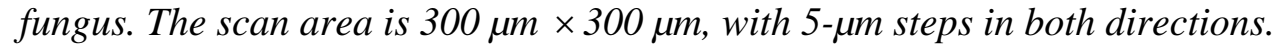


the fungus is typically the greatest. This suggests the use of $\mathrm{Zn}$ as a surrogate measure of mycorrhizal fungi in roots and additional work is underway to confirm this correlation. In addition, we recorded microXANES spectra of the Mn K-edge at selected positions within the hydrated sample, including on a single fungal hypha, to study the chemical state. Ninety percent of the Mn sampled by the X-ray beam is in the $\mathrm{Mn}^{+2}$ state, the soluble and most useful form to plants and fungi. This experiment clearly demonstrates that the microprobe is capable of providing high-quality microXANES measurement with micrometerscale resolution.

\section{Detection of Platinum Anticancer Agents in Ovarian Cancer Cells}

Cisplatin is one of the best anticancer agents for the treatment of testicular cancer, and is also used for a wide range of other tumors (e.g., ovarian, head and neck, lung). Cisplatin has a variety of dose-limiting side effects, including nephrotoxicity and neurotoxicity. Its analogue carboplatin has fewer severe side effects and is also in widespread use for treatment of similar tumor types. One of the major reasons for failure of a tumor to respond, or for a tumor to relapse when treated with cisplatin and carboplatin, is the presence of intrinsic (inherent) or acquired resistance, which was developed when the cancer was treated with cisplatin. If a cisplatin analogue was able to overcome this platinum resistance, cancer therapy could be substantially improved. There has been considerable interest to find derivatives of cisplatin that exhibit good activity against cisplatin-resistant cells. The development of these derivatives has been severely hampered by the lack of a clear understanding of the molecular processes involved in the development of this resistance, partially due to the lack of a tool that would allow one to quickly measure the interaction of the cisplatin or carboplatin derivatives with the cisplatin-resistant cells. Several resistance mechanisms have been identified in cisplatin-resistant cells in laboratory studies, including increased levels of cytosolic detoxifying, such as glutathione and metallothionein, enhanced repair of DNA damage, tolerance of cells to DNA damage, and decreased cellular or nuclear accumulation. However, it is not known which mechanism is the most important clinically. It is likely that a major advance in understanding this phenomenon can be gained by ascertaining how the subcellular distribution of cisplatin derivatives changes between resistant and sensitive cells.

The $\mathrm{x}$-ray microprobe at 2-ID-D was employed to map the spatial distribution of cisplatin and its derivatives in the resistant and sensitive cells using platinum M-line fluorescence. The platinum derivative used in this study is a drug known as Pt103. It has been selected as the lead compound for development of future compounds based on its good activity against platinum-resistant cell lines. Normal ovarian cancer cells 2008 and cisplatin-resistant cells C13 were treated with cisplatin and Pt103, respectively, for varied time periods for comparison of the effectiveness between anticancer agents, between sensitive and resistant cells, and among different treatment times.

The $\mathrm{x}$-ray beam was tuned to $11.7 \mathrm{keV}$ and was focused by a zone plate of $10-\mathrm{cm}$ focal length to a spot size of $0.2 \mu \mathrm{m} \times 0.5 \mu \mathrm{m}$. Figure 5.50 shows a platinum fluorescence image of a sectioned ( $1 \mu \mathrm{m}$ thick) cell treated with agent. The platinum distribution 


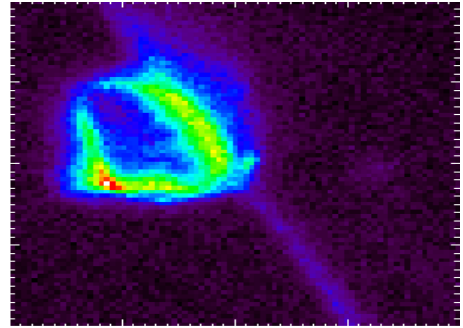

Fig. 5.50 Elemental distribution of $\mathrm{Pt}$ agent measured on a thin section (1 $\mu \mathrm{m}$ thick) of an ovarian cancer cell. The Pt distribution is very localized. The scan area was $40 \mu \mathrm{m} \times 40 \mu \mathrm{m}$.

is highly localized, which can provide insights into understanding the reaction of the cancer cells to the agent at a subcellular level. In addition, we measured the fluorescence signals obtained from individual whole cells, thereby studying the efficiencies of cisplatin and Pt103 to 2008 and $\mathrm{C} 13$ cells under different treatment times. The results show that the Pt103 derivative is five times more effective than cisplatin in platinum-resistant cells and is three times more effective in normal 2008 cancer cells after treatment for 8 and 24 hours. The effectiveness of both agents in 2008 and C13 cells increased as treatment time increased. For most of the cisplatin samples scanned, there was a more pronounced nuclear localization than for Pt103, which showed a more homogeneous distribution. The level of platinum sensitivity was several orders of magnitude higher than previous results obtained with the proton microprobe PIXE. This shows that a X-ray microprobe can provide a new tool for studying uptake of chemical agents at the cellular and subcellular levels.

\section{Measurement of Strain Field and Crystallographic Phases at the Edges of Steel Components}

The surface hardness and fatigue resistance of the edges of a ground component made of martensitic stainless steel are a function of the amount of retained austenite, its residual stress, and its carbide parameters. The cold work induced by grinding causes a phase transformation from austenite to martensite, which is associated with a volume expansion and increased residual compressive stress. The residual stress at the ground surface of such components has not been measured successfully before because of the large beam size of conventional x-ray diffraction sources. In order to optimize the performance of these components, it is essential to establish a method to measure the residual stress at the ground surface on micrometer length-scales, and to understand how the residual stress changes are related to the grinding process.

At beamline 2-ID-D/E, we used an x-ray microdiffraction technique to characterize the lattice strain distribution in thin martensitic stainless steel components as a function of their grinding parameters. The lattice strain of the samples was determined from CCD camera images of the $x$-ray microdiffraction pattern resulting from the martensite (110) and austenite (111) planes. Micrometer-size $(0.5 \mu \mathrm{m} \times 3 \mu \mathrm{m})$ areas near the edges of the samples were illuminated with a 12-keV x-ray microbeam formed with a zone plate lens. The samples were aligned such that the scattering vector was 
along their edges. The lattice strain of the martensite (110) plane was measured as a function of the distance from the illuminated area to the sample edge. During each CCD exposure of the sample diffraction pattern, at a given distance from its edge, the sample was scanned along the horizontal direction to enhance the statistics of the measurement.

Figure 5.51 shows two CCD images of the diffraction patterns near the edge of one sample. The pattern obtained $40 \mu \mathrm{m}$ from the edge shows diffraction from phases of both austenite and martensite. Only diffraction from martensite phase was observed $1 \mu \mathrm{m}$ from the edge, indicating that a phase transformation from austenite to martensite took place during the grinding process. We also observed a smaller lattice constant along and near to the edge, suggesting a compressive residual stress resulted from the phase transformation. We performed $\mathrm{x}$-ray microdiffraction measurements at distances of 1-600 $\mu \mathrm{m}$ from the edges of several samples containing $12 \%$ and $20 \%$ initial retained austenite to correlate the amount of retained austenite to the residual stress after grinding. The results indicate that the samples with a higher $(20 \%)$ retained austenite not only had a higher residual compressive stress than the ones with lower (12\%) retained austenite, but also had a higher retained austenite after grinding. In the future we hope to determine the optimum residual stress and retained austenite combination to maximize the hardness and fatigue resistance of the ground surface of such components.

\section{$1 \mu \mathrm{m}$ from the tip}

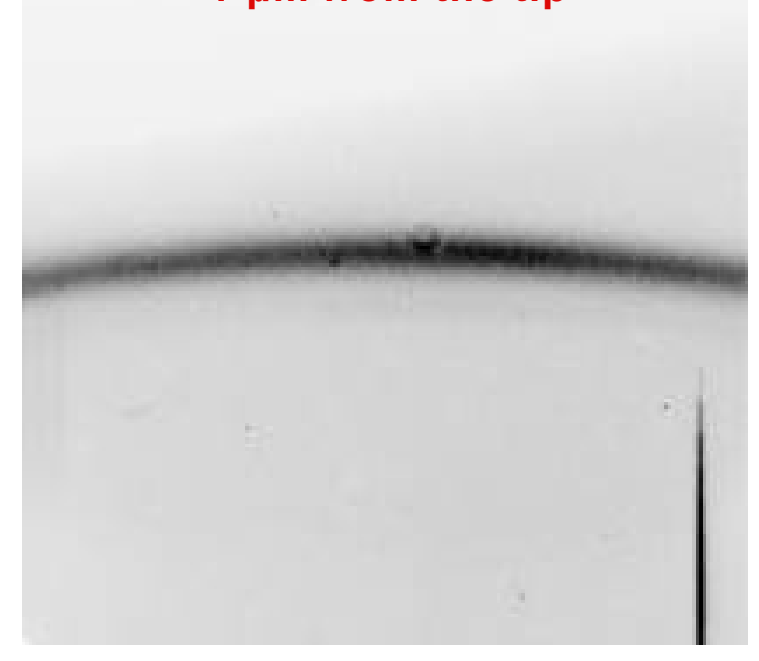

$40 \mu \mathrm{m}$ from the tip

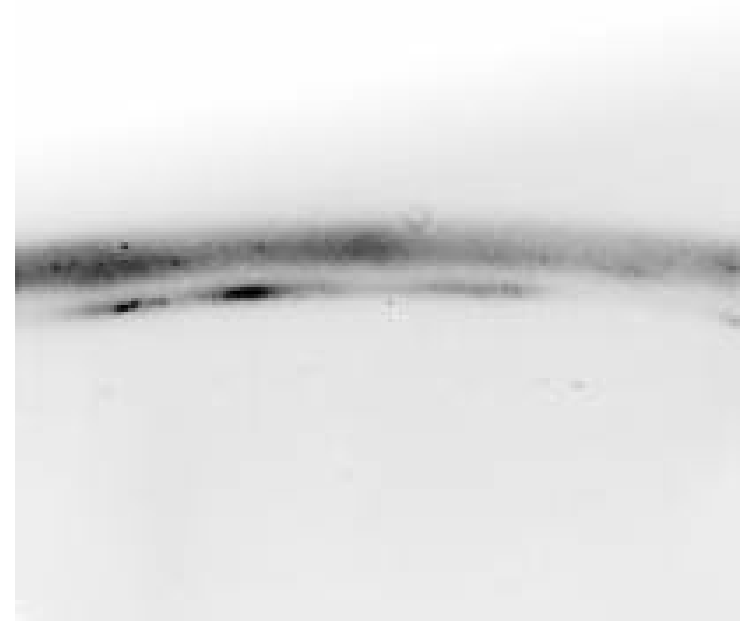

Fig. 5.51 Microdiffraction patterns taken at the tip of a thin stainless steel component. 


\section{Strain Measurement on a Single Grain Using Microdiffraction}

An understanding of fracture mechanisms is important in evaluating the aging of materials. Conventionally, neutrons and $\mathrm{x}$-rays have been utilized to map the residual stress/strain, mosacity and crystallite size, etc., but they were limited to bigger gauge volumes. We developed a new method to examine much smaller gauge volumes $\left(10^{-3}\right.$ to $10^{-5} \mathrm{~mm}^{3}$ ) and with higher spatial resolution $(1 \mu \mathrm{m})$ unlike the neutron measurements. These new results could lead to doing crystallography on single grains and will help to understand micro-stresses and strains in materials.
The microdiffraction measurements were carried out on beryllium Compact Tension (CT) specimens of differing textures in the critical region (i.e., in and around the notch point) at room temperature by using 11.0-keV x-rays at 2-ID-D beamline. The Be sample with a load cell is mounted on a stage that has translational and rotational manipulation. The load is changed manually, and then the beam is aligned at the notch with a x-ray phase contrast image. A CCD detector is used to record the diffraction pattern from the sample. The diffraction peak from each individual grain can be tracked when the load is changed. Such a case is shown in Fig. 5.52, where the diffraction peak position (along the azimuthal direction) is plotted for

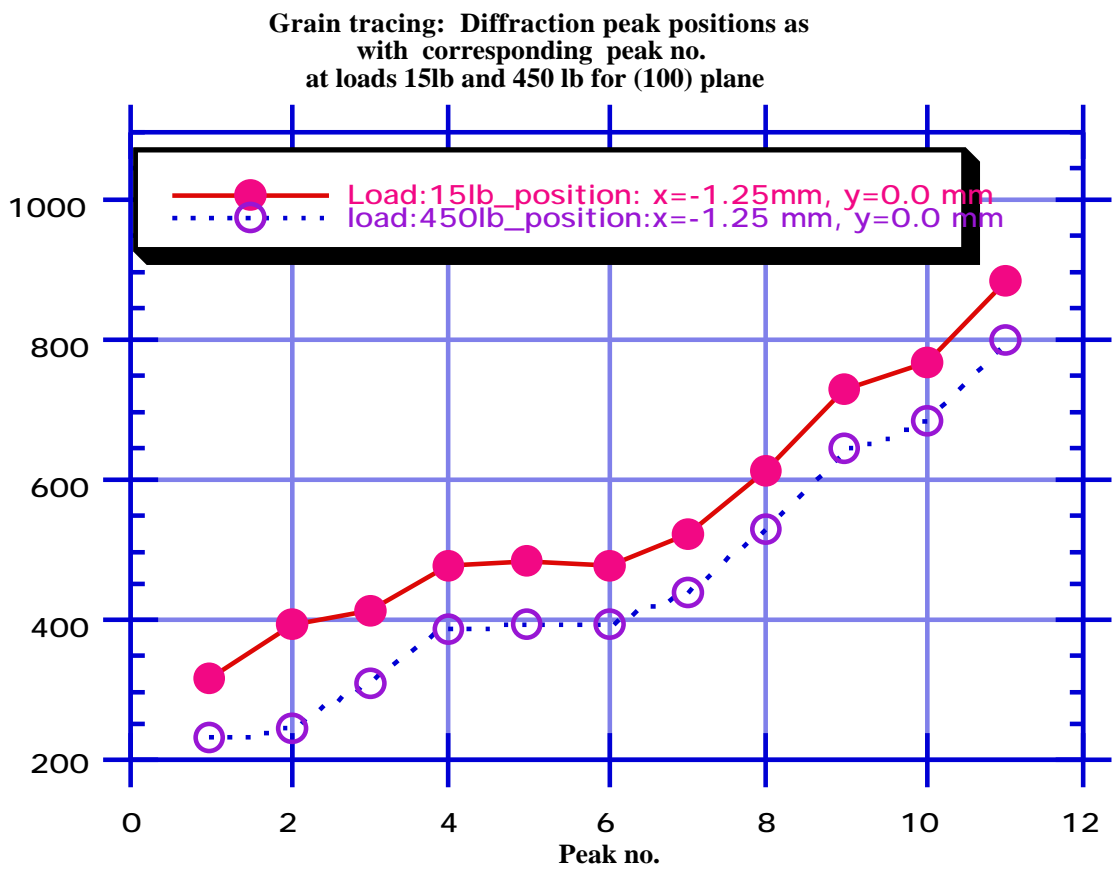

Fig. 5.52 The azimuthal locations of 11 diffraction spots recorded with the CCD camera under loads of 6.8 and $205 \mathrm{~kg}$. The fact that the two curves have the same shape suggests that the same 11 grains were diffracting under both loading conditions. 
11 diffraction spots when the load was increased from 6.8 to $205 \mathrm{~kg}$. The fact that the relative peak position does not change indicates that the same grain is diffracting in both cases, allowing us to track the strain of individual grains during the stress measurement, which will provide valuable data previously unavailable for simulation and finite element calculation. The result shows that the microstrain increases at the notch point for the $\mathrm{Be}(100)$ reflection. At $1 \mathrm{~mm}$ away from the notch, the microstrain did not recover fully but is smaller than that at the notch. We also determined that the mosaic spread increases at the notch point and is large with larger load

\subsubsection{Sector 3}

\section{Developments at the 3-ID Beamline}

During the past year, the remaining two stations of the 3-ID beamline were completed. Stations 3-ID-C and 3-ID-D became operational in February 1998, opening the way for independent investigators by February 1999. During the year, there were some important upgrades to the beamline including the installation of a 5-mm internal gap ID vacuum chamber, which allows us to have the undulator between 6-30 keV with no spectral gap. Beamline 3-ID became the first in which the undulator gap can be reduced to $8.5 \mathrm{~mm}$ (see section 5.1.5 for details). Additional shielding to the white beam shutter and FOE has been completed, and the undulator gap can now be reduced to its minimum value of $8.5 \mathrm{~mm}$. Another important change was the installation of diamonds as high-heat-load monochromator crystals. Studies conducted at an undulator gap of $8.5 \mathrm{~mm}$ indicated no adverse affects on beam divergence. This water-cooled system has been in place since May 1997, and the first year of experience is satisfactory.

During the year, we also commissioned the collimating and focusing double-groove mirror and demonstrated that the beam divergence can be reduced from $17 \times$ $40 \mu \mathrm{rad}$ (vertical and horizontal) to $11 \times$ $20 \mu \mathrm{rad}$. When testing, the reflectivity of the mirror is better than $97 \%$ in the $7-24 \mathrm{keV}$ range. The focal spot size in the 3-ID-C station has been measured to be less than $450 \mu \mathrm{m}$ horizontal and $300 \mu \mathrm{m}$ vertical after the high-energy-resolution monochromator.

The general approach followed when conducting high-energy-resolution experimentation at beamline 3-ID was to unify the monochromatization process and develop different styles of analyzers. The inelastic x-ray scattering setup using an "inline" monochromator and curved crystal backscattering is shown in Fig. 5.53 (a), and the configuration for inelastic nuclear resonant scattering is shown in Fig. 5.53 (b).

The underlying principle in high-energyresolution monochromatization is to employ an optimum combination of asymmetrically cut single-crystal reflections to create the desired energy bandpass, angular acceptance, and overall efficiency or throughput. Several different software packages have been developed to optimize certain parameters, such as the choice of reflection planes, the degree of asymmetry, and energy resolution (Toellner, 1996). These programs include calculation of modified DuMond diagrams (Mooney, 1990); accurate 3-D (angle-energyreflectivity) throughput calculations of multiple-crystal systems, including the source divergence and bandpass (Toellner, 1998); and the complete treatment of 

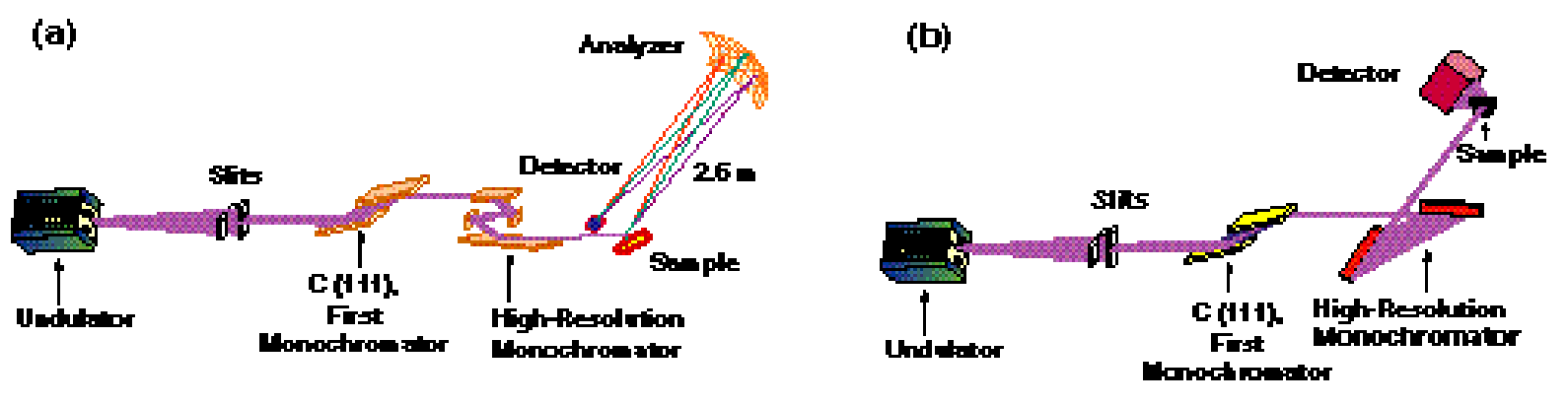

Fig. 5.53 The schematic layout for high-energy-resolution inelastic $x$-ray scattering as implemented at the 3-ID beamline of SRI-CAT. (a) The inelastic $x$-ray spectrometer that consists of an "in-line," nested, 2-channel-cut design high-resolution monochromator followed by a curved crystal analyzer. (b) The inelastic nuclear resonant scattering spectrometer that consists of two flat-crystal monochromators followed by a fast detector with nanosecond resolution for time discrimination. An in-line monochromator, as shown in (a), is also available for several isotopes for coherent or incoherent nuclear resonant scattering.

multibeam excitations in single crystals, including exact backscattering (Colella, 1998).

As for new monochromator components, we have completed a 1-meV-resolution silicon monochromator at $21.5 \mathrm{keV}$, and 3.4-meVresolution monochromator at $24 \mathrm{keV}$, and a 2-meV, 0.8-meV, and 0.6-meV resolution monochromator at $14.4 \mathrm{keV}$. It is worthwhile to note that the energy bandpass of the 0.66-meV-resolution monochromator at $14.413 \mathrm{keV}$ with $3 \times 108$ photons/sec represents the highest photon flux with the lowest $\Delta \mathrm{E} / \mathrm{E}$ achieved so far, using the flatcrystal concept described previously (Toellner, 1996; Toellner et al., 1997; Chumakov et al., 1996a). The monochromators at 21.5 and $14.4 \mathrm{keV}$ can be used both for inelastic x-ray scattering with a backscattering analyzer and for nuclear resonant scattering purposes.

Analysis of the energy spectrum of inelastically scattered x-rays with sufficient resolution and efficiency continues to be a challenge. The conflicting aspects of this method stem from the divergent nature of the scattered $x$-rays and the limited angular acceptance of crystals used as analyzers. The problem becomes particularly serious when the required resolution drops below $10 \mathrm{meV}$ in the 6-30 $\mathrm{keV}$ range. The established method, originally introduced in the early 1980s (Dorner et al., 1986), involves the use of near-backscattering geometry from high-order Bragg reflections. In order to improve the total solid angle subtended by the analyzer, the thin crystal would be bent to a spherical shape with several-meters radius of curvature. This, accompanied by several different procedures to reduce or eliminate bending stress (Burkel et al., 1989; Verbeni et al., 1996), provides a reasonable solution to an immediate problem. We have developed a new procedure for use in preparing $10-\mathrm{cm}-$ diameter diced analyzers comprising approximately 8,000 crystals. With respect to earlier methods, we take a new approach to reducing the strain on the curved analyzer. A Pyrex wafer was used as substrate to position the 8,000 crystals. Then, the Pyrex-epoxy-silicon "sandwich" is pressed into a concave substrate with a 
2.6-m bending radius (Schwoerer-Böhning et al., 1997).

\section{Resonant and Non-Resonant Inelastic Scattering Program}

Inelastic $\mathrm{x}$-ray scattering is a powerful and important tool for the study of collective excitations in condensed matter systems. The technique measures the dynamic structure factor, which leads to an understanding of electron or atomic correlations in space and time. At moderate energy resolutions (few hundred $\mathrm{meV}$ ), electron correlations can be studied in metallic systems, strongly correlated electron systems, etc.

At ultrahigh energy resolution (a few $\mu \mathrm{eV}$ to $\mathrm{meV}$ ), the dynamic correlations of ion cores (phonons) can be studied using the inelastic $\mathrm{x}$-ray scattering technique in systems where traditional methods, such as neutron scattering, may be less applicable. These include ultrahigh-resolution nuclear $\mathrm{x}$-ray inelastic scattering to derive partial phonon density of states from disordered systems, thin films, nano-particles, etc. The ultrahighenergy-resolution x-ray experiments will also focus on the dynamics in glasses and liquids.

The use of backscattering geometry provides an opportunity to measure phonon frequency-momentum dispersion relations along high-symmetry crystallographic directions from single-crystal samples. The inelastic spectrometer in the 3-ID-C station has been used to measure spatial phonon dispersion in diamond with an overall instrument resolution of $9.2 \mathrm{meV}$. Diamond has unusual static properties when compared to other class IV tetrahedral semiconductors.
Lattice dynamical characteristics, such as phonon dispersion and thermal expansion, are also distinctive. Additionally, the occurrence of the most energetic phonons away from the Brillouin zone center is peculiar to diamond. A necessary condition to have such an overbending is to have sufficiently large second-nearest-neighbor force constants. Figure 5.54 shows an example of a phonon dispersion in diamond measured along the [111] direction, the complete set of dispersion data is shown in Fig. 5.55. The expected overbending in the most energetic LO branch along both $\Gamma-\mathrm{X}$ $(=\Delta)$ and $\Gamma-\mathrm{L}(=\Lambda)$ and the elliptically polarized $\Sigma_{3}$ branch along $\Gamma-\mathrm{K} \quad(=\Sigma)$ received special attention in our measurements. Overbending in all three directions has been invoked to explain an extraordinary peak in the two-Raman spectrum. The overbending from our measurements is seen only along $\Delta$ (Schwoerer-Böhning et al., 1998).

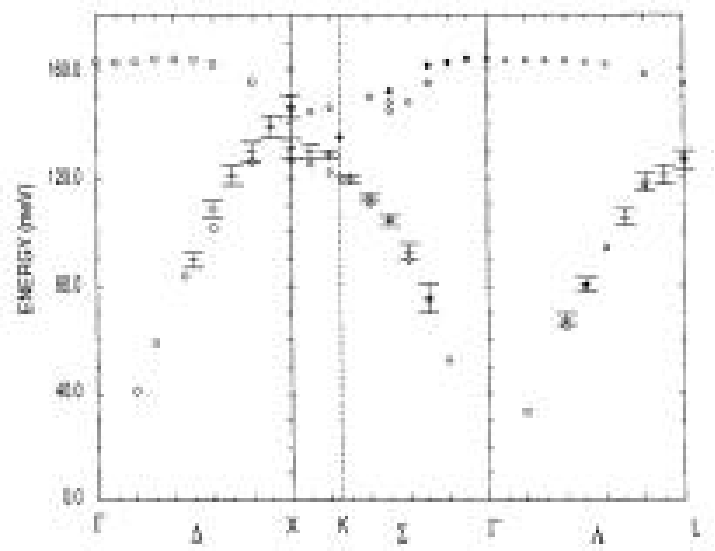

Fig. 5.54 Longitudinal phonon branches along $\Delta, \Sigma$, and $\Lambda$. Symbols without error bars are high-resolution inelastic $x$-ray scattering data; symbols with error bars were obtained by inelastic neutron scattering. 


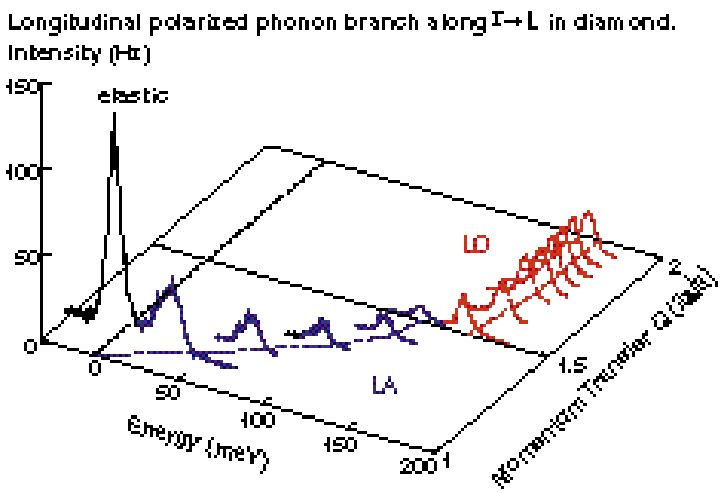

Fig. 5.55 Spectra at different momentum transfers representing longitudinal acoustic and optic modes in diamond along the [111] direction (L). The measurements were carried out using the Si (777) reflection of the analyzer at a Bragg angle of $89.97^{\circ}$ with a total energy resolution of $7.5 \mathrm{meV}$. These data prove that there is no measurable overbending in the optical mode as predicted by ab initio calculations.

Resonant inelastic scattering from insulating cuprates with $0.8-\mathrm{eV}$ resolution was demonstrated, and the high flux was used to acquire systematic data, which were essential for understanding the nature of the resonance process. Also, the energies of various electronic states near the Fermi surface were measured. Given the experimental challenges in achieving the high energy resolution required to measure exciton dispersion, we instead used the fact that the coupling mechanism between the electronic charge and photons is highly polarization dependent to deduce information on the symmetry of the highenergy excitations we had seen earlier. This involved measuring the detailed resonance profiles for a variety of experimental geometries using (001), (100) and (110) cut $\mathrm{Sr}_{2} \mathrm{CuO}_{2} \mathrm{Cl}_{2}$ single crystals. It was possible to deduce that the high-energy transitions we had seen are highly polarized in the b1g (i.e., $d_{x 2-y 2}$ channel and probably correspond to transitions from the b1g ground state to a broad a1g continuum that comes from an antibonding combination of the $\mathrm{Cu} 3 \mathrm{~d}_{\mathrm{x} 2-\mathrm{y} 2}$ orbital and the surrounding $\mathrm{O} p(\mathrm{~s})$ orbitals. Complete assessment of these measurements is underway.

Figure 5.56 demonstrates the capability for studying phonons in transition metals. To study electronic energy levels near the Fermi level, a backscattering $\mathrm{Ge}$ analyzer with $100-\mathrm{meV}$ resolution at $9 \mathrm{keV}$ has been tested with direct incident beam of $700-\mathrm{meV}$ bandpass. This spectrometer has been used to study correlated electron systems in rareearth cuprates.

The inelastic scattering technique described above requires the use of single crystals. In order to study polycrystalline materials, powder samples, and, in particular, thin films, alternative methods must be developed. One such alternative is the use of nuclear resonance as an energy analysis technique. This approach was introduced in

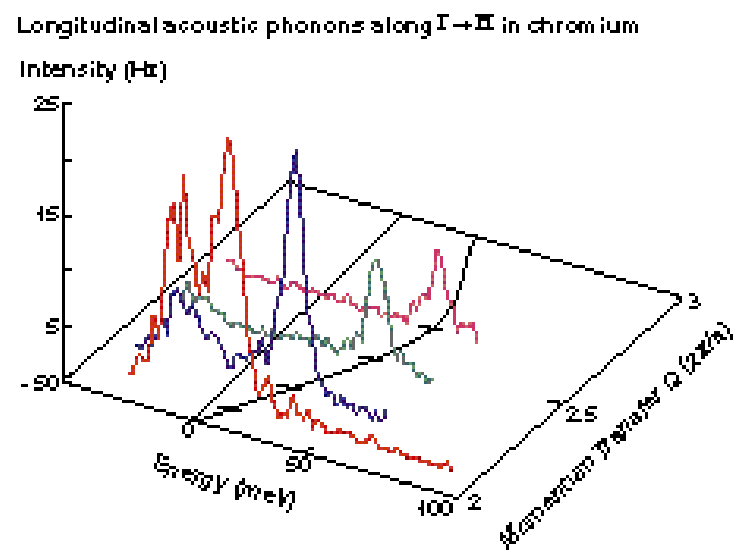

Fig. 5.56 Studies of phonons in chromium, demonstrating the performance of the instrument for high-resolution inelastic $x$-ray scattering. 
1994 (Seto et al., 1995; Sturhahn et al., 1995; Chumakov et al., 1996b), and it relies on tunable high-energy-resolution monochromators and the presence of suitable isotopes in the sample under analysis. This technique provides unequaled capability in terms of measuring partial phonon density of states in any medium, solid or liquid, crystalline or amorphous, bulk or thin films-even monolayers at an interface. It also allows extraction of phonon density of states directly from the data without the knowledge of crystal structure. During the 1997-98 period, the inelastic nuclear resonant scattering studies continued using $\mathrm{Fe}$ and have been extended to $\mathrm{Sn}$ and $\mathrm{Eu}$ isotopes. There were many experiments in thin films, monolayers at interfaces, samples under high pressure, biological samples at low temperatures and amorphous materials. For example, we have demonstrated that this method can be utilized to study the effect of reduced dimensionality on vibrational modes. In this instance, we measured phonon density of states of nanocrystalline iron (Fultz et al., 1997).

A brief description is provided below of the vibrational behavior of $\mathrm{Fe} / \mathrm{Cr}$ multilayered systems. For this purpose, it was envisaged to measure the vibrational density of states (VDOS) at different locations in a multilayer through the elemental selectivity inherent with this technique. In recent literature, the method of obtaining the phonon spectrum of a system involving nuclear resonance as an energy analyzer is referred to as PHOENIX (PHOnon Excitation by Nuclear Inelastic absorption of X-rays). In this study, monolayers of ${ }^{57} \mathrm{Fe}$ were placed either in the center of an iron layer or at a $\mathrm{Cr}-\mathrm{Fe}$ interface. The iron layers themselves consist of ${ }^{56} \mathrm{Fe}$ to suppress any unwanted effects due to the $2 \%$ abundance of ${ }^{57} \mathrm{Fe}$ in natural iron. In this experiment, a newly developed "nested monochromator" with an energy resolution of $2.1 \mathrm{meV}$ was used. This device represents considerable improvement in energy resolution compared to the previous 5.5-meV monochromator while maintaining ample photon flux. In addition, incident and exit beams remain parallel, which is quite advantageous in thin-film experiments of this type. The samples were prepared in Duisburg by evaporation under UHV conditions. A properly cleaned $\mathrm{MgO}$ substrate was coated with a $50 \AA$ Cr buffer layer. The deposition sequences are then $200 x\left[{ }^{57} \mathrm{Fe}(1 \AA) / 56 \mathrm{Fe}(11.5 \AA) / \mathrm{Cr}(11.5 \AA)\right]$ (probe layer at the interface, type $\mathrm{I}$ ), $200 x\left[{ }^{56} \mathrm{Fe}(5.7 \AA) / 57 \mathrm{Fe}(1 \AA) / 56 \mathrm{Fe}(5.7 \AA) / \mathrm{Cr}\right.$ $(11.5 \AA)]$ (probe layer at the center, type $\mathrm{C}$ ). All of the six provided samples ( 2 of type $C$ and 4 of type I) were investigated, and the VDOS were determined successfully. The VDOS of the type C samples and bulk-iron (alpha phase) are virtually identical. However, when the ${ }^{57} \mathrm{Fe}$-probe layer is placed at the interface (type I samples), significant changes in the VDOS are observed. High-energy phonon modes (35 meV, supposedly near the Brillouin zone) are clearly suppressed. On the other hand, the density of medium-energy modes, particularly around $23 \mathrm{meV}$, is visibly enhanced. The experimental results clearly demonstrate the validity of the PHOENIX technique when applied to vibrational dynamics of thin films. The illuminated amount of probe material $\left({ }^{57} \mathrm{Fe}\right)$ was only about 5 micrograms. Similar measurements were taken in $\mathrm{Fe} / \mathrm{Au}$ multilayer systems with varying thicknesses of $\mathrm{Fe}$ layers to investigate the effect of the presence of heavy atoms. Partial phonon density of states of $\mathrm{Fe}$ in this multilayer system is shown in Fig. 5.57. 


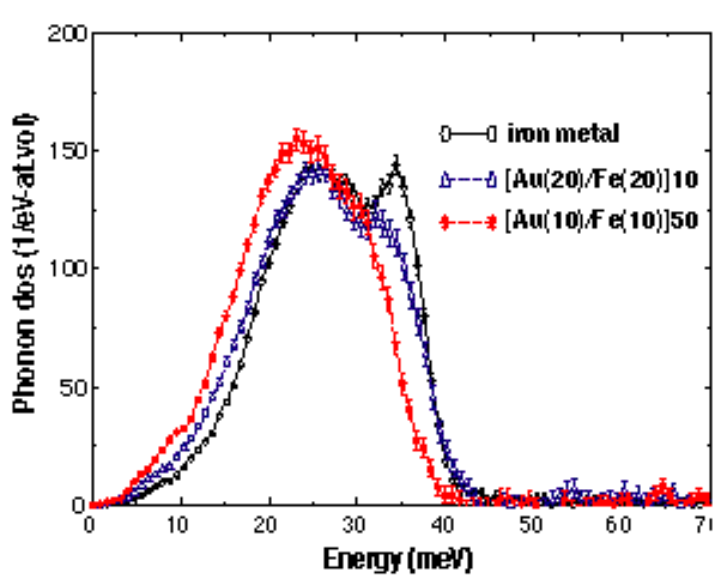

Fig. 5.57 Phonon density of states of Fe in an $\mathrm{Fe} / \mathrm{Au}$ multilayer with varying thicknesses. As the layer thickness decreases, the softer modes below $25 \mathrm{meV}$ appear to gain. Also note the disappearance of the longitudinal acoustic mode at $36 \mathrm{meV}$. Similar trends were observed with decreasing thickness in pure iron films. (Work in progress, in collaboration with $S$. Bader of Argonne National Laboratory.)

The phonon density of states of Sn metal under high pressure has been measured using inelastic nuclear resonant scattering. The pressure calibration of the diamond anvil was done using sector 13, GEO-CARS facilities. We used 23.87-keV x-rays both to excite the ${ }^{119} \mathrm{Sn}$ nuclear resonance and to observe the delayed signal. Two specially designed APD detectors enabled a count rate of a few $\mathrm{Hz}$, which was sufficient to record three spectra in 3 days at $8,15,20 \mathrm{GPa}$ pressures. The total volume of sample illuminated was $0.05 \mathrm{~mm}^{3}$. This was also the first time we have employed a third asymmetric crystal to reduce the vertical beam size, so the energy scans required coordinated motion of all three crystals. The energy resolution for this measurement was $3.7 \mathrm{meV}$. This was a first demonstration of the feasibility of phonon density of states measurements under high pressure using
PHOENIX. Phase transitions under high pressure will be the focus of future investigations.

Soon other users with systematic research programs can begin their work at beamline 3-ID. In preparation, we have completed a data acquisition system in the lab for users to try to learn our beamline system motion control and data acquisition system. Also, some critical data analysis software was developed to allow users to extract phonon density of states from experimental data.

\subsubsection{Sector 4}

\section{Introduction}

In 1997, it was decided to extend and enhance the capabilities of SRI-CAT by building a new sector whose primary purpose will be to exploit the polarization properties of radiation, an area that other CATs have limited plans to pursue.

The objective of this sector is to develop instrumentation and techniques that will utilize the high brilliance, variably polarized x-ray beams produced from two APS undulators and will also perform high-heatload testing and diagnostics for Phase-2 FE and optical components. Two separate branch lines covering the "soft" (0.5 $3 \mathrm{keV}$ ) and hard (>3 keV) x-ray regions are planned. One of the key components for the new sector will be a circularly polarized soft $\mathrm{x}$-ray undulator. (The CPU is described in detail in section 5.1.3.) This is necessary because manipulation of beam polarization is not possible at energies between 1 and $2 \mathrm{keV}$ using optical phase retarders. In the hard $\mathrm{x}$-ray region, undulator A will be used 
with crystal optics, such as transmission Bragg and Bragg-Laue phase retarders.

Transferring the hard $\mathrm{x}$-ray polarization programs from sector 1 and the soft x-ray spectroscopy program from sector 2 will allow for improved sharing of beam time among all four SRI-CAT sectors. It will also give a clearer definition of the SRI-CAT sector structure. Sector 1 will specialize in high-energy x-ray optics development and time-resolved experiments, sector 2 will be the imaging and coherence techniques sector, sector 3 will remain as is: $\mathrm{meV}$ and nano-eV techniques, and sector 4 will become a polarization techniques sector with capabilities for testing components that have to withstand high heat loads. Another important aspect is that SRI-CAT will be able to make efficient use of the financial contributions from the X-ray Physics Group and the Australian Group, giving them and outside users improved accessibility to all of the SRI-CAT scientific resources.

The three major programs targeted for this new sector are:

1. Development of $0.4-3 \mathrm{keV}$ instrumentation for high-spectral-resolution/ highphoton-flux polarization-dependent spectroscopy programs. This will be achieved with an elliptically polarized undulator and a polarization-preserving grazing-incidence monochromator. The scientific program will cover magnetic circular dichroism (MCD), resonant magnetic scattering, spin-resolved photoemission, and x-ray fluorescence. An important aspect of this program is that this energy range covers the transition metal and rare earth $\mathrm{L}$ and $\mathrm{M}$ edges, respectively, as well as numerous important elements, such as the $\mathrm{O} \mathrm{K}, \mathrm{Cu}$ L, and Si K edges.
2. Development of polarizing optics and techniques for the $3-100 \mathrm{keV}$-ray energy range. This program will use a plane-polarized x-ray beam from undulator A together with polarizationmanipulating crystal optics. The scientific program will cover resonant and non-resonant magnetic scattering, magnetic circular dichroism, magnetic reflectivity, and magnetic Compton scattering.

3. Development of high-heat-load FE and optical components for use with storage ring currents up to $300 \mathrm{~mA}$. This program will use an experimental chamber as close to the shield wall as possible for thermal cycling of $\mathrm{FE}$ and beamline components.

In order to accomplish the objectives of the three programs outlined above, the spectral requirements outlined in Table 5.6 must be met.

In order to provide $\mathrm{x}$-rays from $0.4-100 \mathrm{keV}$ with high brilliance, two undulators will be installed in the sector 4 straight section. Both devices will be $2.4 \mathrm{~m}$ long. The hard $\mathrm{x}$-ray range will be covered using undulator A. A custom 12.8-cm-period CPU will primarily be used for the soft x-ray range. Figure 5.6 shows the brilliance range of the soft x-ray device in the linear polarization mode, together with a 5.0-cm-period elliptical device planned for the Advanced Light Source. The 12.8-cm device has been described in section 5.1.3 of this report.

A key innovation of this beamline was the proposed operation of two undulators simultaneously, supplying radiation to two independent branch lines, one for hard x-ray experiments and the other for soft $\mathrm{x}$-ray 
Table 5.6 Spectral Requirements for the Scientific Programs in Stations 4-ID-A (FOE), 4-ID-B, and 4-ID-C

\begin{tabular}{|l|c|c|c|}
\hline & $\begin{array}{c}4-I D-A \\
\text { (FOE) }\end{array}$ & 4-ID-B & 4-ID-C \\
\hline Spectral Range $(\mathrm{keV})$ & - & $3-100$ & $0.4-3.5$ \\
\hline Resolving Power & - & 10,000 & $2,000-12,000$ \\
\hline Beam Size @ Station $(\mathrm{mm})$ & 3 & $0.2-3$ & 1 \\
\hline Flux /0.1\% BW & - & $10^{14}$ & $10^{13}$ \\
\hline Source Polarization & - & $\mathrm{H}$ & $\mathrm{H}, \mathrm{V}, \mathrm{LCP}, \mathrm{RCP}$ \\
\hline Total Power $(\mathrm{kW})$ & 6 & & \\
\hline Power Density $\left(\mathrm{W} / \mathrm{mm}^{2}\right)$ & 230 & & \\
\hline
\end{tabular}

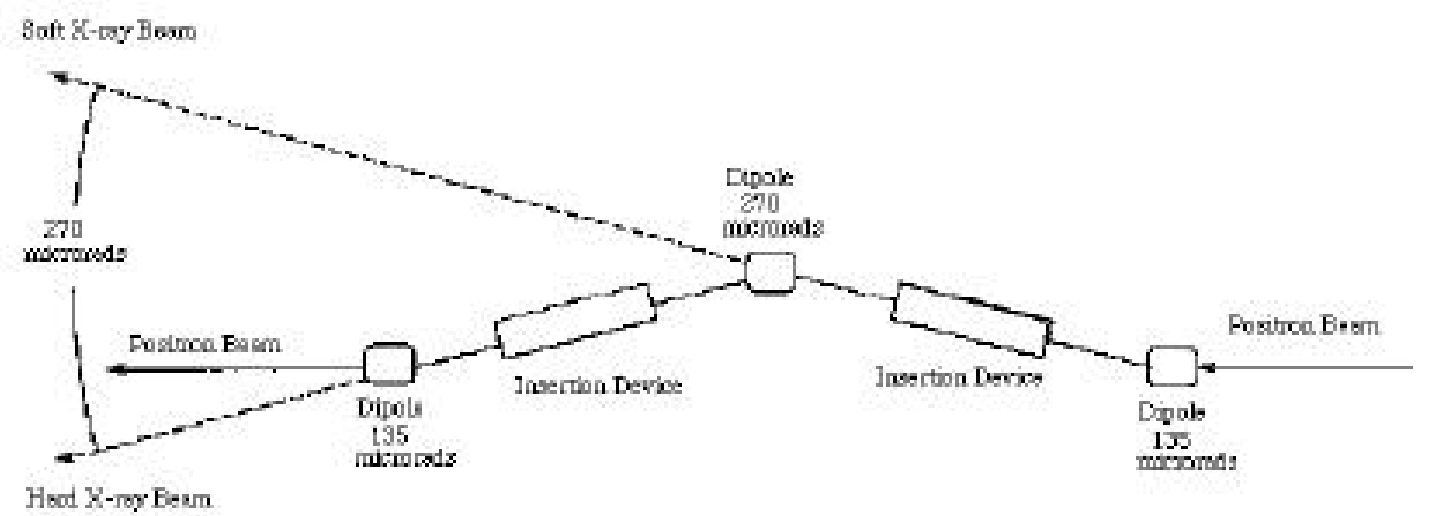

Fig. 5.58 Sector 4 insertion device plan.

studies. The beams from the two undulators will be spatially separated by introducing an angular deviation of the positron beam between the devices (Fig. 5.58). An initial estimate requires an $8-\mathrm{mm}$ beam separation in the FOE at approximately 30 meters from the center of the straight section. This will require a dipole electromagnet sufficient to steer the beam through 267 microradians, which is technically achievable (Decker, 1998).

\section{Layout of the Sector}

The ID beamline consists of two branch lines that share the same first optical enclosure (FOE), 4-ID-A. The optical design of this sector is based on eight considerations:

1. Spatially separated undulator beams for simultaneous operation of both branch lines 
2. Access to white beam (undulator A) directly adjacent to the shield wall for thermal loading tests

3. A windowless front end for access to soft x-ray energies

4. Access to white beam in a hutch beyond the FOE

5. A clear path beyond the white-beam hutch for possible extension of the hard $\mathrm{x}$-ray branch line

6. Use of a grazing-incidence soft x-ray monochromator for presentation of the soft $\mathrm{x}$-ray source polarization

7. Simple vacuum mechanical design for minimum downtime of both lines

8. Maximum utilization of available floor space

\section{Layout of the ID Beamline}

The ID beamline is split into two branch lines to optimize the utilization of the 0.5-30 $\mathrm{keV}$ undulator radiation (Fig. 5.58). The rationale for this branching is described in the first part of this section. The ID beamline is divided into three functional parts. The three parts are the first optics enclosure and the two branch lines, which are described separately below.

\section{Beamline Branching}

Our plan is to branch the two end stations, 4-ID-B and 4-ID-C, by spatially separating the undulator beams. The outboard (hard $\mathrm{x}$-ray) beam will pass through the FOE undeviated to the hard $\mathrm{x}$-ray white-beam station (4-ID-B). The inboard (soft x-ray) beam will be deflected outward to branch line 4-ID-C using a pair of horizontally deflecting mirrors. (See Fig. 5.17 for a layout of the beamline.) The incidence angle onto each mirror is $1.25^{\circ}$ giving a total deflection of $5^{\circ}$.

Major considerations for beam branching include:

- the compatibility of beamline optical components that are required for the different scientific programs in terms of polarization preservation, monochromator resolving power in the required energy range, mirror focusing requirements and monochromator dispersion plane requirements; and

- the engineering and construction of these optical components, thermal management of these optical devices, together with other aspects, such as beamline vacuum and radiationshielding requirements, and overall operation of the two branches.

The hard x-ray branch line is designed for polarization manipulation in the $3-100 \mathrm{keV}$ $\mathrm{X}$-ray energy range. This will be achieved by using crystal optics following the DCM.

The soft x-ray branch line is designed to preserve the source polarization properties and to deliver an x-ray beam of high energy resolution in the $0.4-3.5 \mathrm{keV}$ energy spectral region. This branch will be used for highresolution polarization-dependent spectroscopy. 


\section{Beamline Component Test Facility with Movable Mask}

The sector 4 FOE will also accommodate a 1.75-m-long vacuum chamber for a beamline component test facility (BCTF). The chamber will be equipped with large access flanges for insertion of a variety of test components, such as white-beam photon shutter blades, white-beam masks, or whitebeam slits. Numerous smaller flanges will be used for thermocouple, electrical and water-cooling feedthroughs, infra-red camera and visible light viewports and actuator ports to vary the angle of incidence on the component under test.

In order to test high-heat-load components, the BCTF chamber will be vented and the component will be mounted on an actuation stage. At this time, a fixed mask and photon stop will be mounted and locked in position on the downstream wall of the BCTF chamber. The photon stop will be designed for a maximum 6-kW total beam power with $400 \mathrm{~W} / \mathrm{mm}^{2}$ heat flux.

\section{Soft X-ray Horizontally Deflecting Mirrors}

To deliver undulator radiation to the soft x-ray branch line (4-ID-C), two horizontal deflection mirrors located at approximately 29.9 meters will be inserted into the beam and will deflect the undulator radiation outboard $5^{\circ}\left(1.25^{\circ}\right.$ incidence angle onto each mirror).

The incidence angle of $1.25^{\circ}$ results from the compromise between reasonably high reflectivity for $0.4-3.5 \mathrm{keV}$ x-rays for several mirror materials and sufficient separation between the two branch lines for placement of optical elements upstream. The mirrors will be coated with $\mathrm{Rh}$, which has a cut-off energy of $2.6 \mathrm{keV}$. Multilayers will be used to reach higher energies.

The maximum total power and power density on the first mirror surface are 28.6 $\mathrm{W}$ and $0.9 \mathrm{~W} / \mathrm{cm}^{2}$, respectively. The equivalent mirror designed for the 2-ID-C branch line has an absorbed power one decade higher and a power density two decades higher. Therefore we do not consider this to be a concern in the sector 4 design.

\section{Hard X-ray Branch Line 4-ID-B}

The hard x-ray branch line is designed for polarization manipulation in the $3-100 \mathrm{keV}$ $\mathrm{X}$-ray energy range. The primary goal of this beamline is to use the linearly polarized beam from undulator $\mathrm{A}$ and convert it to circularly polarized beam by using phase retarding crystal optics. Depending on the energy range, different schemes will be used (Lang et al., 1996). In the energy range up to $20 \mathrm{keV}$, a DCM will be used in conjunction with either perfect diamond or silicon phase retarders. In the energy range above $20 \mathrm{keV}$, a germanium phase retarder will be used. A mirror following the DCM will focus radiation in the vertical direction.

\section{Double-Crystal Monochromator (DCM)}

The DCM is the first optical component that is exposed to high-heat-load conditions. Liquid nitrogen cooling of crystals will be implemented, as in the 1-ID beamline. 


\section{Focusing Mirror}

The function of this mirror is to vertically focus radiation into the end station. Since the mirror is placed after the monochromator, substrate cooling is not required. The shape of the mirror is cylindrical, with a radius that can be varied in order to achieve maximum flexibility in focusing.

\section{4-3.5 keV Soft X-ray Spectroscopy Branch Line 4-ID-C}

The 4-ID-C branch line will be dedicated to spectroscopy and instrument development in the intermediate energy range from 0.4 to $3.5 \mathrm{keV}$. The principal aim of this beamline is to preserve the undulator source polarization. It will also maximize flux throughput at relatively high resolving powers, of the order of several thousand. The source polarization will be preserved by using grazing angles of incidence. The entire branch line upstream and including the Y5 vertical focusing mirror assembly will be transposed directly, as is, from branch line 2-ID-C. The only re-engineering involved for this branch line will be the Y4 double mirror assembly. Also a new vertical mirror will be required because of the modified source-to-mirror distance, however the mechanical assembly from the existing sector 2 beamline will be used.

At the end station, a flexible UHV experimental chamber will be used. Currently a two-tier UHV experimental chamber is under testing. This will be used for thin film and multilayer, as well as gas phase, studies. The monochromatic beam will enter the bottom analysis chamber, which is equipped with a Perkin Elmer hemispherical analyzer with a mean radius of $140 \mathrm{~mm}$, an acceptance angle of $\pm 20^{\circ}$ and multichannel electron detector, and an electron gun with $1000-\AA$ beam diameter. The top preparation chamber will be equipped with a reverse-view LEED, an electron gun and hemispherical analyzer for Auger analysis, two thin-film deposition sources, a quartz-crystal thin-film deposition monitor, and an ion sputter gun. Thin-film samples prepared in the upper chamber will be transported into the lower analysis chamber by a precision 600-mm-travel linear manipulator that also has liquid nitrogen and resistive heating capabilities. An electron spin detector will be added to one of the hemispherical analyzers in the near future. In collaboration with the adjacent imaging/coherence beamlines 2-ID$\mathrm{B}$ and 2-ID-E, we also plan to implement a zone-plate-based photoelectron microscope with a spatial resolution of approximately 1 micron.

A photoelectron conversion microscope with a spatial resolution of less than $20 \mathrm{~nm}$ is also being considered. To achieve these levels of stability, an environmental enclosure to minimize acoustic vibrations will be required.

In May of 1998, the MOU for sector 4 was signed and shortly thereafter construction commenced. The first beam in the FOE is planned at the beginning of 1999 . The sector 4 construction and commissioning schedule is presented in Table 5.7. 
Table 5.7 Sector 4 construction and commissioning schedule.

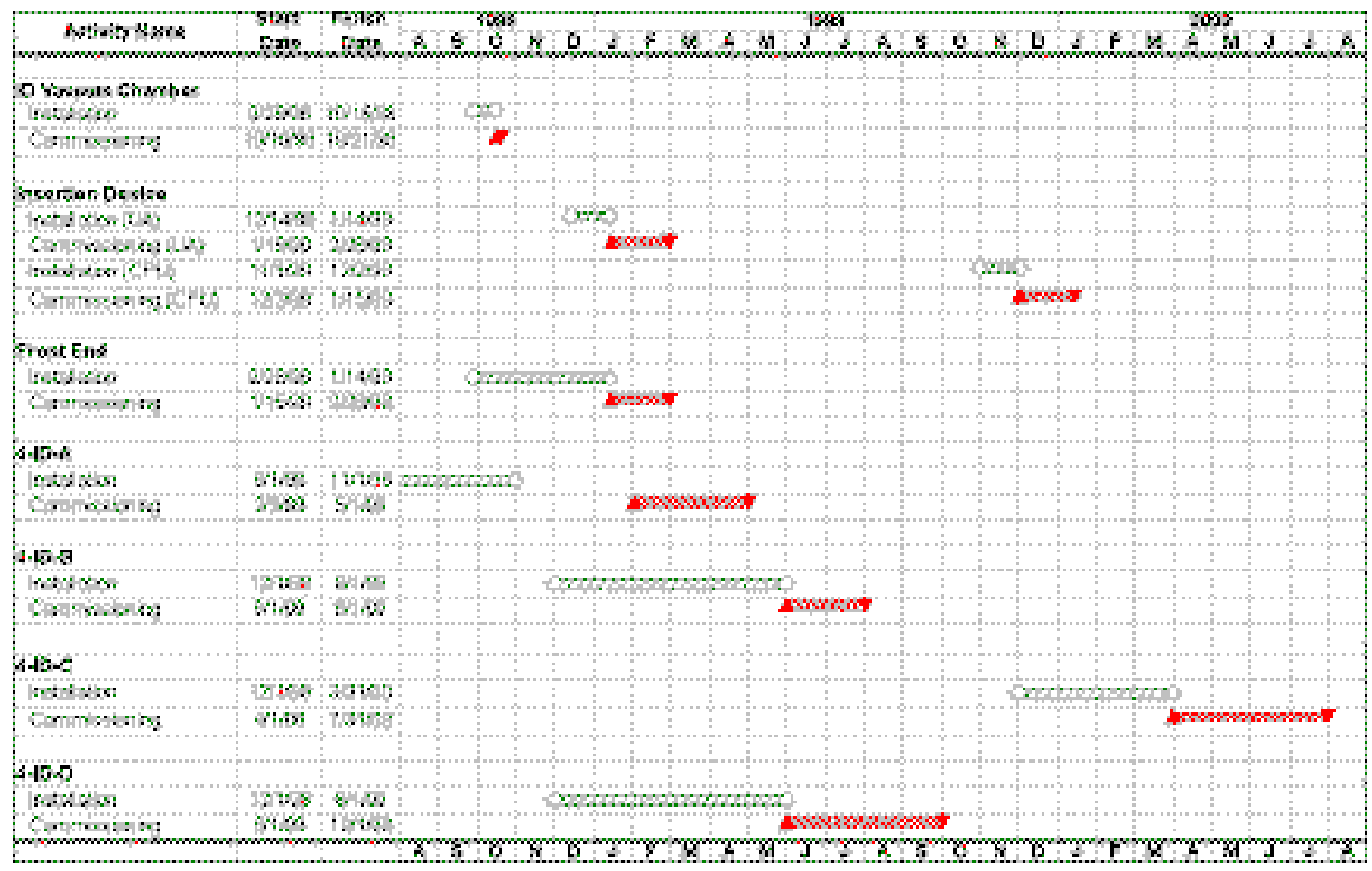




\subsection{References}

Burkel, E., et al. (1989) SPIE Proc. Vol. 1140, 426.

Cai, Z., R. J. Dejus, P. D. Hartog, Y. Feng, E. Gluskin, D. Haeffner, P. Ilinski, B. Lai, D. Legnini, E. R. Moog, S. Shastri, E. Trakhtenberg, I. Vasserman, and W. Yun (1996) Rev. Sci. Instrum. 67 (9) CD ROM.

Camley, R. E. (1989) Phys. Rev. B 39, 12316.

Chumakov, A., J. Metge, A. Q. R. Baron, H. Grunsteudel, H.F. Grunsteudel, R. Ruffer, and T. Ishikawa, et al. (1996a) Nucl. Instr. Meth. A 383, 642.
Chumakov, A., A.Q. R. Baron, R. Ruffer, H. Grunsteudel, H. F. Grunsteudel, and A. Meyer (1996b) Phys. Rev. Lett. 76, 4258.

Colella, R. (1998) N-beam program (Purdue University) available from J. Sutter, Argonne National Laboratory, e-mail: sutter@aps.anl.gov.

Decker, G. (1998) Argonne National Laboratory, unpublished information.

Dejus, R. J., B. Lai, L. Moog, E. Gluskin (1994) Undulator A Characteristics and Specifications: Enhanced Capabilities, Argonne National Laboratory Report ANL/APS/TB-17.

Dorner, B., E. Burkel, and J. Peisl (1986) Nucl. Instr. Methods A 246, 450. 
Fultz, B., C. C. Ahn, E. E. Alp, W. Sturhahn, T. Toellner (1997) Phys. Rev. Lett 79, 937.

Gluskin, E., D. Frashon, P. M. Ivanov, J. Maines, E. A. Medvedko, E. Trakhtenberg, L. R. Turner, I. Vasserman, G. I. Erg, Y. A. Evtushenko, N. G. Gavrilov, G. N. Kulipanov, A. S. Medvedko, S. P. Petrov, V. M. Popik, N. A. Vinokurov, A. Friedman, S. Krinsky, G. Rakowsky, and O. Singh (1995) The Elliptical Multipole Wiggler Project, in Proceedings of the 1995 Particle Accelerator Conference, Dallas, Texas, 1426-1428.

Hahn, U., H. Schulte-Schrepping, K. Balewski, J. R. Schneider, P. Ilinski, B. Lai, W. Yun, D. Legnini, and E. Gluskin (1997) J. Synchrotron Rad. 4, 1-5.

Heidenhain GmbH, ROD 800 catalogue (1996), str. 5, D-83301 Traunreut, Deutschland.

Hewlett-Packard Co., HP-5527B catalogue (1996), S. Manhatttan Ave., Fullerton, CA 92631, U.S.A.

Igor Pro, WaveMetrics, P.O. Box 2088, Lake Oswego, OR 97035, U.S.A.

Ilinski, P., W. Yun, B. Lai, E. Gluskin, and Z. Cai (1995) Rev. Sci. Instrum. 66, 19071909.

Ilinski, P., R. J. Dejus, E. Gluskin, T. I. Morrison (1996) Some Practical Aspects of Undulator Radiation Properties, Optics for High-Brightness Synchrotron Radiation Beamlines II, SPIE Proc. Vol. 2856, 16-25.
Ilinski, P., C. T. Venkataraman, J. C. Lang, and G. Srajer (1997) Characterization of the Elliptical Multipole Wiggler at the Advanced Photon Source, Synchrotron Radiation Instrumention: Tenth U.S. National Conference, ed. E. Fontes (American Institute of Physics) pp. 49-54

Kohzu Seiki Co., Ltd., catalogue (1996), 227-37 Mishuku, Setagaya-ku, Tokyo 154, Japan.

Kushnir, V. I., J. P. Quintana, and P. Georgopoulus (1993) Nucl. Instrum. Meth. A328, 558.

Lang, J. C., G. Srajer, and R. J. Dejus (1996) Rev. Sci. Instrum. 67, 62-67.

LePage, J. G., and R. E. Camley (1990) Phys. Rev. Lett. 65, 1152-1155.

Levine, Z. H., A. R. Kalokin, S. P. Frigo, I. McNulty, and M. Kuhn (1998) Argonne National Laboratory, unpublished information.

Marasinghe, G. K., M. Karabulut, C. S. Ray, D. E. Day, P. G. Allen, J. J. Bucher, D. K. Shuh, Y. Badyal, M . L. Saboungi, M. Grimsditch, S. D. Shastri, and D. R. Haeffner (1998) Argonne National Laboratory, unpublished information.

McLaughlin, J. C., S. L. Tagg, J. W. Zwanziger, D. R. Haeffner, and S. D. Shastri (1998) Argonne National Laboratory, unpublished information. 
Montano, P. A., G. S. Knapp, G. Jennings, E. Gluskin, E. Trakhtenberg, I. B. Vasserman, P. M. Ivanov, D. Frachon, E. R. Moog, L. R. Turner, G. K. Shenoy, M. J. Bedzyk, M. Ramanathan, M. A. Beno, and P. L. Cowan (1995) Rev. Sci. Instrum. 66, 1839-1841.

Mooney, T. (1990) Program "DuMond" based on linearized Bragg equation as given by T. J. Davis (1990) J. of X-ray Science \& Tech. 2, 180.

Optodyne Inc., catalogue (1996), 1180 Mahalo Place, Compton, CA 90220, U.S.A.

Rogers, C.S., D. M. Mills, W.-K. Lee, P. B. Fernandez, and T. Graber (1996) Proc. SPIE Vol. 2855, 170-179.

Schwoerer-Böhning, M., P. Abbamonte, A. Macrander, and V. Kushnir (1997) SPIE Proc. Vol. 3151, 282.

Schwoerer-Böhning, M., A. Macrander, and D. A. Arms (1998) Phys. Rev. Lett. 80, 5572-5575.

Seto, M., Y. Yoda, S. Kikuta, X,. W. Zhang, M. Ando (1995) Phys. Rev. Lett. 74, 3828.

Shu D. (1997) Patent applied for, ANL-IN97-004.

Smither, R. K., A. M. Khounsary, and S. Xu (1997) Proc. SPIE Vol. 3151, 150-163.

Snigirev, A., V. Kohn, I. Snigireva, and B. Lengeler (1996) Nature 384, 49.

Sturhahn, W., T. S. Toellner, E. E. Alp, X. W. Zhang, M. Ando, Y. Yoda, S. Kikuta, M.
Seto, C. W. Kimball, and B. Dabrowski (1995) Phys. Rev. Lett. 74, 3832.

Toellner, T. (1996) Ph.D. Thesis, Northwestern University.

Toellner, T. (1998) Argonne National Laboratory), e-mail: toellner @aps.anl.gov.

Toellner, T., M. Y. Hu, W. Sturhahn, K. Quast, and E. E. Alp (1997) Appl. Phys. Lett. 71, 2112-2114.

Verbeni, R., F. Sette, M. Krisch, U. Bergman, B. Gorges, C. Halcoussis, K. Martel, C. Masciovecchio, J. F. Ribois, G. Ruocco, and H. Sinn (1996) J. Sync. Rad. 3, 426 .

Wang, C., et al. (1987) Laser \& Optronics p. 69.

Wang, J., A. K. Sood, P. V. Satyam, Y. Feng, X.-Z. Wu, Z. Cai, W. Yun, and S. K. Sinha (1998) X-ray Fluorescence Correlation Spectroscopy: A Method for Studying Particle Dynamics in Condensed Matter, Phys. Rev. Lett. 80, 1110-1113

Yamamoto, S., H. Kawata, H. Kitamura, M. Ando, N. Saki, and N. Shiotani (1989) Phys. Rev. Lett. 62, 2672.

Zygo Co., ZMI-1000 catalogue (1996), Laurel Brook Road, Middlefield, CN 06455, U.S.A. 


\subsection{Summary of Major Plans for the Future}

A summary of XFD future plans is provided below, which includes both short-term initiatives and long-term strategic initiatives:

- Funds have been requested from the DOE to improve the performance of various components of major systems, such as insertion devices, front ends, PSS, EPS, etc., that are under XFD responsibility. Shortterm needs will be supported to maintain the reliability of operations in order to deliver maximum beam time to the users. These tasks will receive the highest priority in XFD operations workload. The improvements will be performed using funds made available under the Accelerator Improvement Program.

- During FY 1998, the APS has set aside funds to design and build a liquid nitrogen distribution system on the experiment hall floor. The system will deliver liquid nitrogen to all the first optics enclosures on CAT beamlines to cool the optics. The system will be built during FY 1999.

- Effort and resources will be made available during FY 1999 to support the "top-up" mode at the APS. Implementation requires not only a close evaluation of the impact of the mode on user experiments but also provision for developing required systems to mitigate any difficulties encountered by the users in performing their experiments.
- $\quad$ SRI-CAT will be developing sector 4 for the use of polarized x-rays. The effort to deliver two independent $\mathrm{x}$-ray beams from two undulators located on the same straight section to two independent beamlines poses new technical challenges. These will be addressed during FY 1999. The two radiation sources (undulator A and the CPU in a "dog-leg" configuration on straight-section 4 of the storage-ring), beamline front end, and the beamlines are planned for commissioning in early FY 2000. In the future, this type of dog-leg configuration can add an extra beamline in any sector of the APS by the installation of two undulator sources canted in the horizontal plane on the same straight section.

- The beamline, front end, and undulator for COM-CAT are funded by the State of Illinois. XFD staff are involved in completing the construction of this beamline during FY 1999.

- The APS Phase-2, which was described in the last XFD Progress Report 1996-97, has been submitted to the DOE. This Phase-2 initiative, in which the remaining sectors of the APS will be fully developed, is consistent with the recommendations of the BESAC Panel on DOE Synchrotron Sources and Science, November 1997 (chaired by R. J. Birgeneau and Z.-X. Shen). According to the plans included in the DOE Basic Energy Sciences (BES) Facilities Roadmap (July 1998, Fig. 6.1), this new initiative begins in FY 2000 and extends 


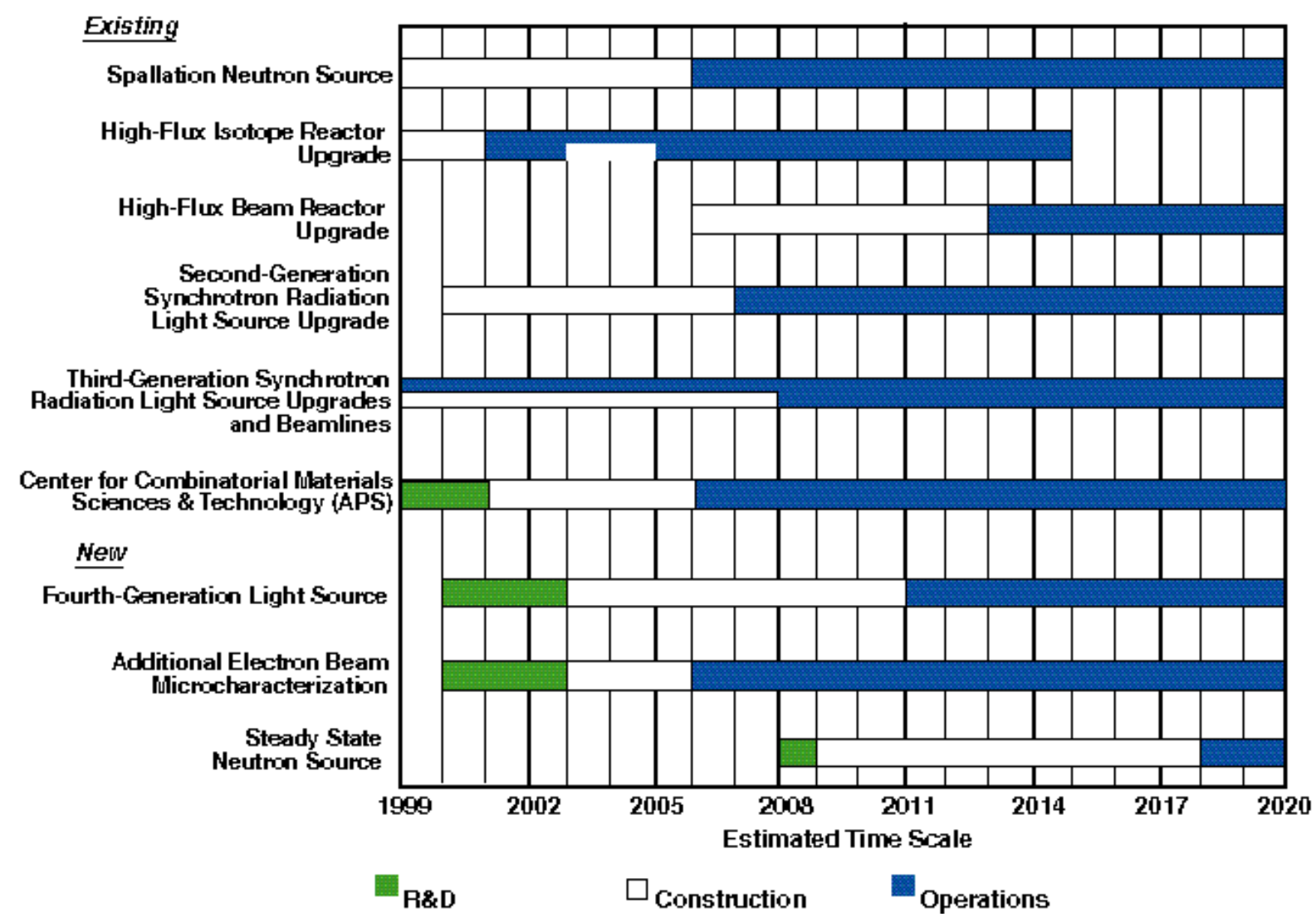

Fig. 6.1 DOE BES Facilities Roadmap

through FY 2008. A more detailed description of the proposal is provided in section 6.2.

- The fourth-generation light source concept has been a major area of R\&D both in ASD and XFD in support of a laboratory initiative. Much effort has gone into the plan for an APS linac-based FEL in order to demonstrate the self-amplified spontaneous emission (SASE) concept in the UV wavelength range. XFD has the major responsibility to develop the undulators and associated particle and photon diagnostics required for the success of the project. Considerable progress in this initiative has been made. Major effort during the past years has gone into the development of a simulation code for the FEL, as well as defining the lattice and tolerances of various components, layout of the FEL undulator line, characteristics of the undulators required for FEL contrasting them with the needs of the APS, and the proposed optical diagnostics to monitor the performance of the FEL. These tasks (supported by LDRD funds) are described in detail in section 6.3. The current plans call for a demonstration of the SASE concept in the visible wavelength range during the summer of 1999 and in the UV range during the fall of 1999. The DOE BES 
Facilities Roadmap (Fig. 6.1) includes a fourth-generation user facility with $R \& D$ funds for the facility starting in FY 2000.

- A major new initiative has been planned to develop a Center for Combinatorial Materials Science and Technology that will use beamlines in a sector at the APS. This proposal will involve staff from both XFD and the Materials Science Division (MSD) at ANL. The initial plan for this center (see section 6.4) was submitted to the DOE and has been well received; DOE has included this in the DOE BES Facilities Roadmap (Fig. 6.1). The center is expected to develop massively parallel techniques for complex materials synthesis using combinatorial methods and massively parallel physical characterization of the materials using microsensors developed using both deep-etch $\mathrm{x}$-ray lithographic techniques and $\mathrm{x}$-ray microprobes.

- Another major new laboratory initiative that addresses structural genomics is called the "Illuminator Project" and involves staff from XFD and the Center for Mechanistic Biology and Biotechnology (CMB) at Argonne. In understanding the "blue-print" of life, the knowledge of DNA sequence coding for thousands of proteins is a prerequisite. While the genome project has made considerable progress towards this goal, the information is insufficient for full understanding of human and other living systems. The knowledge of 3-D protein structures and their folding patterns is an essential next step to make greater strides in this field. In this quest, there are two major bottlenecks in which XFD staff plan to contribute: (a) production of thousands of purified crystals for structural analysis, and (b) measurement of these structures using an APS beamline. Neither can be performed in a time efficient way with existing capabilities. Both these bottlenecks can be overcome through the development of robotics capabilities for crystal growth and operation of "smart" beamlines. In anticipation of funding support for the Illuminator Project, the Laboratory is providing LDRD funds starting in FY 1999 to $\mathrm{CMB}$ and XFD to jointly pursue these tasks (see section 6.5). The benefit of this $R \& D$ is expected to influence all disciplines represented by the APS user community in performing research in a more efficient fashion.

Strategic planning has been performed by XFD to develop new initiatives included in the above list to meet future APS goals. This planning has resulted in four major new initiatives: 1) a Phase-2 plan to develop the remaining beamlines at the APS, 2) a SASE FEL, 3) a proposed Center for Combinatorial Material Science and Technology, and 4) the Illuminator Project. The DOE BES Roadmap, shown in Fig. 6.1, includes three of the above initiatives. In the following sections, brief technical descriptions of these four initiatives are provided. 


\subsection{Phase-2 Initiative}

\subsubsection{Background}

In the completed phase (Phase-1) of the APS Project, 40 of the 68 possible x-ray sources have been readied for use. Within the scope of Phase- 1 construction, IDs and beamline FEs were built and installed by XFD so that the users could build 40 beamlines on the experiment hall floor to perform research. The $40 \mathrm{x}$-ray sources to be completed in Phase- 1 have already been committed to their full use through this century by the scientific and technological users. Two more sectors have recently been awarded (SRICAT sector 4 and COM-CAT sector 32), which leaves 12 more sectors available for the development of Phase-2 beamlines. Demand for beamlines spanning a wide variety of scientific disciplines continues to increase. Many of the new users are also proposing technique-specific beamlines to be developed at the APS to support national communities of users. The concept of technique-specific beamlines is also endorsed by the Birgeneau-Shen committee in their report. These new beamline facilities will be targeted for the most important science/technology goals of the U.S. research community and will be available on a peer-review or proprietary basis to all researchers from universities, federal laboratories, and industry.

In this APS Phase-2 Initiative, 2.5-m-long and 5-m-long ID x-ray sources will be built on 12 straight sections of the APS storage ring, and an additional $12 \mathrm{BM}$ sources will also be put in use. The beamline FEs for these 24 x-ray sources will be built to contain and safeguard access to these bright $\mathrm{x}$-ray beams. In addition, funds will be provided to build an additional six state-ofthe-art technique-specific beamlines to meet scientific and technological research demands well into the next century. These include, for example, the demands of the biotechnology, medical imaging, environmental science, microprobe, and high-energy x-ray scattering communities.

The APS Phase-2 Initiative also proposes to build two laboratory-office modules for the users. These modules are similar to the six included in the Phase-1 construction project. In addition, a specialized laboratory will be added to meet specific scientific goals of the users performing research in the areas of combinatorial materials research (see section 6.4) and biotechnology (see section 6.5).

\subsubsection{Beamline Plan in the APS Phase-2 Initiative}

In the APS Phase-2 Initiative, the plan is to prepare an additional 12 sectors (or 24 beamlines) of the APS. There will be 12 straight sections of the APS storage ring that can accommodate IDs. It is proposed that the IDs in six of these straight sections will be undulators similar to those built in the current phase. Such sectors are referred to as Type A sectors. On the remaining six straight sections of the storage ring, IDs to meet the specific needs of unique scientific and technological research communities will be built. These technique-specific sectors are referred to as Type B sectors and will also include the beamlines to be constructed by the APS staff. Details of the current user status are given in Table 6.1.

In this new initiative, the six Type A sectors will be completed to meet the demand of the 
Table 6.1 Status of Collaborative Access Teams

(CATs) and Scope of Phase-1 and Phase-2

(October 1998)

\begin{tabular}{|l|c|}
\hline APS Sectors $^{\mathrm{a}}$ Operational in Phase-1 & 20 \\
\hline New APS Sectors Assigned (SRI-CAT and COM-CAT) & 2 \\
\hline Sectors Requested by the New Proposals for Phase-2 & 4 \\
\hline Sectors Approved for Phase-2 by the PEB (October 98) & 2 \\
\hline Sectors Waiting for PEB Approval & 2 \\
\hline $\begin{array}{l}\text { Type A Sectors to be Instrumented behind the Shield } \\
\text { Wall in Phase-2 }\end{array}$ & 6 \\
\hline $\begin{array}{l}\text { Type B Sectors and Beamlines to be Instrumented in } \\
\text { Phase-2 }\end{array}$ & 6 \\
\hline $\begin{array}{l}\text { Technique-Specific Beamlines on Type-B Sectors in } \\
\text { Phase-2 }\end{array}$ & 6 \\
\hline Laboratory/Office Modules Built in Phase-1 & 6 \\
\hline Laboratory/Office Modules in Phase-2 & 2 \\
\hline
\end{tabular}

a) A sector at the APS provides two beamlines: one for the ID source and the other for the BM source.

b) Includes two sectors for strategic initiatives described in sections 6.4 and 6.5 .

CATs approved by the PEB. Simultaneously, attention will be given to the special purpose beamlines that will occupy the six Type-B sectors. The technical subjects for beamlines in these sectors will not be decided at this time. However, ten possible subjects for such beamlines have been identified. As the new initiative matures, a decision will be made on the special purpose beamlines using the best expertise from the scientific and technological user community of the APS and the PEB, as recommended by the Birgeneau-Shen Committee. The list of these beamlines includes the following: structural genomics, medical imaging, very high energy x-ray scattering, subnanosecond temporal resolution studies, coherence and interference techniques, three-dimensional imaging, microcomponent fabrication, archaeology and archaeometallurgy, radiation therapy,
X-ray microprobes (microfluorescence, microimaging and microdiffraction), and combinatorial materials science. Many of the LDRD programs in FY 1998 (see section 1.10) support this initiative.

\subsection{FEL Project}

The recent success of third-generation synchrotron radiation sources around the world laid the groundwork for exploring new levels of brightness for VUV and x-ray sources. In the past few years, there have been a number of scientific and technical workshops on the next generation of synchrotron radiation sources. One important outcome of these meetings is the technically well-supported conclusion that a linac-based self-amplified spontaneousemission free-electron laser (SASE FEL) 
could represent a future fourth-generation synchrotron radiation source.

Several laboratories around the world, including the APS, recently started construction of SASE FELs for the VUV/x-ray wavelength range. The APS SASE FEL would consist of two major parts: the $600-\mathrm{MeV}$ linac equipped with a small-emittance electron gun, and a set of twelve undulators that would generate and finally lase VUV radiation. Initially, operations will be at a lower energy in order to produce light at a visible wavelength. This relaxes requirements for the electron beam, undulator, and diagnostics. After experience is gained at $510 \mathrm{~nm}$, the linac energy will be increased to achieve SASE operation at $120 \mathrm{~nm}$. This entire project is supported by LDRD funds.

XFD is responsible for the design, construction and commissioning of the undulator line of the APS SASE FEL, while ASD is responsible for delivering the electron beam. For the XFD staff, the SASE FEL project is a very natural continuation of the ID development process that has been quite extensive during the entire APS project. As of summer 1998, there are 22 IDs installed in the APS storage ring, as described in section 5.1 of this report. The uniquely equipped ID Magnetic Measurement Facility and innovative methods of magnetic tuning provide a solid base of support for the state-of-the-art performance of APS IDs. Also, x-ray diagnostic techniques developed and implemented at the APS permit independent verification and confirmation of ID performance. All this expertise is essential in beginning development of a new generation of synchrotron radiation sources.
The main difference between the development of IDs for the APS storage ring and for the FEL is the integration process. While IDs for the APS storage ring must be integrated into the existing magnetic lattice of the machine, the FEL IDs themselves are the lattice, and therefore the choice of lattice, in most cases, lies in the hands of the ID developers. With this choice comes the responsibility for justifying the lattice and the ID specifications. In order to accomplish this task, new codes for the simulation of SASE FEL performance have been written and extensively tested, and the results were found to agree with results from previous SASE FEL codes.

Once the new codes were validated, their efficiency and flexibility were used to determine that the magnetic lattice for the APS SASE FEL could be based on a separated functions approach, i.e., the undulators and quadrupole lenses do not need to be combined in the same magnetic element. The calculated FEL output power is not affected significantly by the choice of combined or separated focusing functions in the magnetic lattice. This crucial result brings extremely critical simplifications to the FEL undulator design, in that the welldeveloped and well-proven approach used in the design and construction of the APS IDs can be applied to the SASE FEL. In addition, conventional beam diagnostics can be placed along with the quadrupoles in the breaks between separated IDs.

\subsubsection{FEL Computer Code}

A computer code was developed to investigate important design considerations for the APS FEL. For example, the 
possibility of the horizontal focusing being separate from the undulator was studied, and, more recently, mechanical tolerances have been determined. The code numerically integrates the equation derived by Vinokurov (1996) to determine the electron distribution function in a high-gain FEL. The ability to handle longitudinally inhomogeneous magnetic systems, such as separated undulator segments with quadrupole focusing lenses inserted in the breaks between undulators, was specifically built into the code. For the case of a continuous undulator, verifications against semianalytical expressions and other codes, such as GINGER and TDA3D, have been performed, with very good agreement in all cases.

The results of calculations that looked at the difference between a continuous undulator and separated undulator segments with horizontally focusing quadrupoles between the segments are shown in Fig. 6.2. The power output of the FEL is proportional to the modulus, squared, of the electron bunch peak current density. This quantity is expected to grow exponentially for a perfect continuous undulator, with horizontal focusing provided by shaping of the pole tips. The dotted line in the top panel of Fig. 6.2 is indeed a straight line, as expected for a log plot. The solid line shows the result of the simulation for the planned APS FEL design. The amplification is reduced due to the breaks between undulators, but the reduction of $\ln \left(|\mathrm{J}|^{2}\right)$ is the ratio of the break length to the cell (i.e., undulator plus break) length. Thus, the electron bunch peak current density is not adversely affected by a lattice with separate function elements. No extra undulator magnetic structure length is needed to compensate for loss of gain in the breaks. The lower panel of Fig. 6.2 shows the scaling factor $\mathrm{F}$, which is inversely proportional to the power gain length. The integral of $\mathrm{F}$ is also proportional to $\ln \left(|\mathrm{J}|^{2}\right)$.

The calculations were performed at a beam energy of $220 \mathrm{MeV}$ for a beam emittance of $2.50 \times 10^{-8} \mathrm{~m}$-rad (for both the horizontal and vertical directions), and a beam energy spread (s.d.) of $0.15 \%$. The undulator period length was $3.30 \mathrm{~cm}$, and the total length of one undulator segment was $2.3265 \mathrm{~m}$ (70.5 periods). The break length was fixed at $36.5 \mathrm{~cm}$ with a single horizontally focusing quadrupole (offcentered longitudinally by $8.0 \mathrm{~cm}$, and focal length $1.00 \mathrm{~m}$ ) in each break. The undulator $\mathrm{K}$ values were 3.10. These parameters give a fundamental harmonic in the visible light range $(5168 \AA)$. (This differs slightly from the plan for the APS FEL, which is to adjust the beam energy slightly away from $220 \mathrm{MeV}$ in order to have a fundamental harmonic of $5100 \AA$.) The undulators were perfectly aligned, the incident beam was centered, and matched beam parameters at the entrance were used.

We have also examined beam and mechanical tolerances and were able to determine the full set of necessary specifications. The misalignments of one undulator with respect to adjacent undulators were simulated, and the sensitivity to unmatched beam parameters (the Twiss parameters $\alpha$ and $\beta$ ) at the entrance and to a noncentered incident beam $\left(\mathrm{x}_{\mathrm{o}}, \mathrm{x}_{\mathrm{o}}{ }^{\prime}, \mathrm{y}_{\mathrm{o}}, \mathrm{y}_{\mathrm{o}}{ }^{\prime}\right)$ were checked (for a full report see Dejus and Vasserman, 1998). The calculated tolerances given in Table 6.2 are based on requiring that the power output not change more than approximately $10 \%$ for a given parameter. 
Table 6.2 Acceptable tolerances

\begin{tabular}{|c|c|c|}
\hline Parameter & $\begin{array}{c}\text { with centered } \\
\text { quadrupoles, } \mathrm{f}=2.39 \mathrm{~m}\end{array}$ & $\begin{array}{c}\text { with quads } 8.0 \mathrm{~cm} \\
\text { off-center, } f=1.00 \mathrm{~m}^{\text {a }}\end{array}$ \\
\hline Longitudinal undulator displacement & $1.0 \mathrm{~mm}$ & $1.0 \mathrm{~mm}$ \\
\hline Vertical undulator displacement $b$ & $50 \mu \mathrm{m}$ & $<50 \mu \mathrm{m}$ preferred \\
\hline Horizontal alpha function, $\alpha_{\mathrm{x}}$ & 0.20 & 0.20 \\
\hline Vertical alpha function, $\alpha_{\mathrm{y}}$ & 0.20 & 0.20 \\
\hline Horizontal beta function, $\beta_{\mathrm{x}}$ & $0.50 \mathrm{~m}$ & $0.40 \mathrm{~m}$ \\
\hline Vertical beta function, $\beta_{\mathrm{y}}$ & $0.20 \mathrm{~m}$ & $0.15 \mathrm{~m}$ \\
\hline Horizontal incident beam coordinate, $\mathrm{x}_{\mathrm{O}}$ & $200 \mu \mathrm{m}$ & $100 \mu \mathrm{m}$ \\
\hline Vertical incident beam coordinate, $\mathrm{y}_{\mathrm{o}}$ & $50 \mu \mathrm{m}$ & $<50 \mu \mathrm{m}$ preferred \\
\hline Horizontal incident beam angle, $\mathrm{x}_{\mathrm{o}}{ }^{\prime}$ & $100 \mu \mathrm{rad}$ & $50 \mu \mathrm{rad}$ \\
\hline Vertical incident beam angle, $\mathrm{y}_{\mathrm{o}}{ }^{\prime}$ & $50 \mu \mathrm{rad}$ & $<50 \mu$ rad preferred \\
\hline
\end{tabular}

a) These additional simulations were done after Dejus and Vasserman (1998), for other conditions being considered for the APS FEL.

b) Horizontal displacement much more relaxed: use $1.0 \mathrm{~mm}$.

\subsubsection{The FEL line}

The simulations confirmed that a separatedundulator approach was reasonable. This approach gives greater flexibility in the diagnostics and allows us to build on our existing undulator expertise. The FEL line will consist of a series of identical cells, where each cell includes an undulator, a diagnostics section, and a quadrupole singlet. The quadrupole and diagnostics section will be located in the gap between consecutive undulators. A total of 12 cells is planned.

The length of the gap between successive undulators must be carefully chosen so as to maintain the proper phasing between undulators. The length of the section also depends on the strength of the undulator magnetic field. The undulators will be adjusted to a $\mathrm{K}$ of 3.1 , corresponding to an effective magnetic field of $10.061 \mathrm{kG}$. The length of the break between undulators will be $36.5 \mathrm{~cm}$ from the last full-field pole (the next-to-last pole) of one undulator to the first full-field pole (the second pole) of the next undulator. This break can accommodate the quadrupole and the optical diagnostics. When the length of the full-field region of the undulator is included, the cell length becomes $269.15 \mathrm{~cm}$.

The undulator magnetic field itself provides vertical focusing of the particle beam. Horizontal focusing is provided in the space between undulators by the quadrupole magnet. The FEL simulation codes were used to evaluate a variety of different lattices. Configurations with a single quadrupole, a doublet, or a triplet placed in the break between undulators were considered. The singlet was found to give the best particle beam bunching. The codes were also used to optimize the strength of the quadrupole so as to maintain the best bunching within the particle beam. Once the strength of the quadrupole was chosen, the 

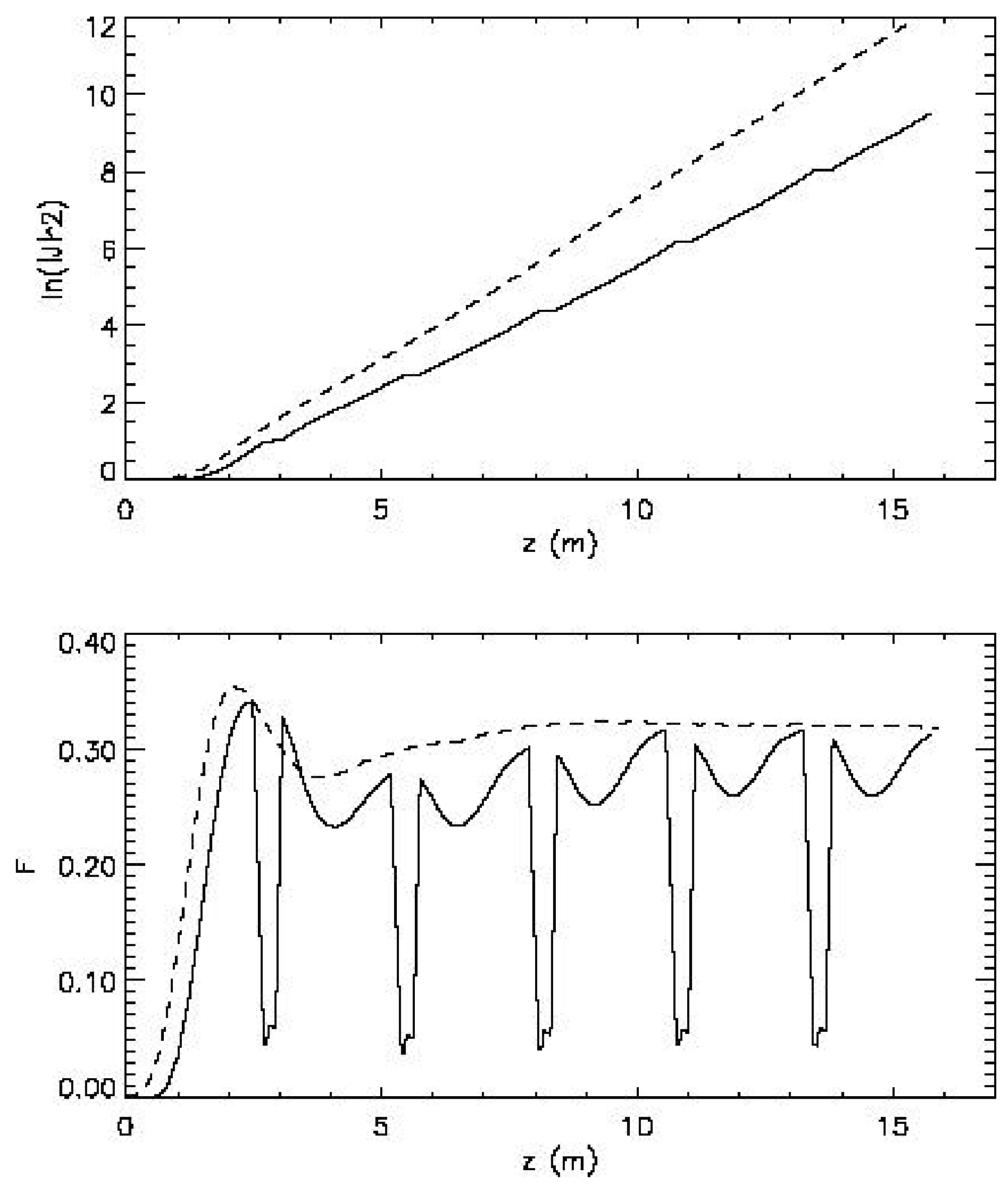

Fig. 6.2 (top) Natural logarithm of the modulus squared of the electron bunch peak current density for a continuous undulator (dashed) and the APS FEL design with break sections (solid). (bottom) The dimensionless scaling factor $F$ for the continuous undulator (dashed) and the APS FEL (solid). 
beta function for an undulator cell could be calculated. The result is shown in Fig. 6.3.

The first quadrupole magnet has been assembled and characterized. In addition to providing the quadrupole field to focus the particle beam horizontally, this magnet has windings to allow it to serve as a dipole correction magnet, steering the beam vertically and horizontally. Much attention has been paid to precision in the fabrication of the magnet components, in order to keep the geometric and magnetic axes of the magnet coincident. The aperture of the magnet will be $12 \mathrm{~mm}$.

\subsubsection{Characteristics of the FEL Undulator}

The period length of the undulators for the FEL is $33 \mathrm{~mm}$. Simulations were carried out of the expected gain using period lengths as short as $27 \mathrm{~mm}$, but the results show very little sensitivity to changes in the period. Therefore, the decision was made to proceed with the $33-\mathrm{mm}$-period undulator that is

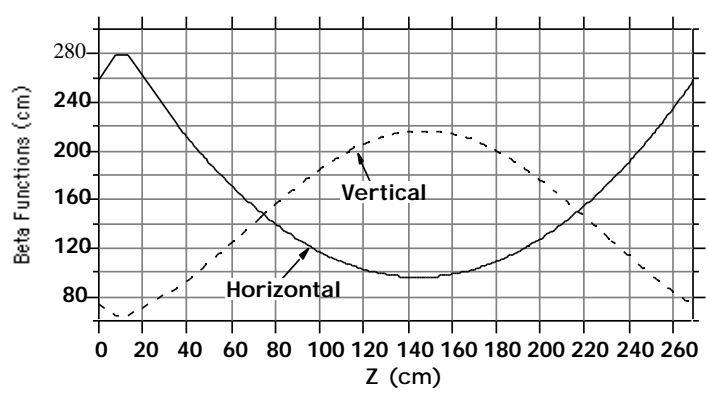

Fig. 6.3 The beta function for a cell of the FEL lattice. The cell consists of: a 7.785-cm break, followed by a 5-cm quadrupole, then a 22.665-cm break for optical diagnostics, and finally a 233.7-cm undulator (not including the end poles). already well-understood at APS, and the design for the FEL magnetic structure will be identical to that of the standard APS undulator A. A photo of one of these magnetic structures, mounted as it will be in the FEL tunnel, is shown in Fig. 6.4. The undulator $\mathrm{A}$ and FEL magnetic structures are mechanically identical, and the magnetic structures for the FEL will be built by STI Optronics.

Some of the magnetic tuning requirements for FEL undulators are more demanding than those for a storage ring undulator. For an FEL, it is critically important that the particle beam path coincide with the axis of the emitted radiation and that the coincidence extend not just over the length of one undulator but through the entire series of undulators. This means that the trajectory of the particle beam must stay straight through the undulator end regions as well as through the full-field regions. This requirement translates into the requirement that the second field integral (averaged over each period) remain less than 3300 G-cm² through the entire length of the undulators, including the end sections. For a beam energy of $220 \mathrm{MeV}$, this would correspond to a trajectory displacement of $45 \mu \mathrm{m}$. (The corresponding requirement for a storage ring undulator is that the second field integral through the full-field region be below $10^{5}$ G- $\mathrm{cm}^{2}$ for all gaps, with no special requirement for the ends.)

Another requirement for the FEL undulators is that the effective magnetic field strengths for each undulator must be nearly identical so that the light produced by one undulator is at the resonant wavelength for the next. Simulations are being carried out with different field strengths assigned to different undulators. An initial guess is to require that the wavelengths from different undulators 


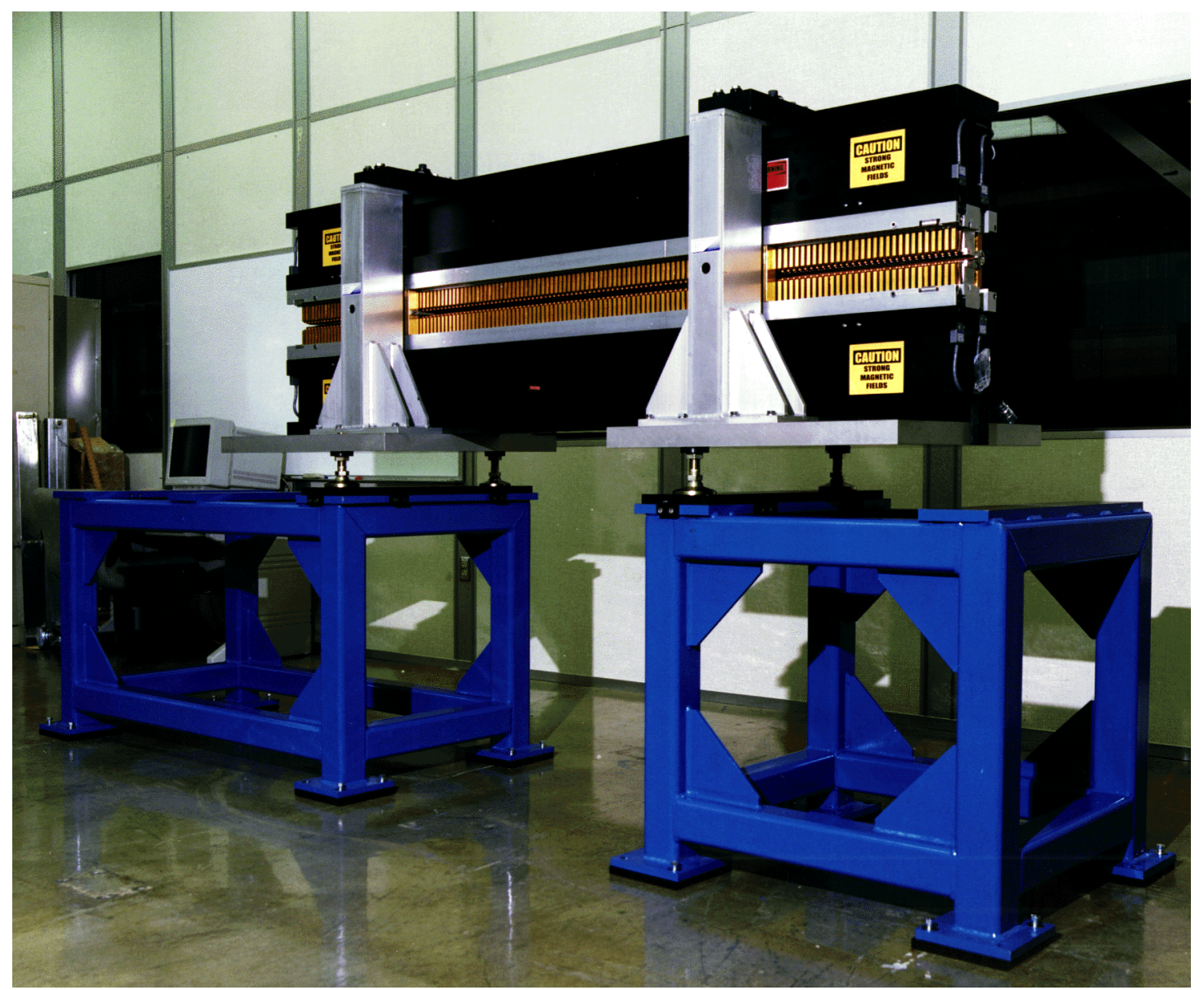

Fig. 6.4 One of the undulators, mounted as it will be in the FEL tunnel.

must be the same to within $5 \%$ of the width of the first harmonic peak from one gain length's worth of undulator. This results in the requirement that the undulator parameters $\mathrm{K}$ be the same to within 1.5 parts in 1000, which translates into the requirement that the effective magnetic fields of the undulators be the same to within $15 \mathrm{G}$. This error in the magnetic field would result if the gap of the undulator were mis-set by $16 \mu \mathrm{m}$. In order to achieve the magnetic field values needed for a $\mathrm{K}$ of 3.1 , the undulator gap will be near $9.3 \mathrm{~mm}$, but the gap of each undulator will be adjusted individually to ensure field strength uniformity between undulators.
Other FEL requirements are less demanding than the corresponding requirements for storage ring undulators. Because the FEL undulators will operate at a single fixed gap, magnetic tuning only needs to be done at that one gap. Also, a small phase error is important for a storage ring undulator to ensure high brightness in higher order harmonics, whereas FEL operation only relies on the first harmonic radiation being bright. Since the brightness of the first harmonic is much less affected by phase errors than the brightness of higher harmonics, the rms phase error requirement is less demanding for an FEL undulator. The criterion used for the FEL is that the first 
harmonic intensity should not decrease by more than $5 \%$ due to phase errors. This leads to the requirement that the rms phase error be less than $10^{\circ}$.

\subsubsection{The Optical Diagnostics}

The diagnostics serve two purposes: 1) to monitor and maintain the alignment between the particle beam and the undulator radiation, and 2) to evaluate the characteristics of the light that is produced by the FEL.

A schematic of the diagnostics section that will be located between the undulators is shown in Fig. 6.5. Since it is critical that the particle beam and the axis of the emitted light beam coincide through the entire series of undulators, three different and complementary monitors of the particle beam position have been included. The capacitive button BPM, or beam position monitor, is the same as the BPMs used at the ends of the insertion device straight sections in the APS storage ring. The relative positions of the buttons are different than in the storage ring; however, because the FEL vacuum chamber has a smaller vertical aperture than the usual storage ring ID vacuum chamber, the buttons will be vertically closer. They will also be closer transversely in order to improve their sensitivity. The wire BPM is an absolute position monitor that consists of two perpendicular sets of four parallel wires. The wires are spaced $0.5 \mathrm{~mm}$ apart and have a diameter of $15 \mu \mathrm{m}$. The current to individual wires is monitored as the particle beam is steered to strike the wires. The beam can be centered vertically and horizontally by determining where it hits the wires on opposite sides of the beam center line and splitting the difference. During normal operation the beam will not strike the wires because the spacing between wires will be a few times the beam size. The third beam position monitor is the YAG scintillator crystal. The optics that will be used to image the YAG crystal will give a 1:1-sized image of the YAG crystal on a CCD camera, so that the $6.5 \times 6.25 \mu \mathrm{m}$ pixel size will be the resolution at the crystal. Therefore, the optical resolution will be comparable to the $10 \mu \mathrm{m}$ resolution reported for the YAG crystal itself (Safranek and Stefan, 1996).

Upstream of the undulators, there will be a chicane for the particle beam. The synchrotron radiation produced at its bends will be monitored as a means of characterizing the particle beam, and it will also provide a place for an alignment laser to be inserted. The alignment laser will be directed down the inside of the vacuum chamber and will be used to define the desired straight-line beam path. Since the alignment laser light will travel through the same optical systems as the FEL light and the light from the YAG crystal, the desired position of the light on the CCD arrays can be defined.

In order to relate the overlap between the particle and emitted light beams inside the undulators to where it is measured between the undulators, it is convenient to require that the particle beam stay in a straight line, with no displacements or deflections, through the ends of the undulators. (The particle beam needs to be straight through the length of the full-field region of the undulators in any case.) Because no position monitors will be located inside the undulators themselves, careful magnetic characterization and tuning of the undulators will ensure the straightness of the trajectory through the ends as well as through the full- 


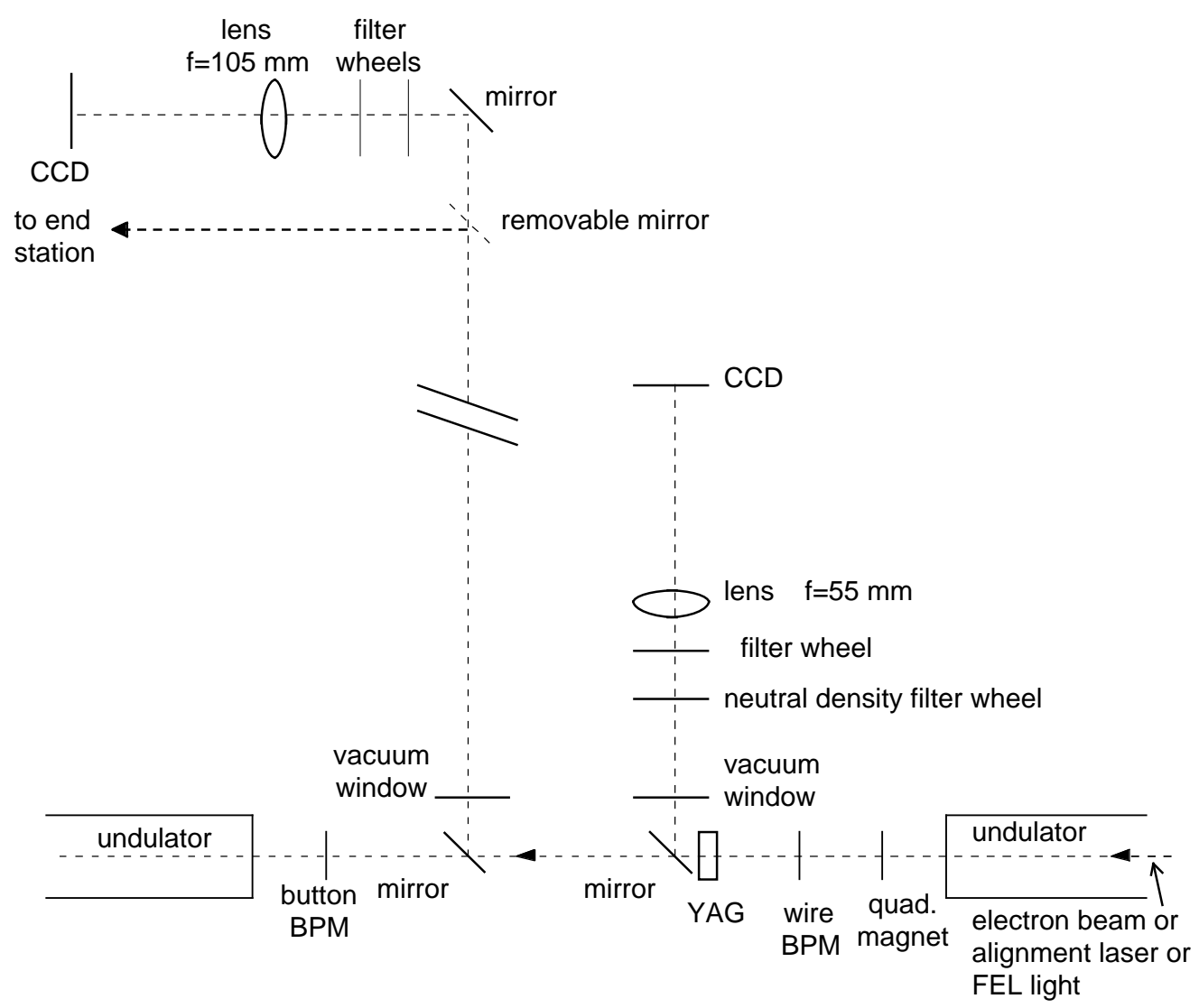

Fig. 6.5 Schematic of the diagnostics section (not to scale)

field regions. The allowed limits for displacement and deflection are a fraction of the size of the particle beam and of the opening angle of the radiation, respectively.

It should be possible to be able to confirm that the trajectory inside the undulator is as expected, despite the absence of position monitors there. The optical systems will provide this ability. The lens and CCD in the upper left of Fig. 6.5 will be used to check the angular deflection inside the undulators. The CCD will be placed at the focal distance from the lens, so that all the light that is incident parallel to a particular angle will be imaged to the same point on the CCD. In this configuration, all position information about the incoming light is lost and the image on the CCD will reflect the distribution in angle of the incoming light. If there is an angular deflection between undulators or a trajectory kick within an undulator, the CCD image will show an apparent displacement.

There are two filter wheels in the upper left of Fig. 6.5. One of them will carry bandpass filters. The other filter wheel will carry a variety of neutral-density filters so that the light levels can be adjusted to suit the CCD.

Another way in which the lens and CCD in the upper left of Fig. 6.5 will be used is with an adjustable distance between the lens and CCD. The CCD will not always be at the 
focal distance from the lens. Instead, the focus of the optical system will be adjustable so it can fall at different distances along the undulator (or undulators). When the optics are used in this way, the positions of the emitted light at different positions along the undulator will be monitored.

For the purposes described thus far, the bandpass filter used will be one that passes the on-axis FEL light. A different bandpass filter, one that passes light that is slightly red-shifted from the on-axis light, can be used instead. The red-shifted, off-axis light that will be passed by this filter will be in the shape of a cone around the axis, and the angle between the cone and the axis will depend on the wavelength passed by the filter. Although the resolution of the optical system is no different when viewing redshifted vs. on-axis light, using the redshifted light to guide adjustments of the relative trajectories through two consecutive undulators may allow more accurate adjustments. The red-shifted light appears as a ring, and two rings may be easier to align than two spots. Also, the width of the annulus of red-shifted light is smaller than the size of the on-axis spot, so the difference is between aligning two sharp rings as opposed to two broader spots.

As shown in Fig. 6.5, a mirror is inserted into the particle (and light) beam path in order to reflect the FEL or alignment laser light into the optics at the upper left of the figure. This mirror will have three positions: one where the mirror is removed from the beam path, one where the mirror completely blocks the beam and reflects all the light, and one with a hole in the center so that there is nothing directly in the path of the particle beam but any light at more than $0.2 \mathrm{~mm}$ from the axis will be reflected into the optical system. Demanding requirements have been placed on the motion of this mirror so that the position of the light on the CCD is repeatable to within a pixel despite the approximately 1-m-long distance between this mirror and the next mirror in the light path. In order to more readily achieve this repeatability, the direction of motion of the mirror between its different positions is parallel to the plane of the mirror face.

Another use for the optics in the upper left of Fig. 6.5 is as a diagnostic for the light produced by the FEL. Each set of these optics will be calibrated for absolute intensity. They will then be used to measure the intensity from each undulator individually, as follows. The mirror in the particle beam path after the first undulator will be positioned so that the $400-\mu \mathrm{m}$ hole allows the particle beam to pass unobstructed. A small fraction of the undulator light will also pass through the hole, but most of it will be reflected into the optics where the absolute intensity of the light from the first undulator will be measured. The small amount of light that passes through the hole is important because it is the light that will interact with the particle beam in the second undulator to induce the bunching needed for lasing. When the light is viewed after the second undulator, the contribution from the first undulator will be a small portion of the total intensity; almost all the intensity will be from the second undulator. If no lasing is occurring in the second undulator, then the absolute intensity seen in the optics after that undulator will be the same as the intensity after the first undulator. This comparison of intensities will be made along the entire line of undulators.

A second diagnostic of the FEL light will be located in an end station, downstream of the 
line of undulators. A Paschen-Runge-type spectrograph will be placed on the lowradiation side of a shielding wall at the downstream end of the undulator line. A schematic of the spectrograph is shown in Fig. 6.6. It will be used for high-resolution spectral measurements near the first harmonic, and, since the goal is to measure the spectral structure in the SASE light, each pulse from the linac will be individually measurable. Light sent to this station will have been picked off after any one of the undulators (including after the last undulator), using the removable mirror shown in the upper left of Fig. 6.5. It will pass through a hole in the shielding wall and be sent to the spectrograph. The slit size will be changeable; for a $20-\mu \mathrm{m}$ slit width the spectral resolving power will be $\lambda \Delta \lambda=$ 13900. After cooling the CCD to reduce the dark current and noise level, the light per incident electron bunch and per pixel from a single undulator with no FEL amplification is expected to be at least 20x higher than the background per pixel. The charge collected in all the pixels in a column will be added together to make the signal-to-noise ratio even more favorable.

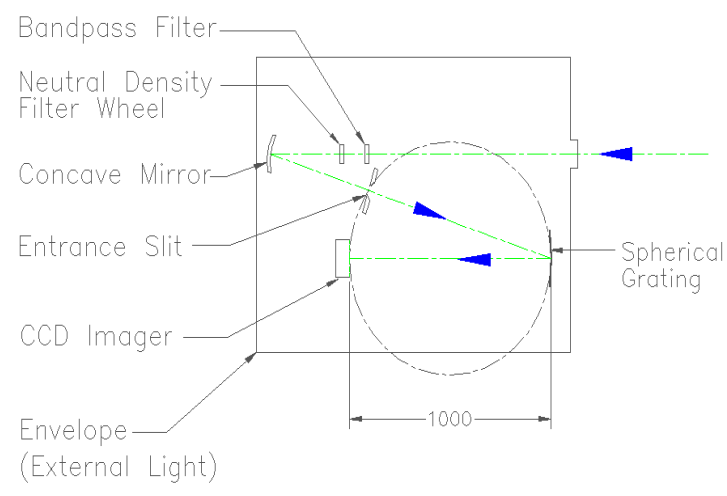

Fig. 6.6 A top view of the Paschen-Rungetype spectrometer that will analyze the light from the SASE FEL.

\subsection{Center for Combinatorial Materials Science and Technology}

In 1997, the National Research Council completed a study on the subject "The Physics of Materials: How Science Improves Our Lives." The complete study will be published later in 1998. It emphasizes the fact that many of the discoveries in materials science during the present century have had a major impact on the technologies of modern times, on our powerful economy and, as a result, on human well-being. Examples include the discovery of transistors, which led to modern silicon technologies, the discovery of fiber optics has led to the communication revolution, the discovery of compound semiconductors has miniaturized communication hardware, the discovery of superconductivity has led to numerous scientific instruments including the MRI, the discovery of liquid crystals has provided the bread-and-butter to the photoconductive display industries, and so on. The materials involved in all these applications are simple and are truly materials of the past. Recently, materials scientist have focused their attention to "complex materials"-materials containing many elements that show complex ternary, quaternary, or higher order phases. They also demonstrate a complex mix of new and rich properties. They exhibit new physics involving strong coupling between electron-, phonon-, and spinorderings.

Their synthesis, discovery, and characterization however cannot be carried out in the traditional methods used in this century involving a trial-and-error approach, which is both inefficient and time consuming when systems are complex. 
Hence one requires a new approach based on systematic and massively parallel synthesis and characterization. The development of new tools to search for complex materials will lead to a revolution in materials science and technology in the next century.

A combinatorial approach to materials synthesis is a new field (Xiang et al., 1995). The method lends itself as an efficient, systematic, and massively parallel process to synthesize an as-yet-unexplored universe of complex materials made up of ternary, quaternary, and higher order materials libraries. An example of producing combinatorial materials is shown in Fig. 6.7. While the technology of producing combinatorial libraries of 100 to 10,000 differing materials is becoming feasible, the limitation in the discovery process for materials with new and unique properties is the ability to measure the physical properties of such libraries quickly and efficiently in a massively parallel process. Thus highthroughput microtechniques must be developed to measure the physical properties of materials libraries if progress is to be made. Figures 6.8-6.10 show examples of a few microtechniques that could be used to measure various properties of combinatorial samples. Having measured

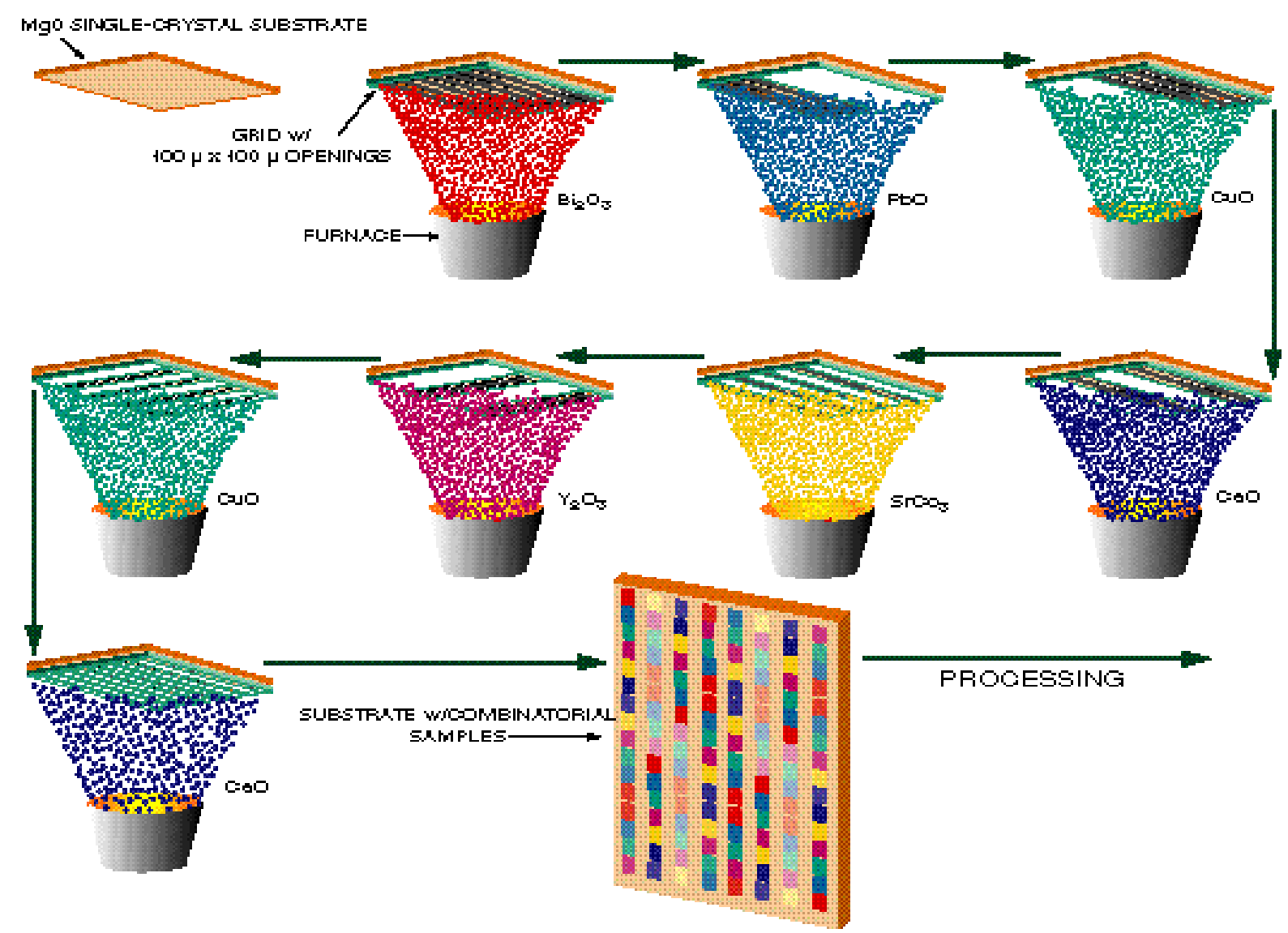

Fig. 6.7 Combinatorial synthesis of pseudo-ternary compounds $(\mathrm{Bi}, \mathrm{Y})-(\mathrm{Sr}, \mathrm{Co})-(\mathrm{Pb}, \mathrm{Cu})-\mathrm{O}$. 


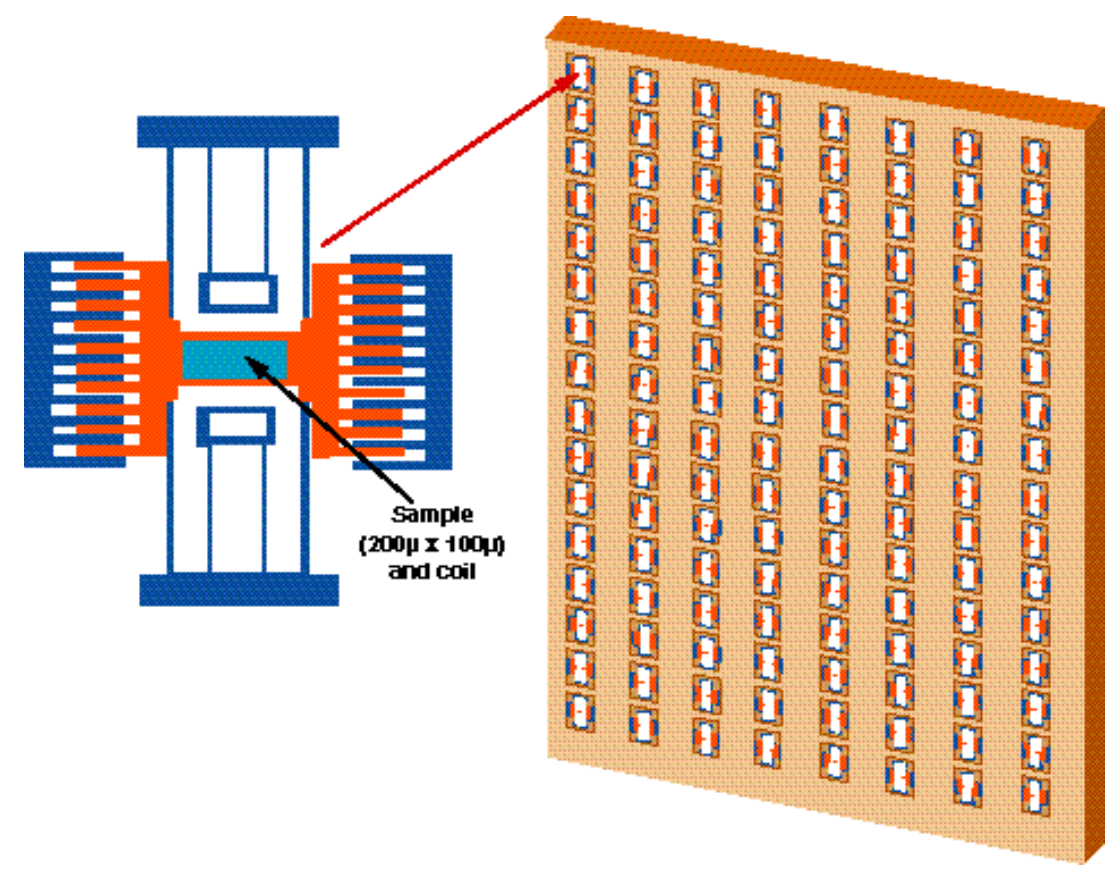

Fig. 6.8 Microsensors for the determination of magnetic properties using the principle of a vibrating coil magnetometer.

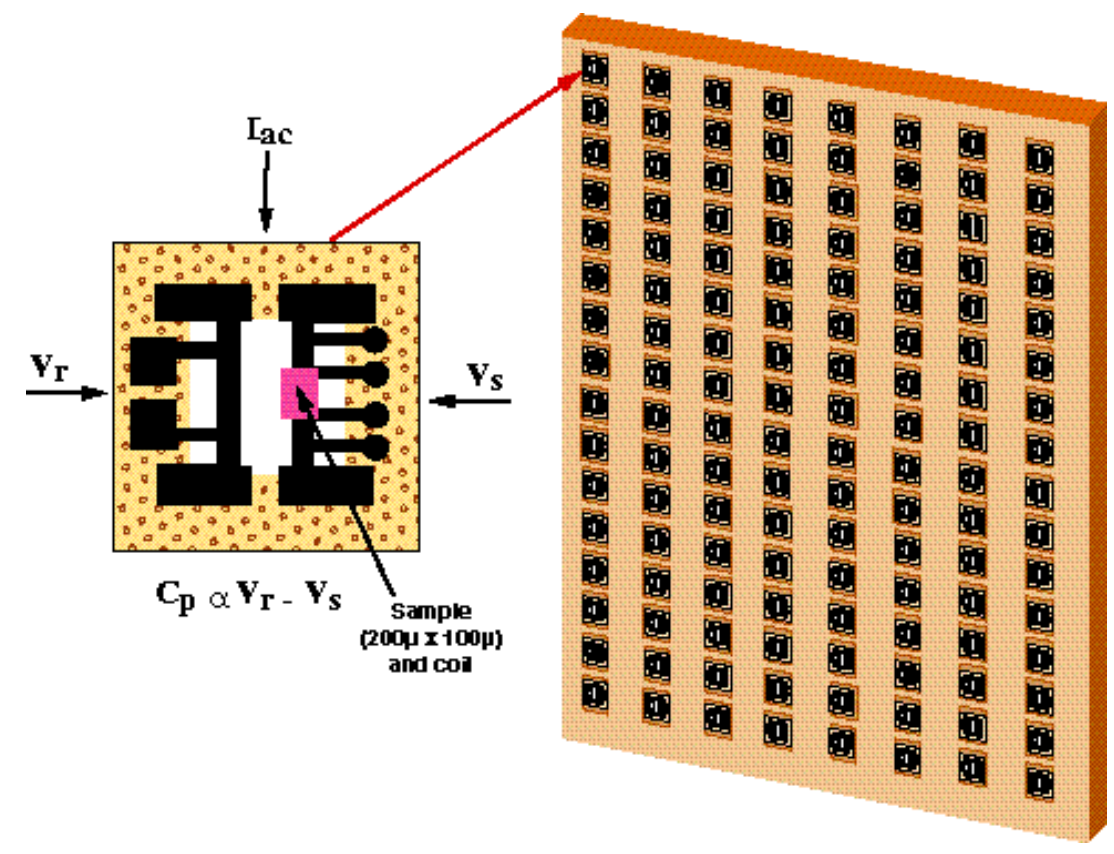

Fig. 6.9 Microsensors for the measurement of heat capacity based on a differential calorimeter. 


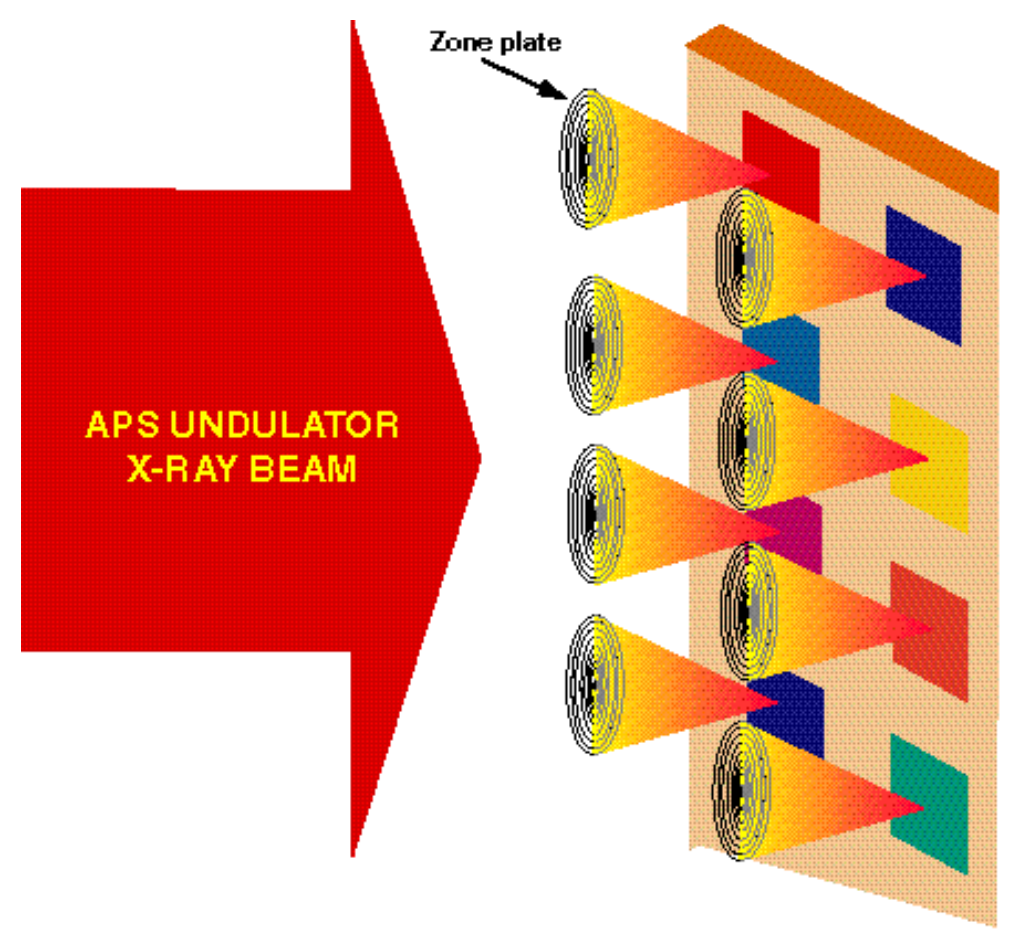

Fig. 6.10 Multiple microbeam materials analysis of combinatorial samples to obtain phase and structural information.

the physical properties, it is then necessary to interpret and understand this vast amount of information. The primary requirement for making progress in this area is to determine the combinatorial possibilities of atomic configurations in a new material in the library. Second, an evaluation of the local structure of materials in the library is also important in interpreting the measured properties of these materials. This process is summarized in Fig. 6.11.

The proposed APS Center for Combinatorial Materials Science and Technology will use the unique capabilities of the APS x-ray beams. The goal of the Center is to develop high-throughput micromeasurement tools and techniques using the high-brightness $\mathrm{x}$-rays from the APS in conjunction with combinatorial processes, which will lead to an efficient and optimized process for the discovery of new materials required for new technologies. The masks for production of both bulk- and nano-phase combinatorial material libraries will be fabricated at the Center using micromachining capabilities now being developed at the APS. The same micromachining fabrication technology will be used to develop microtechniques to measure mechanical, thermal, electrical, magnetic, and optical properties of material libraries with extremely high speed. The final part of physical measurements will involve use of submicron x-ray beams already being produced at the APS to map three-dimensional atomic/molecular configurations in combinatorial material libraries and the local chemical and 


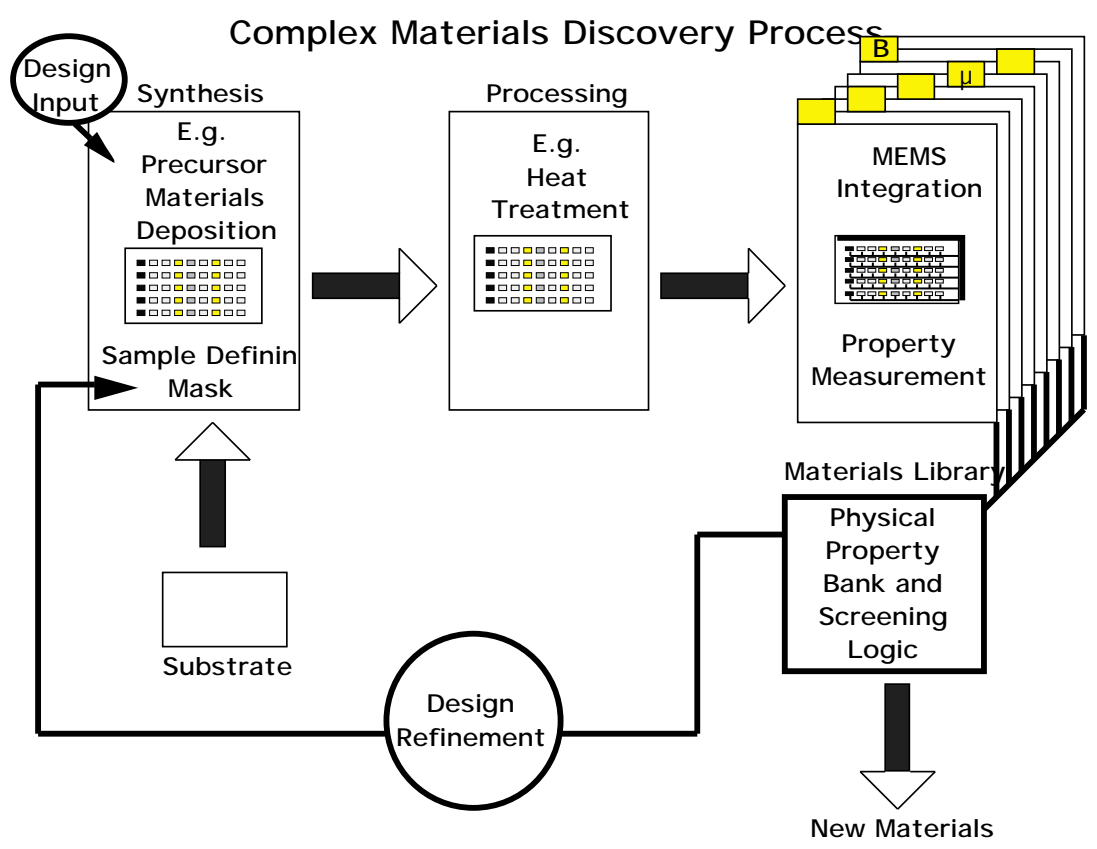

Fig. 6.11 A laboratory on chips.

magnetic structure in these systems. The high brightness of the APS x-ray beams is essential for fabrication of apparatus for the microtechniques required to measure both physical and structural properties. The present plans call for the measurement and understanding of the physical properties of both hard and soft combinatorial material libraries of interest to materials scientists, chemists, and biologists.

A recent National Academy Report concludes that: "Increasingly sophisticated equipment has become necessary for scientific innovation, from electron-beam instruments to giant $x$-ray synchrotrons." Consistent with this statement, the DOE has included a center for combinatorial materials science and technology in the DOE BES Facilities Roadmap (Fig. 6.1).

Our proposal would involve construction of a set of laboratories occupying approximately a 30,000 sq. ft. area adjacent to the APS. These laboratories will be equipped with chemical and physical tools for the production of combinatorial material libraries in various forms, from amorphous materials to single crystals. A specialized laboratory will be dedicated to micromachining technology to support deepx-ray lithography and conventional microfabrication for the development of analytical tools for high-speed microelectromechanical systems (MEMS). An additional laboratory will be dedicated to chemical and biological work. Four beamlines will be dedicated to the Center, one for micromachining, the second for microimaging, the third for microdiffraction, and the fourth for micro-XAS. The synchrotron techniques required to scan libraries with as many as 10,000 materials in a very short period with robotics manipulation of the samples will be developed. The operations of the Center will be the joint responsibility of the APS 
Experimental Facilities Division and the Material Science Division at Argonne National Laboratory.

\subsection{Structural Genomics Project}

A structural genomics initiative is being jointly developed under the leadership of the Center for Mechanistic Biology and Biotechnology (CMB) and XFD. The initiative will focus on the concept of using genomic data as the basis for the selection of proteins for which determination of structure will provide new knowledge of the relationship between amino acid sequence and three-dimensional structure identifying unique folding motifs. It is suggested that approximately 5000 key structural homology groups emerging from the results of Human Genome project represent a viable irreducible set that could eventually provide a virtually complete "almanac" of natural protein structures. Under an optimal scenario where all the required crystals are readily available and the beamline on an APS undulator is capable of uncovering structures without any bottleneck, the structural almanac will conservatively take 5 to 10 years. The reality is far from this optimal situation. The initiative will hence address the two key bottlenecks in accomplishing this task. The known bottlenecks are the following: 1) conventional technologies cannot deliver 1000 crystals of proteins desired per year, and 2) the sample delivery (mounting, aligning and cooling the single crystals) to a beamline, data collection, and structure analysis of 1000 crystals per year cannot be achieved with present day experimental techniques.
The strategy to be used to generate highthroughput production of protein crystals may be thought of as a parallel and iterative production and expression (PIPE) approach. We assume that in order to achieve 5000 crystals, all of which represent new proteins, we must attempt to crystallize 50,000 proteins. The critical step in the crystallization process is growth monitoring. A detailed analysis indicates that over a thousand examinations have to be performed per day using polarized light, which interrogates the reflectance and/or transmittance of the crystallization chamber. The design of an interrogation robot will be the responsibility of XFD.

The other limitation in reaching the target goals of this initiative in structure determination is the current capability of an APS beamline. The target goal is governed by the need to determine the structure of a large number of crystal protein samples. The following additional constraints increase the number of samples. They are (1) short exposures to $\mathrm{x}$-rays in order to reduce the crystal damage from radiation, thus increasing the number of samples of one kind to be studied, and (2) additional data sets to be collected for each structure with different contrast agent (such as $\mathrm{Se}, \mathrm{Br}, \mathrm{Eu}$, $\mathrm{Re}, \mathrm{Os}, \mathrm{Ir}, \mathrm{Pt}, \mathrm{Au}, \mathrm{Hg}, \mathrm{U}$ ) and at least two $\mathrm{x}$-ray energies above and two below the absorption edge for a complete MAD analysis. The bottleneck for collecting data sets on each sample is the constant need to change and realign each single-crystal sample. This task currently is both manpower intensive and time consuming. The principal part of this initiative is to develop robotics technique in order to increase the efficiency of sample delivery to the APS undulator beam. This involves automation of the crystal changing procedure, which includes the following: 
(a) an automatic or semi-automatic mounting tool that will retrieve the crystal from a crystallization droplet to a mounting device, (b) a micron-precision crystal prealignment station outside the experiment station, (c) a micron-precision crystal mounting goniometer, (d) a liquid nitrogen compatible sample transport system that will permit transfer of the pre-aligned sample into the experiment station, (e) a multiplesample mounting system or cartridge that will permit automatic sample change, and (f) new alignment tools and procedures. The goal here is to reduce the frequency of experiment station access and to reduce the time taken to align the sample. The challenge is to maintain the samples at cryogenic temperatures throughout the process from crystal mounting to alignment to the actual exposure to x-rays during data collection. A proposed multiple-sample mounting stage is shown in Fig. 6.12.

This initiative will receive LDRD support starting in FY 1999 for a period of three years. The success of the developments in this initiative can be transferred to many of the APS beamlines to optimally utilize the beam time when ever possible.

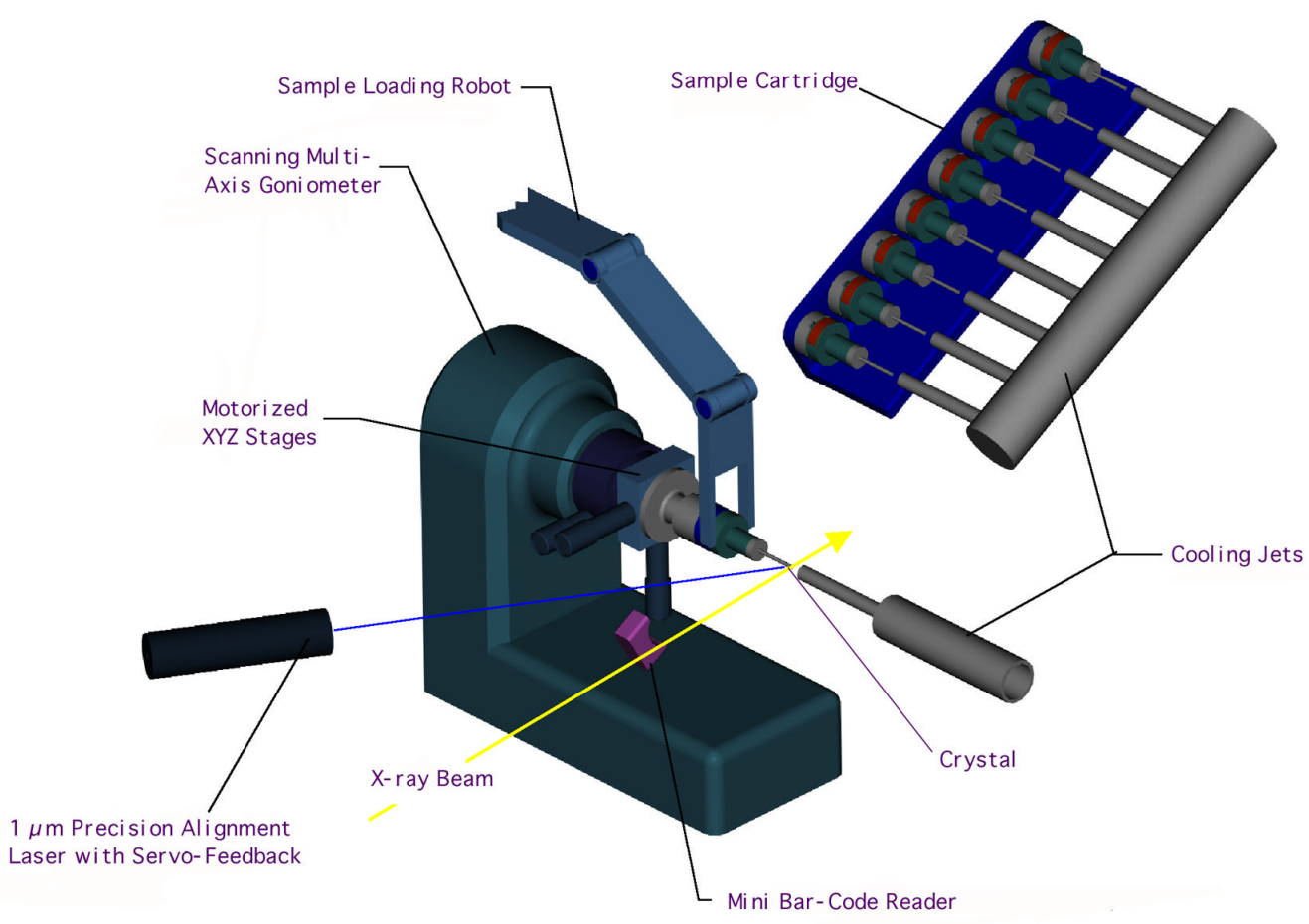

Fig. 6.12 Multiple sample mounting stage. 


\subsection{References}

Dejus, R., and I. Vasserman (1998) Argonne National Laboratory, unpublished results.

Safranek, J., and P.M. Stefan (1996) Proceedings of EPAC '96, the Fifth European Particle Accelerator Conference, p. 1573.

Vinokurov, N. A. (1996) "The Integral Equation for a High Gain FEL," Argonne National Laboratory Report ANL/APS/TB27.

Xiang, X.-D., et al.(1995) Science 268, 1738. 


\section{7-1998 Publications by XFD Staff}

Agamalian, M., J. M. Drake, S. K. Sinha, and J. D. Axe, "Neutron Diffraction Study of the Pore Surface Layer of Vycor Glass," Phys. Rev. E 55 (1997) 3021-3027

Badyal, Y.S., M.-L. Saboungi, D.L. Price, D.R. Haeffner, and S.D. Shastri, "Atomic and Electronic Structure of Liquid Iron Trichloride," Europhys. Lett. 39 (1997) 19-24

Basdogan, I., T. J. Royston, J. Barraza, D. Shu, and T. M. Kuzay, "A Theoretical and Experimental Study of the Vibratory Behavior of a High-Precision Optical Positioning Table," Proc. of DETC'97, 1997 ASME Design Engineering Technical Conferences, DETC97/VIB-4215 (1997) 1-11

Basdogan, I., T. J. Royston, A. A. Shabana, J. Barraza, D. Shu, and T. M. Kuzay, "Analysis of High-Precision Optical Positioning Systems for Vibration Stability at the Advanced Photon Source," SPIE Vol. 3132 (1997) 47-55

Cai, Z., B. Lai, W. Yun, E. Gluskin, D. Legnini, P. Ilinski, E. Trakhtenberg, S. Xu, W. Rodrigues, H-R. Lee, "Beam Size Measurement of the Stored Electron Beam at the APS Storage Ring Using Zone Plate Optics and Undulator Radiation,” AIP Conf. Proc. 417 (1997) 101

Experimental Facilities Division Progress Report 1996-97, Argonne National Laboratory Report ANL/APS/TB-30 (April 1997)

Feng, Y. P., I. McNulty, Z. Xu, and E. Gluskin, "Signal-to-Noise Ratio of Intensity Interferometry Experiments with Highly Asymmetric X-ray Source," Argonne National Laboratory Light Source Note LS-258 (1997)

Fernandez, P. B., T. Graber, W.-K. Lee, D. M. Mills, C. S. Rogers, and L. A. Assoufid, "Test of a High-Heat-Load Double-Crystal Diamond Monochromator at the Advanced Photon Source," Nucl. Instrum. Meth. Phys. Res. A 400 (1997) 476-483

Fernandez, P. B., T. Graber, S. Krasnicki, W.-K. Lee, D. M. Mills, C. S. Rogers, and L. A. Assoufid, "Test Results of a Diamond Double-Crystal Diamond Monochromator at the Advanced Photon Source," Synchrotron Radiation Instrumention: Tenth U.S. National Conference, ed. E. Fontes (American Institute of Physics, 1997) pp.89-94

Fultz, B., C. C. Ahn, E. E. Alp, W. Sturhahn, and T. S. Toellner, "Phonons in Nanocrystalline ${ }^{57}$ Fe," Phys. Rev. Lett. 79 (1997) 937-940

Fultz, B., T. A. Stephens, W. Sturhahn, T. S. Toellner, and E. E. Alp, "Local Chemical Environments and the Phonon Partial Densities of States of ${ }^{57} \mathrm{Fe}$ in ${ }^{57} \mathrm{Fe}_{3} \mathrm{Al}$," Phys. Rev. Lett. 80 (1998) 3304-3307 
Graber, T., S. Krasnicki, P. B. Fernandez, D. M. Mills, Q.-Y. Tong, and U. M. Gösele, "Progress in Silicon-to-Silicon Direct Bonding and its Application to Synchrotron X-ray Optics,” SPIE Vol. 3151 (1997) 54-64

Hahn, U., H. Schulte-Schrepping, K. Balewski, J. R. Schneider, P. Ilinski, B. Lai, W. Yun, D. Legnini, E. Gluskin, "Measurements of Emittance and Absolute Spectral Flux of the PETRA Undulator at DESY Hamburg," J. Synchrotron Rad. 4 (1997) 1

Hoffberg, M., R. Laird, F. Lenkzsus, C. Liu, B. Rodricks, and A. Gelbart, "The Development of a HIgh-Speed 100 fps CCD Camera," Nucl. Instrum. Meth. Phys. Res. A 392 (1997) 214-219

Ilinski, P.,’Undulator A Diagnostics at the Advanced Photon Source," Argonne National Laboratory Report ANL/APS/TB-33 (1998)

Ilinski, P., C. T. Venkataraman, J. C. Lang, and G. Srajer, "Characterization of the Elliptical Multipole Wiggler at the Advanced Photon Source," Synchrotron Radiation Instrumention: Tenth U.S. National Conference, ed. E. Fontes (American Institute of Physics, 1997) pp.49-54

Ishimatsu, N., C. T. Venkataraman, H. Hashizume, N. Hosoito, K. Namikawa, and T. Iwazumi, "X-ray Reflectivity at the L Edges of Gd," J. Synchrotron Rad. 4 (1997) $175-179$

Kushnir, V. I., P. M. Abbamonte, A. T. Macrander, and M. Schwoerer-Böhning, "Backscattering Channel-Cut High Resolution Monochromator for Inelastic X-ray Scattering," SPIE Vol. 3151 (1997) 324-328

Lal, J., S. K. Sinha, and L. Auvray, "Structure of Polymer Chains Confined in Vycor," J. de Physique II 7 (1997) 1597

Lee, H.-R, "A Novel Iterative Optimizing Quantization Technique for Limited Data Computed Tomography," Optical Engineering Monthly Journal, April 1997

Lee, H.-R., B. Lai, W. Yun, D. Mancini, Z. Cai, "X-Ray Microtomography as a Fast Three-Dimensional Imaging Technology using a CCD Camera Coupled with a $\mathrm{CdWO}_{4}$ Single-Crystal Scintillator," SPIE Vol. 3149 (1997) 257

Lee, H.-R., W. Yun, Z. Cai, W. Rodrigues, D. S. Kupperman, "X-ray Microdiffraction Studies to Measure Strain Fields in a Metal Matrix Composite," AIP Conf. Proc. 417 (1997) 166 
Lee, W.-K., P. B. Fernandez, A. Khounsary, W. Yun, and E. Trakhtenberg, "Advanced Photon Source Undulator Beamline Tests of a Contact-Cooled Silicon U-Shaped Monochromator," SPIE Vol. 3151 (1997) 208-215

Liu, C., D. Shu, T. M. Kuzay, R. Wen, and C. A. Melendres, "Ion Beam Induced Surface Modification of Chemical Vapor Deposition Diamond for X-ray Beam Position Monitor Applications,” J. Vac. Sci. Technol. A 15 (1997) 1200-1205

Lynn, J. W., S. Skanthakumar, Q. Huang, S. K. Sinha, Z.Hossain, L. C. Gupta, R. Nagerajan, and C. Godart, "Magnetic Order and Crystal Structure in the Superconducting $R \mathrm{Ni}_{2} \mathrm{~B}_{2} \mathrm{C}$ Materials," Phys. Rev. B 55 (1997) 6584

Macrander, A. T., M. Schwoerer-Böhning, P. M. Abbamonte, and M. Hu, "High Resolution Monochromator for Inelastic Scattering Studies of High Energy Phonons Using Undulator Radiation at the Advanced Photon Source," SPIE Vol. 3151 (1997) 271-281

Makarov, O. A., P. Den Hartog, E. R. Moog, and M. L. Smith, "Control System for Insertion Devices at the Advanced Photon Source," Synchrotron Radiation Instrumention: Tenth U.S. National Conference, ed. E. Fontes (American Institute of Physics, 1997) pp.43-47

Mills, D. M., "X-ray Optics Developments at the APS for the Third Generation of HighEnergy Synchrotron Radiation Sources," J. Synchrotron Rad. 4 (1997) 117-124

Mochrie, S. G. J., A. M. Mayes, A. R. Sandy, M. Sutton, S. Brauer, G. B. Stephenson, D. L. Abernathy, and G. Grubel, "Dynamics of Block Copolymer Micelles Revealed by X-ray Intensity Fluctuation Spectroscopy,” Phys. Rev. Lett. 78 (1997) 12751278

Moog, E. R., P. K. Den Hartog, E. J. Semones, and P. K. Job, "Radiation Doses to Insertion Devices at the Advanced Photon Source," Synchrotron Radiation Instrumention: Tenth U.S. National Conference, ed. E. Fontes (American Institute of Physics, 1997) pp. 219-223

Mui, P. H., G. Srajer, and D. M. Mills, "In-situ Surface Monitoring System for Synchrotron Mirrors under High Heat Load," Applied Optics 36 (1997) 5546-5551

Ocko, B. M., X. Z. Wu, E. B. Sirota, S. K. Sinha, O. Gang, and M. Deutsch, "Surface Freezing in Chain Molecules: I. Normal Alkanes," Phys. Rev. E 55 (1997) 3164

Pisharody, M., P. K. Job, S. Magill, J. Proudfoot, and R. Stanek, "Measurement of Gas Bremsstrahlung from Electron Storage Rings," Nucl. Instrum. Meth. Phys. Res. A 401 (1997) 442-462 
Pisharody, M., P. K. Job, S. Magill, J. Proudfoot, and R. Stanek, "Measurement of Gas Bremsstrahlung from the Insertion Device Beamlines of the Advanced Photon Source," Argonne National Laboratory Light Source Note LS-260 (1997)

Pisharody, M., E. Semones, and P. K. Job, "Dose Measurements of BremsstrahlungProduced Neutrons at the Advanced Photon Source," Argonne National Laboratory Light Source Note LS-269 (1998)

Pisharody, M., P. K. Job, S. Magill, J. Proudfoot, and R. Stanek, "Measurement of Gas Bremsstrahlung from the Insertion Device Beam Lines of the Advanced Photon Source," Proceedings of the Third Specialists Meeting on Shielding Aspects of Accelerators, Targets and Irradiation Facilities (Nuclear Energy Agency, OECD Publications, Paris, France, 1998) pp. 33-40

Randall, K. J., Z. Xu, J. F. Moore, and E. Gluskin, "Soft X-ray Spectroscopy Undulator Beamline at the Advanced Photon Source," SPIE Vol. 3150 (1997) 189-194

Rodrigues, W., Z. Cai, W. Yun, H-R. Lee, P. Ilinski, E. Isaacs, J. Grenko, "X-ray Microdiffraction Studies of an Integrated Laser-Modulator System," AIP Conf. Proc. 417 (1997) 161

Röhlsberger, R., E. Gerdau, R. Rüffer, W. Sturhahn, T. S. Toellner, A. I. Chumakov, and E. E. Alp, "X-ray Optics for $\mu \mathrm{eV}$-Resolved Spectroscopy," Nucl. Instrum. Meth. Phys. Res. A 394 (1997) 251-255

Rosenkranz, S., R. Osborn, J. F. Mitchell, L. Vasiliu-Doloc, J.W. Lynn, S. K. Sinha, and D. N. Argyriou, "Magnetic Correlations in the Bilayer Manganite $\mathrm{La}_{1,2} \mathrm{Sr}_{1.8} \mathrm{Mn}_{2} \mathrm{O}_{7}, "$ J. Appl. Phys. 83 (1998) 7348

Sanchez del Rio, M., and R. J. Dejus, "XOP: A Multiplatform Graphical User Interface for Synchrotron Radiation Spectral and Optics Calculations," SPIE Vol. 3152 (1997) 148-157

Schwoerer-Böhning, M., P. M. Abbamonte, A. T. Macrander, and V. I. Kushnir, "A Spherical Bent Focusing Analyzer for High Resolution Inelastic X-ray Scattering," SPIE Vol. 3151 (1997) 282-286

Schwoerer-Böhning, M., A. T. Macrander, and D. A. Arms, "Phonon Dispersion of Diamond Measured by Inelastic X-ray Scattering," Phys. Rev. Lett. 80 (1998) $5572-5575$

Schwoerer-Böhning, M., A. T. Macrander, P. M. Abbamonte, , and D. A. Arms, "High Resolution Inelastic X-ray Scattering Spectrometer at the Advanced Photon Source,” Rev. Sci. Instrum. 69 (1998) 3109-3112 
Shastri, S.D, R. J. Dejus, and D. R. Haeffner, "Experimental Characterization of APS Undulator A at High Photon Energies (50-200 keV)," J. Synchrotron Rad. 5 (1998) $67-71$

Shu, D., C. Benson, J. Chang, J. Barraza, T. M. Kuzay, E. E. Alp, W. Sturhahn, B. Lai, I. McNulty, K. Randall, G. Srajer, Z. Xu, and W. Yun, "Mirror Mounts Designed for the Advanced Photon Source SRI-CAT," Synchrotron Radiation Instrumention: Tenth U.S. National Conference, ed. E. Fontes (American Institute of Physics, 1997) pp.179-185

Sinha, S. K., and R. Pynn, "Diffuse X-ray and Neutron Reflection from Surfaces and Interfaces," in Local Structure from Diffraction, eds. S. J. L. Billinge and M. F. Thorpe (Plenum Press, New York, 1998)

Sinha, S. K., M. Tolan, and A. Gibaud, "Effects of Partial Coherence on the Scattering of X-rays by Matter,” Phys. Rev. B 57 (1998) 2740-2758

Smither, R. K., A. M. Khounsary, and S. Xu, "Potential of a Beryllium X-ray Lens," SPIE Vol. 3151 (1997) 150-163

Sutter, J., E. Alp, J. Barraza, and D. Shu, "Vibrational Measurements in 3-ID-B," Argonne National Laboratory Light Source Note LS-265 (1998)

Toellner, T. S., M. Y. Hu, W. Sturhahn, K. Quast, and E. E. Alp, "Inelastic Nuclear Resonant Scattering with Sub-meV Energy Resolution," Applied Physics Letters 71 (1997) 2112-2114

Tolan, M., and S. K. Sinha, "X-ray Scattering with Partially Coherent Radiation: The Exact Relationship between Resolution and Coherence," Physica B 248 (1998) 399

Tolan, M., O. H. Seeck, J.-P Schlomka, W. Press, J. Wang, S. K. Sinha, Z. Li, M. Rafailovich, and J. Sokolov, "Evidence for Capillary Waves on Dewetted Polymer Film Surfaces: A Combined X-ray and Atomic Force Microscopy Study," Phys. Rev. Lett. 81 (1998) 2731

Ulmer, M., R. Altkorn, A. Krieger, D. Parsignault, Y. Chung, M. Wong, B. Lai, D. Mancini, P. Takacs, E. Church, "Super Mirror Fabrication via Electroforming," SPIE Vol. 3153 (1997) 240

Vasserman, I., "Test of Horizontal Field Measurements Using Two-Axis Hall Probes at the APS Magnetic Measurement Facility," Argonne National Laboratory Report ANL/APS/TB-32 (1998)

Venkataraman, C. T., J. C. Lang, C. S. Nelson, G. Srajer, D. R. Haeffner, and S. D. Shastri, "A High Energy Phase Retarder for the Simultaneous Production of Right- 
and Left-Handed Circularly Polarized X-rays,” Rev. Sci. Instrum. 69 (1998) 19701973

Vignaud, G., A. Gibaud, J. Wang, S. K. Sinha, J. Daillant, G. Grubel, and Y. Gallot, “An X-ray Scattering Study of Laterally Modulated Structures: The Example of Diblock Copolymers," J. Phys.: Condens. Matter 9 (1997) L125

Vinokurov, N. A., R. J. Dejus, H. Friedsam, E. S. Gluskin, J. Maines, S. V. Milton, E. R. Moog, E. M. Trakhtenberg, and I. B. Vasserman, "Design Considerations for the Magnetic System of a Prototype X-ray Free Electron Laser,” SPIE Vol. 2988 (1997) 64-68

Wang, J., A. K. Sood, P. V. Satyam, Y. Feng, X.-Z. Wu, Z. Cai, W. Yun, and S. K. Sinha, "X-ray Fluorescence Correlation Spectroscopy: A Method for Studying Particle Dynamics in Condensed Matter," Phys. Rev. Lett. 80 (1998) 1110-1113

Wang, J., "Characterizing Surfaces and Interfaces Using X-ray Standing Waves," Invited paper to be published in Current Opinion in Colloid and Interface Science, Vol. $3 / 3,1998$

Xu, Z. and K. J. Randall, "Virtual Sine Arm Kinematic Mount System,” SPIE Vol.3150 (1997) 183-188

Zhao, M., D. S. Dmitriy, Z. Cai, and S. A. Rice, "Structure of Liquid Ga and the LiquidVapor Interface of Ga, Phys. Rev. E 56 (1997) 7033-7042

\section{Patent Application}

Bajiakar, S. S., Francesco De Carlo, Joshua J. Song, "Enhanced adhesion for LIGA microfabrication by using a buffer layer," Department of Energy Patent Application Number S-89, 870, May 1998 


\section{7-1998 Invited Presentations by XFD Staff}

Alp, E. E., American Physical Society, March Meeting, March 17-21,1997, Kansas City, MO

Alp, E. E., Eighth Users Meeting for the Advanced Photon Source, April 15-17,1997, Argonne National Laboratory, IL

Alp, E. E., Crystallographic Applications of Synchrotron Radiation, July 31-August 1,1997, KEK-Tsukuba, Japan

Alp, E. E., Inelastic Scattering Probes of Condensed Matter, University of Chicago, May 13-15, 1998, Chicago, IL

Alp, E. E., Free Electron Laser Workshop, Argonne National Laboratory, IL

Alp, E. E., SPIE, Time Resolved Studies Symposium July 20-24, 1998, San Diego, CA

Alp, E. E., HFI-11, International Conference on Hyperfine Interactions, Aug 24-28,1998, Durban, South Africa

Alp, E. E., International Workshop on Inelastic X-ray Scattering, October 19-21, 1998, Montauk, NY

Alp, E. E., 2nd International Conference on Synchrotron Radiation in Material Science, October 31-November 3,1998, Kobe, Japan

Cai, Z., Studies of Strain Field in EML Devices and Electromigration in Thin Al/Cu Conduct Wires Using an X-ray Microscope, Scanning 98 (Tenth Annual International Meeting on Scanning Microscopies), May 11, 1998, Baltimore, MD

Fernandez, P.B., Test Results of a Diamond Double-Crystal Monochromator at the Advanced Photon Source, Synchrotron Radiation Instrumentation: Tenth US National Conference, Cornell University, Ithaca, NY (1997)

Lai, B., Capabilities and Applications of an Advanced Scanning X-ray Microprobe, Scanning 98 (Tenth Annual International Meeting on Scanning Microscopies), May 11, 1998, Baltimore, MD

Lai, B., Capabilities of X-ray Microprobe at the Advanced Photon Source, Principal Residual Stress Workshop, July 28, 1997, Los Alamos National Laboratory, NM

Moog, L., Magnetic Performance of Insertion Devices at the Advanced Photon Source, Particle Accelerator Conference (PAC 97), May 12-165, 1997, Vancouver, Canada 
Moog, L., Insertion Devices at the Advanced Photon Source, November 1997 monthly meeting of the Joint Chicago Chapter of the IEEE Nuclear and Plasma Sciences Society and the IEEE Magnetics Society, Nov. 1997, Chicago, IL

Mooney, T., Overview of EPICS-based Beamline Software, New Opportunities forBetter User Group Software (NOBUGS '97), Dec. 10-12, 1997, Argonne National Laboratory

Shenoy, G. K., 7-GeV Advanced Photon Source, Fermi National Accelerator Laboratory, Sept 24, 1997, Batavia, IL

Shenoy, G. K., 7-GeV Advanced Photon Source, Ceramic Society, June 25, 1998, Argonne National Laboratory

Shenoy, G. K., Possible Application of the APS to the Rotating Equipment Industry, Rotating Equipment Consortium, Sept. 30, 1998, Argonne National Laboratory

Shu, D., a series of 20 lectures on synchrotron radiation beamline design topics at the Beijing Synchrotron Radiation Facility (BSRF), Institute of High Energy Physics (IHEP), Chinese Academy of Sciences, May 8-12, 1998, Beijing, China

Shu, D., APS Synchrotron Radiation Beamline Front-End Design, Shanghai Institute for Nuclear Research, Chinese Academy of Sciences, May 13, 1998, Shanghai, China

Sinha, S., X-Ray Studies of Surface Phase Transitions, Penn State U. Physics Colloquium, May 7, 1997,

Sinha, S., Review of Condensed Matter Studies Using Synchrotron Radiation, (Birgeneau Panel) BESAC Sub-Committee on X-Ray Sources, May 10, 1997

Sinha, S., X-Ray Scattering from Condensed Matter Using Coherent Beams, 5th International Conference onSurface X-Ray and Neutron Scattering, July 18, 1997, Daresbury Laboratory, U.K.

Sinha, S., Synchrotron Radiation Studies of the Structure and Dynamics of Complex Fluid Films, European Physical Society Meeting, August 26, 1997, Leuwen, Belgium

Sinha, S., X-Ray Fluorescent Correlation Spectroscopy - a New Method of Studying Slow Dynamics, XFEL Workshop, October 28, 1997, APS, Argonne National Laboratory

Sinha, S., The Use of Coherent, Partially Coherent and Incoherent Radiation to Study Fluctuations In Condensed Matter, SR-50 Meeting (Highlights of Synchrotron Radiation Research), November 18, 1997

Sinha, S., The Use of Partially Coherent Radiation to Study Condensed Matter, Purdue University, Physics Colloquium, Feb. 12, 1998, Lafayette, IN 
Sinha, S., Frontiers of Materials Science using Synchrotron Radiation, Johns Hopkins University Physics Colloquium, Mar. 26, 1998, Baltimore, MD

Sinha, S., Condensed Matter Studies using Coherent and Microfocused X-ray Beams, University of Wisconsin Physics Colloquium, April 3, 1998, Milwaukee, WI

Sinha, S., X-ray Diffuse Scattering as a Probe for the Study of Structural and Magnetic Roughness in Metallic Multilayers, 3rd International Symposium on Metallic Multilayers, June 14-19, 1998, Vancouver, BC

Sinha, S., Synchrotron Radiation Studies of Liquid Polymer Films, American Crystallographic Association, July 18-23, 1998, Arlington, VA

Sinha, S., Neutron X-ray Scattering from Porous Media Fractals and Rough Surfaces, $6^{\text {th }}$ International Summer School on Neutron Scattering, Aug. 8-14, 1998, Zuoz, Switzerland

Sinha, S., New Developments in the Application of Synchrotron Radiation to Materials Science, Plenary Lecture SRMS-2 Conference, Oct. 31-Nov. 3, 1998, Kobe, Japan

Srajer, G., Magnetic Reflectivity Measurements in Fe/Gd Multilayers, Conference on "X-rays in Magnetism," July 1998, Daresbury Laboratory, U.K.

Sturhahn, W., Inelastic Nuclear Resonant Scattering: Technique and Data Evaluation, Workshop on Inelastic Nuclear Resonant Scattering, April 21 - 22, 1997, Argonne National Laboratory

Sturhahn, W., Inelastic Nuclear Resonant Scattering, Gordon Conference on X-ray Physics, August 3-8, 1997, Plymouth, NH

Sturhahn, W., Introduction to Nuclear Resonant Scattering with Synchrotron Radiation, International Conference on the Applications of the Mossbauer Effect, Sept. 14 - 20, 1997, Rio de Janeiro, Brazil,

Sturhahn, W., Vibrational Dynamics of Thin Films by Inelastic Nuclear Resonant Scattering of Synchrotron Radiation, International Conference on the Physics of X-ray Multilayer Structures, Mar. 1 - 5, 1998, Breckenridge, CO

Sturhahn, W., Vibrational Density of States Obtained from Inelastic Nuclear Resonant Absorption of Synchrotron Radiation, Ninth International Conference on Phonon Scattering in Condensed Matter, July 26-31, 1998, Lancaster, U.K. 


\section{SRI-CAT Staff and Members}

\section{APS-Experimental Facilities Division (XFD) Developers}

\begin{tabular}{|l|l|l|l|}
\hline E. Alp & Z. Cai & P. Den Hartog & M. Erdmann \\
\hline P. Fernandez & E. Gluskin & D. Haeffner & P. Ilinski \\
\hline T. Kuzay & B. Lai & J. Lang & P. Lee \\
\hline W. K. Lee & D. Legnini & D. C. Mancini & J. Maser \\
\hline W. McHargue & I. McNulty & A. McPherson & D. M. Mills \\
\hline T. Mooney & K. Randall & S. Shastri & D. Shu \\
\hline S. Sinha & R. Smither & G. Srajer & W. Sturhahn \\
\hline B. Tieman & T. Toellner & C. Venkataraman & J. Wang \\
\hline G. Wiemerslage & W. Yun & & \\
\hline
\end{tabular}

\section{APS External Developers}

\begin{tabular}{|l|l|l|l|}
\hline J. Arthur & SSRL & D. Bilderback & CHESS \\
\hline R. Colella & Purdue & S. Durbin & Purdue \\
\hline T. Jach & NIST & S. Moss & U-Houston \\
\hline A. Thompson & LBNL & Q. Shen & CHESS \\
\hline A. Stampfl & Australia/ASRP & & \\
\hline
\end{tabular}

\section{Scientific Members}

\begin{tabular}{|l|l|l|l|}
\hline T. W. Barbee & LLNL & F. Cerrina & Univ. of Wisc./Madison \\
\hline C. T. Chen & SRRC, Taiwan & T. C. Chiang & Univ. of Illinois \\
\hline R. Deslattes & NIST & D. Ederer & Tulane Univ. \\
\hline B. Fultz & Cal. Inst. of Tech. & M. Howells & ALS/LBNL \\
\hline G. Ice & ORNL & E. Isaacs & Lucent Technologies \\
\hline N. Ishimatsu & Tokyo Inst. of Tech. & C. Jacobson & SUNY/Stony Brook \\
\hline J. Kirz & SUNY/Stony Brook & O. Leupold & Univ. of Hamburg \\
\hline P. Montano & ANL/MSD & J. Mullen & Purdue University \\
\hline G. Neyes & Katholieke Univ. Leuven & P. Platzman & AT\&T Bell Labs \\
\hline R. Simmons & Univ. of Illinois & J. Stohr & IBM/Almaden \\
\hline J. Tischler & ORNL & J. Tobin & LLNL \\
\hline J. Trebes & LLNL & & \\
\hline
\end{tabular}


Review Status of APS Collaborative Access Teams*

\begin{tabular}{|c|c|c|c|c|c|c|c|c|c|}
\hline \multirow{2}{*}{$\begin{array}{c}\text { CAT } \\
\text { Acronym }\end{array}$} & \multirow{2}{*}{$\begin{array}{c}\text { CAT } \\
\text { Director }\end{array}$} & \multirow{2}{*}{$\begin{array}{c}\text { Sector } \\
\text { Assignment }\end{array}$} & \multicolumn{2}{|c|}{ Letter of Intent } & \multicolumn{2}{|c|}{ Proposal } & \multicolumn{2}{|c|}{ Management Plan } & \multirow{2}{*}{$\begin{array}{c}\text { Funding } \\
\text { Letters } \\
\text { Received } \\
\end{array}$} \\
\hline & & & Submitted & Accepted & Submitted & Accepted & Submitted & Accepted & \\
\hline BESSRC & Pedro Montano & 11,12 & $5 / 1 / 90$ & $6 / 12 / 90$ & $\begin{array}{l}3 / 15 / 91 \\
3 / 31 / 93\end{array}$ & $\begin{array}{l}11 / 1 / 91 \\
5 / 14 / 93\end{array}$ & $3 / 21 / 94$ & $7 / 5 / 94$ & Received \\
\hline $\mathrm{BIO}$ & Grant Bunker & 18 & $5 / 1 / 90$ & $6 / 12 / 90$ & $3 / 15 / 91$ & $11 / 1 / 91$ & $11 / 14 / 95$ & $1 / 3 / 96$ & $12 / 1 / 95$ \\
\hline CARS & J. Keith Moffat & $13,14,15$ & $\begin{array}{c}5 / 1 / 90 \\
1 / 15 / 92\end{array}$ & $\begin{array}{l}6 / 12 / 90 \\
3 / 31 / 92\end{array}$ & $\begin{array}{l}3 / 15 / 91 \\
3 / 31 / 93 \\
8 / 13 / 93\end{array}$ & $\begin{array}{l}11 / 1 / 91 \\
5 / 14 / 93 \\
9 / 30 / 93\end{array}$ & $11 / 22 / 93$ & $3 / 14 / 94$ & $\begin{array}{c}4 / 5 / 94 \\
9 / 19 / 96\end{array}$ \\
\hline $\mathrm{CMC}$ & L. Doon Gibbs & 9 & $5 / 1 / 90$ & $6 / 12 / 90$ & $3 / 15 / 91$ & $11 / 1 / 91$ & $5 / 26 / 94$ & $10 / 28 / 94$ & $3 / 9 / 95$ \\
\hline COM & K. L. D'Amico & 32 & $1 / 14 / 97$ & $4 / 29 / 97$ & $5 / 28 / 97$ & $7 / 7 / 97$ & $6 / 11 / 98$ & & $8 / 98$ \\
\hline DND & John Quintana & 5 & $\begin{array}{c}5 / 1 / 90 \\
12 / 15 / 91\end{array}$ & $\begin{array}{l}6 / 12 / 90 \\
1 / 30 / 92\end{array}$ & $3 / 15 / 91$ & $4 / 1 / 92$ & $1 / 6 / 93$ & $6 / 16 / 93$ & $8 / 3 / 93$ \\
\hline IMCA & Andrew Howard & 17 & $5 / 1 / 90$ & $6 / 12 / 90$ & $3 / 15 / 91$ & $11 / 1 / 91$ & $2 / 20 / 94$ & $5 / 24 / 94$ & $12 / 15 / 93$ \\
\hline IMM & Simon Mochrie & 8 & $5 / 1 / 90$ & $6 / 12 / 90$ & $3 / 15 / 91$ & $11 / 1 / 91$ & $2 / 22 / 94$ & $4 / 8 / 94$ & $\begin{array}{l}2 / 22 / 94 \\
4 / 18 / 94\end{array}$ \\
\hline MHATT & Roy Clarke & 7 & $5 / 1 / 90$ & $6 / 12 / 90$ & $3 / 15 / 91$ & $11 / 1 / 91$ & $11 / 29 / 93$ & $5 / 25 / 94$ & $6 / 1 / 94$ \\
\hline MR & Bruce Bunker & 10 & $5 / 1 / 90$ & $6 / 12 / 90$ & $3 / 15 / 91$ & 4/ 1/ 92 & $7 / 19 / 94$ & $11 / 21 / 94$ & $11 / 28 / 94$ \\
\hline$\mu$ & Alan Goldman & 6 & $5 / 1 / 90$ & $6 / 12 / 90$ & $3 / 15 / 91$ & 4/1/92 & $6 / 6 / 95$ & $6 / 20 / 96$ & $\begin{array}{c}6 / 5 / 96 \\
6 / 26 / 96\end{array}$ \\
\hline PNC & Edward Stern & 20 & $5 / 1 / 90$ & $6 / 12 / 90$ & $3 / 15 / 91$ & $4 / 1 / 92$ & $6 / 6 / 94$ & $6 / 28 / 94$ & $12 / 16 / 94$ \\
\hline SBC & $\begin{array}{l}\text { Andrzej } \\
\text { J oachimiak }\end{array}$ & 19 & $5 / 1 / 90$ & $6 / 12 / 90$ & $3 / 15 / 91$ & $4 / 1 / 92$ & $12 / 22 / 93$ & $3 / 14 / 94$ & $3 / 94$ \\
\hline SG & K. L. D'Amico & & $5 / 14 / 97$ & $5 / 30 / 97$ & $5 / 30 / 97$ & $7 / 7 / 97$ & & & \\
\hline SRI & Dennis Mills & $1,2,3,4$ & $5 / 1 / 90$ & $6 / 12 / 90$ & $3 / 15 / 91$ & $3 / 31 / 92$ & $12 / 22 / 93$ & $2 / 16 / 94$ & Received \\
\hline UNI & Haydn Chen & 33,34 & $5 / 1 / 90$ & $6 / 12 / 90$ & $\begin{array}{c}3 / 15 / 91 \\
(\mathrm{sec} .33) \\
4 / 4 / 96 \\
(\mathrm{sec} .34)\end{array}$ & $\begin{array}{c}11 / 1 / 91 \\
3 / 97\end{array}$ & $7 / 21 / 93$ & $8 / 23 / 93$ & $4 / 21 / 94$ \\
\hline
\end{tabular}

* Note that Letters of Intent have been received and accepted from four additonal CATs, and Proposals have been received from three of these. 


\begin{tabular}{|c|c|c|c|c|c|c|c|c|c|}
\hline \multirow{2}{*}{$\begin{array}{c}\text { CAT } \\
\text { Acronym }\end{array}$} & \multicolumn{2}{|c|}{$\begin{array}{c}\text { Conceptual Design } \\
\text { Report }\end{array}$} & \multirow{2}{*}{$\begin{array}{c}\text { Memorandum of } \\
\text { Underst a nd in } \\
\text { Signed }\end{array}$} & \multicolumn{2}{|c|}{$\begin{array}{c}\text { Preliminary } \\
\text { Design Report }\end{array}$} & \multicolumn{2}{|c|}{ Final Design Report } & \multicolumn{2}{|c|}{ Safety Plan } \\
\hline & Submitted & Approved & & Submitted & Approved & Submitted & Approved & Submitted & Approved \\
\hline BESSRC & $11 / 1 / 92$ & $7 / 28 / 93$ & $7 / 6 / 94$ & $2 / 17 / 95$ & $5 / 22 / 95$ & $\begin{array}{c}10 / 31 / 95 \\
(12 \mathrm{BM}) \\
4 / 18 / 96 \\
(12 \text { ID })\end{array}$ & $\begin{array}{c}7 / 23 / 96 \\
(12 \mathrm{BM} \\
\text { and } \\
12 \text { ID) }\end{array}$ & $3 / 15 / 96$ & $6 / 6 / 96$ \\
\hline $\mathrm{BIO}$ & $1 / 94$ & $5 / 10 / 94$ & $12 / 4 / 95$ & $7 / 23 / 96$ & $12 / 20 / 96$ & $5 / 20 / 97$ & $1 / 9 / 98$ & $6 / 19 / 96$ & $8 / 29 / 96$ \\
\hline CARS & $\begin{array}{c}11 / 1 / 92 \\
\text { (2 sectors) } \\
10 / 1 / 93 \\
\text { (3rd sec.) }\end{array}$ & $\begin{array}{l}1 / 14 / 93 \\
1 / 13 / 94\end{array}$ & $\begin{array}{c}4 / 27 / 94 \\
(\text { sec. } 13,14) \\
10 / 21 / 96 \\
\text { (sec. 15) }\end{array}$ & $\begin{array}{c}11 / 4 / 94 \\
(\mathrm{sec} .14) \\
12 / 12 / 95 \\
\text { (sec. 13) }\end{array}$ & $\begin{array}{l}12 / 15 / 94 \\
5 / 21 / 96\end{array}$ & $\begin{array}{c}7 / 17 / 95 \\
(\text { sec. } 14) \\
10 / 21 / 96 \\
\text { (sec. 13) }\end{array}$ & $\begin{array}{c}12 / 12 / 95 \\
(\mathrm{sec} .14) \\
10 / 96 \\
(\mathrm{sec} .13)\end{array}$ & $5 / 1 / 96$ & $6 / 12 / 96$ \\
\hline CMC & $8 / 14 / 92$ & $1 / 7 / 93$ & $10 / 31 / 94$ & $9 / 20 / 95$ & $2 / 7 / 96$ & & & $5 / 26 / 94$ & $10 / 28 / 94$ \\
\hline COM & $6 / 18 / 98$ & & & & & & & & \\
\hline DND & $11 / 1 / 92$ & $7 / 28 / 93$ & $12 / 15 / 93$ & $12 / 5 / 94$ & $4 / 19 / 94$ & $7 / 19 / 95$ & $11 / 3 / 95$ & $11 / 11 / 95$ & $5 / 28 / 96$ \\
\hline IMCA & $11 / 1 / 92$ & $7 / 28 / 93$ & $9 / 16 / 94$ & $10 / 20 / 95$ & $1 / 30 / 96$ & $4 / 2 / 97$ & $10 / 30 / 97$ & $5 / 8 / 96$ & $7 / 25 / 96$ \\
\hline IMM & $10 / 2 / 93$ & $1 / 13 / 94$ & $5 / 19 / 94$ & $8 / 2 / 95$ & $1 / 10 / 96$ & $7 / 15 / 96$ & $10 / 96$ & $5 / 30 / 96$ & $8 / 15 / 96$ \\
\hline MHATT & $11 / 1 / 92$ & $1 / 7 / 93$ & $6 / 2 / 94$ & $2 / 6 / 96$ & $7 / 23 / 96$ & $11 / 97$ & $3 / 98$ & $11 / 29 / 93$ & $5 / 25 / 94$ \\
\hline MR & $11 / 92$ & $7 / 28 / 93$ & $5 / 24 / 95$ & $2 / 8 / 96$ & $7 / 23 / 96$ & $11 / 97$ & $1 / 98$ & $5 / 23 / 96$ & $7 / 19 / 96$ \\
\hline$\mu$ & $11 / 92$ & $1 / 14 / 93$ & $8 / 27 / 96$ & $5 / 1 / 96$ & $1 / 98$ & & & $6 / 9 / 95$ & $6 / 20 / 96$ \\
\hline PNC & $8 / 7 / 92$ & $1 / 14 / 93$ & $5 / 11 / 95$ & $\begin{array}{l}6 / 14 / 95 \\
\quad(\text { ID) }\end{array}$ & $\begin{array}{l}9 / 19 / 95 \\
\text { (ID) }\end{array}$ & $\begin{array}{c}12 / 19 / 96 \\
\text { (ID) }\end{array}$ & $\begin{array}{c}11 / 28 / 97 \\
\text { (ID) }\end{array}$ & $8 / 14 / 96$ & $10 / 7 / 96$ \\
\hline SBC & $11 / 1 / 92$ & $7 / 28 / 93$ & $3 / 17 / 94$ & $6 / 22 / 95$ & $9 / 22 / 95$ & $\begin{array}{l}2 / 9 / 96 \\
(\text { ID) } \\
4 / 24 / 97 \\
\text { (BM) }\end{array}$ & $\begin{array}{l}7 / 8 / 96 \\
\text { (ID) } \\
1 / 98 \\
\text { (BM) }\end{array}$ & $1 / 23 / 96$ & $3 / 12 / 96$ \\
\hline \multicolumn{10}{|l|}{ SG } \\
\hline SRI & $11 / 1 / 92$ & $1 / 14 / 93$ & $4 / 29 / 94$ & $7 / 25 / 94$ & $10 / 15 / 94$ & $\begin{array}{l}2 / 2 / 95 \\
(\mathrm{sec} .1) \\
3 / 8 / 95 \\
(\mathrm{sec} .3) \\
9 / 20 / 95 \\
\text { (sec. } 2)\end{array}$ & $\begin{array}{c}4 / 24 / 95 \\
(\mathrm{sec} .1) \\
3 / 27 / 95 \\
(\mathrm{sec} .3) \\
10 / 95 \\
(\mathrm{sec} .2)\end{array}$ & $2 / 2 / 95$ & $5 / 17 / 95$ \\
\hline UNI & $8 / 92$ & $1 / 14 / 93$ & $\begin{array}{l}4 / 27 / 94 \\
(\mathrm{sec} .33) \\
8 / 5 / 98 \\
(\mathrm{sec} .34)\end{array}$ & $\begin{array}{c}9 / 6 / 94 \\
(33 \mathrm{ID}) \\
1 / 20 / 97 \\
(33 \mathrm{BM})\end{array}$ & $10 / 21 / 94$ & $\begin{array}{c}4 / 20 / 95 \\
(33 \text { ID) } \\
11 / 19 / 97 \\
\text { (33BM) }\end{array}$ & $\begin{array}{l}8 / 7 / 95 \\
(33 \mathrm{ID0} \\
3 / 98 \\
(33 \mathrm{BM})\end{array}$ & $2 / 12 / 96$ & $8 / 30 / 96$ \\
\hline
\end{tabular}




\section{User Agreement and Proprietary Account Status (8/25/98)}

Listed below are the 143 institutions that have signed APS User Agreements as financially contributing CAT members (indicated by CAT acronyms) and/or as non-members (indicated by "II") as of August 25, 1998. A CAT Agreement covers users' activities at that CAT's beamlines; an II Agreement covers users' activities at any APS beamline. All listed Agreements are nonproprietary unless otherwise noted. A superscript $\mathrm{p}$ indicates that a proprietary User Account has also been established.

Abbott Laboratories (IMCA*,p, II)

Adler Planetarium (II)

Ames Lab $(\mu$, II)

$\operatorname{Amoco}(\mathrm{MR} *, \mathrm{p})$

ANSTO (CARS, SRI)

Bar-Ilan U. (II)

Bayer Corp. (IMCA*)

Bayerisches Geoinstitut (II)

Bede Scientific (II)

Bell Labs/div. of Lucent (MHATT, II)

Biotechnology Research Inst. (II)

Brandeis U. (II)

Bristol-Myers Squibb (IMCA*,p)

Brookhaven National Lab (CMC, II)

California Inst. of Technol. (II)

Carnegie Inst. of Washington/Geophys. Lab (II)

Case Western Reserve U. (II)

CINVESTAV-Merida (II)

Columbia U. (II)

Cornell U. (II)

Daresbury Laboratory (II)

DESY (II) (this Agreement covers HASYLAB)

Digital Equipment Corp. (II)

Dow (DND*,p)

Duke University (II)

DuPont (DND*,p)

DuPont Merck (II*,p)

Eli Lilly (IMCA*,p)

Engineering \& Mgmt. Specialists, Inc. (II*)

ESRF (II)

Exxon (CMC*)

Field Museum (II)

Florida State U. (II)

Forschungszentrum Jülich $(\mu)$

Genentech, Inc. (II*)

Georgia Inst. of Technol. $(\mu)$

Gerhard-Mercator-U. Duisburg/Laboratory of

Applied Physics (II)

Glaxo (IMCA*,p)

Goshen College (II)

Harvard U. (II)

Hebrew U. of Jerusalem (II)
Howard U. (MHATT)

IBM (IMM, II)

Illinois Inst. of Technol. (Bio, IMCA, MR, II)

Indiana U. (II)

Kent State U. $(\mu)$

Korea Electronics Technology Inst. (II)

Kraft Foods Technol. Center (Bio*,p)

Kyoto U. (II)

Laboratoire de Minéralogie-Cristallographie

Paris (II)

Laboratoire Sciences de la Terre ENS Lyon (II)

Lawrence Berkeley National Lab (II)

Lawrence Livermore National Lab (II)

Los Alamos National Lab (CMC, II)

Louisiana State U. (II)

Louisiana Tech. U./Inst. for Micromanufactg. (II)

Massachusetts Inst. of Technol. (IMM)

Max Planck Soc./Res. Unit for StructMolBiol. (II)

Mayo Foundation (II)

McGill U. (IMM)

Merck \& Co., Inc. (IMCA*,p)

Monsanto/Searle (IMCA*)

Naval Research Lab (II)

NCI-Frederick Cancer R\&D Center (II)

New Jersey Institute of Technology (II)

NIST (SRI, UNI, II)

North Carolina State U. (II)

Northeastern U. (II)

Northern Illinois U. (BESSRC, CARS)

Northwestern U. (DND, MR, II)

Oak Ridge Associated Universities (UNI)

Oak Ridge National Lab (CMC, UNI, II)

Ohio State U. (II)

Pacific Northwest National Lab (PNC, II)

Parke-Davis (IMCA*)

Paul Scherrer Inst. (II)

Pennsylvania State U. (II)

Pharmacia \& Upjohn (IMCA*,p)

Photon Factory/KEK (II)

Princeton U. (CMC, II)

Procter \& Gamble (IMCA*,p)

Purdue Research Foundation (SRI) 
Purdue U. (II)

Queens U. (IMM)

Radiation Science, Inc. (II)

Rensselaer Polytechnic Inst. (II)

Rockefeller U. (II)

Rutgers U. (II)

St. Jude Children's Research Hospital (II*)

Schering-Plough Research Inst. (IMCA*,p)

Scripps Research Inst. (II)

Simon Fraser U. (PNC)

SmithKline Beecham (IMCA*,p)

Southern Illinois U. at Carbondale (CARS*, II*)

Stanford U./SSRL (II)

State U. of New York at Stony Brook ( $\mu$, II)

Synchrotron Radiation Research Center (II)

Technische Universität München (II)

Tokyo Inst. of Technol. (II)

Tokyo U. of Science (II)

Tulane U. (II)

U. de Marne la Vallée (II)

U. College London Geological Sciences (II)

U. of Alabama-Birmingham (II*)

U. of California-Berkeley, Dept. of Geology and Geophysics (II)

U. of California-Irvine (II)

U. of California-Santa Barbara (CMC, II)

U. of Chicago (CARS, II)

U. of Florida (MR, II)

U. of Frankfurt (II)

U. of Georgia (II)

U. of Hamburg/Inst. für Experimentalphysik (II)
U. of Houston (II)

U. of Illinois (UNI, II)

U. of Kentucky (II)

U. of Leuven (II)

U. of Manchester (II)

U. of Maryland (II)

U. of Maryland Biotech Inst. (II)

U. of Michigan (MHATT, II)

U. of Minnesota (II)

U. of Missouri-Columbia ( $\mu$, II)

U. of Missouri-Kansas City (II)

U. of New South Wales (II)

U. of North Carolina (II)

U. of Notre Dame (MR)

U. of Paderborn (II)

U. of Pennsylvania (CMC)

U. of Rostock (II)

U. of Tennessee (CMC, II)

U. of Texas Southwestern Medical Center (II)

U. of Vermont (II)

U. of Vienna Inst. für Materialphysik (II)

U. of Virginia/Dept. of Molec. Phys. (II)

$\mathrm{U}$. of Washington (PNC, II)

U. of Western Ontario (II)

U. of Wisconsin $(\mu$, II)

UOP (UNI*)

Warsaw U. Poland (II)

Washington U. $(\mu$, II)

Western Michigan U. (BESSRC, II)

X-Ray Analytics, Ltd. (SBC, II)

Yale U. (II)

*Both proprietary and nonproprietary Agreements have been signed.

p A Proprietary User Account has been established. 


\section{XFD Safety Report for FY 1998}

\section{Introduction}

An important element of XFD's mission is ensuring the safety of APS users, visitors, and APS/XFD personnel, and the protection of the environment. In keeping with Integrated Safety Management (ISM) Core Function 5, "provide feedback and continuous improvement," XFD is initiating periodic reporting of its safety-related activities and statistics, beginning with this summary for FY 1998.

\section{Safety Training for XFD Personnel}

\section{Determining Training Requirements}

The safety training requirements for most XFD personnel are determined by completing an ANL Job Hazard Questionnaire (JHQ). This document is used to identify the hazards employees encounter on the job. Approximately once each year, employees and their supervisors jointly review the JHQ to ensure that it reflects the employees' current assignments.

The data from completed JHQs are entered into ANL's Training Management System (TMS), a database that uses the JHQ responses and Division input to identify each individual's training requirements. The system produces a report, known as a Training Profile, that lists required and suggested training, dates when training was completed, and dates by which training/retraining must be completed.

XFD supervisors and managers have real-time access to the records of those they supervise thorough a password-protected Web-based window into the TMS. This enables them to verify that appropriate training is in place before assigning work. Employees also have access to their own records.

\section{Computer-Based and Web-Based Training}

The following courses are available as computer-based training (CBT) administered by the XFD ES\&H Office:

- ESH738, General Employee Radiation Training

- ESH700, Radiological Worker Training - Level 1

- ESH702, Radiological Worker Training - Level 2

- ESH707, Accelerator Worker Radiation Safety

- DIV832, Chemical Hazards Training - Agents that Damage the Skin, Mucous Membranes and Eyes

- DIV834, Chemical Hazards Training - Corrosives, Irritants, and Sensitizers

The following courses are available via the Web:

- ESH108/382, Building Safety Orientation

- ESH108/400/401, Building Safety Orientation 
- ESH223, Computer Protection

- ACIS/Tunnel Safety Courses

- Orientation for Chemical Management System Users (Nonmandatory)

\section{Radiological Worker Training}

All XFD personnel are required to complete ESH738, General Employee Radiation Training (GERT), and/or other courses from the following list as required by their work assignments:

- ESH700, Radiological Worker Training - Level 1

- ESH702, Radiological Worker Training - Level 2

- ESH707, Accelerator Worker Radiation Safety

- ESH709, Sealed Radioactive Source Custodian

- ESH713, Radiological Worker Training for X-ray Users

- APS457, APS Experimental Facilities Division X-Ray Laboratory Training

Most of these courses have both refresher and retraining requirements, which are met by attending courses with slightly different content and different course numbers.

\section{Training Completed by XFD Personnel}

To date, XFD personnel have attended more than 40 different safety courses and have collectively completed approximately 2,935 hours of training

\section{Training Compliance Progress}

XFD's compliance with training requirements, as calculated by TMS, has improved steadily throughout FY 1998 to $98.7 \%$ as of October 5, 1998.

\section{Safety Training for Users}

The responsibility for APS user safety training is shared by the APS and the CATs. This training falls into three broad categories:

"Core" training is required for all APS users and is administered by the APS User Office. As of July 30, 1998, 1,070 APS users have completed the core training program, which consists of the following elements:

APS User Orientation: This Web-based orientation, developed and updated as needed by XFD staff, is currently being delivered to newly arrived APS users via on-site computers; some preliminary experiments with remote delivery to users at their home institutions have been conducted. The general orientation course covers ANL and APS policies; general safety information such as site alarms, the use of 911, hazard communication, radiation safety, and experiment safety; and the basics of the beamline Personnel Safety System (PSS). Additional safety information is provided by the APS User Guide and the pocket- 
sized APS User Safety Guide, both prepared by XFD staff; these booklets are part of the user registration package and are also posted on the Web. All incoming users sign a statement confirming that they have read and understood both the APS User Guide and the User Orientation and will follow the guidelines given there.

General Employee Radiation Training (GERT): The GERT training is a general APS requirement consistent with DOE policy. APS users who do not have a current GERT card from another DOE facility must pass a computer-based GERT exam. They may prepare for the exam by taking a CBT course at the APS or via the Web, or by reading a hard-copy study guide, which is part of the user registration package.

Sector-specific training is also required for all APS users; it is administered by the CATs. To facilitate this training, XFD has provided a model checklist of topics to be covered; each CAT may modify the list as necessary. The training focuses on communicating specific information needed to implement the CAT's safety plan; examples include locations of utility shutoffs, chemical storage locations and practices, and beamline-specific operation of the PSS. The CAT signs off on the checklist and sends a copy to the APS User Office to verify that each new user who will be working under its auspices has completed this training. To date, the User Office has received signed-off "sector orientation records" for 797 APS users.

Task-specific training is the third element of APS user training. The CATs identify taskspecific training needs for their personnel and users in accordance with their CAT safety plans. Qualified CAT staff members may perform some of this training themselves; two examples are training users to operate the hoists in the experiment stations and orienting experienced machine tool users to the CAT's machine shop. Many other task-specific training needs are met, in whole or in part, through courses offered by ANL's ESH Division; the XFD ES\&H Coordinator's office handles user enrollments in these courses. XFD supports the course selection process both by oneon-one consultation with users and by maintaining an XFD-developed computer program that matches available courses and certifications to planned activities. XFD has also worked closely with the ESH Division's Training Section (ESH-TR) to tailor courses and course requirements to APS users' needs. A recent example is the condensed DOT HazMat Worker Training course described in Section 5 below. To date, APS users who are not employed by ANL have taken a total of 33 different optional and required ESH-TR courses and have collectively completed approximately 347 hours of formal ESH-TR training.

\section{Support Services Provided by the ANL ESH Division}

\section{Dosimetry}

At present, permanent dosimeters are assigned to approximately 182 XFD employees and 447 APS users. (Users who plan to spend only a short time at the APS are generally given temporary dosimeters.) In CY 1997, the total cumulative dosage received by all XFD personnel and APS users was $40 \mathrm{mrem}$. (The maximum permissible dose for a single individual classified as a "nonradiation worker" is $100 \mathrm{mrem} / \mathrm{yr}$.) For the period January-September 1998, the total cumulative dosage received by all XFD personnel and APS users was 20 mrem.

\section{Industrial Hygiene Surveys}

In March 1998, XFD personnel were surveyed for exposure to hazards associated with flame spray deposition of metals. Exposures to chromium and nickel were found to be well below 
permissible limits. Noise exposures continued to be high. Affected personnel already participate in a hearing conservation program and will continue to do so.

\section{Processes and Procedures}

\section{Transporting Small Quantities of Hazardous Materials}

The ANL Transportation Safety Board recently approved an XFD proposal to allow APS users to bring small quantities of certain nonradioactive hazardous materials (HazMats) directly to the APS in personal or rental vehicles, when packaged and transported in accordance with U.S. Department of Transportation (DOT) "Small Quantity Exception" regulations. The transport of these materials must be pre-approved by the CAT that is hosting the user's experiment, as part of the experiment safety review process described in Section 11 below. A condensed DOT HazMat Worker Training course has been provided to representatives of all the APS CATs to enable them to provide users of their beamlines with appropriate guidance and review. (A number of XFD staff members have also taken this course.) The training included an overview of other relevant DOT requirements and a brief review of DOT enforcement activities.

\section{Working with Radioactive Samples}

Users have begun doing experiments with radioactive samples at the APS. These samples present new requirements in terms of nuclear material accountability and radiation safety. XFD Operations personnel are working closely with Health Physics personnel in ANL's ESH Division to establish guidelines and develop procedures, and with the CATs to prepare and plan for these experiments. Certain categories of radioactive samples fall into the "accountable nuclear material" category, which carries with it additional DOE-imposed requirements for sample accountability and movement. A Web-based database is being developed to provide sample accountability by tracking the arrival of samples at the APS, sample movement between beamlines, and final disposition at the completion of the experiment. Preparations are underway to construct a dedicated area at the APS for receiving sample shipments and checking the integrity of the containment. Work is also ongoing to generate detailed radioactive-sample safety envelopes (see Section 11 below), which will classify samples and activity levels into well-defined categories with corresponding, predefined safety requirements. This information will greatly reduce the amount of time required by the CATs to plan experiments with radioactive samples.

\section{Chemical Tracking System}

In August 1998, XFD completed an inventorying activity that resulted in the bar-code labeling of Division-owned chemicals and the creation of records for these chemicals in the ANL Chemical Management System, or CMS. (Formerly, XFD had maintained records in an internally administered database.) Concurrently, the APS procurement system was enhanced to provide for the automatic entry of new chemicals into CMS at the time of purchase. Plans are now being made to bring APS users into the system.

\section{Laser Safety}

ANL's Laser Safety Officer (LSO) or Deputy LSO inspects all new Class 3b and Class 4 laser installations at the Laboratory before they are put into service, specifies required hazard controls, approves Standard Operating Procedures, and verifies that all laser operators (including APS users) are properly trained. The LSO and Deputy LSO are employees of the ANL ESH Division. Each CAT that uses a laser in one of the above classes has designated a Laser Custodian to ensure that the recommended controls, procedures, and training are implemented. The XFD ES\&H Coordinator has received the necessary training to serve as a Deputy LSO if the need arises. 


\section{XFD Operational Safety}

The World Wide Web is used extensively to convey operations and safety related information to the APS staff and users. All faults in XFD systems are recorded in the Web-based Trouble Reporting System. All planned work is entered into a Work Request System for approval and scheduling. Both of these systems provide a readily available database of information for tracking and trending faults and planning future work. Equipment tracking system information is provided through the Web for quick access to specific equipment failure and repair records. APS staff can use the Web to access the names and local phone numbers of designated contacts (XFD personnel for insertion device or front-end system faults, and CAT personnel for abnormal beamline situations). Emergency shutdown procedures for all systems are available on the Web to facilitate quick response in emergency situations. The configuration management records for beamline shielding are available to Operations personnel and can be easily updated by the Floor Coordinators as shielding status is changed. The Web also provides a record of current approved operating safety envelopes for all the beamlines.

\section{XFD Occupational Injury Experience}

Nature of Incident $\quad \begin{gathered}\text { No. of } \\ \text { Lost } \\ \text { Work- } \\ \text { days }\end{gathered} \quad \begin{gathered}\text { No. of } \\ \text { Restricted } \\ \text { Workdays }\end{gathered}$

1. While helping a custodial worker put trash into a dumpster, an employee slipped on a patch of ice and received a bruise.

2. A gasket failed after an employee changed a filter assembly in a high-pressure water system; the spraying water knocked him into a wall and caused a possible minor corneal abrasion.

3. An employee fabricated a makeshift tool, dropped it during use, and suffered a puncture wound in his palm while trying to catch it as it fell.
0

0

0

0

0

0
More thorough snow removal from the area was requested.

The supervisor prepared a written procedure for the task that described the hazards and the required hazard controls.

The task for which the tool had been fabricated was discontinued. 


\section{User Safety Experience}

Observations

\author{
Corrective Actions or Comments
}

1. CAT personnel observed a user working with $<0.5 \mathrm{~g}$ of uranyl acetate $(<280 \mathrm{nCi} / \mathrm{g})$ in a lab that was not a Radiological Control Area. The CAT stopped the work and ordered a survey; no contamination was found.

2. A shipping firm rejected two hydrogen sulfide cylinders (one empty, one holding about $20 \mathrm{~g}$ ) packaged by CAT personnel for off-site shipment. The shipment was accepted after the ANL Shipping Department provided assistance.

3. A CAT was using a class $3 \mathrm{~b}$ laser to align a class 4 laser without proper safeguards and prior authorization by the ANL Laser Safety Officer (LSO). The CAT's work on that beamline was stopped while XFD and the LSO worked with the CAT to ensure proper setup and operation of the Class 4 laser.

4. A user group removed chemical waste from the APS because of a misunderstanding of waste disposal procedures.

5. A user performed an experiment with samples that contained a small quantity $(<200 \mu \mathrm{g})$ of methylmercury. The samples were pre-encapsulated before arrival at the APS. The CAT had implemented proper hazard analysis, transport controls, personal protective equipment, signage, and standard operating procedures for the experiment.

6. The odor of thiophenol was detected outside a CAT's chemistry lab while a user was working with thiophenol in a hood in the lab. An industrial hygienist from the ESH Division investigated and determined that no risk was posed by the level of exposure that occurred.
The CAT has implemented more stringent requirements for supplying information via the Experiment Safety Approval form and instituted additional confirmations of that information.

The CAT was instructed in the proper procedures for shipping hazardous materials to/from ANL.

Class 2 lasers are now used for alignments as recommended. This CAT's laser installation has become a model for proper implementation of safeguards and standard operating procedures.

The CAT and experimenters received additional instruction about waste handling; CAT staff members have received the necessary training to properly accumulate and certify waste for pickup.

In an independent assessment of the CAT's safety program, its management of these samples was cited as a "noteworthy practice."

The industrial hygienist gave the experimenters additional instruction about working with this and similar compounds. No similar incidents have occurred since. 


\section{Safety Surveys and Results}

Between August 1997 and the time of this writing, XFD has conducted three self-assessments of its safety programs. In each case, a comprehensive survey of employees at all levels was used to gather the needed information. The scope and results of each self-assessment are summarized below.

XFD Integrated Safety Management Self-Assessment, August 1997: This self-assessment was designed to evaluate the degree to which XFD's activities incorporate the seven guiding principles and five core functions of ISM. Most XFD employees indicated that they are fully participating in, and seeing the benefits of, this integrated approach. The results also indicated that XFD's primary opportunity for safety improvement was in the area of supervisor participation in inspections. The Division followed up by having its managers implement the DuPont Safety Training Observation Program.

XFD Hazardous Materials Management Practices Self-Assessment, December 1997: This self-assessment looked at the degree to which XFD personnel understand and carry out assigned responsibilities and comply with ANL requirements for hazardous materials management. The results indicated a high level of awareness of and compliance with the requirements for procurement, storage, use, labeling, hazard communication, waste management, etc., and the application of good management practices. Some areas for improvement were identified, and corrective actions were implemented by XFD Group Leaders. These actions included initiating regular inspections of chemical labs and satellite waste accumulation areas and reminding Principal Investigators of the need to develop work plans and consult more closely with the Lab Safety Captains.

Gap Analysis for the Experimental Facilities Division, August 1998: This self-assessment was similar in scope to that of August 1997 (discussed above). The survey questions were designed to measure the degree to which XFD's practices met ISM criteria. Only minor gaps were identified. A corrective action plan is being formulated; it will include efforts to better communication existing policies, guidance, and inspection results to XFD personnel.

\section{Independent Assessments and Audits}

During FY 1998, XFD was the subject of independent oversight activities and audits covering the following areas:

Chemical Vulnerability, January 1998: This audit was conducted by a team of representatives from ANL's ESH/QA Oversight Group (EQO) and the DOE. The activity verified determinations previously reported by XFD in conjunction with its December 1997 Hazardous Materials Management Practices Assessment (see above). No findings resulted. The report recommended the integration of Divisional inventory databases into CMS, as XFD was already planning to do.

Radiation Protection Program, March 1998: ANL's ESH Division assessed the radiation protection programs implemented by both APS Divisions. The XFD program was found to be in compliance with 10 CFR 835 and Chapter 5 of the ANL Environment, Safety and Health Manual. 
The resulting report contained no safety-related findings or observations, and took note of five noteworthy procedures or processes.

ESH Training, April 1998: This review was conducted by representatives from DOE's Argonne Group as part of an ANL Program Review. No findings pertaining to XFD resulted; the report characterized XFD training records as "well maintained and complete" and acknowledged XFD's noteworthy practice of prohibiting certain activities unless required training had been verified.

Hoisting and Rigging Program, May-June 1998: This ANL-wide review was arranged and conducted by EQO with technical support provided by various ANL staff members. No findings or recommendations pertaining to XFD resulted.

Biosafety/Infection Control, July 1998: This review was conducted by representatives from DOE's Argonne Group as part of an ANL Program Review. No findings pertaining to XFD resulted. However, the DOE did recommend that XFD obtain evidence of training completed by APS users from other ANL Divisions. (XFD had already made arrangements for access to the TMS records of APS users who are not ANL employees.) XFD will inform the CATs that it may ask for evidence/documentation of training during experiment safety review oversight activities (see Section 12 below).

\section{User Experiment Safety}

The CATs have the primary responsibility for safety reviews of proposed experiments. The information needed to perform these reviews is obtained through the use of a standard APS Experiment Safety Approval Form (ESAF). In addition, some CATs have instituted more detailed experiment and safety questionnaires to be submitted with users' proposals. The forms are completed by the experimenter, who describes the materials and equipment to be used, the known hazards, and the ways in which these hazards will be mitigated. The CAT Director or designated safety coordinator reviews the information and makes recommendations as needed. The CATs may ask the XFD Experiment Safety Review Coordinator or the XFD ES\&H Coordinator to advise them on mitigating potential hazards, or to participate in the review process. When the safety review is completed, the CAT Director or designee lists any required hazard mitigation measures and signs off to indicate approval of the experiment. Before the experiment may begin, an individual designated by the CAT also verifies that all required safeguards are in place and signs the form. An APS Floor Coordinator posts the form at the beamline for the duration of the experiment. An XFD committee oversees the CAT experiment safety review process to ensure compliance with ANL safety requirements and to provide additional guidance on safety-related issues. This committee meets weekly to discuss ongoing and future experiments. The XFD Experiment Safety Review Coordinator serves as the liaison between the CAT Safety Coordinators and the APS.

The numbers of users and experiments at the APS, as reported on ESAFs, greatly increased during fiscal year 1998; 540 experiments were carried out in the course of 2,012 "user visits" involving 822 different individuals.

The APS is currently implementing two methods to assist the CATs with their experiment safety review process. The first, a Web-based system for the submission and approval of ESAFs, is currently in final beta testing and is expected to be implemented in early FY 1999. When a user submits an ESAF on-line, it will be transmitted to the appropriate CAT and, concurrently, to the XFD Experiment Safety Review Coordinator, who will be able to insert comments about any safety concerns the APS may have; those comments will be automatically transmitted to the CAT 
Safety Coordinator. The XFD Experiment Safety Review Coordinator will be notified when the CAT approves the experiment. When the users are ready to begin the experiment, the ESAF will be printed out for sign-off and posting as described above.

The Web-based system also includes safety guidance for the experimenters and the CATs. The guidance is based on the materials, hazards, and equipment that the experimenter checks off on the ESAF. The system will display either the requirements for working with a particular hazard or a link to other documentation on mitigating the hazard.

The second form of assistance that is now being implemented is a standard set of APS "experiment safety envelopes," which group proposed experiments on the basis of hazards. These envelopes have been developed in accordance with a graded approach that takes into account the severity of the hazards. Each envelope specifies a set of controls, including procedural and engineered controls, restrictions, shipping guidance, training requirements, and additional safety guidance references, that is sufficient to provide for the safe conduct of all individual experiments in that class. In addition, a set of Safety Guidelines for certain hazards is appended to the safety envelopes document. The guidelines provide more detailed information for working with individual hazards. When reviewing and approving an experiment, the CAT chooses the applicable envelope(s) and ensures that the specified controls are in place. The envelope(s) applicable to a given experiment are also listed on the ESAF that is posted at the beamline during the experiment.

The nine APS Experiment Safety Envelopes are:

1. APS Base Hazard Class

2. Cryogenic Hazards

3. High Temperatures

4. Class 3 and 4 Lasers

5. High Pressure Systems

6. Chemicals

7. Biosafety

8. Radioactivity

9. Other Hazards

The envelopes are currently being reviewed by the APS Research Directorate. The envelope descriptions will be posted on the Web and put into effect as soon as they are finalized. There will also be a link from the Web-based ESAF Safety Guide, which will list suggested envelopes for each experiment based on the hazards noted on the corresponding ESAF. The final determination of applicable safety envelopes is made by the CAT during the experiment safety review process.

\section{Safety Oversight}

\section{XFD Walkthroughs}

During FY 1998, XFD conducted a number of routine walkthrough inspections of spaces occupied by XFD employees and APS users. It also conducted one inspection in response to an Immediate Action Request from the ESH Division.

Ergonomics Walkthrough, September-October 1997: XFD personnel inspected office spaces as part of an ongoing self-assessment that focused on ergonomic issues. At XFD's request, an ergonomics specialist from the ESH Division participated. The observations resulted 
in substantial modifications to two XFD staff members' offices and minor modifications in other locations.

General Walkthrough, October 1997: XFD personnel conducted a general walkthrough of XFD- and CAT-occupied spaces. The walkthrough resulted in 25 observations that required follow-up; three of these remain open at the time of this writing.

General Walkthrough, February 1998: XFD personnel conducted another general walkthrough of XFD- and CAT-occupied spaces. The invited participants included a fire protection specialist and a safety specialist, both from the ESH Division. The walkthrough resulted in 13 observations that required follow-up; all have since been closed out.

General Walkthrough, July 1998: XFD personnel conducted another general walkthrough of XFD- and CAT-occupied spaces. The invited participants again included a fire protection specialist and a safety specialist from the ESH Division. The walkthrough resulted in several observations that required follow-up; all have since been closed out.

Immediate Action Request, July 1998: The ESH Division asked XFD to review the potential for localized oxygen-deficient or other hazardous atmospheres. In response, the XFD ES\&H Coordinator and User Technical Interface Group Leader conducted a walkthrough of XFDand user-occupied spaces. The only observation of note was a previously identified concern related to the use of liquid nitrogen. Owing to anticipated changes in the liquid nitrogen distribution system and the lack of any previous problems, further investigation will be deferred until the new system is put in place. However, the potential for catastrophic releases will be considered during the design of the new, centralized distribution system.

\section{Independent CAT Safety Assessments}

To take advantage of the CATs' growing experience in managing their own safety programs at the APS, XFD has initiated the formation of three Independent CAT Safety Assessment groups, within which the CATs conduct reciprocal assessments of each other's safety programs. XFD has provided a set of model assessment criteria. Each of the CATs currently in residence at the APS has named a representative to one of these groups, and the XFD Experiment Safety Review Coordinator is an ex officio member of all three groups. Each CAT is reviewed by the other CATs in its group at least annually, on a rotating basis. After a given CAT is reviewed, it receives a written report (which is copied to XFD) identifying action items and a schedule for completing these actions. The groups are also encouraged to make recommendations to the APS for enhanced safety support. Groups I and III have already started their assessment programs, and Group II will do so in early FY 1999.

The CATs' group affiliations, sector assignments (in parentheses), and assessment dates are shown below.

\begin{tabular}{|l|l|l|l|l|l|l|}
\hline \multicolumn{1}{|c|}{ Group I } & & & \multicolumn{1}{c|}{ Group II } & & \multicolumn{1}{c|}{ Group III } & \\
\hline BESSRC $(11,12)$ & $2 / 98$ & $1 / 99$ & IMM (8) & & Bio (sector 18) & \\
\hline CMC (9) & $6 / 98$ & $4 / 99$ & MHATT (7) & & CARS (sectors 13, 14, 15) & \\
\hline DND (5) & $9 / 97$ & $10 / 98$ & m (6) & & IMCA (sector 17) & \\
\hline SRI $(1,2,3)$ & $7 / 97$ & $7 / 98$ & PNC (20) & & MR (sector 10) & \\
\hline & & & UNI (33, 34) & & SBC (sector 19) & $6 / 98$ \\
\hline
\end{tabular}

The Independent Safety Assessment model criteria include (but are not limited to) the following steps: 
1. CAT safety overview presentation (CAT safety organization, communications, training, operations, safety planning, safety inspections, unique hazards, incident reports, follow-ups, lessons learned, etc.).

2. Questions by assessment team and inspection of safety-related records.

3. Walkthrough of CAT sector(s) and labs.

4. Executive session and close-out (report writing, action item identification, mitigation schedule, report to CAT of findings, recommendations to APS for safety improvements, and report distribution).

\section{XFD Safety Communication Activities}

\section{Safety Communication with XFD Employees}

Group Meetings: The XFD ES\&H Coordinator attends all meetings of the Experimental Floor Operations Group; since the XFD Floor Coordinators belong to this group, user safety issues are always a subject of discussion there. He also participates in most of the meetings of the other XFD groups, where he discusses general and group-specific safety issues.

Division Meetings: Safety issues are discussed at most XFD Division Meetings, which are held approximately twice a year. At the most recent Division Meeting, the Division Director reviewed the findings of XFD's August 1997 Integrated Safety Management Self-Assessment.

\section{Safety Communication with APS Users}

XFD has several forums for regular safety-related communication with APS users. At the quarterly APS Research Directorate Meetings, XFD management formally presents new safety policies and guidance to the CAT Directors as a group, and discusses any generic safety concerns that are best addressed by the CATs in a "top-down" manner. At the weekly "CAT Chats," XFD management discusses safety issues directly with the CAT staff members who manage and carry out the CATs' day-to-day activities on the experiment floor. In addition, safety information is made available to the entire user community both through the quarterly publication $C A T$ Communicator (which is mailed to about 1600 APS users) and via the APS Web site. 